\title{
Modelo assimétrico \\ com erros nas variáveis
}

\section{Nélida Susana Ozán}

\author{
Tese apresentada \\ ao \\ Instituto de Matemática e Estatística \\ da \\ Universidade de São Paulo \\ para \\ obtenção do grau \\ de \\ Doutor em Estatística
}

Área de Concentração: Estatística

Orientador: Prof. Dr. Heleno Bolfarine

-São Paulo, setembro de 2003- 


\section{Modelo assimérico}

\section{com erros nas variáveis}

Este exemplar corresponde à redação final da tese devidamente corrigida e defendida por Nélida Susana Ozán e aprovada pela comissão julgadora.

Comissão Julgadora:

- Prof. Dr. Heleno Bolfarine (Presidente) - IME-USP

- Profa. Dra. Marcia D’Elia Branco - IME-USP

- Prof. Dr. Filidor Edilfonso Vilca Labra - UNICAP

- Prof. Dr. Enrico Antônio Colosino - UFMG

- Prof. Dr. Reinaldo Boris Arellano Valle - PUC-Chile 
À minha mãe "La Pipo" 


\section{Agradecimentos}

Eu gostaria de agradecer a todos os que, direta ou indiretamente, contribuíram para a elaboração deste trabalho. Em especial,

- Ao Prof. Dr. Heleno Bolfarine, orientador e amigo, por sua dedicação e imensa paciência. Pela sua sempre presente disposição em atender e tirar dúvidas. Pela sua mão estendida nas horas difíceis e por tudo mais.

- Ao Prof. Dr. Reinaldo Boris Arellano-Valle, pelo aporte fundamental neste trabalho, por seu apoio, qualidade científica e sua amizade desde os tempos do mestrado, sempre grata.

- Ao Departamento de Estatística do IME-USP pelo excelente programa proporcionado e aos amigos que dele fazem parte.

- À CNPq pelo financiamento durante o programa de doutorado.

- Ao Departamento de Matemática da Faculdade de Engenharia da "Universidad Nacional de San Juan (Argentina)". Pela liberação para o programa, e aos amigos que lá deixei.

- Aos amigos e colegas, Michel e Felipe, por sua ajuda computacional de grande valor. A Dione, Suzy, Luciana, Adrilaine, Delhi, Carolina, Claudia, Juan, e especialmente: Silvina e Sílvia.

- A María, Eduardo, Luisa, Viviana e Daniel, pessoas inesquecíveis dos bons tempos no Brasil.

- Aos amigos que na distancia acompanharem com seu apoio e afeto: Yolanda, Claudia, Elisa e Laura, particularmente Emma e Maria Inés.

- À minha mãe pela força, a paciência e o amor.

Finalmente, agradeço a Deus, por ter possibilitado todos os agradecimentos acima. 
Capítulo 1

\section{Resumo}

Neste trabalho estudamos o modelo com erros nas variáveis considerando que os erros do modelo são distribuídas segundo uma classe particular de distribuições assimétricas.

O estudo é desenvolvido para o modelo estrutural, e são derivados resultados assintóticos que generalizam os obtidos na literatura sob a distribuição normal e distribuições elípticas. São feitas simulações e uma aplicação aos dados da Taxa de Mortalidade Infantil fornecidos pelo Sistema Único de Saúde (SUS) do Ministério da Saúde do Brasil.

\section{ABSTRACT}

The main object of this study in to consider measurement error models where the error distribution follows a particular class of skew distributions. We consider the structural measurement error model and obtain asymptotic results which generalize previous results obtained underthe normality assumption. Results of a simulation study is presented and an application to a data set form Sistema Único de Saúde do Ministério de Saúde. 


\section{Conteúdo}

Agradecimentos $\quad$ iv

1 Resumo v v

Lista de Tabelas viii

Lista de Figuras $\quad$ ix

1 Introdução 1

1.1 Formulação do Problema . . . . . . . . . . . . . . . . . . . . . . . 1

1.2 Definição dos Objetivos . . . . . . . . . . . . . . . . . . . 2

1.3 Organização do Trabalho . . . . . . . . . . . . . . . . . . . . 3

2 Distribuições Assimétricas 5

2.1 Introdução . . . . . . . . . . . . . . . . . . . . . . 5

2.2 Distribuições Assimétricas . . . . . . . . . . . . . . . . . 5

2.2 .1 Resultados . . . . . . . . . . . . . . . . . . . 8

2.3 Distribuição Normal Assimétrica . . . . . . . . . . . . . . . . . . . . . 14

3 Modelo Assimétrico com Erros nas Variáveis $\quad 16$

3.1 Introdução . . . . . . . . . . . . . . . . . . . . . . 16

3.2 Modelo Estrutural Assimétrico Linear Aditivo . . . . . . . . . . . . . . . . 16

3.2 .1 Especificação do Modelo . . . . . . . . . . . . . . . . . . . 16

3.2.2 Modelo Estrutural Assimétrico Linear Aditivo com variância constante 24

3.2 .3 Estimação . . . . . . . . . . . . . . . . . . . . . . . . 31

3.3 Modelo Estrutural Assimétrico Linear Multiplicativo . . . . . . . . . . . . 34

3.3 .1 Especificação do Modelo . . . . . . . . . . . . . . . . . . . . . . . . . 34

3.3.2 Modelo Estrutural Assimétrico Linear Multiplicativo com variância constante . . . . . . . . . . . . . . . . . 38

3.3 .3 Estimação . . . . . . . . . . . . . . . . . . . . . . . . . . . . 42

4 Modelo Normal Assimétrico com Erros nas Variáveis 49

4.1 Introdução . . . . . . . . . . . . . . . . . . . . . . . . . . 49 
4.2 Modelo Estrutural Normal Assimétrico . . . . . . . . . . . . . . . . . . . . . 49

4.2 .1 Especificação do Modelo . . . . . . . . . . . . . . . . . . . . . . . . 49

4.2 .2 A Função de Verossimilhança . . . . . . . . . . . . . . . . . . . . . . 51

4.2.3 Um resultado de invariância para Inferência Bayesiana . . . . . . . . . 58

4.3 Distribuição assintótica conjunta de $\left(\overline{\mathbf{Z}}, \mathbf{S}_{z}\right) \ldots \ldots \ldots$. . . . . . . 60

5 Inferência no Modelo Normal Assimétrico com Erros nas Variáveis $\quad 71$

5.1 Introdução . . . . . . . . . . . . . . . . . . . 71



5.2 .1 Variância $\sigma_{u}^{* 2}$ conhecida . . . . . . . . . . . . . . . . . 72

5.2 .2 Razão de variâncias $k_{x}^{*}$ conhecida . . . . . . . . . . . . . . . . 75

5.2 .3 Razão de variâncias $k_{e}^{*}$ conhecida . . . . . . . . . . . . . . . . . . 77

5.3 Estimação de Maxima Verossimilhança . . . . . . . . . . . . . . . . . . . . . 79

5.3 .1 Estudo de simulação . . . . . . . . . . . . . . . . . . . . . . 82

5.4 Aplicação . . . . . . . . . . . . . . . . . . . . . . . . . . . 84

5.4 Apresentação do problema . . . . . . . . . . . . . . . . . . 84

5.4 .2 Análise do problema . . . . . . . . . . . . . . 85

6 Conclusões $\quad 88$

6.1 Considerações Finais . . . . . . . . . . . . . . . . . . . . . . . . . . . . . . . . . . . . . . . . . . .

6.2 Propostas de trabalho futuro . . . . . . . . . . . . . 89

$\begin{array}{ll}\text { A Apêndice A } & 90\end{array}$

A.1 Método de Max-BFGS . . . . . . . . . . . . . . . . . . . . . . . . . . . 90

A.2 Programas de maximização . . . . . . . . . . . . . . . . . . . . . . 90

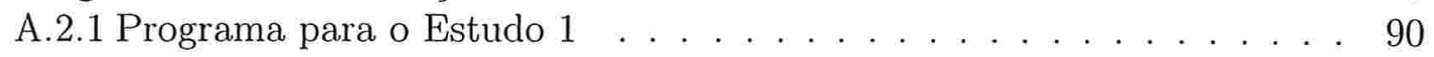

A.2.2 Programa para o Estudo 3 . . . . . . . . . . . . . . . . . . . . . 94

A.2.3 Programa para a Aplicação . . . . . . . . . . . . . . . . . . . . . . . . 99

$\begin{array}{ll}\text { Referências Bibliográficas } & 102\end{array}$

Ozán, N. S. 


\section{Lista de Tabelas}

3.1 Estimadores Modelo Assimétrico Aditivo . . . . . . . . . . . . . . . . . 47

3.2 Estimadores Modelo Assimétrico Multiplicativo, $\alpha=0 \ldots \ldots$. . . . . 47

3.3 Estimadores Modelo Assimétrico Multiplicativo, $\alpha$ conhecido . . . . . . . . 48

5.1 Estimativas de $\theta^{*} \ldots \ldots \ldots \ldots \ldots \ldots \ldots$

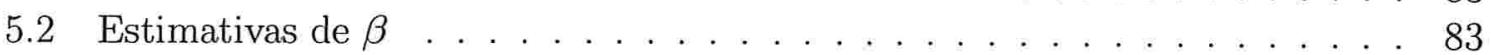

5.3 Estudo de simulação para $\beta \ldots \ldots \ldots \ldots \ldots \ldots \ldots$. . . . . . . . . . . . . . . . . . . . . . . . . . . .

5.4 Estatística Descritiva . . . . . . . . . . . . . . . . . 86



5.6 Taxa de Mortalidade Infantil - Proporção de Pobres . . . . . . . . . . . . 87 


\section{Lista de Figuras}

5.1 Gráfico Box-Plot . . . . . . . . . . . . . . . . . . . . 85

5.2 Gráfico de Assimetria . . . . . . . . . . . . . . . . . 85 


\section{Introdução}

\subsection{Formulação do Problema}

O desenvolvimento da modelagem de dados mediante o modelos de regressão linear é uma importante área na Estatística Aplicada. O modelo de regressão usual é dado por:

$$
Y=\alpha+\beta x+e
$$

com e o erro aleatório do modelo.

Em muitas situações as covariáveis sob estudo são medidas com erros devido, entre outras causas, à inexatidão dos instrumentos, fatores humanos, etc. Em muitas situações pode acontecer que o verdadeiro valor da covariável de interesse não possa ser medido ou observado diretamente, como por exemplo: dor, agressão, etc. Incluir este fato na modelagem, implica considerar um modelo para o erro de medição, que dá origem assim ao estudo do modelo com erros nas variáveis (Fuller, 1987; Caroll, Ruppert e Stefanski, 1995; Cheng e Van Ness, 1996).

No modelo linear com erros nas variáveis, temos interesse en estudar a relação linear entre a variável resposta $Y$ e a covariável $x$, que não é observada directamente e em seu lugar é observada uma quantidade que denotamos com $X$. Portanto a (1.1) adicionamos um modelo que explique a relação entre $X$ e $x$, que chamamos Modelo de Erro de Mediçấo e que pode ser aditivo: $X=x+u$, ou multiplicativo: $X=x . u$, onde $u$ é o erro aleatório do modelo.

Assim, segundo o modelo de erro de medição considerado temos um modelo com erro nas variáveis aditivo ou multiplicativo, de modo que,

$$
\begin{aligned}
& \text { Modelo Aditivo } \\
& \left\{\begin{array} { l l } 
{ Y = \alpha + \beta x + e . } & { \text { Modelo Multiplicativo } } \\
{ X = x + u }
\end{array} \quad \left\{\begin{array}{ll}
Y=\alpha+\beta x+e . \\
X=x \cdot u
\end{array}\right.\right.
\end{aligned}
$$

A covariável não observada $x$, pode ser considerada fixa ou aleatória, issto estabelece outra classificação para o modelo. Se $x$ é fixa o modelo se chama modelo funcional, e se $x$ é aleatório, o modelo é modelo estrutural.

Consideramos agora uma amostra de $n$ observações independentes, $\mathbf{Z}_{1}=\left(Y_{1}, X_{1}\right), \ldots$, 
$\mathrm{Z}_{n}=\left(Y_{n}, X_{n}\right)$, definimos o modelo ultraestrutural linear aditivo com erro nas variáveis (Dolby, 1976) como,

$$
\left\{\begin{array}{l}
Y_{i}=\alpha+\beta x_{i}+e_{i} \\
X_{i}=x_{i}+u_{i}
\end{array}\right.
$$

com

$$
e_{i} \stackrel{i i d}{\sim}\left(0, \sigma_{e i}^{2}\right), \quad u_{i} \stackrel{i i d}{\sim}\left(0, \sigma_{u i}^{2}\right) \quad \text { e } \quad x_{i} \stackrel{i i d}{\sim}\left(\mu_{x i}, \sigma_{x i}^{2}\right), \quad i=1, \ldots, n
$$

Portanto,

- se $\mu_{x i}=x_{i}$ e $\sigma_{x i}^{2}=0$, para $i=1, \ldots, n$, temos o modelo funcional,

- se $\mu_{x i}=\mu_{x}$, para $i=1, \ldots, n$, temos o modelo estrutural.

Notemos que considerando $\sigma_{e i}^{2}=\sigma_{e}^{2}, \sigma_{u i}^{2}=\sigma_{u}^{2}$ e $\sigma_{x i}^{2}=\sigma_{x}^{2}$, para todo $i=1, \ldots, n$, a distribuição assumida para o modelo tem variância constante.

O estudo do modelo com erros nas variáveis tem sido desenvolvido na literatura especializada, sob a hipóteses de normalidade em (1.3), Fuler (1987), Dolby (1976), Bolfarine e Cordani (1993), entre outros. Extensões considerando distribuições elípticas podem ser encontradas nos trabalhos de Arellano-Valle, Bolfarine e Vilca-Labra (1996) e ArellanoValle, Bolfarine e Gasco (2002).

O problema de identificabilidade do modelo infuencí a a estimação dos parâmetros no modelo estrutural, ver Fuller (1987), Glesser (1992) e Bolfarine e Cordani (1993). Um modelo é identificável se para dois valores diferentes do vector de parâmetros originam diferentes valores da função de densidade.

A solução proposta na literatura para o modelo elíptico não identificável, é considerar conhecida uma das variâncias $\sigma_{u}^{2}\left(\sigma_{e}^{2}\right)$, a razão de variâncias $\frac{\sigma_{e}^{2}}{\sigma_{u}^{2}}$ ou o coeficiente de confiabilidade $\frac{\sigma_{x}^{2}}{\sigma_{x}^{2}+\sigma_{u}^{2}}$. Considerações análogas são propostas para os modelos assimétricos com erros nas variáveis.

Considerar uma classe de distribuições mais gerais, que incluam as distribuições elípticas, tem sido o desafio deste trabalho. A extensão das distribuições elípticas é de grande interesse nas pesquisas dos últimos anos, como pode ser visto em Azzalini e Dalla-Valle (1996), Yuan e Bentler (1999a), Branco e Dey (2001 e Genton e Loperfido (2002). Portanto, para o modelo de regressão com erros nas variáveis, assumimos uma distribuição assimétrica em (1.3), obrendo a correspondente teoria assintótica para alguns estimadores consistentes.

\subsection{Definição dos Objetivos}

O objetivo geral deste trabalho é estudar modelos com erros nas variáveis assumiendo uma distribuição assimétrica em (1.3), definindo assim o modelo assimétrico com erros nas 
variáveis. Este tipo de distribuições se diferencia da distribuição normal, ou das elípticas, tanto pela curtose como pela assimetria. Como temos mencionado, existem várias formas de generalizar a distribuições elípticas. Além disso, é de nosso interesse analisar a inferência neste tipo de modelos.

Estabelecemos os seguintes objetivos específicos para nosso trabalho.

- Formular o modelo assimétrico com erros nas variáveis e o modelo normal assimétrico com erros nas variáveis.

- Obter a função de densidade para a variável observada $\mathrm{Z}=(Y, X)$, segundo o modelo normal assimétrico.

- Derivar a propriedades assintóticas para $\left(\overline{\mathbf{Z}}, \mathbf{S}_{Z}\right)$, sendo $\overline{\mathbf{Z}}=\frac{1}{n} \sum_{i=1}^{n} \mathbf{Z}_{i}$ e $\mathrm{S}_{Z}=\frac{1}{n} \sum_{i=1}^{n}\left(\mathrm{Z}_{i}-\overline{\mathbf{Z}}\right)\left(\mathbf{Z}_{i}-\overline{\mathbf{Z}}\right)^{T}$ para uma amostra de $n$ observações.

- Analisar o comportamento assintótico do estimador do parâmetro $\beta$.

- Estudar o método de máxima verossimilhança para estimar os parâmetros do modelo normal assimétrico com erro nas variáveis.

\subsection{Organização do Trabalho}

Este trabalho está organizado da seguinte forma.

No Capítulo 2, apresentamos as definições e propriedades das distribuições assimétricas consideradas neste trabalho. Primeiramente temos uma representação estocástica da distribuição assimétrica proposta por Yuan e Bentler (1999a). A seguir, a distribuição normal assimétrica independente, baseada na proposta de Azzalini (1985), derivando resultados a serem utilizados em capítulos seguintes.

No Capítulo 3, especificamos o modelo assimétrico linear aditivo com erros nas variáveis, obtendo resultados para determinar a variância assintótica do estimador do parâmetro $\beta$. O modelo linear multiplicativo também é analisado, fazendo as mesmas considerações que no caso aditivo.

No Capítulo 4, discutimos o modelo aditivo normal assimétrico, derivando a função de verossimilhança de $\mathrm{Z}$ e a distribuição assintótica de $\left(\overline{\mathrm{Z}}, \mathrm{S}_{Z}\right)$, tais resultados são utilizados na obtenção das propriedades assintóticas de estimadores do parâmetro $\beta$.

O problema de inferência no modelo normal assimétrico é apresentado no Capítulo 5.

Ozán, N. S.

IME/USP 
Analisamos condições para a consistência dos estimadores de $\beta$. O estimador de máxima verossimilhança é calculado por métodos numéricos, e apresentamos um estudo de simulação para analisar seu comportamento em termos de viés e erro quadrático médio. Completamos o trabalho com uma aplicação para dados reais.

Finalmente, no Capítulo 6, apresentamos as conclusões e discutimos algumas propostas de futuras pesquisas.

Os programas utilizados no estudo de simulação e aplicação são apresentados no Apêndice, como também a explicação dos métodos iterativos usados neste caso.

Ozán, N. S. 


\section{Distribuições Assimétricas}

\subsection{Introdução}

A extensão das distribuições simétricas para uma família de distribuições mas gerais, é abordada por diferentes autores nos últimos anos, especialmente a partir da publicação de Azzalini (1985). Azzalini apresenta uma classe de distribuições assimétricas, que define como distribuiçãoo skew normal, e para o caso multivariado ver Azzalini e Dalla Valle (1996). Extensões destas distribuições também são propostas por Branco e Dey (2000), Genton e Loperfido (2002), entre outros. Yuan e Bentler (1999) apresentam outro tipo de generalizações das distribuiçõoes elípticas, modificando a assimetria e a curtose, sim considerar a função de densidade da distribuição.

Neste capítulo, na primeira seção apresentamos a definição de distribuição assimétrica proposta por Yuan e Bentler, com considerações gerais e resultados a serem utilizados nos próximos capítulos. Segundo Fang, Kotz e Ng (1990), se Z é o vetor aleatório de dimensão $\mathrm{p}$, tal que $\mathrm{Z} \sim N_{p}(\mathbf{0}, \mathbf{I})$ e $v$ variável aleatória não negativa, então, $\mathrm{X}=v \Sigma^{1 / 2} \mathrm{Z}$ tem distribuição elíptica, onde $\Sigma$ é a matriz de variâncias covariâncias da vaariävel. A característica das distribuição elípticas são: simetria de $\mathbf{Z}$ e o fato de $v$ ser não negativa. A mudança de algumas destas condições permite a extensão a modelos mais gerais. Considerando esta idéia Yuan e Bentler (1999a), definem as chamadas distribuições não-elípticas, que incluem o caso elíptico, e que nós redefinimos com o nome de distribuições assimétricas.

Na seção 2.3, apresentamos a distribuição normal assimétrica independente, baseada na definição proposta por Azzalini (1985) e propriedades importantes para os próximos capítulos.

\subsection{Distribuições Assimétricas}

A definição que assumimos para as distribuições assimétricas esta baseada na construção proposta por Yuan e Bentler (1999a).

Definição 2.1. Distribuição Assimétrica.

Sejam 
- $\xi_{1}, \xi_{2}, \ldots, \xi_{m}$ variáveis aleatórias independentes com $E\left[\xi_{j}\right]=0, E\left[\xi_{j}^{2}\right]=1$, $E\left[\xi_{j}^{3}\right]=\zeta_{j}$ e $E\left[\xi_{j}^{4}\right]=\kappa_{j}, j=1, \ldots, m$ e $\boldsymbol{\xi}=\left(\xi_{1}, \xi_{2}, \ldots, \xi_{m}\right)^{T}$,

- $v$ variável aleatória independente de $\boldsymbol{\xi}$ com $E\left[v^{2}\right]=1, E\left[v^{3}\right]=\gamma$ e $E\left[v^{4}\right]=\delta, e$,

- $\mathbf{T}=\left(t_{i j}\right)$ matriz de dimensão $p \times m, m \geq p$, com posto de $\mathbf{T}$ igual a $p$, tal que $\mathbf{T T}^{T}=\Sigma$.

Então, a variável $\mathrm{X}$, de dimensão $p \times 1$, definida por:

$$
\mathrm{X}=v \mathbf{T} \xi,
$$

tem distribuição assimétrica com parâmetros de locação zero, de escala $\Sigma$, assimetria $\lambda$ e curtose $\boldsymbol{\kappa}$. Consideramos a seguinte notação:

$$
\mathrm{X} \sim S(0, \boldsymbol{\Sigma}, \boldsymbol{\lambda}, \boldsymbol{\kappa}) .
$$

O parâmetro de locação é de dimensão $p$ e é, neste caso, igual ao vetor de zeros pdimensional. Com relação ao parâmetro de escala, $\boldsymbol{\Sigma}$, matriz simétrica $p \times p$, temos que, $\boldsymbol{\Sigma}=\mathbf{T T}^{T}$, portanto existe ums relação entre a matriz $\mathbf{T}$ e a estrutura de covariâncias do modelo considerado.

No que diz respeito aos parâmetros de assimetria e curtose, vetores de dimensão $p$, que denotamos por $\boldsymbol{\lambda}=\left(\lambda\left(x_{1}\right), \lambda\left(x_{2}\right), \ldots, \lambda\left(x_{p}\right)\right)^{T}$ e $\kappa=\left(\kappa\left(x_{1}\right), \kappa\left(x_{2}\right), \ldots, \kappa\left(x_{p}\right)\right)^{T}$, respectivamente, onde, para $i=1, \ldots, p$, a assimetria e curtose marginal são,

$$
\lambda\left(x_{i}\right)=\gamma \sum_{j=1}^{m} \frac{t_{i j}^{3} \zeta_{j}}{\sigma_{i i}^{\frac{3}{2}}} \quad \text { e } \kappa\left(x_{i}\right)=\delta\left\{\sum_{j=1}^{m} \frac{t_{i j}^{4}\left(\kappa_{j}-3\right)}{\sigma_{i i}^{2}}+3\right\} .
$$

Considerando que $\lambda=f\left(\gamma, \zeta_{j}\right)$ e $\kappa=f\left(\delta, \kappa_{j}\right)$, para $j=1, \ldots, m$, podemos gerar diferentes distribuições mudando algumas destas condições. Portanto, se $E\left[\xi_{j}^{3}\right]=\zeta_{j} \neq 0$ modificamos a assimetria e se $E\left[\xi_{j}^{4}\right]=\kappa_{j} \neq 3$ mudamos a curtose da distribuição. Estas considerações originam as seguintes definições.

Definição 2.2. Distribuição Pseudo-elíptica.

Se X tem uma distribuição como em (2.1) e $\kappa_{j}=3$ para todo $j=1, \ldots, m$, então dizemos que $\mathrm{X}$ tem uma distribuição pseudo-elíptica.

$\mathrm{Na}$ distribuição pseudo-elíptica o coeficiente de curtose marginal é $\kappa\left(x_{i}\right)=3 \delta$ com assimetria arbitraria e é esta a diferencia com as distribuições elípticas. As distribuições elípticas são simétricas, onde só temos modificada a curtose, mas nas pseudo-elípticas o parâmetro de assimetria não precisa ser zero.

Definição 2.3. Distribuição Pseudo-Normal.

Se X tem uma distribuição como em (2.1), $\kappa_{j}=3$ para todo $j=1, \ldots, m$ e $\delta=1$, então dizemos que $\mathrm{X}$ tem uma distribuição pseudo-normal. 
No caso da distribuição pseudo-normal o coeficiente de curtose marginal é $\kappa\left(x_{i}\right)=3$ com assimetria arbitraria. Na distribuição normal o parâmetro de assimetria é zero, em quanto que na pseudo-normal não é necessariamente assim.

Exemplos destas distribuições são os seguintes.

- $\boldsymbol{\xi} \sim N_{m}(\mathbf{0}, \mathbf{I})$, com , $E\left[\xi_{j}^{3}\right]=\zeta_{j}=0$ e $E\left[\xi_{j}^{4}\right]=\kappa_{j}=3, j=1, \ldots, m$, e $v=1$, então, $\mathbf{X}$ tem distribuição normal.

- $\boldsymbol{\xi} \sim t_{m}(\nu)$ independente, com , $E\left[\xi_{j}^{3}\right]=\zeta_{j}=0$ e $E\left[\xi_{j}^{4}\right]=\kappa_{j} \neq 3, j=1, \ldots, m$, e $v=1$, então, $\mathrm{X}$ tem distribuiçãa $t$.

- Seja $\mathbf{Z} \sim N_{m}(\mathbf{0}, \mathbf{I})$, se consideramos $\xi_{1}=\left|Z_{1}\right|-E\left[\left|Z_{1}\right|\right]$, e $\xi_{j}=Z_{j}$ para $j=2, \ldots, p$, e $v=1$, então $\mathrm{X}$ tem distribuição normal assimétrica (Azzalini, 1985).

A variável $\mathrm{X}$ em $(2.1)$ é $\mathrm{X}=\left(x_{1}, x_{2}, \ldots, x_{p}\right)^{T}$, onde $x_{i}, i=1, \ldots, p$, pode ser escrita como,

$$
x_{i}=v \sum_{j=1}^{m} t_{i j} \xi_{j}
$$

Utilizando a expressão (2.4), derivamos os momentos da variável X. Portanto, temos que,

$$
\begin{aligned}
& E\left[x_{i}\right]=E\left[v \sum_{j=1}^{m} t_{i j} \xi_{j}\right]=0, \\
& E\left[x_{i}^{2}\right]=E\left[v^{2}\left(\sum_{j=1}^{m} t_{i j} \xi_{j}\right)^{2}\right]=\sum_{j=1}^{m} t_{i j}^{2}, \\
& E\left[x_{i}^{3}\right]=E\left[v^{3}\left(\sum_{j=1}^{m} t_{i j} \xi_{j}\right)^{3}\right]=\gamma \sum_{j=1}^{m} t_{i j}^{3} \zeta_{j}, \\
& E\left[x_{i}^{4}\right]=E\left[v^{4}\left(\sum_{j=1}^{m} t_{i j} \xi_{j}\right)^{4}\right]=\delta\left\{\sum_{j=1}^{m} t_{i j}^{4} \kappa_{j}+6 \sum_{s \neq r=1}^{m} t_{i s}^{2} t_{i r}^{2}\right\} .
\end{aligned}
$$
é,

Considerando uma seqüência $\mathrm{X}_{1}, \mathrm{X}_{2}, \ldots$ a expressão (2.1) para um elemento da seqüência

$$
\mathrm{X}_{k}=v_{k} \mathbf{T} \boldsymbol{\xi}_{k},
$$

onde $\xi_{k}=\left(\xi_{1 k}, \xi_{2 k}, \ldots, \xi_{m k}\right)^{T}$ e $v_{k}$ verificam as condições da Definição 2.1, para todo elemento $k$ da seqüência. Além disso, $\mathbf{T T}^{T}=\Sigma$, no caso de não considerar a matriz de covariância $\Sigma$ constante, temos que, $\mathbf{T}_{k} \mathbf{T}_{k}^{T}=\Sigma_{k}$. para todo $k$. 


\subsubsection{Resultados}

Consideramos as seguintes definições que serão usadas nos capítulos seguintes deste trabalho. Seja a matriz $\mathbf{A}=\left(a_{i j}\right)$ de dimensão $n \times m$. Denotamos por $\mathbf{A}_{j}$ a j-ésima coluna de $\mathbf{A}, j=1, \ldots, m$, definimos o vetor coluna de dimensão $n m, \operatorname{vec}(\mathbf{A})$, como $\operatorname{vec}(\mathbf{A})=\left(\mathbf{A}_{1}^{T}, \mathbf{A}_{2}^{T}, \ldots, \mathbf{A}_{m}^{T}\right)^{T}$. Se $\mathbf{A}$ é uma matriz simétrica de dimensão $p$, então vec $(\mathbf{A})$ tem elementos iguais, e portanto chamamos vech (A) ao vetor coluna de dimensão $\frac{p}{2}(p+1)$, com todos os elementos distintos (Magnus e Neudecker, 1988. Muirhead, 1982).

O resultado a seguir, apresenta a matriz de covariâncias para um vetor com distribuição assimétrica segundo a Definição 2.1.

Teorema 2.4. Se $\mathrm{X}$ é uma variável aleatória de dimensão $p$, com distribuição assimétrica segundo (2.1), então, $\Lambda=\operatorname{cov}\left\{v e c h\left(\mathrm{XX}^{T}\right)\right\}$ é dada por

$\Lambda=2 \delta \mathbf{D}_{p}^{+}(\boldsymbol{\Sigma} \otimes \boldsymbol{\Sigma}) \mathbf{D}_{p}^{+T}+(\delta-1) \operatorname{vech}(\boldsymbol{\Sigma}) \operatorname{vech}^{T}(\boldsymbol{\Sigma})+\delta \sum_{j=1}^{m}\left(\kappa_{j}-3\right) \operatorname{vech}\left(\mathbf{t}_{j} \mathbf{t}_{j}^{T}\right) \operatorname{vech}^{T}\left(\mathbf{t}_{j} \mathbf{t}_{j}^{T}\right)$

com $\mathbf{t}_{j}$ a j-ésima coluna de $\mathbf{T}$ e $\mathbf{D}_{p}^{+}$é a inversa generalizada de $\mathbf{D}_{p}$, sendo $\mathbf{D}_{p}$, uma matriz de dimensão $m \times p$, tal que vec $(\Sigma)=\mathbf{D}_{p}$ vech $(\boldsymbol{\Sigma})$ (Magnus e Neudecker, 1988, pag 49).

Demonstração. Seja $\mathbf{X}=v \mathbf{T} \boldsymbol{\xi} ;$ se $\mathbf{Y}=\mathbf{T} \boldsymbol{\xi}$, então, $\mathbf{X}=v \mathbf{Y}$.

$\operatorname{Logo}, \Lambda=\operatorname{cov}\left\{v e c h\left(\mathbf{X X}^{T}\right)\right\}=\operatorname{cov}\left\{v e c h\left(v \mathbf{Y} \mathbf{Y}^{T} v\right)\right\}=\operatorname{cov}\left\{v e c h\left(v^{2} \mathbf{Y} \mathbf{Y}^{T}\right)\right\}$, então,

$$
\Lambda=E\left[v^{4} \operatorname{vech}\left(\mathbf{Y} \mathbf{Y}^{T}\right) \operatorname{vech}\left(\mathbf{Y} \mathbf{Y}^{T}\right)\right]-E\left[v^{2} \operatorname{vech}\left(\mathbf{Y} \mathbf{Y}^{T}\right)\right] E^{T}\left[v^{2} \operatorname{vech}\left(\mathbf{Y} \mathbf{Y}^{T}\right)\right]
$$

e como $v$ e $\mathbf{Y}$ são independentes,

$$
\Lambda=E\left[v^{4}\right] E\left[\operatorname{vech}\left(\mathbf{Y} \mathbf{Y}^{T}\right) \operatorname{vech}^{T}\left(\mathbf{Y Y}^{T}\right)\right]-E\left[v^{2} \operatorname{vech}\left(\mathbf{Y} \mathbf{Y}^{T}\right)\right] E^{T}\left[v^{2} \operatorname{vech}\left(\mathbf{Y} \mathbf{Y}^{T}\right)\right]
$$

Por outro lado, considerando que $E(v \mathrm{Y})=0$, temos que,

$E\left[v^{2} \operatorname{vech}\left(\mathbf{Y} \mathbf{Y}^{T}\right)\right]=E\left[\operatorname{vech}\left(v^{2} \mathbf{Y} \mathbf{Y}^{T}\right)\right]=\operatorname{vech}\left[E\left(v^{2} \mathbf{Y} \mathbf{Y}^{T}\right)\right]=$

$=\operatorname{vech}\left[E\left(v^{2} \mathbf{Y} \mathbf{Y}^{T}\right)-E(v \mathbf{Y})\right]=\operatorname{vech}\left[E\left(\mathbf{X X}^{T}\right)-E(\mathbf{X}) E^{T}(\mathbf{X})\right]=$

$=\operatorname{vech}[\operatorname{cov}(\mathrm{X})]=\operatorname{vech}(\boldsymbol{\Sigma})$.

Logo em (2.7),

$$
\Lambda=E\left[v^{4}\right] E\left[\operatorname{vech}\left(\mathbf{Y} \mathbf{Y}^{T}\right) \operatorname{vech}^{T}\left(\mathbf{Y} \mathbf{Y}^{T}\right)\right]-\operatorname{vech}(\boldsymbol{\Sigma}) \operatorname{vech}^{T}(\Sigma),
$$

e como $E\left[v^{4}\right]=\delta$, temos que,

$$
\Lambda=\delta E\left[\operatorname{vech}\left(\mathrm{YY}^{T}\right) \operatorname{vech}^{T}\left(\mathrm{Y} \mathbf{Y}^{T}\right)\right]-\operatorname{vech}(\Sigma) \operatorname{vech}^{T}(\Sigma) .
$$

Para determinar $E\left[\operatorname{vech}\left(\mathbf{Y} \mathbf{Y}^{T}\right) \operatorname{vech}^{T}\left(\mathbf{Y} \mathbf{Y}^{T}\right)\right]$ usamos os seguintes lemas. 
Lema 2.5. Se $\xi_{1}, \xi_{2}, \ldots, \xi_{m}$ são variáveis aleatórias independentes com $E\left(\xi_{j}\right)=0$, $E\left(\xi_{j}^{2}\right)=1, E\left(\xi_{j}^{4}\right)=\kappa_{j}, j=1, \ldots, m, e \boldsymbol{\xi}=\left(\xi_{1}, \xi_{2}, \ldots, \xi_{m}\right)$, então,

$$
E\left[\operatorname{vec}\left(\boldsymbol{\xi} \xi^{T}\right) \operatorname{vec}{ }^{T}\left(\boldsymbol{\xi} \boldsymbol{\xi}^{T}\right)\right]=\mathbf{K}_{m}+\mathbf{I}_{m^{2}}+\operatorname{vec}\left(\mathbf{I}_{m}\right) \operatorname{vec}{ }^{T}\left(\mathbf{I}_{m}\right)+\sum_{i=1}^{m}\left(\kappa_{i}-3\right) \operatorname{vec}\left(\mathbf{E}_{i i}\right) v e c^{T}\left(\mathbf{E}_{i i}\right)
$$

onde, $\mathbf{K}_{m}$ é a matriz "comutação" (commutation, Magnus e Neckder, 1979), $\mathbf{I}_{m^{2}}=\mathbf{I}_{m} \otimes \mathbf{I}_{m}$ $e \mathbf{E}_{i j}=\mathbf{e}_{i} \mathbf{e}_{j}^{T} \operatorname{com} \mathbf{e}_{i}=(0, \dot{0}, \ldots, 1,0)$ (1 na posição $\left.i\right)$, idem para $\mathbf{e}_{j}$.

Demonstração. Como $\operatorname{vec}\left(\boldsymbol{\xi} \xi^{T}\right)=\boldsymbol{\xi} \otimes \boldsymbol{\xi}^{T}$, então, $v e c\left(\xi \xi^{T}\right) v e c^{T}\left(\xi \xi^{T}\right)=(\xi \otimes \xi)\left(\xi^{T} \otimes \xi^{T}\right)=\left(\xi \xi^{T}\right) \otimes\left(\xi \xi^{T}\right)=\sum_{i=1}^{m} \sum_{j=1}^{m} \mathbf{E}_{i j} \otimes \mathbf{B}_{i j}$, com $\mathbf{B}_{i j}=\xi_{i} \xi_{j}\left(\xi \xi^{T}\right)$, matriz de dimensão $m \times n$, e $\mathbf{E}_{i j}=\mathbf{e}_{i} \mathbf{e}_{j}^{T}$. Logo,

$$
E\left[\operatorname{vec}\left(\boldsymbol{\xi} \boldsymbol{\xi}^{T}\right) v e c^{T}\left(\boldsymbol{\xi} \xi^{T}\right)\right]=\sum_{i=1}^{m} \sum_{j=1}^{m} \mathbf{E}_{i j} \otimes E\left[\mathbf{B}_{i j}\right] .
$$

Determinemos $E\left[\mathbf{B}_{i j}\right]$.

- Se $i=j$ então $\mathbf{B}_{i i}=\xi_{i}^{2}\left(\xi \xi^{T}\right)$ e portanto $E\left[\mathbf{B}_{i i}\right]=\mathbf{I}_{m}+\left(\kappa_{i}-1\right) \mathbf{E}_{i i}$.

- Se $i \neq j$ então $\mathbf{B}_{i j}=\xi_{i} \xi_{j}\left(\xi \xi^{T}\right)$ e portanto $E\left[\mathbf{B}_{i j}\right]=\mathbf{E}_{i j}+\mathbf{E}_{j i}$.

Também podemos escrever,

$$
E\left[\mathbf{B}_{i j}\right]=\mathbf{E}_{i j}+\mathbf{E}_{j i}+\delta_{i j}\left\{\mathbf{I}_{m}+\frac{1}{2}\left(\kappa_{i}-3\right)\left(\mathbf{E}_{i j}+\mathbf{E}_{j i}\right)\right\},
$$

onde, $\delta_{i j}=1$ se $i=j$ e 0 caso contrário.

Logo,

$$
\begin{aligned}
& E\left[\operatorname{vec}\left(\boldsymbol{\xi} \xi^{T}\right) v e c^{T}\left(\xi \xi^{T}\right)\right]=\sum_{i=1}^{m} \sum_{j=1}^{m} \mathbf{E}_{i j} \otimes\left[\mathbf{E}_{i j}+\mathbf{E}_{j i}+\delta_{i j}\left\{\mathbf{I}_{m}+\frac{1}{2}\left(\kappa_{i}-3\right)\left(\mathbf{E}_{i j}+\mathbf{E}_{j i}\right)\right\}\right]= \\
= & \sum_{i=1}^{m} \sum_{j=1}^{m}\left[\mathbf{E}_{i j} \otimes\left(\mathbf{E}_{i j}+\mathbf{E}_{j i}\right)\right]+\sum_{i=1}^{m} \sum_{j=1}^{m}\left[\mathbf{E}_{i j} \otimes \delta_{i j}\left\{\mathbf{I}_{m}+\frac{1}{2}\left(\kappa_{i}-3\right)\left(\mathbf{E}_{i j}+\mathbf{E}_{j i}\right)\right\}\right]= \\
= & \sum_{i=1}^{m} \sum_{j=1}^{m}\left[\mathbf{E}_{i j} \otimes \mathbf{E}_{i j}+\mathbf{E}_{i j} \otimes \mathbf{E}_{j i}\right]+\sum_{i=1}^{m} \sum_{j=1}^{m}\left[\mathbf{E}_{i j} \otimes \delta_{i j} I_{m}\right]+\sum_{i=1}^{m} \sum_{j=1}^{m} \frac{1}{2}\left(\kappa_{i}-3\right) E_{i j} \otimes\left(\mathbf{E}_{i j}+\mathbf{E}_{j i}\right) .
\end{aligned}
$$

Por outro lado,

Ozán, N. S. 
- $\sum_{i=1}^{m} \sum_{j=1}^{m}\left[\mathbf{E}_{i j} \otimes \delta_{i j} I_{m}\right]=$

$=\sum_{i=1}^{m}\left\{\left[\mathbf{E}_{i 1} \otimes \delta_{i 1} I_{m}\right]+\ldots+\left[\mathbf{E}_{i i} \otimes \delta_{i i} \mathbf{I}_{m}\right]+\ldots+\left[\mathbf{E}_{i m} \otimes \delta_{i m} I_{m}\right]\right\}=$

$=\sum_{i=1}^{m} \mathbf{E}_{i i} \otimes \mathbf{I}_{m}=\mathbf{E}_{11} \otimes \mathbf{I}_{m}+\ldots+\mathbf{E}_{m m} \otimes \mathbf{I}_{m}=\mathbf{I}_{m} \otimes \mathbf{I}_{m}=\mathbf{I}_{m^{2}}$

- $\sum_{i=1}^{m} \sum_{j=1}^{m} \frac{1}{2}\left(\kappa_{i}-3\right) \mathbf{E}_{i j} \otimes\left(\mathbf{E}_{i j}+\mathbf{E}_{j i}\right)=$

$=\sum_{i=1}^{m} \frac{1}{2}\left(\kappa_{i}-3\right)\left[\mathbf{E}_{i 1} \otimes\left(\mathbf{E}_{i 1}+\mathbf{E}_{1 i}\right)+\ldots+\mathbf{E}_{i i} \otimes\left(\mathbf{E}_{i i}+\mathbf{E}_{i i}\right)+\ldots+\mathbf{E}_{i m} \otimes\left(\mathbf{E}_{i m}+\mathbf{E}_{m i}\right)\right]=$ $=\sum_{i=1}^{m} \frac{1}{2}\left(\kappa_{i}-3\right)\left[\mathbf{E}_{i i} \otimes 2 \mathbf{E}_{i i}\right]=\sum_{i=1}^{m}\left(\kappa_{i}-3\right)\left(\mathbf{E}_{i i} \otimes \mathbf{E}_{i i}\right)$.

Assim, temos que,

$$
\left.E\left[\operatorname{vec}\left(\boldsymbol{\xi} \boldsymbol{\xi}^{T}\right) \operatorname{vec}{ }^{T}\left(\boldsymbol{\xi} \boldsymbol{\xi}^{T}\right)\right]=\sum_{i=1}^{m} \sum_{j=1}^{m}\left[\mathbf{E}_{i j} \otimes \mathbf{E}_{i j}+\mathbf{E}_{i j} \otimes \mathbf{E}_{j i}\right]+I_{m^{2}}\right]+\sum_{i=1}^{m}\left(\kappa_{i}-3\right)\left(\mathbf{E}_{i i} \otimes \mathbf{E}_{i i}\right) .
$$

Além disso, por propriedades do produto Kronecker (Magnus e Neudecker, 1979), temos que,

- $\mathbf{E}_{i i} \otimes \mathbf{E}_{i i}=\operatorname{vec}\left(\mathbf{E}_{i i}\right) \operatorname{vec}{ }^{T}\left(\mathbf{E}_{i i}\right)$.

- $\sum_{i=1}^{m} \sum_{j=1}^{m}\left(\mathbf{E}_{i j} \otimes \mathbf{E}_{i j}\right)=\operatorname{vec}\left(\mathbf{I}_{m}\right) \operatorname{vec}^{T}\left(\mathbf{I}_{m}\right)$.

- $\sum_{i=1}^{m} \sum_{j=1}^{m}\left(\mathbf{E}_{i j} \otimes \mathbf{E}_{j i}\right)=\sum_{i=1}^{m} \sum_{j=1}^{m}\left(\mathbf{E}_{i j} \otimes \mathbf{E}_{i j}^{T}\right)=\mathbf{K}_{m}$.

Portanto,

$E\left[\operatorname{vec}\left(\boldsymbol{\xi} \xi^{T}\right) \operatorname{vec}{ }^{T}\left(\boldsymbol{\xi} \xi^{T}\right)\right]=\mathbf{K}_{m}+\mathbf{I}_{m^{2}}+\operatorname{vec}\left(\mathbf{I}_{m}\right) \operatorname{vec}{ }^{T}\left(\mathbf{I}_{m}\right)+\sum_{i=1}^{m}\left(\kappa_{i}-3\right) \operatorname{vec}\left(\mathbf{E}_{i i}\right) \operatorname{vec} c^{T}\left(\mathbf{E}_{i i}\right)$.

Observação 1. Como

$E\left[\operatorname{vec}\left(\boldsymbol{\xi} \xi^{T}\right)\right] E^{T}\left[\operatorname{vec}\left(\boldsymbol{\xi} \xi^{T}\right)=\operatorname{vec}\left[E\left(\boldsymbol{\xi} \xi^{T}\right)\right] v e c^{T}\left[E\left(\boldsymbol{\xi} \xi^{T}\right)\right]=\operatorname{vec}\left(\mathbf{I}_{m}\right) \operatorname{vec} c^{T}\left(\mathbf{I}_{m}\right)\right.$, usando o Lema 1 , temos,

$$
\operatorname{cov}\left[\operatorname{vec}\left(\xi \xi^{T}\right)\right]=\mathbf{K}_{m}+\mathbf{I}_{m^{2}}+\sum_{i=1}^{m}\left(\kappa_{i}-3\right) \operatorname{vec}\left(\mathbf{E}_{i i}\right) \operatorname{vec}{ }^{T}\left(\mathbf{E}_{i i}\right)
$$


Lema 2.6. Seja $\mathbf{Y}=\mathbf{T} \xi$, com $\mathbf{T T}^{T}=\mathbf{\Sigma}, \mathbf{T}=\left(\mathbf{t}_{1}, \mathbf{t}_{2}, \ldots, \mathbf{t}_{m}\right)$ onde $\mathbf{t}_{i}$ é o i-ésimo vetor coluna de $\mathbf{T}, i=1, \ldots, m$, e $\boldsymbol{\xi}$ o mesmo que no Lema 1. Então,

$$
\begin{aligned}
& E\left[\operatorname{vech}\left(\mathbf{Y} \mathbf{Y}^{T}\right) \operatorname{vech}^{T}\left(\mathbf{Y} \mathbf{Y}^{T}\right)\right]= \\
& =2 \mathbf{D}_{p}^{+}(\boldsymbol{\Sigma} \otimes \boldsymbol{\Sigma}) \mathbf{D}_{p}^{+^{T}}+\operatorname{vech}(\boldsymbol{\Sigma}) \operatorname{vech}^{T}(\boldsymbol{\Sigma})+\sum_{i=1}^{m}\left(\kappa_{i}-3\right) \operatorname{vech}\left(\mathbf{t}_{i} \mathbf{t}_{i}^{T}\right) \operatorname{vech}^{T}\left(\mathbf{t}_{i} \mathbf{t}_{i}^{T}\right)
\end{aligned}
$$

Demonstração. Como vech $\left(\mathbf{Y Y}^{T}\right)=\mathrm{D}_{p}^{+} \operatorname{vec}\left(\mathbf{Y} \mathbf{Y}^{T}\right)$ (Magnus e Neudecker, 1988), então vech $\left(\mathbf{Y} \mathbf{Y}^{T}\right)=\mathbf{D}_{p}^{+} \operatorname{vec}\left(\mathbf{T} \xi \mathbf{T}^{T} \boldsymbol{\xi}^{T}\right)=\mathbf{D}_{p}^{+}(\mathbf{T} \otimes \mathbf{T}) \operatorname{vec}\left(\xi \xi^{T}\right)$.

Assim, vech $\left(\mathbf{Y} \mathbf{Y}^{T}\right) \operatorname{vech}^{T}\left(\mathbf{Y} \mathbf{Y}^{T}\right)=\mathbf{D}_{p}^{+}(\mathbf{T} \otimes \mathbf{T}) \operatorname{vec}\left(\boldsymbol{\xi} \xi^{T}\right) v e c^{T}\left(\boldsymbol{\xi} \xi^{T}\right)\left(\mathbf{T}^{T} \otimes \mathbf{T}^{T}\right) \mathbf{D}_{p}^{+^{T}}$. Portanto,

$$
\begin{aligned}
E\left[\operatorname{vech}\left(\mathbf{Y} \mathbf{Y}^{T}\right) \operatorname{vech}^{T}\left(\mathbf{Y} \mathbf{Y}^{T}\right)\right] & =\mathbf{D}_{p}^{+}(\mathbf{T} \otimes \mathbf{T}) E\left[\operatorname{vec}\left(\xi \xi^{T}\right) v e c c^{T}\left(\xi \xi^{T}\right)\right]\left(\mathbf{T}^{T} \otimes \mathbf{T}^{T}\right) \mathbf{D}_{p}^{+^{T}} \\
& =\mathbf{D}_{p}^{+}(\mathbf{T} \otimes \mathbf{T})\left[\mathbf{K}_{m}+\mathbf{I}_{m^{2}}+\operatorname{vec}\left(\mathbf{I}_{m}\right) v e e^{T}\left(\mathbf{I}_{m}\right)+\right. \\
& \left.+\sum_{i=1}^{m}\left(\kappa_{i}-3\right) \operatorname{vec}\left(\mathbf{E}_{i i}\right) \operatorname{vec}{ }^{T}\left(\mathbf{E}_{i i}\right)\right]\left(\mathbf{T}^{T} \otimes \mathbf{T}^{T}\right) \mathbf{D}_{p}^{+^{T}} \\
& \left.=\mathbf{D}_{p}^{+} \mathbf{T} \otimes \mathbf{T}\right)\left(\mathbf{K}_{m}+\mathbf{I}_{m}\right)(\mathbf{T} \otimes \mathbf{T})^{T} \mathbf{D}_{p}^{+^{T}}+ \\
& +\mathbf{D}_{p}^{+}(\mathbf{T} \otimes \mathbf{T}) \operatorname{vec}\left(\mathbf{I}_{m}\right) v e c{ }^{T}\left(\mathbf{I}_{m}\right)(\mathbf{T} \otimes \mathbf{T})^{T} \mathbf{D}_{p}^{+^{T}}+ \\
& +\mathbf{D}_{p}^{+}(\mathbf{T} \otimes \mathbf{T})\left[\sum_{i=1}^{m}\left(\kappa_{i}-3\right) \operatorname{vec}\left(\mathbf{E}_{i i}\right) v e c^{T}\left(\mathbf{E}_{i i}\right)\right](\mathbf{T} \otimes \mathbf{T})^{T} \mathbf{D}_{p}^{+^{T}} .
\end{aligned}
$$

Analisando os termos da soma acima, temos que,

- $\mathbf{D}_{p}^{+}(\mathbf{T} \otimes \mathbf{T})\left(\mathbf{K}_{m}+\mathbf{I}_{m}\right)(\mathbf{T} \otimes \mathbf{T})^{T} \mathbf{D}_{p}^{+^{T}}=\mathbf{D}_{p}^{+}(\mathbf{T} \otimes \mathbf{T}) \mathbf{K}_{m}(\mathbf{T} \otimes \mathbf{T})^{T} \mathbf{D}_{p}^{+^{T}}+$ $+\mathbf{D}_{p}^{+}(\mathbf{T} \otimes \mathbf{T}) \mathbf{I}_{m}(\mathrm{~T} \otimes \mathbf{T})^{T} \mathbf{D}_{p}^{+^{T}}$.

Mas,

$\mathbf{D}_{p}^{+}(\mathbf{T} \otimes \mathbf{T}) \mathbf{K}_{m}(\mathbf{T} \otimes \mathbf{T})^{T} \mathbf{D}_{p}^{+^{T}}=\mathbf{D}_{p}^{+} \mathbf{K}_{m}(\mathbf{T} \otimes \mathbf{T})(\mathbf{T} \otimes \mathbf{T})^{T} \mathbf{D}_{p}^{+^{T}}$

$=\mathbf{D}_{p}^{+} \mathbf{K}_{m} \operatorname{vec}(\Sigma) \operatorname{vec}^{T}(\Sigma) \mathbf{D}_{p}^{+^{T}}=\mathbf{D}_{p}^{+} \operatorname{vec}(\Sigma) v e c^{T}(\Sigma) \mathbf{D}_{p}^{+^{T}}=\mathbf{D}_{p}^{+}(\Sigma \otimes \Sigma) \mathbf{D}_{p}^{+^{T}}$.

$\mathrm{D}_{p}^{+}(\mathrm{T} \otimes \mathrm{T}) \mathbf{I}_{m}(\mathrm{~T} \otimes \mathbf{T})^{T} \mathbf{D}_{p}^{+^{T}}=\mathrm{D}_{p}^{+}(\mathrm{T} \otimes \mathbf{T})\left(\mathrm{T}^{T} \otimes \mathrm{T}^{T}\right) \mathbf{D}_{p}^{+^{T}}$

$=\mathrm{D}_{p}^{+} \operatorname{vec}\left(\mathbf{T T}^{T}\right) \operatorname{vec}\left(\mathbf{T T}^{T}\right) \mathbf{D}_{p}^{+T}=\mathrm{D}_{p}^{+} \operatorname{vec}(\Sigma) \operatorname{vec}(\Sigma) \mathbf{D}_{p}^{+^{T}}=\mathrm{D}_{p}^{+}(\Sigma \otimes \Sigma) \mathbf{D}_{p}^{+^{T}}$.

Portanto,

$\mathrm{D}_{p}^{+}(\mathrm{T} \otimes \mathrm{T})\left(\mathrm{K}_{m}+\mathbf{I}_{m}\right)(\mathrm{T} \otimes \mathbf{T})^{T} \mathbf{D}_{p}^{+^{T}}=2 \mathbf{D}_{p}^{+}(\Sigma \otimes \Sigma) \mathbf{D}_{p}^{+^{T}}$

- $\mathrm{D}_{p}^{+}(\mathrm{T} \otimes \mathbf{T}) \operatorname{vec}\left(\mathbf{I}_{m}\right) \operatorname{vec}^{T}\left(\mathbf{I}_{m}\right)(\mathbf{T} \otimes \mathbf{T})^{T} \mathrm{D}_{p}^{+^{T}}=\mathrm{D}_{p}^{+} \operatorname{ves}(\Sigma) \operatorname{vec} c^{T}(\Sigma) D_{p}^{+^{T}}$ $=\operatorname{vech}(\Sigma) \operatorname{vech}^{T}(\Sigma)$. 


$$
\begin{aligned}
- & \mathbf{D}_{p}^{+}(\mathbf{T} \otimes \mathbf{T})\left[\sum_{i=1}^{m}\left(\kappa_{i}-3\right) \operatorname{vec}\left(\mathbf{E}_{i i}\right) v e c^{T}\left(\mathbf{E}_{i i}\right)\right](\mathbf{T} \otimes \mathbf{T})^{T} \mathbf{D}_{p}^{+^{T}}= \\
= & \sum_{i=1}^{m}\left(\kappa_{i}-3\right) \mathbf{D}_{p}^{+}(\mathbf{T} \otimes \mathbf{T}) \operatorname{vec}\left(\mathbf{E}_{i i}\right) v e c^{T}\left(\mathbf{E}_{i i}\right)(\mathbf{T} \otimes \mathbf{T})^{T} \mathbf{D}_{p}^{+^{T}}= \\
= & \sum_{i=1}^{m}\left(\kappa_{i}-3\right) \mathbf{D}_{p}^{+} \operatorname{vec}\left(\mathbf{t}_{i} \mathbf{t}_{i}^{T}\right) \operatorname{vec}^{T}\left(\mathbf{t}_{i} \mathbf{t}_{i}^{T}\right) \mathbf{D}_{p}^{+^{T}}=\sum_{i=1}^{m}\left(\kappa_{i}-3\right) \operatorname{vech}\left(\mathbf{t}_{i} \mathbf{t}_{i}^{\prime}\right) \operatorname{vech}^{T}\left(\mathbf{t}_{i} \mathbf{t}_{i}^{T}\right) .
\end{aligned}
$$

Logo,

$$
\begin{aligned}
& E\left[\operatorname{vech}\left(\mathbf{Y Y} \mathbf{Y}^{T}\right) \operatorname{vech}^{T}\left(\mathbf{Y} \mathbf{Y}^{T}\right)\right]= \\
& =2 \mathbf{D}_{p}^{+}(\boldsymbol{\Sigma} \otimes \mathbf{\Sigma}) \mathbf{D}_{p}^{+^{T}}+\operatorname{vech}(\boldsymbol{\Sigma}) \operatorname{vech}^{T}(\boldsymbol{\Sigma})+\sum_{i=1}^{m}\left(\kappa_{i}-3\right) \operatorname{vech}\left(\mathbf{t}_{i} \mathbf{t}_{i}^{\prime}\right) \operatorname{vech}^{T}\left(\mathbf{t}_{i} \mathbf{t}_{i}^{T}\right) .
\end{aligned}
$$

Portanto, para completar a prova do Teorema 2.4 consideramos os resultados do Lema 2.6 , assim temos que,

$$
\begin{aligned}
\Lambda= & \delta\left[2 \mathbf{D}_{p}^{+}(\boldsymbol{\Sigma} \otimes \boldsymbol{\Sigma}) \mathbf{D}_{p}^{+^{T}}+\operatorname{vech}(\boldsymbol{\Sigma}) \operatorname{vech}^{T}(\boldsymbol{\Sigma})+\sum_{i=1}^{m}\left(\kappa_{i}-3\right) \operatorname{vech}\left(\mathbf{t}_{i} \mathbf{t}_{i}^{T}\right) \operatorname{vech}^{T}\left(\mathbf{t}_{i} \mathbf{t}_{i}^{T}\right)\right]- \\
& -\operatorname{vech}(\boldsymbol{\Sigma}) \operatorname{vech}^{T}(\boldsymbol{\Sigma}),
\end{aligned}
$$

ou

$\Lambda=2 \delta \mathbf{D}_{p}^{+}(\Sigma \otimes \Sigma) \mathbf{D}_{p}^{+^{T}}+(\delta-1) \operatorname{vech}(\Sigma) \operatorname{vech}^{T}(\Sigma)+\delta \sum_{i=1}^{m}\left(\kappa_{i}-3\right) \operatorname{vech}\left(\mathbf{t}_{i} \mathbf{t}_{i}^{T}\right) \operatorname{vech}^{T}\left(\mathbf{t}_{i} \mathbf{t}_{i}^{T}\right)$, concluindo assim a prova do teorema.

Corolario 2.7. 1. Se $\kappa_{j}=3$ para todo $j=1, \ldots, m$, então

$$
\Lambda=\Lambda_{E}=2 \delta \mathbf{D}_{p}^{+}(\Sigma \otimes \Sigma) \mathbf{D}_{p}^{+T}+(\delta-1) \operatorname{vech}(\Sigma) \operatorname{vech}^{T}(\Sigma) .
$$

2. Se $\kappa_{j}=3$ para todo $j=1, \ldots, m$ e $\delta=1$, então

$$
\Lambda=\Lambda_{N}=2 \mathbf{D}_{p}^{+}(\Sigma \otimes \Sigma) \mathbf{D}_{p}^{+T} .
$$

É de nosso interesse derivar propriedades assintóticos no modelo assimétrico com erros nas variáveis. A seguir apresentamos resultados que serão usados nos próximos capítulos. Seja $\mathrm{X}_{1}, \mathrm{X}_{2}, \ldots, \mathrm{X}_{n}$ amostra aleatória, onde $\mathrm{X}_{i}$ tem distribuição assimétrica.

Considerando o vetor de médias $\overline{\mathrm{X}}=\frac{1}{n} \sum_{i=1}^{n} \mathbf{X}_{i}$, e a matriz de covariâncias amostrais $\left.\mathrm{S}_{X}=\frac{1}{n} \sum_{i=1}^{n}\left(\mathrm{X}_{i}-\overline{\mathrm{X}}\right)\left(\mathrm{X}_{i}-\overline{\mathrm{X}}\right)\right)^{T}$, assim temos os seguintes resultados (Yuan e Bentler, 1999).

Ozán, N. S. 
Teorema 2.8. Sejam uma variável $\mathrm{X}$ com distribuição assimétrica, $\mathrm{X}_{1}, \mathrm{X}_{2}, \ldots, \mathrm{X}_{n}$ amostra aleatória de $\mathrm{X}$ e $\mathbf{S}_{X}$ a matriz de covarâncias amostrais. Então

$$
\sqrt{n}\left(\operatorname{vech}\left(\mathbf{S}_{X}\right)-\operatorname{vech}(\boldsymbol{\Sigma})\right) \stackrel{d}{\rightarrow} N(\mathbf{0}, \mathbf{\Lambda})
$$

com $\Lambda$ e $\mathrm{D}_{p}^{+}$apresentados no Teorema 2.4.

Notemos que $\Lambda_{E}$, no Corolário 2.7, é a matriz de covariâncias assintótica de $\operatorname{vech}\left(\mathbf{S}_{X}\right)$ baseada numa distribuição elíptica com curtose $k=3 \delta$, e $\Lambda_{N}$ é a matriz de covariâncias assintótica de vech $\left(\mathbf{S}_{X}\right)$ baseada numa distribuição normal com média $\mu$ e matriz de covariância $\Sigma$.

Teorema 2.9. Seja $f=\left(f_{1}, f_{2}, \ldots, f_{s}\right)^{\prime}$ funçâo continua, definida sob $\mathcal{G}=\{$ vech $(\boldsymbol{\Sigma}): \boldsymbol{\Sigma}>$ $0\}$. Sendo $\mathrm{S}_{X}$ a matriz de covariâncias amostrais, baseada numa amostra da variável $\mathbf{X}$ com distribuição assimétrica, então,

$$
\sqrt{n}\left(f\left[\operatorname{vech}\left(\mathbf{S}_{X}\right)\right]-f[\operatorname{vech}(\boldsymbol{\Sigma})]\right) \stackrel{d}{\rightarrow} N(\mathbf{0}, \Omega),
$$

onde $\Omega=\Pi \Lambda \Pi^{T}, \Pi=\frac{\partial f}{\partial v e c h^{T}(\Sigma)}$ de posto s e $\Lambda$ a mesma do Teorema.2.4.

Corolario 2.10. 1. Se X tem distribuição pseudo elíptica, então,

$$
\Omega=\Omega_{E}=2 \delta \Pi \mathbf{D}_{p}^{+}(\Sigma \otimes \Sigma) \mathbf{D}_{p}^{+^{T}} \Pi^{T}+(\delta-1) \Pi v e c h(\Sigma) v_{e c h}^{T}(\Sigma) \Pi^{T} .
$$

2. Se X tem distribuição pseudo normal, então,

$$
\Omega=\Omega_{N}=2 \Pi D_{p}^{+}(\Sigma \otimes \Sigma) D_{p}^{+^{T}} \Pi^{T} .
$$

Corolario 2.11. Se, para $j=1, \ldots, m$, temos que $\Pi v e c h\left(\mathbf{t}_{j} \mathbf{t}_{j}^{T}\right)=0$, então,

$$
\sqrt{n}\left(f\left[\operatorname{vech}\left(\mathbf{S}_{X}\right)\right]-f[\operatorname{vech}(\Sigma)]\right) \stackrel{d}{\rightarrow} N\left(0, \delta \Omega_{N}\right) .
$$

Quando $\delta=1$ então

$$
\sqrt{n}(f[\operatorname{vech}(\mathbf{S})]-f[\operatorname{vech}(\Sigma)]) \stackrel{d}{\rightarrow} N\left(0, \Omega_{N}\right)
$$

Observação 2. Uma condição necessária e suficiente para $\Omega=\Omega_{N}$ em (2.11) e (2.12), é:

$$
\sum_{j=1}^{m}\left(\kappa_{j}-3\right) \Pi v e c h\left(\mathbf{t}_{j} \mathrm{t}_{j}^{T}\right) \operatorname{vech}^{T}\left(\mathrm{t}_{j} \mathbf{t}_{j}^{T}\right) \Pi^{T}=0 .
$$




\subsection{Distribuição Normal Assimétrica}

A definição de distribuição normal assimétrica foi originalmente definida em Azzalini (1985), para o caso univariado, que reproduzimos a seguir.

Definição 2.12. Distribuição Normal Assimétrica. (Azzalini, 1985).

Dizemos que a distribuição da variável aleatória $X$ é normal assimétrica com parâmetro de locação $\mu$, escala $\sigma^{2}$ e assimetria $\lambda$, se a função de densidade é dada por,

$$
f_{X}(x)=\frac{2}{\sigma^{2}} \phi\left(\frac{x-\mu}{\sigma}\right) \Phi\left(\lambda \frac{x-\mu}{\sigma}\right),
$$

onde, $\phi($.$) e \Phi($.$) são a função de densidade e distribuição acumulada, respectivamente,$ de uma distribuição $N(0,1)$.

Denotamos à distribuição normal assimétrica como:

$$
X \sim S N\left(\mu, \sigma^{2}, \lambda\right) .
$$

Para o caso particular quando $X \sim S N(0,1, \lambda)$ também usamos a notação, $X \sim S N(\lambda)$ e a função de densidade é $f_{X}(x)=2 \phi(x) \Phi(\lambda x)$.

A seguir apresentamos algumas propriedades de interesse para o desenvolvimento do presente trabalho (Azzalini, 1985).

1. Se $X \sim S N(0,1, \lambda)$, então,

$$
E[X]=\left(\frac{2}{\pi}\right)^{\frac{1}{2}} \delta, \text { e } \operatorname{Var}[X]=1-\frac{2}{\pi} \delta^{2},
$$

onde, $\delta=\frac{\lambda}{\left(1+\lambda^{2}\right)^{\frac{1}{2}}}$, de modo que, $\lambda=\frac{\delta}{\left(1-\delta^{2}\right)^{2}}$.

2. Se $X \sim S N(0,1, \lambda)$, então, $X^{2} \sim \chi_{1}^{2}$. É claro que, $E\left[X^{2}\right]=E\left[X^{4}\right]=1$.

3. Se $X \sim S N(0,1, \lambda)$, então,

$$
E\left[X^{2 k+1}\right]=\sqrt{\frac{2}{\pi}} \lambda\left(1+\lambda^{2}\right)^{-\left(k+\frac{1}{2}\right)} 2^{-k}(2 k+1) ! \sum_{j=0}^{k} \frac{j !(2 \lambda)^{2 j}}{(2 j+1) !(k-j) !} .
$$

Portanto, se

$$
\begin{aligned}
& k=0 \Rightarrow E[X]=\sqrt{\frac{2}{\pi}} \lambda\left(1+\lambda^{2}\right)^{-\frac{1}{2}}=\sqrt{\frac{2}{\pi}} \delta, \\
& k=1 \Rightarrow E\left[X^{3}\right]=\frac{4}{3} \sqrt{\frac{2}{\pi}} \frac{\lambda\left(3+2 \lambda^{2}\right)}{\left(1+\lambda^{2}\right)^{\frac{1}{2}}}=\frac{4}{3} \sqrt{\frac{2}{\pi}} \delta\left(3-\delta^{2}\right) .
\end{aligned}
$$


4. Sejam $Y_{0} \sim N(0,1)$ e $Y_{1} \sim N(0,1)$, independentes, então,

$$
X=\delta\left|Y_{0}\right|+\left(1-\delta^{2}\right)^{\frac{1}{2}} Y_{1}
$$

tem distribuição normal assimétrica de parâmetro $\lambda$, com $\delta$ definido em (2.16).

Definição 2.13. Distribuição Normal Assimétrica Independente.

Seja $\mathbf{X}=\left(X_{1}, \ldots, X_{p}\right)^{T}$ tal que $X_{i} \sim S N\left(0,1, \lambda_{i}\right)$, isto é, $f_{X_{i}}(x)=2 \phi\left(x_{i}\right) \Phi\left(\lambda_{i} x_{i}\right)$, $i=1, \ldots, k$. Então $\mathrm{X}$ tem distribuição normal assimétrica independente de ordem $p$, se

$$
f_{\mathrm{X}}(\mathrm{x})=2^{p} \phi_{p}(\mathrm{x}) \Phi_{p}(\mathbf{D}(\boldsymbol{\lambda}) \mathbf{x})
$$

onde, $\phi_{p}($.$) e \Phi_{p}($.$) são as funções de densidade e distribuição acumulada, respectivamente,$ de uma distribuiçãoo $N_{p}(\mathbf{0}, \mathbf{I})$, com $\mathbf{D}(\boldsymbol{\lambda})=\operatorname{diag}\left\{\lambda_{1}, \ldots, \lambda_{p}\right\}$ e $\boldsymbol{\lambda}=\left(\lambda_{1}, \ldots, \lambda_{p}\right)^{T}$.

Denotamos como

$$
\mathrm{X} \sim S N I_{p}(\mathbf{0}, \mathrm{I}, \boldsymbol{\lambda})
$$

à distribuição normal assimétrica independente de ordem $p$, com parâmetro de assimetria $\boldsymbol{\lambda}=\left(\lambda_{1}, \ldots, \lambda_{p}\right)^{T}$.

No Capítulo 4 usamos esta definição para derivar a função de verossimilhança do modelo normal assimétrico com erros nas variáveis. 


\section{Modelo Assimétrico com Erros nas Variáveis}

\subsection{Introdução}

Neste capítulo apresentamos o modelo estrutural assimétrico com erros nas variáveis, utilizando uma caracterização para o vector de errors do modelo segundo a definição 2.1. do Capítulo 2 (Yuan e Bentler, 1999), obtendo uma representação estocástica para os modelos lineares aditivo e multiplicativo, respectivamente. Enfatizamos que a definição de distribuição assimétrica assumida, neste caso, para caracterizar o modelo com erros nas variáveis, não considera uma função de densidade para $\mathbf{Z}=(Y, X)$.

Primeiramente, especificamos o modelo estrutural aditivo, obtendo a expressão para os momentos do vetor de erros.

Dada $\mathbf{Z}_{1}, \ldots, \mathbf{Z}_{n}$, amostra de $n$ observações independentes, derivamos a distribuição assintótica da média e a covariância amostral, isto é, $\overline{\mathbf{Z}}=\frac{1}{n} \sum_{i=1}^{n} \mathbf{Z}_{i}$ e $\mathbf{S}_{Z}=\frac{1}{n} \sum_{i=1}^{n}\left(\mathbf{Z}_{i}-\overline{\mathbf{Z}}\right)\left(\mathbf{Z}_{i}-\overline{\mathbf{Z}}\right)^{T}$.

Discutimos limitações quanto à obtenção dos estimadores pelo método de maxima verossimilhança, já que não temos uma expressão para a função de densidade de Z. É de grande interesse a estimação do parâmetro $\beta$, e para isso consideramos os estimadores usuais propostos na literatura (Fuller (1987), Arellano-Valle, Bolfarine e Vilca Labra (1996), entre outros) para analisar a variância assintótica dos mesmos.

Finalmente, analisamos o modelo estrutural multiplicativo, analogamente ao estudado no modelo aditivo.

\subsection{Modelo Estrutural Assimétrico Linear Aditivo}

\subsubsection{Especificação do Modelo}

O modelo estrutural linear aditivo com erro nas variáveis, pode ser definido como,

$$
\mathrm{Z}_{i}=\mathbf{a}+\mathrm{Br}_{i}, \quad i=1, \ldots, n
$$


onde $\mathbf{Z}_{i}=\left(Y_{i}, X_{i}\right)^{T}, \mathbf{a}=(\alpha, 0)^{T}, \mathbf{r}_{i}=\left(x_{i}, e_{i}, u_{i}\right)^{T} \mathrm{e}$

$$
\mathbf{B}=\left(\begin{array}{ccc}
\beta & 1 & 0 \\
1 & 0 & 1
\end{array}\right)
$$

com, $\left(Y_{i}, X_{i}\right)^{T}$ variáveis observadas, $e_{i} \sim\left(0, \sigma_{e_{i}}^{2}\right), u_{i} \sim\left(0, \sigma_{u_{i}}^{2}\right)$, os erros aleatórios do modelo, e $x_{i} \sim\left(\mu_{x}, \sigma_{x_{i}}^{2}\right), i=1, \ldots, n$, independentes.

Assumimos que,

- $\mathbf{r}_{i}-\boldsymbol{\eta}=\left(x_{i}-\mu_{x}, e_{i}, u_{i}\right)^{T}$ tem distribuição assimétrica (Definição 2.1), com média zero, matriz de covariância $\Psi_{i}=\operatorname{diag}\left\{\sigma_{x_{i}}^{2}, \sigma_{e_{i}}^{2}, \sigma_{u_{i}}^{2}\right\}$, assimetria $\boldsymbol{\lambda}_{i}$ e curtose $\boldsymbol{\kappa}_{i}$, onde $\eta=\left(\mu_{x}, 0,0\right)^{T}$, de modo que, $E\left[\mathbf{r}_{i}\right]=\eta$ e $\operatorname{Var}\left[\mathbf{r}_{i}\right]=\Psi_{i}$, para $i=1, \ldots, n$.

Definindo $\mathbf{r}_{i}^{*}=\mathbf{r}_{i}-\boldsymbol{\eta}$, por (2.1) temos que,

$$
\mathrm{r}_{i}^{*}=v_{i} \mathbf{A}_{i} \boldsymbol{\xi}_{i}, \quad i=1, \ldots, n,
$$

onde,

1. $\boldsymbol{\xi}_{i}=\left(\xi_{1 i}, \ldots, \xi_{m i}\right)^{T}$, tal que $\xi_{1 i}, \ldots, \xi_{m i}$ são variáveis aleatórias independentes com $E\left[\xi_{j i}\right]=0, E\left[\xi_{j i}^{2}\right]=1, E\left[\xi_{j i}^{3}\right]=\zeta_{j}$ e $E\left[\xi_{j i}^{4}\right]=\kappa_{j}, j=1, \ldots, m$ e $i=1, \ldots, n$.

2. $v_{i}$ variável aleatória independente de $\boldsymbol{\xi}_{i} \operatorname{com} E\left[v_{i}^{2}\right]=1, E\left[v_{i}^{3}\right]=\gamma$ e $E\left[v_{i}^{4}\right]=\delta$,

3. $\mathbf{A}_{i}=\left(a_{i_{j}}\right)$, matriz de dimensão $3 \times m$, com posto igual a 3 , tal que $\mathbf{A}_{i} \mathbf{A}_{i}^{T}=\mathbf{\Psi}_{i}$, $i=1, \ldots, n, l=1,2,3$ e $j=1, \ldots, m$.

Usando a expressão (2.3), temos que a assimetria e curtose marginais, são, respectivamente dadas por,

$$
\begin{gathered}
\lambda_{1 i}=\lambda_{i}\left(x_{i}-\mu_{x}\right)=\gamma \frac{\sum_{j=1}^{m} a_{i_{1 j}}^{3} \zeta_{j}}{\left(\sum_{j=1}^{m} a_{i_{1 j}}^{2}\right)^{\frac{3}{2}}}, \quad \kappa_{1 i}=\kappa_{i}\left(x_{i}-\mu_{x}\right)=\delta\left\{\sum_{j=1}^{m} \frac{a_{i_{1 j}}^{4}\left(\kappa_{j}-3\right)}{\sigma_{x_{i}}^{2}}+3\right\}, \\
\lambda_{2 i}=\lambda_{i}\left(e_{i}\right)=\gamma \frac{\sum_{j=1}^{m} a_{i_{2 j}}^{3} \zeta_{j}}{\left.\sum_{j=1}^{m} a_{i_{2 j}}^{2}\right)^{\frac{3}{2}}}, \quad \kappa_{2 i}=\kappa_{i}\left(e_{i}\right)=\delta\left\{\sum_{j=1}^{m} \frac{a_{i_{2 j}}^{4}\left(\kappa_{j}-3\right)}{\sigma_{e_{i}}^{2}}+3\right\}, \\
\lambda_{3 i}^{m}=\lambda_{i}\left(u_{i}\right)=\gamma \frac{a_{i_{3 j}}^{3} \zeta_{j}}{\left(\sum_{j=1}^{m} a_{i_{3 j}}^{2}\right)^{\frac{3}{2}}}, \quad \kappa_{3 i}=\kappa_{i}\left(u_{i}\right)=\delta\left\{\sum_{j=1}^{m} \frac{a_{i_{3 j}}^{4}\left(\kappa_{j}-3\right)}{\sigma_{u_{i}}^{2}}+3\right\} .
\end{gathered}
$$

Ozán, N. S. 
Utilizando a notação (2.2), escrevemos que,

$$
\mathbf{r}^{*}=\mathbf{r}_{i}-\boldsymbol{\eta}=\left(x_{i}-\mu_{x}, e_{i}, u_{i}\right)^{T} \sim S\left(0, \Psi_{i}, \lambda_{i}, \kappa_{i}\right),
$$

$\operatorname{com} \lambda_{i}=\left(\lambda_{1 i}, \lambda_{2 i}, \lambda_{3 i}\right)^{T}$ e $\kappa_{i}=\left(\kappa_{1 i}, \kappa_{2 i}, \kappa_{3 i}\right)^{T}$ para $i=1, \ldots, n$.

A matriz $\mathbf{A}_{i}$, que esta relacionada com a estrutura de covariâncias da variável $\mathbf{r}_{i}^{*}$, verifica a seguinte propriedade.

Propriedade 1. Seja $\mathbf{A}_{i}$ como em 3 da expressão (3.2), e $\boldsymbol{\Psi}_{i}=\operatorname{diag}\left\{\sigma_{x_{i}}^{2}, \sigma_{e_{i}}^{2}, \sigma_{u_{i}}^{2}\right\}$, $i=1, \ldots, n$, então os elementos de $\mathbf{A}_{i}$ verificam a seguinte igualdade:

$$
\sum_{j=1}^{m} a_{i_{1 j}} a_{i_{2 j}}=\sum_{j=1}^{m} a_{i_{2 j}} a_{i_{3 j}}=\sum_{j=1}^{m} a_{i_{1 j}} a_{i_{3 j}}=0 .
$$

Além disso temos que,

$$
\sum_{j=1}^{m} a_{i_{1 j}}^{2}=\sigma_{x_{i}}^{2}, \quad \sum_{j=1}^{m} a_{i_{2 j}}^{2}=\sigma_{e_{i}}^{2}, \quad \sum_{j=1}^{m} a_{i_{3 j}}^{2}=\sigma_{u_{i}}^{2} .
$$

Demonstração. Se $\mathbf{A}_{i}=\left(a_{i_{l j}}\right), i=1, \ldots, n, l=1,2,3$ e $j=1, \ldots, m$, então,

$$
\mathbf{A}_{i}=\left(\begin{array}{cccc}
a_{i_{11}} & a_{i_{12}} & \ldots & a_{i_{1 m}} \\
a_{i_{21}} & a_{i_{22}} & \ldots & a_{i_{2 m}} \\
a_{i_{31}} & a_{i_{32}} & \ldots & a_{i_{3 m}}
\end{array}\right) \text {. }
$$

Assim, para $i=1, \ldots, n$,

$$
\mathbf{A}_{i} \mathbf{A}_{i}^{T}=\left(\begin{array}{ccc}
\sum_{j=1}^{m} a_{i_{1 j}}^{2} & \sum_{j=1}^{m} a_{i_{1 j}} a_{i_{2 j}} & \sum_{j=1}^{m} a_{i_{1 j}} a_{i_{3 j}} \\
\cdots & \sum_{j=1}^{m} a_{i_{2 j}}^{2} & \sum_{j=1}^{m} a_{i_{2 j}} a_{i_{3 j}} \\
\ldots & \cdots & \sum_{j=1}^{m} a_{i_{3 j}}^{2}
\end{array}\right)
$$

e como, o especificado em (3.2), $\mathrm{A}_{i} \mathrm{~A}_{i}^{T}=\Psi_{i}$, temos que,

$$
\left(\begin{array}{ccc}
\sum_{j=1}^{m} a_{i_{1 j}}^{2} & \sum_{j=1}^{m} a_{i_{1 j}} a_{i_{2 j}} & \sum_{j=1}^{m} a_{i_{1 j}} a_{i_{3 j}} \\
\ldots & \sum_{j=1}^{m} a_{i_{2 j}}^{2} & \sum_{j=1}^{m} a_{i_{2 j}} a_{i_{3 j}} \\
\ldots & \ldots & \sum_{j=1}^{m} a_{i_{3 j}}^{2}
\end{array}\right)=\left(\begin{array}{ccc}
\sigma_{x_{i}}^{2} & 0 & 0 \\
0 & \sigma_{e_{i}}^{2} & 0 \\
0 & 0 & \sigma_{u_{i}}^{2}
\end{array}\right) .
$$

Comparando os elementos da igualdade acima fica provada a propriedade.

Ozán, N. S. 
Analisando os momentos para $\mathbf{r}_{i}^{*}$ temos os seguintes resultados.

Proposição 3.1. Seja $\mathbf{r}_{i}^{*}$ como em (3.2), $i=1, \ldots, n$, então,

$$
\begin{aligned}
E\left[\mathbf{r}_{i}^{*}\right] & =0, \\
E\left[\mathbf{r}_{i}^{*} \mathbf{r}_{i}^{* T}\right] & =\operatorname{diag}\left\{\sum_{j=1}^{m} a_{i_{1 j}}^{2}, \sum_{j=1}^{m} a_{i_{2 j}}^{2}, \sum_{j=1}^{m} a_{i_{3 j}}^{2}\right\}=\Psi_{i}, e \\
E\left[\mathbf{r}_{i}^{*} \mathbf{r}_{i}^{* T} \mathbf{r}_{i}^{*}\right] & =\gamma\left(\sum_{j=1}^{m} a_{i_{1 j}}^{3} \zeta_{j}, \sum_{j=1}^{m} a_{i_{2 j}}^{3} \zeta_{j}, \sum_{j=1}^{m} a_{i_{3 j}}^{3} \zeta_{j}\right)^{T} .
\end{aligned}
$$

Demonstração. Sendo $\mathbf{r}_{i}^{*}=\mathbf{r}_{i}-\eta=v_{i} \mathbf{A}_{i} \boldsymbol{\xi}_{i}, i=1, \ldots, n$, então,

$$
\mathbf{r}_{i}^{*}=\left(\begin{array}{c}
x_{i}-\mu_{x} \\
e_{i} \\
u_{i}
\end{array}\right)=v_{i}\left(\begin{array}{c}
\sum_{j=1}^{m} a_{i_{1 j}} \xi_{j i} \\
\sum_{j=1}^{m} a_{i_{2 j}} \xi_{j i} \\
\sum_{j=1}^{m} a_{i_{3 j}} \xi_{j i}
\end{array}\right) .
$$

Portanto,

$$
x_{i}-\mu_{x}=v_{i} \sum_{j=1}^{m} a_{i_{1 j}} \xi_{j i}, \quad e_{i}=v_{i} \sum_{j=1}^{m} a_{i_{2 j}} \xi_{j i}, \quad u_{i}=v_{i} \sum_{j=1}^{m} a_{i_{3 j}} \xi_{j i} .
$$

Usando as expressões (2.5) para os momentos da distribuição assimétrica, temos que,

$$
\begin{array}{lll}
E\left(x_{i}-\mu_{x}\right)=0, & E\left(e_{i}\right)=0, & E\left(u_{i}\right)=0, \\
E\left[\left(x_{i}-\mu_{x}\right)^{2}\right]=\sum_{j=1}^{m} a_{i_{1 j}}^{2}, & E\left(e_{i}^{2}\right)=\sum_{j=1}^{m} a_{i_{2 j}}^{2}, & E\left(u_{i}^{2}\right)=\sum_{j=1}^{m} a_{i_{3 j}}^{2}, \\
E\left[\left(x_{i}-\mu_{x}\right)^{3}\right]=\gamma \sum_{j=1}^{m} a_{i_{1 j}}^{3} \zeta_{j}, & E\left(e_{i}^{3}\right)=\gamma \sum_{j=1}^{m} a_{i_{2 j}}^{3} \zeta_{j}, & E\left(u_{i}^{3}\right)=\gamma \sum_{j=1}^{m} a_{i_{3 j}}^{3} \zeta_{j},
\end{array}
$$

e,

$$
\begin{aligned}
& E\left[\left(x_{i}-\mu_{x}\right)^{4}\right]=\delta\left\{\sum_{j=1}^{m} a_{i_{i j}}^{4} \kappa_{j}+6 \sum_{s \neq r=1}^{m} a_{i_{1 s}}^{2} a_{i_{1 r}}^{2}\right\}, E\left(e_{i}^{4}\right)=\delta\left\{\sum_{j=1}^{m} a_{i_{2 j}}^{4} \kappa_{j}+6 \sum_{s \neq r=1}^{m} a_{i_{2 s}}^{2} a_{i_{2 r}}^{2}\right\}, \\
& E\left(u_{i}^{4}\right)=\delta\left\{\sum_{j=1}^{m} a_{i_{2 j}}^{4} \kappa_{j}+6 \sum_{s \neq r=1}^{m} a_{i_{2 s}}^{2} a_{i_{2 r}}^{2}\right\} .
\end{aligned}
$$

Ozán, N. S. 
Usando (3.5), temos que, $E\left[\mathrm{r}_{i}^{*}\right]=0$.

Por outro lado,

$$
\begin{aligned}
& \mathrm{r}_{i}^{*} \mathrm{r}_{i}^{* T}=v_{i}^{2}\left(\begin{array}{l}
\sum_{\substack{j=1 \\
m}}^{m} a_{i_{1 j}} \xi_{j i} \\
\sum_{\substack{m=1 \\
m}}^{m} a_{i_{2 j}} \xi_{j i} \\
\sum_{i_{3 j}} \xi_{j i}
\end{array}\right)\left(\sum_{j=1}^{m} a_{i_{1 j}} \xi_{j i} \sum_{j=1}^{m} a_{i_{2 j}} \xi_{j i} \sum_{j=1}^{m} a_{i_{3 j}} \xi_{j i}\right) \\
& =v_{i}^{2}\left(\begin{array}{ccc}
{\left[\sum_{j=1}^{m} a_{i_{1 j}} \xi_{j i}\right]^{2}} & \sum_{j=1}^{m} a_{i_{1 j}} \xi_{j i} \sum_{j=1}^{m} a_{i_{2 j}} \xi_{j i} & \sum_{j=1}^{m} a_{i_{1 j}} \xi_{j i} \sum_{j=1}^{m} a_{i_{3 j}} \xi_{j i} \\
\ldots & {\left[\sum_{j=1}^{m} a_{i_{2 j}} \xi_{j i}\right]^{2}} & \sum_{j=1}^{m} a_{i_{2 j}} \xi_{j i} \sum_{j=1}^{m} a_{i_{3 j}} \xi_{j i} \\
\ldots & \ldots & {\left[\sum_{j=1}^{m} a_{i_{3 j}} \xi_{j i}\right]^{2}}
\end{array}\right) .
\end{aligned}
$$

Para simplificar os cálculos, consideramos os elementos da matriz $\mathbf{r}_{i}^{*} \mathbf{r}_{i}^{* T}$ em (3.7), como $\mathbf{r}_{i}^{*} \mathbf{r}_{i}^{* T}=v_{i}^{2}\left(b_{l j}\right)$, com $l, j=1,2,3$ para $i=1, \ldots, n$. Portanto, com $l, j=1,2,3, i=1, \ldots, n$, temos que, $E\left[\mathbf{r}_{i}^{*} \mathbf{r}_{i}^{* T}\right]=E\left[v_{i}^{2}\left(b_{l j}\right)\right]=\left(E\left[b_{l j}\right]\right)$.

Assim,

$$
\begin{aligned}
E\left[b_{11}\right] & =E\left[\left(\sum_{j=1}^{m} a_{i_{1 j}} \xi_{j i}\right)^{2}\right]=E\left[\sum_{j=1}^{m}\left(a_{i_{1 j}} \xi_{j i}\right)^{2}+2 \sum_{k \neq j=1}^{m} a_{i_{1 k}} a_{i_{1 j}} \xi_{k i} \xi_{j i}\right] \\
& =\sum_{j=1}^{m} a_{i_{1 j}}^{2} E\left[\xi_{j i}^{2}\right]+2 \sum_{k \neq j=1}^{m} a_{i_{1 k}} a_{i_{1 j}} E\left[\xi_{k i}\right] E\left[\xi_{j i}\right]=\sum_{j=1}^{m} a_{i_{1 j}}^{2} \\
E\left[b_{12}\right] & =E\left[\sum_{j=1}^{m} a_{i_{1 j}} \xi_{j i} \sum_{j=1}^{m} a_{i_{2 j}} \xi_{j i}\right]=E\left[\sum_{j=1}^{m} a_{i_{1 j}} a_{i_{2 j}} \xi_{j i}^{2}+\sum_{k \neq j=1}^{m} a_{i_{1 k}} a_{i_{2 j}} \xi_{k} \xi_{j i}\right] \\
& =\sum_{j=1}^{m} a_{i_{1 j}} a_{i_{2 j}} E\left[\xi_{j i}^{2}\right]+\sum_{k \neq j=1}^{m} a_{i_{1 k}} a_{i_{2 j}} E\left[\xi_{k i}\right] E\left[\xi_{j i}\right]=\sum_{j=1}^{m} a_{i_{1 j}} a_{i_{2 j}}=0
\end{aligned}
$$

a igualdade de acima surge de (3.5) e dos valores de $E\left[\xi_{i}\right]$ na definição (3.2).

Analogamente as derivações de $E\left[b_{11}\right]$ e $E\left[b_{12}\right]$ temos que,

$$
\begin{aligned}
& E\left[b_{13}\right]=E\left[\sum_{j=1}^{m} a_{i_{1 j}} \xi_{j i} \sum_{j=1}^{m} a_{i_{3 j}} \xi_{j i}\right]=0, \quad E\left[b_{22}\right]=E\left[\left(\sum_{j=1}^{m} a_{i_{2 j}} \xi_{j i}\right)^{2}\right]=\sum_{j=1}^{m} a_{i_{2 j}}^{2}, \\
& E\left[b_{23}\right]=E\left[\sum_{j=1}^{m} a_{i_{2 j}} \xi_{j i} \sum_{j=1}^{m} a_{i_{3 j}} \xi_{j i}\right]=0, \quad E\left[b_{33}\right]=E\left[\left(\sum_{j=1}^{m} a_{i_{3 j}} \xi_{j i}\right)^{2}\right]=\sum_{j=1}^{m} a_{i_{3 j}}^{2} .
\end{aligned}
$$


Portanto, temos que,

$$
E\left[\mathbf{r}_{i}^{*} \mathbf{r}_{i}^{* T}\right]=\operatorname{diag}\left\{\sum_{j=1}^{m} a_{i_{1 j}}^{2}, \sum_{j=1}^{m} a_{i_{2 j}}^{2}, \sum_{j=1}^{m} a_{i_{3 j}}^{2}\right\}=\Psi_{i} .
$$

Consideramos agora,

$$
\mathbf{r}_{i}^{*} \mathbf{r}_{i}^{* T} \mathbf{r}_{i}^{*}=v_{i}^{3}\left(c_{11}, c_{21}, c_{13}\right)^{T}
$$

onde,

$$
\begin{aligned}
c_{11} & =\left[\sum_{j=1}^{m} a_{i_{1 j}} \xi_{j i}\right]^{3}+\sum_{j=1}^{m} a_{i_{1 j}} \xi_{j i}\left[\sum_{j=1}^{m} a_{i_{2 j}} \xi_{j i}\right]^{2}+\sum_{j=1}^{m} a_{i_{1 j}} \xi_{j i}\left[\sum_{j=1}^{m} a_{i_{13 j}} \xi_{j i}\right]^{2} \\
c_{21} & =\left[\sum_{j=1}^{m} a_{i_{1 j}} \xi_{j i}\right]^{2} \sum_{j=1}^{m} a_{i_{2 j}} \xi_{j i}+\left[\sum_{j=1}^{m} a_{i_{2 j}} \xi_{j i}\right]^{3}+\sum_{j=1}^{m} a_{i_{1 j}} \xi_{j i}\left[\sum_{j=1}^{m} a_{i_{3 j}} \xi_{j i}\right]^{2} \\
c_{31} & =\left[\sum_{j=1}^{m} a_{i_{1 j}} \xi_{j i}\right]^{2} \sum_{j=1}^{m} a_{i_{3 j}} \xi_{j i}+\left[\sum_{j=1}^{m} a_{i_{2 j}} \xi_{j i}\right]^{2} \sum_{j=1}^{m} a_{i_{3 j}} \xi_{j i}+\left[\sum_{j=1}^{m} a_{i_{3 j}} \xi_{j i}\right]^{3} .
\end{aligned}
$$

Calculando o valor esperado, temos,

$$
E\left[\mathbf{r}_{i}^{*} \mathbf{r}_{i}^{* T} \mathbf{r}_{i}^{*}\right]=E\left[v_{i}^{3}\right]\left(E\left[c_{11}\right], E\left[c_{21}\right], E\left[c_{13}\right]\right)^{T}=\gamma\left(E\left[c_{11}\right], E\left[c_{21}\right], E\left[c_{13}\right]\right)^{T}
$$

com,

$$
\begin{aligned}
E\left[c_{11}\right] & =E\left[\left[\sum_{j=1}^{m} a_{i_{1 j}} \xi_{j i}\right]^{3}+\sum_{j=1}^{m} a_{i_{1 j}} \xi_{j i}\left[\sum_{j=1}^{m} a_{i_{2 j}} \xi_{j i}\right]^{2}+\sum_{j=1}^{m} a_{i_{1 j}} \xi_{j i}\left[\sum_{j=1}^{m} a_{i_{13 j}} \xi_{j i}\right]^{2}\right] \\
& =E\left[\left[\sum_{j=1}^{m} a_{i_{1 j}} \xi_{j i}\right]^{3}+E\left[\sum_{j=1}^{m} a_{i_{1 j}} \xi_{j i}\left[\sum_{j=1}^{m} a_{i_{2 j}} \xi_{j i}\right]^{2}\right]+E\left[\sum_{j=1}^{m} a_{i_{1 j}} \xi_{j i}\left[\sum_{j=1}^{m} a_{i_{13 j}} \xi_{j i}\right]^{2}\right] .\right.
\end{aligned}
$$

Analisando os termos em (3.9) temos que,

$$
\left[\sum_{j=1}^{m} a_{i_{1 j}} \xi_{j i}\right]^{3}=\sum_{j=1}^{m} a_{i_{1 j}}^{3} \xi_{j i}^{3}+3 \sum_{j=1}^{m} a_{i_{1 j}}^{2} \xi_{j i}^{2}\left[\sum_{k \neq j, k=1}^{m} a_{i_{1 k}} \xi_{k i}\right]+3\left[\prod_{k<j, k=1}^{m} a_{i_{1 k}} \xi_{k i}+\prod_{j=1}^{m} a_{i_{1 j}} \xi_{j i}\right.
$$

de modo que,

$$
\begin{aligned}
E\left[c_{11}\right]= & E\left[\sum_{j=1}^{m} a_{i_{1 j}}^{3} \xi_{j i}^{3}+3 \sum_{j=1}^{m} a_{i_{1 j}}^{2} \xi_{j i}^{2}\left[\sum_{k \neq j, k=1}^{m} a_{i_{1 k}} \xi_{k i}\right]+3 !\left[\prod_{k<j, k=1}^{m} a_{i_{1 k}} \xi_{k i}+\prod_{j=1}^{m} a_{i_{1 j}} \xi_{j i}\right]\right] \\
= & \sum_{j=1}^{m} a_{i_{1 j}}^{3} E\left[\xi_{j i}^{3}\right]+3 \sum_{j=1}^{m} a_{i_{1 j}}^{2} E\left[\xi_{j i}^{2}\left\{\sum_{k \neq j, k=1}^{m} a_{i_{1 k}} \xi_{k i}\right\}\right]+3 !\left[\prod_{k<j, k=1}^{m} a_{i_{1 k}} E\left[\xi_{k i}\right]+\right. \\
& +\prod_{j=1}^{m} a_{i_{1 j}} E\left[\xi_{j i}\right] \\
= & \sum_{j=1}^{m} a_{i_{1 j}}^{3} E\left[\xi_{j i}^{3}\right]+3 \sum_{j=1}^{m} a_{i_{1 j}}^{2} E\left[\xi_{j i}^{2}\left\{\sum_{k \neq j, k=1}^{m} a_{i_{1 k}} \xi_{k i}\right\}\right]=\sum_{j=1}^{m} a_{i_{1 j}}^{3} \zeta_{j} .
\end{aligned}
$$

Ozán, N. S. 
Analogamente aos cálculos em $E\left[c_{11}\right]$, concluímos que,

$$
E\left[c_{21}\right]=\sum_{j=1}^{m} a_{i_{2 j}}^{3} \zeta_{j}, \quad E\left[c_{31}\right]=\sum_{j=1}^{m} a_{i_{3 j}}^{3} \zeta_{j} .
$$

Logo,

$$
E\left[\mathbf{r}_{i}^{*} \mathbf{r}_{i}^{* T} \mathbf{r}_{i}^{*}\right]=\gamma\left(\sum_{j=1}^{m} a_{i_{1 j}}^{3} \zeta_{j}, \sum_{j=1}^{m} a_{i_{2 j}}^{3} \zeta_{j}, \sum_{j=1}^{m} a_{i_{3 j}}^{3} \zeta_{j}\right)^{T}
$$

Com os resultados obtidos acima fica provada a proposição.

Consideremos, a seguir, o modelo aditivo como em (3.1). Seja $Z_{1}, Z_{2}, \ldots$ seqüência de vetores aleatórios independentes, tal que $\mathbf{Z}_{i}$ tem distribuição assimétrica, com parâmetro de locação $\mu^{*}$. De modo que, $\mu^{*}=\mathrm{a}^{*}+\mathrm{B} \eta$, onde $\mathrm{a}^{*}=\mathrm{a}+\mathrm{B} \eta^{*}$, com $\eta^{*}=E\left[\mathrm{r}^{*}\right] \mathrm{e}$ $\boldsymbol{\eta}=\left(\mu_{x}, 0,0\right)^{T}$. Portanto, se $E\left[\mathbf{r}^{*}\right]=0$, temos que,

$$
\mu^{*}=\left(\begin{array}{c}
\alpha+\beta \mu_{x} \\
\mu_{x}
\end{array}\right)
$$

A matriz de covariâncias $\Sigma_{i}$, é tal que, $\Sigma_{i}=\mathbf{B} \operatorname{Var}\left[\mathbf{r}_{i}\right] \mathbf{B}^{T}$, ou seja,

$$
\Sigma_{i}=\left(\begin{array}{cc}
\beta^{2} \sigma_{x_{i}}^{2}+\sigma_{e_{i}}^{2} & \beta \sigma_{x_{i}}^{2} \\
\beta \sigma_{x_{i}}^{2} & \sigma_{x_{i}}^{2}+\sigma_{u_{i}}^{2}
\end{array}\right) .
$$

Seja $\epsilon_{1}, \ldots, \epsilon_{n}$, uma amostra de $n$ observações independentes, tal que, $\epsilon_{i}=\mathrm{Z}_{i}-\mu^{*}$. Então temos que, $\epsilon_{i}=\mathrm{Z}_{i}-\mu^{*}=\mathrm{a}+\mathrm{Br}_{i}-\mathrm{a}^{*}-\mathrm{B} \boldsymbol{\eta}=\mathrm{B}\left(\mathrm{r}_{i}-\boldsymbol{\eta}-\boldsymbol{\eta}^{*}\right)=\mathrm{B}\left(\mathrm{r}_{i}-\boldsymbol{\eta}\right)$. Portanto,

$$
\epsilon_{i}=\left(\begin{array}{c}
\beta\left(x_{i}-\mu_{x}\right)+e_{i} \\
x_{i}-\mu_{x}+u_{i}
\end{array}\right), \quad i=1, \ldots, n .
$$

Se $\epsilon_{i}=\mathrm{B}\left(\mathrm{r}_{i}-\eta\right)$, e usando (3.2.), temos que, $\boldsymbol{\epsilon}_{i}=\mathbf{B}\left(v_{i} \mathbf{A}_{i} \boldsymbol{\xi}_{i}\right)$. Operando algebricamente temos que, $\boldsymbol{\epsilon}_{i}=v_{i}\left(\mathbf{B A}_{i}\right) \boldsymbol{\xi}_{i}$. Se definimos $\mathbf{T}_{i}=\mathbf{B} \mathbf{A}_{i}$, então,

$$
\epsilon_{i}=v_{i} \mathbf{T}_{i} \boldsymbol{\xi}_{i}, \quad i=1, \ldots, n
$$

onde,

- $\xi_{i}=\left(\xi_{1 i}, \ldots, \xi_{m i}\right)^{T}$, tal que $\xi_{1 i}, \ldots, \xi_{m i}$ são variáveis aleatórias independentes com $E\left[\xi_{j i}\right]=0, E\left[\xi_{j i}^{2}\right]=1, E\left[\xi_{j i}^{3}\right]=\zeta_{j}$ e $E\left[\xi_{j i}^{4}\right]=\kappa_{j}, j=1, \ldots, m$, e $i=1, \ldots, n$,

- $v_{i}$ variável aleatória independente de $\xi_{i} \operatorname{com} E\left[v_{i}^{2}\right]=1, E\left[v_{i}^{3}\right]=\gamma$ e $E\left[v_{i}^{4}\right]=\delta$, e 
- $\mathbf{T}_{i}=\mathbf{B A}_{i}$ matriz de dimensão $2 \times m$, com posto 2 , tal que $\mathbf{T}_{i}=\left(t_{i_{p j}}\right), p=1,2$, $j=1, \ldots, m$ e $i=1, \ldots, n$, com $\mathbf{T}_{i} \mathbf{T}_{i}^{T}=\boldsymbol{\Sigma}_{i}$, com $\boldsymbol{\Sigma}_{i}$ dada em (3.12).

Portanto

$$
\mathbf{T}_{i}=\left(\begin{array}{cccc}
\beta a_{i_{11}}+a_{i_{21}} & \beta a_{i_{12}}+a_{i_{22}} & \ldots & \beta a_{i_{1 m}}+a_{i_{2 m}} \\
a_{i_{11}}+a_{i_{31}} & a_{i_{12}}+a_{i_{32}} & \ldots & a_{i_{1 m}}+a_{i_{3 m}}
\end{array}\right) .
$$

Assim,

$$
\mathbf{T}_{i}=\left(\begin{array}{cccc}
t_{i_{11}} & t_{i_{12}} & \ldots & t_{i_{1 m}} \\
t_{i_{21}} & t_{i_{22}} & \ldots & t_{i_{2 m}}
\end{array}\right)
$$

Então, definimos $\mathbf{t}_{j}$, a j-ésima coluna de $\mathbf{T}_{i}$, para $j=1, \ldots, m$ e $i=1, \ldots, n$, como:

$$
\mathbf{t}_{j}=\left(\begin{array}{c}
t_{i_{1 j}} \\
t_{i_{2 j}}
\end{array}\right)=\left(\begin{array}{c}
\beta a_{i_{1 j}}+a_{i_{2 j}} \\
a_{i_{1 j}}+a_{i_{3 j}}
\end{array}\right)
$$

de modo que,

$$
\Sigma_{i}=\mathbf{T}_{i} \mathbf{T}_{i}^{T}=\left(\begin{array}{cc}
\sum_{j=1}^{m} t_{i_{1 j}}^{2} & \sum_{j=1}^{m} t_{i_{1 j}} t_{i_{2 j}} \\
\sum_{j=1}^{m} t_{i_{1 j}} t_{i_{2 j}} & \sum_{j=1}^{m} t_{i_{2 j}}^{2}
\end{array}\right)=\left(\begin{array}{cc}
\beta^{2} \sigma_{x_{i}}^{2}+\sigma_{e_{i}}^{2} & \beta \sigma_{x_{i}}^{2} \\
\beta \sigma_{x_{i}}^{2} & \sigma_{x_{i}}^{2}+\sigma_{u_{i}}^{2}
\end{array}\right)
$$

e desta última expressão concluímos que,

$$
\begin{aligned}
& \beta^{2} \sigma_{x_{i}}^{2}+\sigma_{e_{i}}^{2}=\sum_{j=1}^{m} t_{i_{1 j}}^{2}=\sum_{j=1}^{m}\left(\beta a_{i_{1 j}}+a_{i_{2 j}}\right)^{2}, \\
& \beta \sigma_{x_{i}}^{2}=\sum_{j=1}^{m} t_{i_{1 j}} t_{i_{2 j}}=\sum_{j=1}^{m}\left(\beta a_{i_{1 j}}+a_{i_{2 j}}\right)\left(a_{i_{1 j}}+a_{i_{3 j}}\right) \text {, } \\
& \sigma_{x_{i}}^{2}+\sigma_{u_{i}}^{2}=\sum_{j=1}^{m} t_{i_{2 j}}^{2}=\sum_{j=1}^{m}\left(a_{i_{1 j}}+a_{i_{2 j}}\right)^{2} .
\end{aligned}
$$

A expressão para o parâmetro de assimetria marginal, é

$$
\begin{aligned}
& \lambda_{1 i}^{*}=\lambda_{i}\left(\beta\left(x_{i}-\mu_{x}\right)+e_{i}\right)=\gamma \sum_{j=1}^{m} \frac{t_{i_{1 j}}^{3} \zeta_{j}}{\sigma_{i i}^{\frac{3}{2}}}, \\
& \lambda_{2 i}^{*}=\lambda_{i}\left(x_{i}-\mu_{x_{i}}+u_{i}\right)=\gamma \sum_{j=1}^{m} \frac{t_{i_{2 j}}^{3} \zeta_{j}}{\sigma_{i i}^{\frac{3}{2}}} .
\end{aligned}
$$

No caso do parâmetro de curtose, temos que,

$$
\begin{aligned}
& \kappa_{1 i}^{*}=\kappa_{i}\left(\beta\left(x_{i}-\mu_{x}\right)+e_{i}\right)=\delta\left\{\sum_{j=1}^{m} \frac{t_{i_{1 j}}^{4}\left(\kappa_{j}-3\right)}{\sigma_{i i}^{2}}+3\right\}, \\
& \kappa_{2 i}^{*}=\kappa_{i}\left(x_{i}-\mu_{x_{i}}+u_{i}\right)=\delta\left\{\sum_{j=1}^{m} \frac{t_{i_{2 j}}^{4}\left(\kappa_{j}-3\right)}{\sigma_{i i}^{2}}+3\right\} .
\end{aligned}
$$


Portanto, se $\boldsymbol{\epsilon}_{i}=v_{i} \mathbf{T}_{i} \boldsymbol{\xi}_{i}, i=1, \ldots, n$, segundo a equação (3.13), temos que os $\epsilon_{i}$ são independentes e identicamente distribuídos, com distribuição assimétrica. Ou seja, $\epsilon_{i} \stackrel{i i d}{\sim} S\left(0, \Sigma_{i}, \lambda_{i}^{*}, \kappa_{i}^{*}\right)$, onde $\lambda_{i}^{*}=\left(\lambda_{1 i}^{*}, \lambda_{2 i}^{*}\right)^{T}$ e $\kappa_{i}^{*}=\left(\kappa_{1 i}^{*}, \kappa_{2 i}^{*}\right)$ cujas expressões são dadas em (3.15) e (3.16), respectivamente, $i=1, \ldots, n$.

Seja $\mathbf{Z}_{1}, \ldots, \mathbf{Z}_{n}$, uma amostra de $n$ observações independentes. Sejam a média da amostra, $\overline{\mathbf{Z}}=\frac{1}{n} \sum_{i=1}^{n}$ e a matriz de covariâncias amostrais, $\mathbf{S}_{Z}=\frac{1}{n} \sum_{i=1}^{n}(\mathbf{Z}-\overline{\mathbf{Z}})(\mathbf{Z}-\overline{\mathbf{Z}})^{T}$, a seguir, estudamos as respectivas propriedades assintóticas, consideramos um modelo com variância constante.

\subsubsection{Modelo Estrutural Assimétrico Linear Aditivo com variância constante}

Nesta seção estudamos um caso particular do modelo considerado na seção anterior, onde $\Psi_{i}=\Psi, i=1, \ldots, n$. No modelo estrutural assimétrico com a variância constante temos, $\mathbf{A}_{i}=\mathbf{A}$, para todo $i=1, \ldots, n$, de modo que,

$$
\mathbf{r}_{i}-\boldsymbol{\eta}=\left(x_{i}-\mu_{x}, e_{i}, u_{i}\right)^{T}=v_{i} \mathbf{A} \xi_{i}
$$

com $\mathbf{A}=\left(a_{i j}\right)$ matriz de dimensão $3 \times m$, com posto de $\mathbf{A}$ igual a 3 , tal que $\mathbf{A A}^{T}=\mathbf{\Psi}$. Portanto,

$$
\left(\begin{array}{c}
x_{i}-\mu_{x} \\
e_{i} \\
u_{i}
\end{array}\right)=\left(\begin{array}{c}
v_{i} \sum_{j=1}^{m} a_{1 j} \xi_{j i} \\
v_{i} \sum_{j=1}^{m} a_{2 j} \xi_{j i} \\
v_{i} \sum_{j=1}^{m} a_{3 j} \xi_{j i}
\end{array}\right)
$$

Isto implica que na equação (3.13) temos,

$$
\epsilon_{i}=v_{i} \mathbf{T} \xi_{i}
$$

com $\mathbf{T}=\left(t_{i j}\right)$ matriz de dimensão $2 \times m$, com posto 2 , tal que $\mathbf{T T}^{T}=\boldsymbol{\Sigma}$ e $\mathbf{T}=\mathbf{B A}$. Assim,

$$
\mathbf{T}=\left(\begin{array}{cccc}
t_{11} & t_{12} & \ldots & t_{1 m} \\
t_{21} & t_{22} & \ldots & t_{2 m}
\end{array}\right)
$$

Então, $\mathbf{t}_{j}$, a j-ésima columna de $\mathbf{T}$, pode ser escrita como

$$
\mathbf{t}_{j}=\left(\begin{array}{c}
t_{1 j} \\
t_{2 j}
\end{array}\right)=\left(\begin{array}{c}
\beta a_{1 j}+a_{2 j} \\
a_{1 j}+a_{3 j}
\end{array}\right), \quad j=1, \ldots, m .
$$

Ozán, N. S. 
Portanto,

$$
\boldsymbol{\Sigma}=\mathbf{T T}^{T}=\left(\begin{array}{cc}
\sum_{j=1}^{m} t_{1 j}^{2} & \sum_{j=1}^{m} t_{1 j} t_{2 j} \\
\sum_{j=1}^{m} t_{1 j} t_{2 j} & \sum_{j=1}^{m} t_{2 j}^{2}
\end{array}\right)=\left(\begin{array}{cc}
\beta^{2} \sigma_{x}^{2}+\sigma_{e}^{2} & \beta \sigma_{x}^{2} \\
\beta \sigma_{x}^{2} & \sigma_{x}^{2}+\sigma_{u}^{2}
\end{array}\right)
$$

Derivamos, a seguir, resultados de utilidade no estudo assintótico apresentado.

Lema 3.2. Seja $\boldsymbol{\epsilon}_{i}=v_{i} \mathbf{T} \boldsymbol{\xi}_{i}$, para $i=1, \ldots, n$, segundo a equação (3.18).

Então, $\Gamma=E\left[\epsilon_{i} \operatorname{vech}^{T}\left(\epsilon_{i} \epsilon_{i}^{T}\right)\right], i=1, \ldots, n$, é dada por,

$$
\Gamma=\gamma \sum_{j=1}^{m} \mathbf{T}_{1 j} \zeta_{j}
$$

$\operatorname{com} \gamma=E\left(v_{i}^{3}\right), \zeta_{j}=E\left(\xi_{j i}^{3}\right), j=1, \ldots, m, e$

$$
\mathbf{T}_{1 j}=\left(\begin{array}{ccc}
t_{1 j}^{3} & t_{1 j}^{2} t_{2 j} & t_{1 j} t_{2 j}^{2} \\
t_{1 j}^{2} t_{2 j} & t_{1 j} t_{2 j}^{2} & t_{2 j}^{3}
\end{array}\right)
$$

Demonstração. Se $\epsilon_{i}=Z_{i}-\mu=\mathrm{B}\left(\mathrm{r}_{i}-\eta\right)$, então,

$$
\operatorname{vech}\left(\epsilon_{i} \epsilon_{i}^{T}\right)=\left(\begin{array}{c}
{\left[\beta\left(x_{i}-\mu_{x}\right)+e_{i}\right]^{2}} \\
{\left[x_{i}-\mu_{x}+u_{i}\right]^{2}}
\end{array}\right) .
$$

Portanto,

$$
\epsilon_{i} \operatorname{vech}^{T}\left(\epsilon_{i} \epsilon_{i}^{T}\right)=\left(\begin{array}{lll}
E_{11} & E_{12} & E_{13} \\
E_{21} & E_{22} & E_{23}
\end{array}\right)
$$

de modo que,

$$
\begin{array}{ll}
E_{11}=\left[\beta\left(x_{i}-\mu_{x}\right)+e_{i}\right]^{3}, & E_{21}=\left[\beta\left(x_{i}-\mu_{x}\right)+e_{i}\right]^{2}\left(x_{i}-\mu_{x}+u_{i}\right), \\
E_{12}=\left[\beta\left(x_{i}-\mu_{x}\right)+e_{i}\right]^{2}\left(x_{i}-\mu_{x}+u_{i}\right), & E_{22}=\left[\beta\left(x_{i}-\mu_{x}\right)+e_{i}\right]\left(x_{i}-\mu_{x}+u_{i}\right)^{2}, \\
E_{13}=\left[\beta\left(x_{i}-\mu_{x}\right)+e_{i}\right]\left(x_{i}-\mu_{x}+u_{i}\right)^{2}, & E_{23}=\left[x_{i}-\mu_{x}+u_{i}\right]^{3} .
\end{array}
$$

Calculando o valor esperado, temos que,

$$
\Gamma=E\left[\epsilon_{i} v e c h^{T}\left(\epsilon_{i} \epsilon_{i}^{T}\right)\right]=\left(\begin{array}{lll}
E\left[E_{11}\right] & E\left[E_{12}\right] & E\left[E_{13}\right] \\
E\left[E_{21}\right] & E\left[E_{22}\right] & E\left[E_{23}\right]
\end{array}\right)
$$


Portanto, aplicando (3.5) e manipulando algebricamente, temos que,

$$
\begin{aligned}
E\left[\left\{\beta\left(x_{i}-\mu_{x}\right)+e_{i}\right\}^{3}\right]= & E\left[\left\{\beta\left(x_{i}-\mu_{x}\right)\right\}^{3}+3\left\{\beta\left(x_{i}-\mu_{x}\right)\right\}^{2} e_{i}+3 \beta\left(x_{i}-\mu_{x}\right) e_{i}^{2}+e_{i}^{3}\right] \\
= & E\left[\left\{\beta\left(x_{i}-\mu_{x}\right)\right\}^{3}\right]+E\left[e_{i}^{3}\right] \\
= & \left.\gamma \sum_{j=1}^{m} \beta^{3} a_{1 j}^{3} \zeta_{j}+\gamma \sum_{j=1}^{m} a_{2 j}^{3}\right) \zeta_{j}=\gamma \sum_{j=1}^{m}\left(\beta^{3} a_{1 j}^{3}+a_{2 j}^{3}\right) \zeta_{j}, \\
= & E\left[\left\{\beta\left(x_{i}-\mu_{x}\right)\right\}^{3}+3\left\{\beta\left(x_{i}-\mu_{x}\right)\right\}^{2} u_{i}+3 \beta\left(x_{i}-\mu_{x}\right) u_{i}^{2}+u_{i}^{3}\right] \\
= & E\left[\left\{x_{i}-\mu_{x}\right\}^{3}\right]+E\left[u_{i}^{3}\right] \\
\left.=\gamma\left[x_{i}-\mu_{x}+u_{i}\right\}^{3}\right]= & \gamma \sum_{j=1}^{m} a_{1 j}^{3} \zeta_{j}+\gamma \sum_{j=1}^{m} a_{3 j}^{3} \zeta_{j}=\gamma \sum_{j=1}^{m}\left(a_{1 j}^{3}+a_{3 j}^{3}\right) \zeta_{j}, \\
= & E\left[\left\{\beta\left(x_{1}-\mu_{x}\right)+e_{i}\right\}^{2}\left(x_{i}-\mu_{x}\right)\right]+ \\
& \left.+E\left[\left\{\beta\left(x_{1}-\mu_{x}\right)+e_{i}\right\}^{2} u_{i}\right)\right] \\
= & E\left[\beta^{2}\left(x_{1}-\mu_{x}\right)^{3}\right]=\gamma \beta^{2} \sum_{j=1}^{m} a_{1 j}^{3} \zeta_{j}, \\
E\left[\left\{\beta\left(x_{1}-\mu_{x}\right)+e_{i}\right\}^{2}\left(x_{i}-\mu_{x}+u_{i}\right)\right]= & \\
& \\
& +E\left[\left\{\beta\left(x_{i}-\mu_{x}\right)\right\}\left\{x_{i}-\mu_{x}+u_{i}\right\}^{2}\right]+ \\
= & E\left[\beta\left(x_{i}-\mu_{x}\right)^{3}\right]=\gamma \beta \sum_{j=1}^{m} a_{1 j}^{3} \zeta^{j} .
\end{aligned}
$$

Assim, a matriz $\Gamma$ tem a seguinte expressão,

$$
\boldsymbol{\Gamma}=\gamma\left(\begin{array}{ccc}
\sum_{j=1}^{m}\left(\beta^{3} a_{1 j}^{3}+a_{2 j}^{3}\right) \zeta_{j} & \beta^{2} \sum_{j=1}^{m} a_{1 j}^{3} \zeta_{j} & \beta \sum_{j=1}^{m} a_{1 j}^{3} \zeta_{j} \\
\beta^{2} \sum_{j=1}^{m} a_{1 j}^{3} \zeta_{j} & \beta \sum_{j=1}^{m} a_{1 j}^{3} \zeta_{j} & \sum_{j=1}^{m}\left(a_{1 j}^{3}+a_{3 j}^{3}\right) \zeta_{j}
\end{array}\right),
$$

observando que (3.22) é função da estrutura de covariâncias assumida para $\mathbf{r}_{i}^{*}$, ou seja, depende da matriz A. Por outro lado, como,

$$
\epsilon_{i}=\left(\begin{array}{c}
\beta\left(x_{i}-\mu_{x}\right)+e_{i} \\
x_{i}-\mu_{x_{i}}+u_{i}
\end{array}\right)=v_{i} \mathbf{T} \boldsymbol{\xi}_{i}=\left(\begin{array}{c}
v_{i} \sum_{j=1}^{m} t_{1 j} \xi_{j i} \\
v_{i} \sum_{j=1}^{m} t_{2 j} \xi_{j i}
\end{array}\right),
$$

temos que,

$$
\beta\left(x_{i}-\mu_{x}\right)+e_{i}=v_{i} \sum_{j=1}^{m} t_{1 j} \xi_{j i}, \quad \text { e } \quad x_{i}-\mu_{x_{i}}+u_{i}=v_{i} \sum_{j=1}^{m} t_{2 j} \xi_{j i} .
$$

Ozán, N. S. 
Portanto,

- $E\left[\left\{\beta\left(x_{i}-\mu_{x}\right)+e_{i}\right\}^{3}\right]=E\left[\left\{v_{i} \sum_{j=1}^{m} t_{1 j} \xi_{j i}\right\}^{3}\right]=\gamma \sum_{j=1}^{m} t_{1 j}^{3} \zeta_{j}$

- $E\left[\left\{x_{i}-\mu_{x}+u_{i}\right\}^{3}\right]=E\left[\left\{v_{i} \sum_{j=1}^{m} t_{2 j} \xi_{j i}\right\}^{3}\right]=\gamma \sum_{j=1}^{m} t_{2 j}^{3} \zeta_{j}$

- $E\left[\left\{\beta\left(x_{i}-\mu_{x}\right)+e_{i}\right\}^{2}\left\{x_{i}-\mu_{x}+u_{i}\right\}\right]=E\left[\left\{v_{i} \sum_{j=1}^{m} t_{1 j} \xi_{j i}\right\}^{2}\left\{v_{i} \sum_{j=1}^{m} t_{2 j} \xi_{j i}\right\}\right]=\gamma \sum_{j=1}^{m} t_{1 j}^{2} t_{2 j} \zeta_{j}$,

- $E\left[\left\{\beta\left(x_{i}-\mu_{x}\right)+e_{i}\right\}\left\{x_{i}-\mu_{x}+u_{i}\right\}^{2}\right]=E\left[\left\{v_{i} \sum_{j=1}^{m} t_{1 j} \xi_{j i}\right\}\left\{v_{i} \sum_{j=1}^{m} t_{2 j} \xi_{j i}\right\}^{2}\right]=\gamma \sum_{j=1}^{m} t_{1 j} t_{2 j}^{2} \zeta_{j}$,

usando os resultados obtidos acima em (3.22), temos que,

$$
\boldsymbol{\Gamma}=\gamma\left(\begin{array}{ccc}
\sum_{j=1}^{m} t_{1 j}^{3} \zeta_{j} & \sum_{j=1}^{m} t_{1 j}^{2} t_{2 j} \zeta_{j} & \sum_{j=1}^{m} t_{1 j} t_{2 j}^{2} \zeta_{j} \\
\sum_{j=1}^{m} t_{1 j}^{2} t_{2 j} \zeta_{j} & \sum_{j=1}^{m} t_{1 j} t_{2 j}^{2} \zeta_{j} & \sum_{j=1}^{m} t_{2 j}^{3} \zeta_{j}
\end{array}\right)=\gamma \sum_{j=1}^{m}\left(\begin{array}{ccc}
t_{1 j}^{3} & t_{1 j}^{2} t_{2 j} & t_{1 j} t_{2 j}^{2} \\
t_{1 j}^{2} t_{2 j} & t_{1 j} t_{2 j}^{2} & t_{2 j}^{3}
\end{array}\right) \zeta_{j},
$$

e definindo $\mathbf{T}_{1 j}=\left(\begin{array}{ccc}t_{1 j}^{3} & t_{1 j}^{2} t_{2 j} & t_{1 j} t_{2 j}^{2} \\ t_{1 j}^{2} t_{2 j} & t_{1 j} t_{2 j}^{2} & t_{2 j}^{3}\end{array}\right)$, a matriz $\boldsymbol{\Gamma}$ tem a expressão (3.20), o que prova o lema.

Observação 3. Considerando a expressão (3.22) para $\boldsymbol{\Gamma}$, e manipulando algebricamente, escrevemos,

$$
\Gamma=\gamma \sum_{j=1}^{m}\left[a_{1 j}^{3}\left(\begin{array}{ccc}
\beta^{3} & \beta^{2} & \beta \\
\beta^{2} & \beta & 1
\end{array}\right)+\left(\begin{array}{lll}
a_{2 j}^{3} & 0 & 0 \\
0 & 0 & a_{3 j}^{3}
\end{array}\right)\right] \zeta_{j}
$$

Observação 4. Se consideramos que $\epsilon_{i}, i=1, \ldots, n$, tem distribuição elíptica, então, $E\left[\xi_{j i}^{3}\right]=\zeta_{j}=0$ e $\boldsymbol{\Gamma}=0$, resultando o caso simétrico.

Lema 3.3. Seja $\boldsymbol{\epsilon}_{i}=v_{i} \mathbf{T} \boldsymbol{\xi}_{i}, i=1, \ldots, n$, segundo a equação (3.18).

Então, $\Lambda=\operatorname{Var}\left[\operatorname{vech}\left(\epsilon_{i} \epsilon_{i}^{T}\right)\right]$, é uma matriz simétrica, de dimensão $3 \times 3$, dada por,

$$
\Lambda=(3 \delta-1) \mathbf{T}^{*}+\delta \sum_{j=1}^{m}\left(\kappa_{j}-3\right) \mathbf{T}_{2 j}
$$

Ozán, N. S. 
com,

$$
\mathbf{T}^{*}=\left(\begin{array}{ccc}
\left(\sum_{j=1}^{m} t_{1 j}^{2}\right)^{2} & \left(\sum_{j=1}^{m} t_{1 j}^{2}\right)\left(\sum_{j=1}^{m} t_{1 j} t_{2 j}\right) & \left(\sum_{j=1}^{m} t_{1 j}^{2}\right)\left(\sum_{j=1}^{m} t_{2 j}^{2}\right) \\
\cdots & \left(\sum_{j=1}^{m} t_{1 j} t_{2 j}\right)^{2} & \left(\sum_{j=1}^{m} t_{1 j} t_{2 j}\right)\left(\sum_{j=1}^{m} t_{2 j}^{2}\right) \\
\cdots & \cdots & \left(\sum_{j=1}^{m} t_{2 j}^{2}\right)^{2}
\end{array}\right)
$$

$e$

$$
\mathbf{T}_{2 j}=\left(\begin{array}{ccc}
t_{1 j}^{4} & t_{1 j}^{3} t_{2 j} & t_{1 j}^{2} t_{2 j}^{2} \\
\ldots & t_{1 j}^{2} t_{2 j}^{2} & t_{1 j} t_{2 j}^{3} \\
\cdots & \cdots & t_{2 j}^{4}
\end{array}\right)
$$

Demonstração. Usando o Teorema 2.1, a matriz $\Lambda=\operatorname{Var}\left[\operatorname{vech}\left(\epsilon_{i} \epsilon_{i}^{T}\right)\right]$ é dada por, $\Lambda=2 \delta \mathrm{D}_{2}^{+}(\Sigma \otimes \Sigma) \mathbf{D}_{2}^{+T}+(\delta-1) \operatorname{vech}(\Sigma) \operatorname{vech}^{T}(\Sigma)+\delta \sum_{j=1}^{m}\left(\kappa_{j}-3\right) \operatorname{vech}\left(t_{j} t_{j}^{T}\right) \operatorname{vech}^{T}\left(t_{j} t_{j}^{T}\right)$ com $t_{j}$ a j-ésima coluna da matriz $\mathbf{T}=\mathbf{B} \mathbf{A}, j=1, \ldots, m$.

Considerando os termos da soma acima por partes, temos que,

1. $2 \delta \mathbf{D}_{2}^{+}(\Sigma \otimes \Sigma) \mathbf{D}_{2}^{+T}$.

Existe a matriz $\mathbf{D}_{2}$ de dimensão $4 \times 3$, com posto $r\left(\mathbf{D}_{2}\right)=3$, tal que,

$$
\operatorname{vec}(\boldsymbol{\Sigma})=\mathbf{D}_{2} \operatorname{vech}(\Sigma),
$$

e $\mathbf{D}_{2}^{+}=\left(\mathbf{D}_{2}^{T} \mathbf{D}_{2}\right)^{-1} \mathbf{D}_{2}^{T}$ (Muirhead, 1982; Magnus e Neudecker, 1988).

Portanto, uma matriz $\mathrm{D}_{2}$ que verifica a equação (3.24) é,

$$
\mathbf{D}_{2}=\left(\begin{array}{lll}
1 & 0 & 0 \\
0 & 1 & 0 \\
0 & 1 & 0 \\
0 & 0 & 1
\end{array}\right) \text {. }
$$

Considerando a matriz $\Sigma$ da equação (3.19) e $\mathrm{D}_{2}^{+}$, temos que,

$$
2 \delta \mathbf{D}_{2}^{+}(\Sigma \otimes \Sigma) \mathbf{D}_{2}^{+T}=2 \delta\left(\begin{array}{ccc}
\left(\sum_{j=1}^{m} t_{1 j}^{2}\right)^{2} & \left(\sum_{j=1}^{m} t_{1 j}^{2}\right)\left(\sum_{j=1}^{m} t_{1 j} t_{2 j}\right) & \left(\sum_{j=1}^{m} t_{1 j}^{2}\right)\left(\sum_{j=1}^{m} t_{2 j}^{2}\right) \\
\cdots & \left(\sum_{j=1}^{m} t_{1 j} t_{2 j}\right)^{2} & \left(\sum_{j=1}^{m} t_{1 j} t_{2 j}\right)\left(\sum_{j=1}^{m} t_{2 j}^{2}\right) \\
\ldots & \ldots & \left(\sum_{j=1}^{m} t_{2 j}^{2}\right)^{2}
\end{array}\right),
$$

que denotamos por $\mathbf{T}^{*}$, de modo que, $2 \delta \mathbf{D}_{2}^{+}(\Sigma \otimes \Sigma) \mathrm{D}_{2}^{+T}=2 \delta \mathbf{T}^{*}$. 
2. Se $\Sigma$ tem a expressão (3.23), então, vech $(\Sigma) \operatorname{vech}^{T}(\Sigma)$ é dado por,

$$
\operatorname{vech}(\Sigma) \operatorname{vech}^{T}(\Sigma)=\left(\begin{array}{ccc}
\left(\sum_{j=1}^{m} t_{1 j}^{2}\right)^{2} & \left(\sum_{j=1}^{m} t_{1 j}^{2}\right)\left(\sum_{j=1}^{m} t_{1 j} t_{2 j}\right) & \left(\sum_{j=1}^{m} t_{1 j}^{2}\right)\left(\sum_{j=1}^{m} t_{2 j}^{2}\right) \\
\ldots & \left(\sum_{j=1}^{m} t_{1 j} t_{2 j}\right)^{2} & \left(\sum_{j=1}^{m} t_{1 j} t_{2 j}\right)\left(\sum_{j=1}^{m} t_{2 j}^{2}\right) \\
\ldots & \cdots & \left(\sum_{j=1}^{m} t_{2 j}^{2}\right)^{2}
\end{array}\right)=\mathbf{T}^{*} .
$$

3. $\delta \sum_{j=1}^{m}\left(\kappa_{j}-3\right) \operatorname{vech}\left(t_{j} t_{j}^{T}\right) \operatorname{vech}^{T}\left(t_{j} t_{j}^{T}\right)$.

$$
\operatorname{vech}\left(t_{j} t_{j}^{\prime}\right) \operatorname{vech}^{\prime}\left(t_{j} t_{j}^{\prime}\right)=\left(\begin{array}{ccc}
t_{1 j}^{4} & t_{1 j}^{3} t_{2 j} & t_{1 j}^{2} t_{2 j}^{2} \\
\ldots & t_{1 j}^{2} t_{2 j}^{2} & t_{1 j} t_{2 j}^{3} \\
\ldots & \cdots & t_{2 j}^{4}
\end{array}\right)=\mathbf{T}_{2 j} .
$$

Logo, somando os termos em (1), (2) e (3), temos que,

$$
\begin{aligned}
\Lambda & =2 \delta \mathbf{T}^{*}+(\delta-1) \mathbf{T}^{*}+\delta \sum_{j=1}^{m}\left(\kappa_{j}-3\right) \mathbf{T}_{2 j} \\
& =(3 \delta-1) \mathbf{T}^{*}+\delta \sum_{j=1}^{m}\left(\kappa_{j}-3\right) \mathbf{T}_{2 j},
\end{aligned}
$$

portanto o lema fica provado.

Observação 5. Podemos escrever os componentes da matriz $\Lambda=\left(\lambda_{i j}\right)$, de dimensão $3 \times 3$, como,

$$
\begin{aligned}
& \lambda_{11}=(3 \delta-1)\left(\sum_{j=1}^{m} t_{1 j}^{2}\right)^{2}+\delta \sum_{j=1}^{m}\left(\kappa_{j}-3\right) t_{1 j}^{4}, \\
& \lambda_{12}=(3 \delta-1) \sum_{j=1}^{m} t_{1 j}^{2} \sum_{j=1}^{m} t_{1 j} t_{2 j}+\delta \sum_{j=1}^{m}\left(\kappa_{j}-3\right) t_{1 j}^{3} t_{2 j}, \\
& \lambda_{13}=(3 \delta-1) \sum_{j=1}^{m} t_{1 j}^{2} \sum_{j=1}^{m} t_{2 j}^{2}+\delta \sum_{j=1}^{m}\left(\kappa_{j}-3\right) t_{1 j}^{2} t_{2 j}^{2}, \\
& \lambda_{22}=(3 \delta-1)\left(\sum_{j=1}^{m} t_{1 j} t_{2 j}\right)^{2}+\delta \sum_{j=1}^{m}\left(\kappa_{j}-3\right) t_{1 j}^{2} t_{2 j}^{2}, \\
& \lambda_{23}=(3 \delta-1) \sum_{j=1}^{m} t_{1 j} t_{2 j} \sum_{j=1}^{m} t_{2 j}^{2}+\delta \sum_{j=1}^{m}\left(\kappa_{j}-3\right) t_{1 j} t_{2 j}^{3}, \\
& \lambda_{33}=(3 \delta-1)\left(\sum_{j=1}^{m} t_{2 j}^{2}\right)^{2}+\delta \sum_{j=1}^{m}\left(\kappa_{j}-3\right) t_{2 j}^{4} .
\end{aligned}
$$


Utilizando a expressão (3.19), temos que,

$$
\begin{aligned}
& \lambda_{11}=(3 \delta-1)\left(\beta^{2} \sigma_{x}^{2}+\sigma_{e}^{2}\right)^{2}+\delta \sum_{j=1}^{m}\left(\kappa_{j}-3\right) t_{1 j}^{4}, \\
& \lambda_{12}=(3 \delta-1)\left(\beta^{2} \sigma_{x}^{2}+\sigma_{e}^{2}\right) \beta \sigma_{x}^{2}+\delta \sum_{j=1}^{m}\left(\kappa_{j}-3\right) t_{1 j}^{3} t_{2 j}, \\
& \lambda_{13}=(3 \delta-1)\left(\beta^{2} \sigma_{x}^{2}+\sigma_{e}^{2}\right)\left(\sigma_{x}^{2}+\sigma_{u}^{2}\right)+\delta \sum_{j=1}^{m}\left(\kappa_{j}-3\right) t_{1 j}^{2} t_{2 j}^{2}, \\
& \lambda_{22}=(3 \delta-1) \beta^{2} \sigma_{x}^{4}+\delta \sum_{j=1}^{m}\left(\kappa_{j}-3\right) t_{1 j}^{2} t_{2 j}^{2}, \\
& \lambda_{23}=(3 \delta-1) \beta \sigma_{x}^{2}\left(\sigma_{x}^{2}+\sigma_{u}^{2}\right)+\delta \sum_{j=1}^{m}\left(\kappa_{j}-3\right) t_{1 j} t_{2 j}^{3}, \\
& \lambda_{33}=(3 \delta-1)\left(\sigma_{x}^{2}+\sigma_{u}^{2}\right)^{2}+\delta \sum_{j=1}^{m}\left(\kappa_{j}-3\right) t_{2 j}^{4},
\end{aligned}
$$

e portanto,

$$
\begin{aligned}
\Lambda= & (3 \delta-1)\left(\begin{array}{ccc}
\left(\beta^{2} \sigma_{x}^{2}+\sigma_{e}^{2}\right)^{2} & \left(\beta^{2} \sigma_{x}^{2}+\sigma_{e}^{2}\right) \beta \sigma_{x}^{2} & \left(\beta^{2} \sigma_{x}^{2}+\sigma_{e}^{2}\right)\left(\sigma_{x}^{2}+\sigma_{u}^{2}\right) \\
\cdots & \beta^{2} \sigma_{x}^{4} & \beta^{2} \sigma_{x}^{4}\left(\sigma_{x}^{2}+\sigma_{u}^{2}\right) \\
\cdots & \cdots & \left(\sigma_{x}^{2}+\sigma_{u}^{2}\right)^{2}
\end{array}\right)+ \\
& +\delta \sum_{j=1}^{m}\left(\kappa_{j}-3\right) \mathbf{T}_{2 j},
\end{aligned}
$$

onde $\mathbf{T}_{2 j}$ definido no lema anterior.

Corolario 3.4. 1. Se $\epsilon_{i}$ tem distribuição pseudo-elíptica, com média zero e matriz de covâriancia $\Sigma$, isto é, $\kappa_{j}=3$, para todo $j=1, \ldots, m$, então, definimos $\Lambda=\Lambda_{E}$ e é dada por,

$$
\Lambda_{E}=(3 \delta-1) \mathbf{T}^{*}
$$

com $\mathrm{T}^{*}$ em (3.23). Além disso, comparando com (3.25), temos que,

$$
\Lambda_{E}=(3 \delta-1)\left(\begin{array}{ccc}
\left(\beta^{2} \sigma_{x}^{2}+\sigma_{e}^{2}\right)^{2} & \left(\beta^{2} \sigma_{x}^{2}+\sigma_{e}^{2}\right) \beta \sigma_{x}^{2} & \left(\beta^{2} \sigma_{x}^{2}+\sigma_{e}^{2}\right)\left(\sigma_{x}^{2}+\sigma_{u}^{2}\right) \\
\cdots & \beta^{2} \sigma_{x}^{4} & \beta^{2} \sigma_{x}^{4}\left(\sigma_{x}^{2}+\sigma_{u}^{2}\right) \\
\cdots & \cdots & \left(\sigma_{x}^{2}+\sigma_{u}^{2}\right)^{2}
\end{array}\right) .
$$

2. Se $\epsilon_{i}$ tem distribuição pseudo-normal, com média zero e matriz de covâriancia $\Sigma$, isto é, $\kappa_{j}=3$, para todo $j=1, \ldots, m$ e $\delta=1$, então, definimos $\Lambda=\Lambda_{N}$ e é dada por,

$$
\Lambda_{N}=2 \mathrm{~T}^{*}
$$


com $\mathrm{T}^{*}$ em (3.23). Além disso, usando (3.25), temos que,

$$
\Lambda_{N}=2\left(\begin{array}{ccc}
\left(\beta^{2} \sigma_{x}^{2}+\sigma_{e}^{2}\right)^{2} & \left(\beta^{2} \sigma_{x}^{2}+\sigma_{e}^{2}\right) \beta \sigma_{x}^{2} & \left(\beta^{2} \sigma_{x}^{2}+\sigma_{e}^{2}\right)\left(\sigma_{x}^{2}+\sigma_{u}^{2}\right) \\
\cdots & \beta^{2} \sigma_{x}^{4} & \beta^{2} \sigma_{x}^{4}\left(\sigma_{x}^{2}+\sigma_{u}^{2}\right) \\
\cdots & \cdots & \left(\sigma_{x}^{2}+\sigma_{u}^{2}\right)^{2}
\end{array}\right) .
$$

De acordo aos resultados obtidos acima, sabemos que,

1. $\epsilon_{i}$ tem distribuição assimétrica, ou seja, $\epsilon_{i} \stackrel{i i d}{\sim} S\left(0, \Sigma, \lambda^{*}, \kappa^{*}\right), i=1, \ldots, n$.

2. $\mathbf{r}_{i}^{*}=\mathbf{r}_{i}-\boldsymbol{\eta}$ tem momento de quarta ordem finito.

Portanto, $\Lambda=\operatorname{Var}\left[\operatorname{vech}\left(\epsilon_{1} \epsilon_{1}^{T}\right)\right]<\infty$.

3. Sejam $\overline{\mathbf{Z}}=\frac{1}{n} \sum_{i=1}^{n} \mathbf{Z}_{i}$ e $\mathbf{S}_{Z}=\frac{1}{n} \sum_{i=1}^{n}\left(\mathbf{Z}_{i}-\overline{\mathbf{Z}}\right)\left(\mathbf{Z}_{i}-\overline{\mathbf{Z}}\right)^{T}$, de modo que se verificam as suposições $A_{1}, A_{2}$ e $A_{3}$ do Lema 2.1 em Arellano-Valle, Bolfarine e Gasco (2002), e portanto temos que, quando $n \rightarrow \infty$,

$$
\overline{\mathrm{Z}} \stackrel{q c}{\rightarrow} \mu^{*} \quad e \quad \mathrm{~S}_{Z} \stackrel{q c}{\rightarrow} \Sigma .
$$

Portanto, verificam-se as hipóteses do Teorema 2.1 em Arellano-Valle et al (2002), de modo que enunciamos o seguinte resultado.

Teorema 3.5. Sob as condições mencionadas acima, temos que,

$$
\left(\begin{array}{c}
\sqrt{n}\left(\overline{\mathbf{Z}}-\boldsymbol{\mu}^{*}\right) \\
\sqrt{n} \operatorname{vech}\left(\mathbf{S}_{Z}-\Sigma\right)
\end{array}\right) \stackrel{d}{\rightarrow} N_{5}\left(\left(\begin{array}{l}
0 \\
0
\end{array}\right) ;\left(\begin{array}{cc}
\Sigma & \Gamma \\
\Gamma^{T} & \Lambda
\end{array}\right)\right)
$$

onde $\boldsymbol{\Sigma}=\mathbf{T T}^{T}$, e $\Gamma$ e $\Lambda$ são obtidas nos Lemas 3.2 e 3.3, respectivamente.

É importante destacar que a distribuição assintótica do vetor de médias e matriz de covâriancias amostrais, $\overline{\mathbf{Z}}$ e $\mathrm{S}_{Z}$, não dependem da condição de identificabilidade do modelo. Esta abordagem não envolve diretamente a distribuição dos dados observados mas sim a estrutura de matrizde variâncias covariâncias. Para o estudo de estimação do parâmetro $\beta$, consideramos os estimadores usuais sob normalidade. As suposições que se assumem para a consistencia do parâmetro $\beta$ são o conhecimento de uma o mais variâncias.

\subsubsection{Estimação}

Apresentamos a derivação da variância assintótica dos estimadores propostos para o parâmetro $\beta$, considerando $\sigma_{u}^{2}$ conhecida ou a razão de variâncias $k_{e}=\frac{\sigma_{u}^{2}}{\sigma_{e}^{2}}$ conhecida.

Denotamos a seguir as componentes da matriz $\Sigma$,

$$
\sigma_{Y Y}^{2}=\beta^{2} \sigma_{x}^{2}+\sigma_{e}^{2}, \quad \sigma_{Y X}^{2}=\beta \sigma_{x}^{2} \text { e } \sigma_{X X}^{2}=\sigma_{x}^{2}+\sigma_{u}^{2},
$$

notação utilizada para simplificar as notações subseqüente. 
Proposição 3.6. Considerando um modelo estrutural assimétrico aditivo com variância constante, segundo (3.18), e $\sigma_{u}^{2}$ conhecida, o estimador consistente, sob normalidade, é $\widehat{\beta}_{1}=\frac{S_{X Y}}{S_{X X}-\sigma_{u}^{2}}$, com variância assintótica:

$$
\Omega=\frac{(3 \delta-1)}{\sigma_{x}^{4}} \beta^{2} \sigma_{u}^{4}+\frac{\delta}{\sigma_{x}^{4}} \sum_{j=1}^{m}\left(\kappa_{j}-3\right) t_{2 j}^{2}\left(t_{1 j}^{2}-\beta t_{2 j}\right)^{2} .
$$

Demonstração. De acordo com o Teorema 2.9, temos que,

$$
\sqrt{n}\left(f\left[\operatorname{vech}\left(\mathbf{S}_{Z}\right)\right]-f[\operatorname{vech}(\boldsymbol{\Sigma})]\right) \stackrel{d}{\rightarrow} N(0, \Omega)
$$
onde $\Omega=\Pi \Lambda \Pi^{T}$, com $\Lambda$ em (3.23) e $\Pi=\frac{\partial f}{\partial v e c h^{T}(\boldsymbol{\Sigma})}$. Como, $f\left((x, y, z)^{T}\right)=\frac{y}{z-\sigma_{u}^{2}}$, temos
que,

$$
\boldsymbol{\Pi}=\frac{\partial f}{\partial v \operatorname{ech}^{T}(\Sigma)}=\left(0, \frac{1}{\sigma_{x}^{2}},-\frac{\beta}{\sigma_{x}^{2}}\right)=\frac{1}{\sigma_{x}^{2}}(0,1,-\beta) .
$$

Então,

$$
\sqrt{n}\left(\widehat{\beta}_{1}-\beta\right) \stackrel{d}{\rightarrow} N(0, \Omega)
$$

onde,

$$
\begin{aligned}
\Omega & =\frac{1}{\sigma_{x}^{4}}\left(\lambda_{22}-2 \beta \lambda_{23}+\beta^{2} \lambda_{33}\right)= \\
& =\frac{1}{\sigma_{x}^{4}}\left[(3 \delta-1)\left(\sigma_{Y X}^{4}-\beta \sigma_{Y X}^{2} \sigma_{X X}^{2}+\beta^{2} \sigma_{X X}^{4}\right)+\delta \sum_{j=1}^{m}\left(\kappa_{j}-3\right)\left(t_{1 j}^{2} t_{2 j}^{2}-2 \beta t_{1 j} t_{2 j}^{3}+\beta^{2} t_{2 j}^{4}\right)\right]= \\
& =\frac{(3 \delta-1)}{\sigma_{x}^{4}}\left(\sigma_{Y X}^{2}-\beta \sigma_{X X}^{2}\right)^{2}+\frac{\delta}{\sigma_{x}^{4}} \sum_{j=1}^{m}\left(\kappa_{j}-3\right) t_{2 j}^{2}\left(t_{1 j}^{2}-\beta t_{2 j}\right)^{2}= \\
& =\frac{(3 \delta-1)}{\sigma_{x}^{4}} \beta^{2} \sigma_{u}^{4}+\frac{\delta}{\sigma_{x}^{4}} \sum_{j=1}^{m}\left(\kappa_{j}-3\right) t_{2 j}^{2}\left(t_{1 j}^{2}-\beta t_{2 j}\right)^{2} .
\end{aligned}
$$

ficando assim provada a proposição.

Corolario 3.7. Considerando distribuição pseudo-elíptica para caracterizar o modelo assimétrico, temos que, $\Omega=\Omega_{E}$, onde

$$
\Omega_{E}=\beta^{2}(3 \delta-1) \frac{\sigma_{u}^{4}}{\sigma_{x}^{4}}
$$

No caso de considerar distribuição pseudo-normal, $\Omega=\Omega_{N}$, e temos que,

$$
\Omega_{N}=2 \beta^{2} \frac{\sigma_{u}^{4}}{\sigma_{x}^{4}}
$$

Ozán, N. S. 
Proposição 3.8. No modelo estrutural assimétrico aditivo con variância constante, segundo (3.18), com $k_{e}=\frac{\sigma_{e}^{2}}{\sigma_{u}^{2}}$ conhecida, o estimador consistente proposto para o parâmetro $\beta$, é

$$
\widehat{\beta}_{2}=\frac{S_{Y Y}-k_{e} S_{X X}-\left[\left(S_{Y Y}-k_{e} S_{X X}\right)^{2}+4 S_{Y X}^{2}\right]^{\frac{1}{2}}}{2 S_{Y X}}
$$

com variância assintótica,

$$
\Omega=(3 \delta-1)\left(a \sigma_{Y Y}^{2}+b \sigma_{Y X}^{2}+c \sigma_{X X}^{2}\right)^{2}+\delta \sum_{j=1}^{m}\left(\kappa_{j}-3\right)\left(a t_{1 j}^{2}+b t_{1 j} t_{2 j}+c t_{2 j}^{2}\right)^{2}
$$

onde, $\sigma_{Y Y}^{2}, \sigma_{Y X}^{2}$ e $\sigma_{X X}^{2}$ componentes da matriz $\Sigma e$

$$
\begin{aligned}
a & =\frac{1+\left(\sigma_{Y Y}^{2}-k_{e} \sigma_{X X}^{2}\right)\left[\left(\sigma_{Y Y}^{2}-k_{e} \sigma_{X X}^{2}\right)^{2}+4 \sigma_{Y X}^{4}\right]^{-\frac{1}{2}}}{2 \sigma_{Y X}^{2}}, \\
b & =\frac{-\sigma_{Y Y}^{2}+k_{e} \sigma_{X X}^{2}-\left[\left(\sigma_{Y Y}^{2}-k_{e} \sigma_{X X}^{2}\right)^{2}+4 \sigma_{Y X}^{4}\right]^{-\frac{1}{2}}}{2 \sigma_{Y X}^{2}}+\left[\left(\sigma_{Y Y}^{2}-k_{e} \sigma_{X X}^{2}\right)^{2}+4 \sigma_{Y X}^{4}\right]^{\frac{1}{2}}, \\
c & =\frac{-k_{e}+\left(\sigma_{Y Y}^{2}-k_{e} \sigma_{X X}^{2}\right) k_{e}\left[\left(\sigma_{Y Y}^{2}-k_{e} \sigma_{X X}^{2}\right)^{2}+4 \sigma_{Y X}^{4}\right]^{-\frac{1}{2}}}{2 \sigma_{Y X}^{2}} .
\end{aligned}
$$

Demonstração. Usando o Teorema 2.9, temos que,

$$
\sqrt{n}\left(f\left[\operatorname{vech}\left(\mathbf{S}_{Z}\right)\right]-f[\operatorname{vech}(\Sigma)]\right) \stackrel{d}{\rightarrow} N(0, \Omega)
$$

onde $\Omega=\Pi \Lambda \Pi^{\prime T}$, com $\Lambda$ em (3.23) e $\Pi=\frac{\partial f}{\partial v e c h^{T}(\Sigma)}$. Como $f\left((x, y, z)^{T}\right)=\frac{x-k_{e} z+\left[\left(x k_{e} z\right)^{2}+4 y^{2}\right]^{\frac{1}{2}}}{2 y}$, temos que, $\Pi=\frac{\partial f}{\partial v e c h^{T}(\Sigma)}=(a, b, c)$, com,

$$
\begin{aligned}
& a=\frac{\partial f}{\partial \sigma_{Y Y}^{2}}=\frac{1+\left(\sigma_{Y Y}^{2}-k_{e} \sigma_{X X}^{2}\right)\left[\left(\sigma_{Y Y}^{2}-k_{e} \sigma_{X X}^{2}\right)^{2}+4 \sigma_{Y X}^{4}\right]^{-\frac{1}{2}}}{2 \sigma_{Y X}^{2}}, \\
& b=\frac{\partial f}{\partial \sigma_{Y X}^{2}}=\frac{-\sigma_{Y Y}^{2}+k_{e} \sigma_{X X}^{2}-\left[\left(\sigma_{Y Y}^{2}-k_{e} \sigma_{X X}\right)^{4}+4 \sigma_{Y X}^{4}\right]^{-\frac{1}{2}}}{2 \sigma_{Y X}^{4}}+\left[\left(\sigma_{Y Y}^{2}-k_{e} \sigma_{X X}^{2}\right)^{2}+4 \sigma_{Y X}^{4}\right]^{\frac{1}{2}} \\
& c=\frac{\partial f}{\partial \sigma_{X X}^{2}}=\frac{-k_{e}+\left(\sigma_{Y Y}^{2}-k_{e} \sigma_{X X}^{2}\right) k_{e}\left[\left(\sigma_{Y Y}^{2}-k_{e} \sigma_{X X}^{2}\right)^{2}+4 \sigma_{Y X}^{4}\right]^{-\frac{1}{2}}}{2 \sigma_{Y X}^{2}}
\end{aligned}
$$

Logo,

$$
\sqrt{n}\left(\widehat{\beta}_{2}-\beta\right) \stackrel{d}{\rightarrow} N(0, \Omega)
$$

Ozán, N. S. 
com,

$$
\begin{aligned}
\Omega= & a^{2} \lambda_{11}+2 a b \lambda_{12}+b^{2} \lambda_{22}+2 a c \lambda_{13}+2 b c \lambda_{23}+c^{2} \lambda_{33}= \\
= & a^{2}\left[(3 \delta=1) \sigma_{Y Y}^{4}+\delta \sum_{j=1}^{m}\left(\kappa_{j}-3\right) t_{1 j}^{4}\right]+2 a b\left[(3 \delta-1) \sigma_{Y Y}^{2} \sigma_{Y X}^{2}+\delta \sum_{j=1}^{m}\left(\kappa_{j}-3\right) t_{1 j}^{3} t_{2 j}\right]+ \\
& +b^{2}\left[(3 \delta-1) \sigma_{Y X}^{4}+\delta \sum_{j=1}^{m}\left(\kappa_{j}-3\right) t_{1 j}^{2} t_{2 j}^{2}\right]+2 a c\left[(3 \delta-1) \sigma_{Y Y}^{2} \sigma_{X X}^{2}+\delta \sum_{j=1}^{m}\left(\kappa_{j}-3\right) t_{1 j}^{2} t_{2 j}^{2}\right]+ \\
& +2 b c\left[(3 \delta-1) \sigma_{Y x}^{2} \sigma_{X X}^{2}+\delta \sum_{j=1}^{m}\left(\kappa_{j}-3\right) t_{1 j}^{2} t_{2 j}^{2}\right]+c^{2}\left[(3 \delta-1) \sigma_{X X}^{4}+\delta \sum_{j=1}^{m}\left(\kappa_{j}-3\right) t_{2 j}^{4}\right]= \\
= & (3 \delta-1)\left(a \sigma_{Y Y}^{2}+b \sigma_{Y X}^{2}+c \sigma_{X X}\right)^{2}+\delta \sum_{j=1}^{m}\left(\kappa_{j}-3\right)\left(a t_{1 j}^{2}+b t_{1 j} t_{2 j}+c t_{2 j}^{2}\right)^{2},
\end{aligned}
$$

tal que, $\sigma_{Y Y}^{2}, \sigma_{Y X}^{2}$ e $\sigma_{X X}^{2}$ são as componentes da matriz $\Sigma$, obtendo assim a expressão (3.30).

Corolario 3.9. Considerando a distribuição pseudo-elíptica, temos que $\Omega=\Omega_{E}$, onde,

$$
\Omega_{E}=(3 \delta-1)\left(a \sigma_{Y Y}^{2}+b \sigma_{Y X}+c \sigma_{X X}^{2}\right)^{2}
$$

No caso de distribuição pseudo-normal, temos que $\Omega=\Omega_{N}$, e

$$
\Omega_{N}=2\left(a \sigma_{Y Y}^{2}+b \sigma_{Y X}^{2}+c \sigma_{X X}^{2}\right)^{2}
$$

No final do capítulo apresentamos um resumo dos resultados obtidos para as respectivas variâncias assintóticas dos estimadores para o parâmetro $\beta$.

\subsection{Modelo Estrutural Assimétrico Linear Multiplica- tivo}

\subsubsection{Especificação do Modelo}

O modelo estrutural lineal multiplicativo com erro nas variáveis, como definido em (1.2), pode ser escrito como

$$
\mathrm{Z}_{i}=\left(\begin{array}{c}
Y_{i} \\
X_{i}
\end{array}\right)=\left(\begin{array}{c}
\alpha+\mathbf{b}^{T} \mathbf{r}_{i} \\
\mathbf{r}_{i}^{T} \operatorname{Br}_{i}
\end{array}\right)
$$

$\operatorname{com} \mathbf{r}_{i}=\left(x_{i}, e_{i}, u_{i}\right)^{T}, \mathrm{~b}=(\beta, 1,0)^{T} \mathrm{e}$

$$
\mathrm{B}=\frac{1}{2}\left(\begin{array}{lll}
0 & 0 & 1 \\
0 & 0 & 0 \\
1 & 0 & 0
\end{array}\right),
$$

Ozán, N. S. 
com, $\left(Y_{i}, X_{i}\right)^{T}$ variáveis observadas, sendo $e_{i} \sim\left(0, \sigma_{e_{i}}^{2}\right)$ e $u_{i} \sim\left(1, \sigma_{u_{i}}^{2}\right)$ os erros aleatórios do modelo, e $x_{i} \sim\left(\mu_{x}, \sigma_{x_{i}}^{2}\right)$, para $i=1, \ldots, n$, independentes.

Assumimos que,

- $\mathbf{r}_{i}-\eta_{i}=\left(x_{i}-\mu_{x}, e_{i}, u_{i}-1\right)^{T}$ tem distribuição assimétrica (Definição 2.1), com média zero, matriz de covariância $\Psi_{i}=\operatorname{diag}\left\{\sigma_{x_{i}}^{2}, \sigma_{e_{i}}^{2}, \sigma_{u_{i}}^{2}\right\}$, assimetria $\lambda_{i}$ e e curtose $\kappa_{i}, \operatorname{com} E\left[\mathbf{r}_{i}\right]=\left(\mu_{x}, 0,1\right)^{T}$ e $\operatorname{Var}\left[\mathbf{r}_{i}\right]=\Psi_{i}, i=1, \ldots, n$.

Usando a Definição 2.1 , e considerando $\mathbf{r}_{i}^{*}=\mathbf{r}_{i}-\boldsymbol{\eta}$, temos que,

$$
\mathbf{r}_{i}^{*}=\mathbf{r}_{i}-\boldsymbol{\eta}=\left(x_{i}-\mu_{x}, e_{i}, u_{i}-1\right)^{T}=v_{i} \mathbf{A}_{i} \boldsymbol{\xi}_{i}, \quad i=1, \ldots, n,
$$

onde,

- $\boldsymbol{\xi}_{i}=\left(\xi_{1 i}, \xi_{2 i}, \ldots, \xi_{m i}\right)^{\prime}$, tal que $\xi_{1 i}, \xi_{2 i}, \ldots, \xi_{m i}$ são variáveis aleatórias independentes $\operatorname{com} E\left[\xi_{j i}\right]=0, E\left[\xi_{j i}^{2}\right]=1, E\left[\xi_{j i}^{3}\right]=\zeta_{j}$ e $E\left[\xi_{j i}^{4}\right]=\kappa_{j}, j=1, \ldots, m$,

- $v_{i}$ variável aleatória independente de $\boldsymbol{\xi}_{i} \operatorname{com} E\left[v_{i}^{2}\right]=1, E\left[v_{i}^{3}\right]=\gamma$ e $E\left[v_{i}^{4}\right]=\delta$, $i=1, \ldots, n, \mathrm{e}$

- $\mathbf{A}_{i}=\left(a_{i_{l j}}\right)$, matriz de dimensão $3 \times m$, com posto igual a 3 , tal que $\mathbf{A}_{i} \mathbf{A}_{i}^{\prime}=\Psi_{i}$, $i=1, \ldots, n, l=1,2,3$ e $j=1, \ldots, m$.

Similarmente a obtenção de (3.3), o coeficiente de assimetria e cutose marginal, respectivamente, são dados por

$$
\begin{gathered}
\lambda_{1 i}=\lambda_{i}\left(x_{i}-\mu_{x}\right)=\gamma \frac{\sum_{j=1}^{m} a_{i_{1 j}}^{3} \zeta_{j}}{\left(\sum_{j=1}^{m} a_{i_{1 j}}^{2}\right)^{3}}, \\
\lambda_{2 i}=\lambda_{i}\left(e_{i}\right)=\gamma \frac{\sum_{j=1}^{m} a_{i_{2 j}}^{3} \zeta_{j}}{\left(\sum_{j=1}^{m} a_{i_{2 j}}^{2}\right)^{3}}, \quad \kappa_{1 i}=\kappa_{i}\left(x_{i}-\mu_{x}\right)=\delta\left\{\sum_{j=1}^{m} \frac{a_{i_{1 j}}^{4}\left(\kappa_{i}-3\right)}{\sigma_{x_{i}}^{2}}+3\right\}, \\
\lambda_{3 i}=\lambda_{i}\left(u_{i}-1\right)=\gamma \frac{\sum_{j=1}^{m} a_{i_{3 j}}^{3} \zeta_{j}}{\left(\sum_{j=1}^{m} a_{i_{3 j}}^{2}\right)^{3}}, \quad \kappa_{3 i}=\kappa_{i}\left(u_{i}\right)=\delta\left\{\sum_{j=1}^{m} \frac{a_{i_{2 j}}^{4}\left(\kappa_{i}-3\right)}{\sigma_{e_{i}}^{2}}+3\right\},
\end{gathered}
$$

Portanto, segundo (2.3), temos que,

$$
\mathrm{r}^{*}=\mathrm{r}_{i}-\eta=\left(x_{i}-\mu_{x}, e_{i}, u_{i}\right)^{T} \sim S\left(0, \Psi_{i}, \lambda, \kappa\right),
$$


$\operatorname{com} \lambda=\left(\lambda_{1 i}, \lambda_{2 i}, \lambda_{3 i}\right)^{T}$ e $\kappa=\left(\kappa_{1 i}, \kappa_{2 i}, \kappa_{3 i}\right)^{T}, i=1, \ldots, n$

Notamos que analogamente ao modelo aditivo, neste caso, também verifica-se a Propriedade 1 . Além disso, como $\mathbf{r}_{i}^{*}=\mathbf{r}_{i}-\boldsymbol{\eta}=v_{i} \mathbf{A}_{i} \boldsymbol{\xi}_{i}$, então,

$$
\mathbf{r}_{i}^{*}=\left(\begin{array}{c}
x_{i}-\mu_{x} \\
e_{i} \\
u_{i}-1
\end{array}\right)=\left(\begin{array}{c}
v_{i} \sum_{j=1}^{m} a_{i_{1 j}} \xi_{j i} \\
v_{i} \sum_{j=1}^{m} a_{i_{2 j}} \xi_{j i} \\
v_{i} \sum_{j=1}^{m} a_{i_{3 j}} \xi_{j i}
\end{array}\right)
$$

Portanto,

$$
x_{i}-\mu_{x}=v_{i} \sum_{j=1}^{m} a_{i_{1 j}} \xi_{j i}, \quad e_{i}=v_{i} \sum_{j=1}^{m} a_{i_{2 j}} \xi_{j i}, \quad u_{i}-1=v_{i} \sum_{j=1}^{m} a_{i_{3 j}} \xi_{j i} .
$$

Observamos que os momentos de $\mathbf{r}_{i}^{*}$ são aqueles obtidos na Proposição 3.1.

Seja $\mathbf{Z}_{1}, \mathbf{Z}_{2}, \ldots$ seqüência de vetores aleatórios independentes, onde $\mathbf{Z}_{i}$ é definida em (3.33) e distribuição assimétrica com parâmetro de locação e escala, de modo que,

$$
\boldsymbol{\mu}=\left(\begin{array}{c}
\alpha+\beta \mu_{x} \\
\mu_{x}
\end{array}\right) \text { e } \quad \Sigma_{i}=\left(\begin{array}{cc}
\beta^{2} \sigma_{x_{i}}^{2}+\sigma_{e_{i}}^{2} & \beta \sigma_{x_{i}}^{2} \\
\beta \sigma_{x_{i}}^{2} & \sigma_{x_{i}}^{2} \sigma_{u_{i}}^{2}+\sigma_{x_{i}}^{2}+\mu_{x_{i}}^{2} \sigma_{u_{i}}^{2}
\end{array}\right) .
$$

Considerando agora $\epsilon_{i}=\mathrm{Z}_{i}-\boldsymbol{\mu}$, temos que $\epsilon_{i}$ tem distribuição assimétrica com parâmetro locação e escala, 0 e $\Sigma_{i}$, respectivamente, $i-1, \ldots, n$. Assim, pela Definição 2.1, temos que,

$$
\epsilon_{i}=v_{i} \mathbf{T}_{i} \xi_{i}
$$

onde,

- $\xi_{i}=\left(\xi_{1 i}, \xi_{2 i}, \ldots, \xi_{m i}\right)^{T}$ e $v$ são variáveis aleatórias independentes que verificam as mesmas condições dadas em (3.13).

- $\mathbf{T}_{\mathbf{i}}=\left(t_{i_{p j}}\right)$ matriz de dimensão $2 \times m$, com posto 2 , tal que $\mathbf{T}_{i} \mathbf{T}_{i}^{T}=\Sigma_{i}, p=1,2$, $j=1, \ldots, m$ e $i=1, \ldots, n$, Portanto,

$$
\mathbf{T}_{i}=\left(\begin{array}{cccc}
t_{i_{11}} & t_{i_{12}} & \ldots & t_{i_{1 m}} \\
t_{i_{21}} & t_{i_{22}} & \ldots & t_{i_{2 m}}
\end{array}\right)
$$

Definimos $t_{j}$ como a j-ésima coluna de $\boldsymbol{T}_{i}$, para $j=1, \ldots, m$ e $i=1, \ldots, n$. 
Portanto, para $i=1, \ldots, n$,

$$
\Sigma_{i}=\mathbf{T}_{i} \mathbf{T}_{i}^{T}=\left(\begin{array}{cc}
\sum_{j=1}^{m} t_{i_{1 j}}^{2} & \sum_{j=1}^{m} t_{i_{1 j}} t_{i_{2 j}} \\
\sum_{j=1}^{m} t_{i_{1 j}} t_{i_{2 j}} & \sum_{j=1}^{m} t_{i_{2 j}}^{2}
\end{array}\right)
$$

e pela expressão (3.36), temos que,

$$
\beta^{2} \sigma_{x_{i}}^{2}+\sigma_{e_{i}}^{2}=\sum_{j=1}^{m} t_{i_{1 j}}^{2}, \quad \beta \sigma_{x_{i}}^{2}=\sum_{j=1}^{m} t_{i_{1 j}} t_{i_{2 j}}, \quad \sigma_{x_{i}}^{2} \sigma_{u_{i}}^{2}+\sigma_{x_{i}}^{2}+\mu_{x_{i}}^{2} \sigma_{u_{i}}^{2}=\sum_{j=1}^{m} t_{i_{2 j}}^{2} .
$$

Além disso, como $\epsilon_{i}=\mathrm{Z}_{i}-\boldsymbol{\mu}$, escrevemos,

$$
\epsilon_{i}=\left(\begin{array}{c}
\beta\left(x_{i}-\mu_{x}\right)+e_{i} \\
x_{i} u_{i}-\mu_{x}
\end{array}\right)
$$

então temos que,

$$
\epsilon_{i}=\left(\begin{array}{c}
\beta\left(x_{i}-\mu_{x}\right)+e_{i} \\
x_{i} u_{i}-\mu_{x_{i}}
\end{array}\right)=v_{i} \mathbf{T}_{i} \xi_{i}=\left(\begin{array}{c}
v_{i} \sum_{j=1}^{m} t_{i_{1 j}} \xi_{j i} \\
v_{i} \sum_{j=1}^{m} t_{i_{2 j}} \xi_{j i}
\end{array}\right)
$$

O parâmetro de assimetria e curtose marginal são, respectivamente,

$$
\begin{gathered}
\lambda_{1 i}^{*}=\lambda_{i}\left(\beta\left(x_{i}-\mu_{x}\right)+e_{i}\right)=\gamma \sum_{j=1}^{m} \frac{t_{i_{1 j}}^{3} \zeta_{j}}{\sigma_{i i}^{\frac{3}{2}}}, \quad \lambda_{2 i}^{*}=\lambda_{i}\left(x_{i} u_{i}-\mu_{x}\right)=\gamma \sum_{j=1}^{m} \frac{t_{i_{2 j}}^{3} \zeta_{j}}{\sigma_{i i}^{\frac{3}{2}}}, \\
\kappa_{1 i}^{*}=\kappa_{i}\left(\beta\left(x_{i}-\mu_{x}\right)+e_{i}\right)=\delta\left\{\sum_{j=1}^{m} \frac{i_{i_{1 j}}^{4}\left(\kappa_{i}-3\right)}{\sigma_{i i}^{2}}+3\right\}, \\
\kappa_{2 i}^{*}=\kappa_{i}\left(x_{i} u_{i}-\mu_{x}\right)=\delta\left\{\sum_{j=1}^{m} \frac{t_{i_{2 j}}^{4}\left(\kappa_{i}-3\right)}{\sigma_{i i}^{2}}+3\right\} .
\end{gathered}
$$

Portanto, se $\epsilon_{i}=v_{i} \mathrm{~T}_{i} \xi_{i}$, segundo a equação (3.37), temos que, $\epsilon_{i} \stackrel{i i d}{\sim} S\left(0, \Sigma_{i}, \lambda_{i}^{*}, \kappa_{i}^{*}\right)$, onde $\lambda_{i}^{*}=\left(\lambda_{1 i}^{*}, \lambda_{2 i}^{*}\right)^{T}$ e $\kappa_{i}^{*}=\left(\kappa_{1 i}^{*}, \kappa_{2 i}^{*}\right)$ cujas expressões são dadas em (3.40) e (3.41), respectivamente, $i=1, \ldots, n$.

A seguir, analisamos um modelo multiplicativo com variância constante e derivamos as propriedades assintóticas de $\overline{\mathbf{Z}}$ e $\mathrm{S}_{Z}$. 


\subsubsection{Modelo Estrutural Assimétrico Linear Multiplicativo com variância constante}

Nesta seção consideramos o modelo multiplicativo estrutural assimétrico com variância constante, ou seja $\Psi_{i}=\Psi$, ou equivalentemente $\mathrm{A}_{i}=\mathrm{A}$ de dimensão $3 \times m$, com $\mathrm{AA}^{T}=\Psi, \mathrm{e}$

$$
\mathbf{r}_{i}-\boldsymbol{\eta}=\left(x_{i}-\mu_{x}, e_{i}, u_{i}\right)^{\prime}=v_{i} \mathbf{A} \boldsymbol{\xi}_{i}, \quad i=1, \ldots, n .
$$

Portanto,

$$
\left(\begin{array}{c}
x_{i}-\mu_{x} \\
e_{i} \\
u_{i}-1
\end{array}\right)=\left(\begin{array}{c}
v_{i} \sum_{j=1}^{m} a_{1 j} \xi_{j i} \\
v_{i} \sum_{\substack{j=1 \\
m}}^{m} a_{2 j} \xi_{j i} \\
v_{i} \sum_{j=1}^{m} a_{3 j} \xi_{j i}
\end{array}\right) .
$$

Assim, em (3.37), temos que $\boldsymbol{\epsilon}_{i}=v_{i} \mathbf{T} \boldsymbol{\xi}_{i}$. Portanto, agora a expressão para $\boldsymbol{\Sigma}$ é,

$$
\boldsymbol{\Sigma}=\mathbf{T} \mathbf{T}^{T}=\left(\begin{array}{cc}
\sum_{j=1}^{m} t_{1 j}^{2} & \sum_{j=1}^{m} t_{1 j} t_{2 j} \\
\sum_{j=1}^{m} t_{1 j} t_{2 j} & \sum_{j=1}^{m} t_{2 j}^{2}
\end{array}\right)=\left(\begin{array}{ll}
\sigma_{Y Y}^{2} & \sigma_{Y X}^{2} \\
\sigma_{Y X}^{2} & \sigma_{X X}^{2}
\end{array}\right),
$$

onde,

$$
\sigma_{Y Y}^{2}=\beta^{2} \sigma_{x}^{2}+\sigma_{e}^{2}, \quad \sigma_{Y X}^{2}=\beta \sigma_{x}^{2}, \quad \sigma_{X X}^{2}=\sigma_{x}^{2} \sigma_{u}^{2}+\sigma_{x}^{2}+\mu_{x}^{2} \sigma_{u}^{2}
$$

A seguir, apresentamos resultados utilizados para o estudo assintótico de $\overline{\mathbf{Z}}$ e $\mathbf{S}_{Z}$.

Lema 3.10. Seja $\epsilon_{i}=v_{i} \mathbf{T} \xi_{i}$, então, $\Gamma=E\left[\epsilon_{i} v e c \operatorname{vec}^{T}\left(\epsilon_{i} \epsilon_{i}^{T}\right)\right], i=1, \ldots, n$, é dada por,

$$
\Gamma=\gamma \sum_{j=1}^{m} \mathbf{T}_{1 j} \zeta_{j}
$$

$\operatorname{com} \gamma=E\left(v_{i}^{3}\right), \zeta_{j}=E\left(\xi_{j i}^{3}\right), j=1, \ldots, m, e$

$$
\mathbf{T}_{1 j}=\left(\begin{array}{ccc}
t_{1 j}^{3} & t_{1 j}^{2} t_{2 j} & t_{1 j} t_{2 j}^{2} \\
t_{1 j}^{2} t_{2 j} & t_{1 j} t_{2 j}^{2} & t_{2 j}^{3}
\end{array}\right)
$$

Ozán, N. S. 
Demonstração. Derivando vech $\left(\epsilon_{i} \epsilon_{i}^{T}\right)$ temos que,

$$
\operatorname{vech}\left(\epsilon_{i} \epsilon_{i}^{T}\right)=\left(\begin{array}{c}
{\left[\beta\left(x_{i}-\mu_{x}\right)+e_{i}\right]^{2}} \\
\left(\beta\left(x_{i}-\mu_{x}+e_{i}\right)\left(x_{i} u_{i}-\mu_{x}\right)\right. \\
\left(x_{i} u_{i}-\mu_{x}\right)^{2}
\end{array}\right),
$$

e definindo os elementos do produto $\epsilon_{1} \operatorname{vech}^{T}\left(\epsilon_{i} \epsilon_{i}^{T}\right)$ como,

$$
\epsilon_{i} \operatorname{vech}^{T}\left(\epsilon_{i} \epsilon_{i}^{T}\right)=\left(\begin{array}{lll}
E_{11} & E_{12} & E_{13} \\
E_{21} & E_{22} & E_{23}
\end{array}\right)
$$

onde,

$$
\begin{array}{ll}
E_{11}=\left[\beta\left(x_{i}-\mu_{x}\right)+e_{i}\right]^{3} & E_{21}=\left[\beta\left(x_{i}-\mu_{x}\right)+e_{i}\right]^{2}\left(x_{i} u_{i}-\mu_{x}\right) \\
E_{12}=\left[\beta\left(x_{i}-\mu_{x}\right)+e_{i}\right]^{2}\left(x_{i} u_{i}-\mu_{x}\right) & E_{22}=\left[\beta\left(x_{i}-\mu_{x}\right)+e_{i}\right]\left(x_{i} u_{i}-\mu_{x}\right)^{2} \\
E_{13}=\left[\beta\left(x_{i}-\mu_{x}\right)+e_{i}\right]\left(x_{i} u_{i}-\mu_{x}\right)^{2} & E_{23}=\left[x_{i} u_{i}-\mu_{x}\right]^{3} .
\end{array}
$$

Logo, $\boldsymbol{\Gamma}=E\left[\epsilon_{i} \operatorname{vech}^{T}\left(\epsilon_{i} \epsilon_{i}^{T}\right)\right]$, então $\Gamma=E\left[E_{p q}\right], p=1,2$, e $q=1,2,3$.

Por outro lado, $\epsilon_{i}$ pode ser escrito como $\epsilon_{i}=\left(\begin{array}{c}v_{i} \sum_{j=1}^{m} t_{1 j} \xi_{j i} \\ v_{i} \sum_{j=1}^{m} t_{2 j} \xi_{j i}\end{array}\right)$, de onde obtemos os seguintes valores esperados,

$$
\begin{gathered}
E\left\{\left[\beta\left(x_{i}-\mu_{x}\right)+e_{i}\right]^{3}\right\}=E\left\{\left[v_{i} \sum_{j=1}^{m} t_{1 j} \xi_{j}\right]^{3}\right\}=\gamma \sum_{j=1}^{m} t_{1 j}^{3} \zeta_{j}, \\
E\left\{\left[x_{i} u_{i}-\mu_{x}\right]^{3}\right\}=E\left\{\left[v_{i} \sum_{j=1}^{m} t_{2 j} \xi_{j}\right]^{3}\right\}=\gamma \sum_{j=1}^{m} t_{2 j}^{3} \zeta_{j}, \\
E\left\{\left[\beta\left(x_{i}-\mu_{x}\right)+e_{i}\right]^{2}\left[x_{i} u_{i}-\mu_{x}\right]\right\}=E\left\{\left[v_{i} \sum_{j=1}^{m} t_{1 j} \xi_{j}\right]^{2}\left[v_{i} \sum_{j=1}^{m} t_{2 j} \xi_{j}\right]\right\}=\gamma \sum_{j=1}^{m} t_{1 j}^{2} t_{2 j} \zeta_{j}, \\
\left.E\left\{\left[\beta\left(x_{i}-\mu_{x}\right)+e_{i}\right]\left[x_{i} u_{i}-\mu_{x}\right]^{2}\right]\right\}=E\left\{\left[v_{i} \sum_{j=1}^{m} t_{1 j} \xi_{j}\right]\left[v \sum_{j=1}^{m} t_{2 j} \xi_{j}\right]^{2}\right\}=\gamma \sum_{j=1}^{m} t_{1 j} t_{2 j}^{2} \zeta_{j} .
\end{gathered}
$$

Portanto,

$$
\boldsymbol{\Gamma}=\gamma\left(\begin{array}{ccc}
\sum_{j=1}^{m} t_{1 j}^{3} \zeta_{j} & \sum_{j=1}^{m} t_{1 j}^{2} t_{2 j} \zeta_{j} & \sum_{j=1}^{m} t_{1 j} t_{2 j}^{2} \zeta_{j} \\
\sum_{j=1}^{m} t_{1 j}^{2} t_{2 j} \zeta_{j} & \sum_{j=1}^{m} t_{1 j} t_{2 j}^{2} \zeta_{j} & \sum_{j=1}^{m} t_{2 j}^{3} \zeta_{j}
\end{array}\right)=\gamma \sum_{j=1}^{m} \mathrm{~T}_{1 j} \zeta_{j},
$$

com $\mathbf{T}_{1 j}$ como em (3.45), o que prova o lema.

Ozán, N. S. 
Lema 3.11. Seja $\epsilon_{i}=v_{i} \mathbf{T} \xi_{i}, i=1, \ldots, n$, então, $\Lambda=\operatorname{Var}\left[\operatorname{vech}\left(\epsilon_{i} \epsilon_{i}^{T}\right)\right]$ é dada por,

$$
\Lambda=(3-\delta) \mathbf{T}^{*}+\delta \sum_{j=1}^{m}\left(\kappa_{j}-3\right) \mathbf{T}_{2 j}
$$

com,

$$
\begin{gathered}
\mathbf{T}^{*}=\left(\begin{array}{ccc}
\left(\sum_{j=1}^{m} t_{1 j}^{2}\right)^{2} & \left(\sum_{j=1}^{m} t_{1 j}^{2}\right)\left(\sum_{j=1}^{m} t_{1 j} t_{2 j}\right) & \left(\sum_{j=1}^{m} t_{1 j}^{2}\right)\left(\sum_{j=1}^{m} t_{2 j}^{2}\right) \\
\ldots & \left(\sum_{j=1}^{m} t_{1 j} t_{2 j}\right)^{2} & \left(\sum_{j=1}^{m} t_{1 j} t_{2 j}\right)\left(\sum_{j=1}^{m} t_{2 j}^{2}\right) \\
\ldots & \ldots & \left(\sum_{j=1}^{m} t_{2 j}^{2}\right)^{2}
\end{array}\right), \\
\vdots \\
\mathbf{T}_{2 j}=\left(\begin{array}{ccc}
t_{1 j}^{4} & t_{1 t_{2 j}^{3}}^{3} & t_{1 j}^{2} t_{2 j}^{2} \\
\cdots & t_{1 j}^{2} t_{2 j}^{2} & t_{1 j} t_{2 j}^{3} \\
\ldots & \cdots & t_{2 j}^{4}
\end{array}\right) .
\end{gathered}
$$

Demonstração. A demonstração do lema em (3.46) é análoga à feita para o modelo aditivo em (3.23). A diferença entre ambas expressões é a matriz $\mathbf{T}^{*}$ e $\mathbf{T}_{2 j}$, já que a estrutura de covariância dos modelos é diferente em cada caso.

Observação 6. Sendo $\Lambda=\left(\lambda_{p q}\right), p, q=1,2,3$, que de acordo com (3.46), podemos escrever,

$$
\begin{aligned}
& \lambda_{11}=(3 \delta-1)\left(\beta^{2} \sigma_{x}^{2}+\sigma_{e}^{2}\right)^{2}+\delta \sum_{j=1}^{m}\left(\kappa_{j}-3\right) t_{1 j}^{4} \\
& \lambda_{12}=(5 \delta-1)\left(\beta^{2} \sigma_{x}^{2}+\sigma_{e}^{2}\right) \beta \sigma_{x}^{2}+\delta \sum_{j=1}^{m}\left(\kappa_{j}-3\right) t_{1 j}^{3} t_{2 j} \\
& \lambda_{13}=(3 \delta-1)\left(\beta^{2} \sigma_{x}^{2}+\sigma_{e}^{2}\right)\left(\sigma_{x}^{2} \sigma_{u}^{2}+\sigma_{x}^{2}+\mu_{x}^{2} \sigma_{u}^{2}\right)+\delta \sum_{j=1}^{m}\left(\kappa_{j}-3\right) t_{1 j}^{2} t_{2 j}^{2} \\
& \lambda_{22}=(9 \delta-1) \beta^{2} \sigma_{x}^{4}+\delta \sum_{j=1}^{m}\left(\kappa_{j}-3\right) t_{1 j}^{2} t_{2 j}^{2} \\
& \lambda_{23}=(5 \delta-1) \beta \sigma_{x}^{2}\left(\sigma_{x}^{2} \sigma_{u}^{2}+\sigma_{x}^{2}+\mu_{x}^{2} \sigma_{u}^{2}\right)+\delta \sum_{j=1}^{m}\left(\kappa_{j}-3\right) t_{1 j} t_{2 j}^{3} \\
& \lambda_{33}=(3 \delta-1)\left(\sigma_{x}^{2} \sigma_{u}^{2}+\sigma_{x}^{2}+\mu_{x}^{2} \sigma_{u}^{2}\right)^{2}+\delta \sum_{j=1}^{m}\left(\kappa_{j}-3\right) t_{2 j}^{4},
\end{aligned}
$$

Ozán, N. S. 
e portanto,

$$
\begin{aligned}
\Lambda= & (3-\delta)\left(\begin{array}{ccc}
\left(\beta^{2} \sigma_{x}^{2}+\sigma_{e}^{2}\right)^{2} & \left(\beta^{2} \sigma_{x}^{2}+\sigma_{e}^{2}\right) \beta \sigma_{x}^{2} & \left(\beta^{2} \sigma_{x}^{2}+\sigma_{e}^{2}\right)\left(\sigma_{x}^{2} \sigma_{u}^{2}+\sigma_{x}^{2}+\mu_{x}^{2} \sigma_{u}^{2}\right) \\
\cdots & \beta^{2} \sigma_{x}^{4} & \beta^{2} \sigma_{x}^{4}\left(\sigma_{x}^{2} \sigma_{u}^{2}+\sigma_{x}^{2}+\mu_{x}^{2} \sigma_{u}^{2}\right) \\
\cdots & \cdots & \left(\sigma_{x}^{2} \sigma_{u}^{2}+\sigma_{x}^{2}+\mu_{x}^{2} \sigma_{u}^{2}\right)^{2}
\end{array}\right)+ \\
& +\delta \sum_{j=1}^{m}\left(\kappa_{j}-3\right) \mathbf{T}_{2 j},
\end{aligned}
$$

onde $\mathbf{T}_{2 j}$ definido no lema anterior.

Corolario 3.12. 1. Se $\epsilon_{i}$ tem distribuição pseudo-elíptica, isto é, $\kappa_{j}=3$, para todo $j=1, \ldots, m$, definimos $\Lambda=\Lambda_{E}$ que é dada por,

$$
\Lambda_{E}=(3-\delta)\left(\begin{array}{ccc}
\left(\beta^{2} \sigma_{x}^{2}+\sigma_{e}^{2}\right)^{2} & \left(\beta^{2} \sigma_{x}^{2}+\sigma_{e}^{2}\right) \beta \sigma_{x}^{2} & \left(\beta^{2} \sigma_{x}^{2}+\sigma_{e}^{2}\right)\left(\sigma_{x}^{2} \sigma_{u}^{2}+\sigma_{x}^{2}+\mu_{x}^{2} \sigma_{u}^{2}\right) \\
\cdots & \beta^{2} \sigma_{x}^{4} & \beta^{2} \sigma_{x}^{4}\left(\sigma_{x}^{2} \sigma_{u}^{2}+\sigma_{x}^{2}+\mu_{x}^{2} \sigma_{u}^{2}\right) \\
\cdots & \cdots & \left(\sigma_{x}^{2} \sigma_{u}^{2}+\sigma_{x}^{2}+\mu_{x}^{2} \sigma_{u}^{2}\right)^{2}
\end{array}\right) .
$$

2. Se $\boldsymbol{\epsilon}_{i}$ tem distribuição pseudo-normal, isto é, $\kappa_{j}=3$, para todo $j=1, \ldots, m$, e $\delta=1$, definimos $\boldsymbol{\Lambda}=\boldsymbol{\Lambda}_{N}$ que é dada por,

$$
\Lambda_{N}=2\left(\begin{array}{ccc}
\left(\beta^{2} \sigma_{x}^{2}+\sigma_{e}^{2}\right)^{2} & \left(\beta^{2} \sigma_{x}^{2}+\sigma_{e}^{2}\right) \beta \sigma_{x}^{2} & \left(\beta^{2} \sigma_{x}^{2}+\sigma_{e}^{2}\right)\left(\sigma_{x}^{2} \sigma_{u}^{2}+\sigma_{x}^{2}+\mu_{x}^{2} \sigma_{u}^{2}\right) \\
\cdots & \beta^{2} \sigma_{x}^{4} & \beta^{2} \sigma_{x}^{4}\left(\sigma_{x}^{2} \sigma_{u}^{2}+\sigma_{x}^{2}+\mu_{x}^{2} \sigma_{u}^{2}\right) \\
\cdots & \ldots & \left(\sigma_{x}^{2} \sigma_{u}^{2}+\sigma_{x}^{2}+\mu_{x}^{2} \sigma_{u}^{2}\right)^{2}
\end{array}\right) .
$$

Logo, concluímos que,

1. $\epsilon_{i}$ tem distribuição assimétrica, ou seja, $\epsilon_{i} \stackrel{i i d}{\sim} S\left(0, \Sigma, \lambda^{*}, \kappa^{*}\right), i=1, \ldots, n$.

2. $\mathbf{r}_{i}^{*}=\mathbf{r}_{i}-\boldsymbol{\eta}$ tem momento de quarta ordem finito.

Portanto, $\Lambda=\operatorname{Var}\left[\operatorname{vech}\left(\epsilon_{1} \epsilon_{1}^{T}\right)\right]<\infty$.

3. Se $\overline{\mathbf{Z}}=\frac{1}{n} \sum_{i=1}^{n} \mathbf{Z}_{i}$ e $\mathbf{S}_{Z}=\frac{1}{n} \sum_{i=1}^{n}\left(\mathbf{Z}_{i}-\overline{\mathbf{Z}}\right)\left(\mathbf{Z}_{i}-\overline{\mathbf{Z}}\right)^{T}$, temos que, se verificam as suposições $A_{1}, A_{2}$ e $A_{3}$ do Lema 2.1 em Arellano-Valle, Bolfarine e Gasco (2002), portanto temos que, quando $n \rightarrow \infty$,

$$
\overline{\mathrm{Z}} \stackrel{q c}{\rightarrow} \mu \quad \text { e } \quad \mathrm{S}_{Z} \stackrel{q c}{\rightarrow} \Sigma .
$$

Portanto, analogamente ao realizado no modelo aditivo, podemos enunciar o seguinte resultado.

Teorema 3.13. Sob as condições mencionadas acima, temos que,

$$
\left(\begin{array}{c}
\sqrt{n}(\overline{\mathbf{Z}}-\mu) \\
\sqrt{n} \operatorname{vech}\left(\mathrm{S}_{Z}-\Sigma\right)
\end{array}\right) \stackrel{d}{\rightarrow} N_{5}\left(\left(\begin{array}{l}
0 \\
0
\end{array}\right) ;\left(\begin{array}{cc}
\Sigma & \Gamma \\
\Gamma^{T} & \Lambda
\end{array}\right)\right)
$$

onde, $\Sigma=\mathrm{TT}^{T}$, com $\Gamma$ e $\Lambda$ obtidos no Lema (3.10) e (3.11), respectivamente. 


\subsubsection{Estimação}

Abordamos a seguir o problema da estimação do parâmetro $\beta$. Utilizamos os estimadores propostos na literatura (Arellano Valle, Bolfarine e Gasco ,2002) para os modelos elípticos. Derivando a variância assintótica para os estimadores propostos temos os seguintes resultados.

Proposição 3.14. Considerando um modelo estrutural assimétrico multiplicativo con variância constante, com o parâmetro $\alpha$ conhecido, tal que $\alpha=0$, então o estimador consistente de $\beta$ é $\widehat{\beta}_{1}=\frac{\bar{Y}}{\bar{X}}$ (Arellano-Valle et al, 2002), com variância assintótica,

$$
\Omega=\frac{\sigma_{u}^{2}}{\mu_{x}^{2}}\left[k_{e}+\beta^{2}\left(\sigma_{u}^{2} k_{x}+\mu_{x}^{2}\right)\right]
$$

onde $k_{x}=\frac{\sigma_{x}^{2}}{\sigma_{u}^{2}}$ e $k_{e}=\frac{\sigma_{e}^{2}}{\sigma_{u}^{2}}$.

Demonstração. Aplicando o Teorema 2.9, temos que,

$$
\sqrt{n}(f(\overline{\mathbf{Z}}-\boldsymbol{\mu})) \stackrel{d}{\rightarrow} N(0, \Omega)
$$

onde $\Omega=\Pi \Sigma \Pi^{T}, \Sigma$ como em (3.43) e $\Pi=\frac{\partial f}{\partial \boldsymbol{\mu}}$. Note que se $f\left((x, y)^{T}\right)=\frac{x}{y}$, temos que, $\Pi=\frac{1}{\mu_{x}}(1,-\beta)$.

Então,

$$
\sqrt{n}\left(\widehat{\beta}_{1}-\beta\right) \stackrel{d}{\rightarrow} N(0, \Omega)
$$

e calculando $\Omega=\Pi \Sigma \Pi^{T}$, temos que,

$$
\begin{aligned}
\Omega & =\frac{1}{\mu_{x}^{2}}\left(\sigma_{Y Y}^{2}-2 \beta \sigma_{Y X}^{2}+\beta^{2} \sigma_{X X}^{2}\right)=\frac{1}{\mu_{x}^{2}}\left[\sigma_{e}^{2}+\beta^{2} \sigma_{u}^{2}\left(\sigma_{x}^{2}+\mu_{x}^{2}\right)\right]= \\
& =\frac{\sigma_{u}^{2}}{\mu_{x}^{2}}\left[k_{e}+\beta^{2}\left(\sigma_{u}^{2} k_{x}+\mu_{x}^{2}\right)\right] .
\end{aligned}
$$

Fica assim provada a proposição.

Notamos que a variância assintótica do estimador $\widehat{\beta}_{1}$ não depende das componentes da matriz $\Lambda$; portanto não é afetada pela assimetria.

Proposição 3.15. Considerando um modelo estrutural assimétrico multiplicativo com variância constante, com os parâmetros $\sigma_{u}^{2}$ e $\alpha$ conhecidos, tal que $\alpha=0$. Seja o estimador consistente $\widehat{\beta}_{2}=c_{1}^{-1}\left[\frac{S_{Y X}+\overline{Y X}}{S_{X X}+\bar{X}^{2}}\right]$ (Arellano-Valle e Bolfarine, Gasco, 2001), com $c_{1}=\frac{\sigma_{x}^{2}+\mu_{x}^{2}}{\Delta+\mu_{x}^{2}}$ e $\Delta=\sigma_{X X}^{2}=\sigma_{x}^{2} \sigma_{u}^{2}+\sigma_{x}^{2}+\mu_{x}^{2} \sigma_{u}^{2}$, então a variância assintótica de $\widehat{\beta}_{2}$, é dada por,

$$
\Omega=\frac{1}{\left(\sigma_{x}^{2}+\mu_{x}^{2}\right)^{2}}\left[\mu_{x}^{2} \Omega_{1}+2 \mu_{x} \gamma \Omega_{2}+(3 \delta-1) \Omega_{3}+\delta \Omega_{4}\right],
$$


com,

$$
\begin{aligned}
\Omega_{1} & =\sigma_{Y Y}^{2}+2 \beta\left(1-2 c_{1}\right) \sigma_{Y X}^{2}+\beta^{2}\left(1-2 c_{1}\right)^{2} \sigma_{X X}^{2}= \\
& =\beta^{2} \sigma_{u}^{2} k_{x}\left(3-2 c_{1}\right) \sigma_{u}^{2} k_{e}+\beta^{2} \Delta\left(1-2 c_{1}\right)^{2} \\
\Omega_{2} & =\sum_{j=1}^{m} t_{2 j}\left(t_{1 j}^{2}+\left(1-2 c_{1}\right) t_{1 j} t_{2 j}+\beta^{2} c_{1}\left(1-2 c_{1}\right) t_{2 j}^{2}\right) \zeta_{j} \\
\Omega_{3} & \left.=\left(\sigma_{Y X}^{2}+\beta c_{1} \sigma_{X X}^{2}\right)^{2}=\beta^{2}\left(\sigma_{u}^{2} \lambda_{x}+c_{1}\right) \Delta\right)^{2} \\
\Omega_{4} & =\sum_{j=1}^{m}\left(\kappa_{j}-3\right) t_{2 j}\left(t_{1 j}+\beta c_{1} t_{2 j}\right)^{2}
\end{aligned}
$$

Demonstração. Considerando o Teorema 2.9, temos que,

$$
\sqrt{n}\left(\begin{array}{c}
f(\overline{\mathbf{Z}}-\boldsymbol{\mu}) \\
f\left(\operatorname{vech}\left(\mathbf{S}_{Z}-\mathbf{\Sigma}\right)\right.
\end{array}\right) \stackrel{d}{\rightarrow} N_{5}(\mathbf{0}, \Omega)
$$

onde $\Omega=\Pi \Upsilon \Pi^{\prime}$, com $\Pi=\frac{\partial f}{\partial(\mu, v e c h(\Sigma))^{T}}$ e $\Upsilon=\left(\begin{array}{cc}\Sigma & \Gamma \\ \Gamma^{T} & \Lambda\end{array}\right)$, de modo que,

$$
\Upsilon=\left(\begin{array}{ccccc}
v_{11} & v_{12} & v_{13} & v_{14} & v_{15} \\
& v_{22} & v_{23} & v_{24} & v_{25} \\
& & v_{33} & v_{34} & v_{35} \\
& & & v_{44} & v_{45} \\
& & & & v_{55}
\end{array}\right)=\left(\begin{array}{ccccc}
\sigma_{Y Y} & \sigma_{Y X} & \gamma_{11} & \gamma_{12} & \gamma_{13} \\
& \sigma_{X X} & \gamma_{21} & \gamma_{22} & \gamma_{23} \\
& & \lambda_{11} & \lambda_{12} & \lambda_{13} \\
& & & \lambda_{22} & \lambda_{23} \\
& & & & \lambda_{33}
\end{array}\right) \text {. }
$$

Se $f\left((x, y, z, u, v)^{T}\right)=c_{1}^{-1}\left[\frac{u+x y}{v+y^{2}}\right]$ então, $\Pi=\frac{1}{\sigma_{x}^{2}+\mu_{x}^{2}}\left(\mu_{x}, \beta \mu_{x}\left(1-2 c_{1}\right), 0,1, \beta c_{1}\right)$, e

$$
\sqrt{n}\left(\widehat{\beta}_{2}-\beta\right) \stackrel{d}{\rightarrow} N(0, \Omega) .
$$

Com,

$$
\begin{aligned}
\Omega= & \frac{1}{\left(\sigma_{x}^{2}+\mu_{x}^{2}\right)^{2}}\left[\mu_{x}^{2} v_{11}+2 \beta \mu_{x}^{2}\left(1-2 c_{1}\right) v_{12}+2 \mu_{x} v_{14}+2 \beta \mu_{x} c_{1} v_{15}+\right. \\
& +\beta^{2} \mu_{x}^{2}\left(1-2 c_{1}\right)^{2} v_{22}+2 \beta \mu_{x}\left(1-2 c_{1}\right) v_{24}+2 \beta^{2} \mu_{x}\left(1-2 c_{1}\right) v_{25}+ \\
& \left.+v_{44}+2 \beta c_{1} v_{45}+\beta^{2} c_{1}^{2} v_{55}\right] \\
= & \frac{1}{\left(\sigma_{x}^{2}+\mu_{x}^{2}\right)^{2}}\left[\mu_{x}^{2} \sigma_{Y Y}^{2}+2 \beta \mu_{x}^{2}\left(1-2 c_{1}\right) \sigma_{Y X}^{2}+\beta^{2} \mu_{x}^{2}\left(1-2 c_{1}\right)^{2} \sigma_{X X}^{2}+\right. \\
& +2 \mu_{x} \gamma_{12}+2 \beta \mu_{x} c_{1} \gamma_{13}+2 \beta \mu_{x}\left(1-2 c_{1}\right) \gamma_{22}+2 \beta^{2} \mu_{x}\left(1-2 c_{1}\right) \gamma_{23}+ \\
& \left.+\lambda_{22}+2 \beta c_{1} \lambda_{23}+\beta^{2} c_{1}^{2} \lambda_{33}\right] \\
= & \frac{\mu_{x}^{2}}{\left(\sigma_{x}^{2}+\mu_{x}^{2}\right)^{2}}\left[\sigma_{Y Y}^{2}+2 \beta\left(1-2 c_{1}\right) \sigma_{Y X}^{2}+\beta^{2}\left(1-2 c_{1}\right)^{2} \sigma_{X X}^{2}\right]+ \\
& +\frac{2 \mu_{x} \gamma}{\left(\sigma_{x}^{2}+\mu_{x}^{2}\right)^{2}} 2\left[\sum_{j=1}^{m} t_{2 j}\left(t_{1 j}^{2}+\left(1-2 c_{1}\right) t_{1 j} t_{2 j}+\beta^{2} c_{1}\left(1-2 c_{1}\right) t_{2 j}^{2}\right) \zeta_{j}\right]+ \\
& +\frac{(3 \delta-1)}{\left(\sigma_{x}^{2}+\mu_{x}^{2}\right)^{2}}\left(\sigma_{Y X}^{2}+\beta c_{1} \sigma_{X X}^{2}\right)^{2}+\frac{\delta}{\left(\sigma_{x}^{2}+\mu_{x}^{2}\right)^{2}}\left[\sum_{j=1}^{m}\left(\kappa_{j}-3\right) t_{2 j}\left(t_{1 j}+\beta c_{1} t_{2 j}\right)^{2}\right] \\
= & \frac{\mu_{x}^{2}}{\left(\sigma_{x}^{2}+\mu_{x}^{2}\right)^{2}} \Omega_{1}+\frac{2 \mu_{x} \gamma}{\left(\sigma_{x}^{2}+\mu_{x}^{2}\right)^{2}} 2 \Omega_{2}+\frac{(3 \delta-1)}{\left(\sigma_{x}^{2}+\mu_{x}^{2}\right)^{2}} \Omega_{3}+\frac{\delta}{\left(\sigma_{x}^{2}+\mu_{x}^{2}\right)^{2}} \Omega_{4}= \\
= & \frac{1}{\left(\sigma_{x}^{2}+\mu_{x}^{2}\right)^{2}}\left[\mu_{x}^{2} \Omega_{1}+2 \mu_{x} \gamma \Omega_{2}+(3 \delta-1) \Omega_{3}+\delta \Omega_{4}\right],
\end{aligned}
$$

Ozán, N. S. 
onde,

$$
\begin{aligned}
\Omega_{1} & =\sigma_{Y Y}^{2}+2 \beta\left(1-2 c_{1}\right) \sigma_{Y X}^{2}+\beta^{2}\left(1-2 c_{1}\right)^{2} \sigma_{X X}^{2}= \\
& =\beta^{2} \sigma_{u}^{2} k_{x}\left(3-2 c_{1}\right) \sigma_{u}^{2} k_{e}+\beta^{2} \Delta\left(1-2 c_{1}\right)^{2} \\
\Omega_{2} & =\sum_{j=1}^{m} t_{2 j}\left(t_{1 j}^{2}+\left(1-2 c_{1}\right) t_{1 j} t_{2 j}+\beta^{2} c_{1}\left(1-2 c_{1}\right) t_{2 j}^{2}\right) \zeta_{j} \\
\Omega_{3} & \left.=\left(\sigma_{Y X}^{2}+\beta c_{1} \sigma_{X X}^{2}\right)^{2}=\beta^{2}\left(\sigma_{u}^{2} \lambda_{x}+c_{1}\right) \Delta\right)^{2} \\
\Omega_{4} & =\sum_{j=1}^{m}\left(\kappa_{j}-3\right) t_{2 j}\left(t_{1 j}+\beta c_{1} t_{2 j}\right)^{2} .
\end{aligned}
$$

Portanto, fica assim provada a proposição.

Corolario 3.16. Considerando a distribuição pseudo elíptica temos que,

$$
\Omega=\Omega_{E}=\frac{1}{\left(\sigma_{x}^{2}+\mu_{x}^{2}\right)^{2}}\left[\mu_{x}^{2} \Omega_{1}+2 \mu_{x} \gamma \Omega_{2}+(3 \delta-1) \Omega_{3}\right] .
$$

Considerando a distribuição pseudo normais temos que,

$$
\Omega=\Omega_{N}=\frac{1}{\left(\sigma_{x}^{2}+\mu_{x}^{2}\right)^{2}}\left[\mu_{x}^{2} \Omega_{1}+2 \mu_{x} \gamma \Omega_{2}+2 \Omega_{3}\right],
$$

A seguir, analisamos a variância assintótica do estimador de $\beta$ considerando $\alpha$ desconhecido.

Proposição 3.17. Considerando um modelo estrutural assimétrico multiplicativo variância constante, seja $\widehat{\beta}_{3}$ o estimador consistente para o parâmetro $\beta$, dado por, $\widehat{\beta}_{3}=\frac{S_{Y Y}-\sigma_{e}^{2}}{S_{Y X}}$, com $\sigma_{e}^{2}$ conhecido. Então a variância assintótica de $\widehat{\beta}_{3}, e ́$

$$
\Omega=\frac{(3 \delta-1)}{\beta}\left(\frac{\sigma_{e}^{2}}{\sigma_{x}^{2}}\right)^{2}+\frac{\delta}{\sigma_{x}^{4}} \sum_{j=1}^{m}\left(\kappa_{j}-3\right) t_{1 j}^{2}\left(\frac{1}{\beta} t_{1 j}-t_{2 j}\right)^{2} .
$$

Demonstração. Utilizando o Teorema 2.9, com $f\left((x, y, z)^{\prime}\right)=\frac{x-\sigma_{e}^{2}}{y}$, temos que,

$$
\left.\sqrt{n}\left(\widehat{\beta}_{3}-\beta\right)\right) \stackrel{d}{\rightarrow} N(0, \Omega),
$$

onde $\Omega=\Pi \Lambda \Pi^{\prime}$ e $\Pi=\frac{\partial f}{\partial v e c T^{T}(\Sigma)}$. Assim, $\Pi=\frac{1}{\sigma_{x}^{2}}\left(\frac{1}{\beta},-1,0\right)$, e portanto $\Omega$ é:

$$
\begin{aligned}
\Omega & =\frac{1}{\sigma_{x}^{4}}\left[\frac{1}{\beta^{2}} \lambda_{11}-\frac{2}{\beta} \lambda_{12}+\lambda_{23}\right]= \\
& =\frac{1}{\sigma_{x}^{4}}\left[(3 \delta-1)\left(\frac{1}{\beta} \sigma_{Y Y}^{2}-\sigma_{Y X}^{2}\right)^{2}+\delta \sum_{j=1}^{m}\left(\kappa_{j}-3\right) t_{1 j}^{2}\left(\frac{1}{\beta^{2}} t_{1 j}^{2}-\frac{2}{\beta} t_{1 j} t_{2 j}+t_{2 j}^{2}\right)\right]= \\
& =\frac{(3 \delta-1)}{\beta}\left(\frac{\sigma_{e}^{2}}{\sigma_{x}^{2}}\right)^{2}+\frac{\delta}{\sigma_{x}^{4}} \sum_{j=1}^{m}\left(\kappa_{j}-3\right) t_{1 j}^{2}\left(\frac{1}{\beta} t_{1 j}-t_{2 j}\right)^{2} .
\end{aligned}
$$

Fica assim provada a proposição.

Ozán, N. S. 
Corolario 3.18. No caso de distribuição pseudo elíptica, temos que,

$$
\Omega=\Omega_{E}=\frac{(3 \delta-1)}{\beta^{2}}\left(\frac{\sigma_{x}^{2}}{\sigma_{e}^{2}}\right)^{2}
$$

No caso de distribuição pseudo normal, temos que,

$$
\Omega=\Omega_{N}=\frac{2}{\beta^{2}}\left(\frac{\sigma_{x}^{2}}{\sigma_{e}^{2}}\right)^{2}
$$

Proposição 3.19. Considerando um modelo estrutural assimétrico multiplicativo con variância constante, seja $\widehat{\beta}_{4}$, o estimador consistente para $\beta$, isto é, $\widehat{\beta}_{4}=c_{2}^{-1}\left[\frac{S_{Y X}}{S_{X X}+\bar{X} \sigma_{u}^{2}}\right]$ $\operatorname{com} c_{2}=\frac{\sigma_{x}^{2}}{\Delta+\mu_{x}^{2} \sigma_{u}^{2}}$ e $\Delta=\sigma_{X X}^{2}=\sigma_{x}^{2} \sigma_{u}^{2}+\sigma_{x}^{2}+\mu_{x}^{2} \sigma_{u}^{2}$. Então a variância assintótica de $\widehat{\beta}_{4}, \quad e ́$ dada por,

$$
\Omega=\frac{1}{\left(\sigma_{x}^{2}+\mu_{x}^{2} \sigma_{u}^{2}\right)^{2}}\left[4 \beta \mu_{x} \sigma_{u}^{2}\left(\beta \mu_{x} \sigma_{u}^{2} \Delta-\Omega_{1}\right)+\beta^{2}(3 \delta-1) \Omega_{2}+\delta \Omega_{3}\right]
$$

com,

$$
\begin{aligned}
& \Omega_{1}=\sum_{j=1}^{m} t_{2 j}^{2}\left(c_{2}^{-1} \mu_{x} t_{1 j}+\beta t_{2 j}\right) \zeta_{j}, \\
& \Omega_{2}=\left(c_{2}^{-1} \sigma_{x}^{2}+\Delta\right)^{2}, \\
& \Omega_{3}=\sum_{j=1}^{m}\left(\kappa_{j}-3\right) t_{2 j}^{2}\left(c_{2}^{-1} t_{1 j}+\beta t_{2 j}\right)^{2} .
\end{aligned}
$$

Demonstração. Aplicando o Teorema 2.9, temos que,

$$
\sqrt{n}\left(\begin{array}{c}
f(\overline{\mathbf{Z}}-\boldsymbol{\mu}) \\
f\left(\operatorname{vech}\left(\mathbf{S}_{Z}-\mathbf{\Sigma}\right)\right.
\end{array}\right) \stackrel{d}{\rightarrow} N_{5}(\mathbf{0}, \Omega)
$$

onde $\Omega=\Pi \Upsilon \Pi^{T}$, com $\Pi=\frac{\partial f}{\partial(\mu, v e c h(\Sigma))^{T}}$ e $\Upsilon=\left(\begin{array}{cc}\Sigma & \Gamma \\ \Gamma^{T} & \Lambda\end{array}\right)$ como em (3.51). Se $f\left((x, y, z, u, v)^{T}\right)=c_{2}^{-1}\left[\frac{u}{v+y^{2} \sigma_{u}^{2}}\right]$ então, $\Pi=\frac{1}{\sigma_{x}^{2}+\mu_{x}^{2} \sigma_{u}^{2}}\left(0,-2 \beta \mu_{x} \sigma_{u}^{2}, 0, c_{2}^{-1}, \beta\right)$, e

$$
\sqrt{n}\left(\widehat{\beta}_{2}-\beta\right) \stackrel{d}{\rightarrow} N(0, \Omega)
$$

Ozán, N. S. 
Portanto,

$$
\begin{aligned}
\Omega= & \frac{1}{\left(\sigma_{x}^{2}+\mu_{x}^{2} \sigma_{u}^{2}\right)^{2}}\left[4 \beta^{2} \mu_{x}^{2} \sigma_{u}^{4} v_{22}-4 \beta^{2} \mu_{x}^{2} \sigma_{u}^{2} c_{2}^{-1} v_{24}-4 \beta^{2} \mu_{x} \sigma_{u}^{2} v_{25}+\right. \\
& \left.+c_{2}^{-2} v_{44}+2 \beta c_{2}^{-1} v_{45}+\beta^{2} v_{55}\right]= \\
= & \frac{1}{\left(\sigma_{x}^{2}+\mu_{x}^{2} \sigma_{u}^{2}\right)^{2}}\left[4 \beta^{2} \mu_{x}^{2} \sigma_{u}^{4} \sigma_{X X}^{2}-4 \beta \mu_{x} \sigma_{u}^{2}\left(c_{2}^{-1} \mu_{x} \gamma_{22}+\beta \gamma_{25}\right)+\right. \\
& \left.+c_{2}^{-2} \lambda_{22}+2 \beta c_{2}^{-1} \lambda_{23}+\beta^{2} \lambda_{33}\right]={ }_{m}^{m} \\
= & \frac{1}{\left(\sigma_{x}^{2}+\mu_{x}^{2} \sigma_{u}^{2}\right)^{2}}\left[4 \beta^{2} \mu_{x}^{2} \sigma_{u}^{4} \Delta-4 \beta \mu_{x} \sigma_{u}^{2} \sum_{j=1}^{2} t_{2 j}^{2}\left(c_{2}^{-1} \mu_{x} t_{1 j}+\beta t_{2 j}\right) \zeta_{j}\right]+ \\
& +\frac{\beta^{2}(3 \delta-1)}{\left(\sigma_{x}^{2}+\mu_{x}^{2} \sigma_{u}^{2}\right)^{2}}\left(c_{2}^{-1} \sigma_{x}^{2}+\Delta\right)^{2}+ \\
& +\frac{\delta}{\left(\sigma_{x}^{2}+\mu_{x}^{2} \sigma_{u}^{2}\right)^{2}} \sum_{j=1}^{m}\left(\kappa_{j}-3\right) t_{2 j}^{2}\left(c_{2}^{-2} t_{1 j}^{2}+2 \beta c_{2}^{-1} t_{1 j} t_{2 j}+\beta^{2} t_{2 j}^{2}\right)= \\
= & \frac{4 \beta \mu_{x} \sigma_{u}^{2}}{\left(\sigma_{x}^{2}+\mu_{x}^{2} \sigma_{u}^{2}\right)^{2}}\left[\beta \mu_{x} \sigma_{u}^{2} \Delta-\sum_{j=1}^{m} t_{2 j}^{2}\left(c_{2}^{-1} \mu_{x} t_{1 j}+\beta t_{2 j}\right) \zeta_{j}\right]+ \\
& +\frac{\beta^{2}(3 \delta-1)}{\left(\sigma_{x}^{2}+\mu_{x}^{2} \sigma_{u}^{2}\right)^{2}}\left(c_{2}^{-1} \sigma_{x}^{2}+\Delta\right)^{2}+\frac{\delta}{\left(\sigma_{x}^{2}+\mu_{x}^{2} \sigma_{u}^{2}\right)^{2}} \sum_{j=1}^{m}\left(\kappa_{j}-3\right) t_{2 j}^{2}\left(c_{2}^{-1} t_{1 j}+\beta t_{2 j}\right)^{2}= \\
= & \frac{4 \beta \mu_{x} \sigma_{u}^{2}}{\left(\sigma_{x}^{2}+\mu_{x}^{2} \sigma_{u}^{2}\right)^{2}}\left[\beta \mu_{x} \sigma_{u}^{2} \Delta-\Omega_{1}+\frac{\beta^{2}(3 \delta-1)}{\left(\sigma_{x}^{2}+\mu_{x}^{2} \sigma_{u}^{2}\right)^{2}} \Omega_{2}+\frac{\delta}{\left(\sigma_{x}^{2}+\mu_{x}^{2} \sigma_{u}^{2}\right)^{2}} \Omega_{3},\right.
\end{aligned}
$$

onde,

$$
\begin{aligned}
& \Omega_{1}=\sum_{j=1}^{m} t_{2 j}^{2}\left(c_{2}^{-1} \mu_{x} t_{1 j}+\beta t_{2 j}\right) \zeta_{j} \\
& \Omega_{2}=\left(c_{2}^{-1} \sigma_{x}^{2}+\Delta\right)^{2} \\
& \Omega_{3}=\sum_{j=1}^{m}\left(\kappa_{j}-3\right) t_{2 j}^{2}\left(c_{2}^{-1} t_{1 j}+\beta t_{2 j}\right)^{2}
\end{aligned}
$$

Deste modo fica provada a proposição.

Corolario 3.20. Se assumimos distribuição pseudo elípticas, temos que,

$$
\Omega=\Omega_{E}=\frac{1}{\left(\sigma_{x}^{2}+\mu_{x}^{2} \sigma_{u}^{2}\right)^{2}}\left[4 \beta \mu_{x} \sigma_{u}^{2}\left(\beta \mu_{x} \sigma_{u}^{2} \Delta-\Omega_{1}\right)+\beta^{2}(3 \delta-1) \Omega_{2}\right] .
$$

Se assumimos distribuição pseudo normal, temos que,

$$
\Omega=\Omega_{N}=\frac{1}{\left(\sigma_{x}^{2}+\mu_{x}^{2} \sigma_{u}^{2}\right)^{2}}\left[4 \beta \mu_{x} \sigma_{u}^{2}\left(\beta \mu_{x} \sigma_{u}^{2} \Delta-\Omega_{1}\right)+2 \beta^{2} \Omega_{2}\right]
$$

A seguir, apresentamos um resumo dos resultados obtidos para as variância assintótica. dos estimadores propostos para o parâmetro $\beta$.

Ozán, N. S. 
Tabela 3.1 Estimadores Modelo Assimétrico Aditivo

\begin{tabular}{cc}
\hline \hline Estimador & Variância Assintótica \\
\hline \hline$\sigma_{u}^{2}$ conhecida & $\Omega=\beta^{2}(3 \delta-1) \frac{\sigma_{u}^{4}}{\sigma_{x}^{4}}+\frac{\delta}{\sigma_{x}^{4}} \Omega_{1}$ \\
$\widehat{\beta}_{1}=\frac{S_{X Y}}{S_{X X}-\sigma_{u}^{2}}$. & $\Omega_{1}=\sum_{j=1}^{m}\left(\kappa_{j}-3\right) t_{2 j}^{2}\left(t_{1 j}-\beta t_{2 j}\right)^{2}$ \\
& \\
$\widehat{\beta}_{2}=\frac{S_{Y Y}-k_{e} S_{X X}-\left[\left(S_{Y Y}-k_{e} S_{X X}\right)^{2}+4 S_{Y X}^{2}\right]^{\frac{1}{2}}}{2 S_{Y X}}$ & $\Omega=(3 \delta-1)\left(a \sigma_{Y Y}+b \sigma_{Y X}+c \sigma_{X X}\right)^{2}+$ \\
& $+\delta \sum_{j=1}^{m}\left(\kappa_{j}-3\right)\left(a t_{1 j}^{2}+b t_{1 j} t_{2 j}+c t_{2 j}^{2}\right)^{2}$ \\
& $a=\frac{1+\left(\sigma_{Y Y}^{2}-k_{e} \sigma_{X X}^{2}\right)\left[\left(\sigma_{Y Y}^{2}-k_{e} \sigma_{X X}^{2}\right)^{2}+4 \sigma_{Y X}^{4}\right]^{-\frac{1}{2}}}{2 \sigma_{Y X}^{2}}$ \\
& $b=\frac{-\sigma_{Y Y}^{2}+k_{e} \sigma_{X X}^{2}-\left[\left(\sigma_{Y Y}^{2}-k_{e} \sigma_{X X}^{2}\right)^{2}+4 \sigma_{Y X}^{4}\right]^{-\frac{1}{2}}}{2 \sigma_{Y X}^{4}}+$ \\
& $c=\frac{-k_{e}+\left(\sigma_{Y Y}^{2}-k_{e} \sigma_{X X}^{2}\right) k_{e}\left[\left(\sigma_{Y Y}^{2}-k_{e} \sigma_{X X}^{2}\right)^{2}+4 \sigma_{Y X}^{4}\right]^{-\frac{1}{2}}}{2 \sigma_{Y X}^{2}}$ \\
\hline \hline
\end{tabular}

Tabela 3.2 Estimadores Modelo Assimétrico Multiplicativo, $\alpha=0$

\begin{tabular}{cc}
\hline \hline Estimador & Variância Assintótica \\
\hline \hline$\widehat{\beta}_{1}=\frac{\bar{Y}}{\bar{X}}$ & $\Omega=\frac{\sigma_{u}^{2}}{\mu_{x}^{2}}\left[k_{e}+\beta^{2}\left(\sigma_{u}^{2} k_{x}+\mu_{x}^{2}\right)\right]$. \\
$\widehat{\beta}_{2}=c_{1}^{-1}\left[\frac{S_{Y X}+\overline{Y X}}{S_{X X X}+\bar{X}^{2}}\right]$ & $\Omega=\frac{1}{\left(\sigma_{x}^{2}+\mu_{x}^{2}\right)^{2}}\left[\mu_{x}^{2} \Omega_{1}+2 \mu_{x} \gamma \Omega_{2}+(3 \delta-1) \Omega_{3}+\delta \Omega_{4}\right]$ \\
$c_{1}=\frac{\sigma_{x}^{2}+\mu_{x}^{2}}{\Delta+\mu_{x}^{2}}$ & $\Omega_{1}=\sigma_{Y Y}^{2}+2 \beta\left(1-2 c_{1}\right) \sigma_{Y X}^{2}+\beta^{2}\left(1-2 c_{1}\right)^{2} \sigma_{X X}^{2}$ \\
& $\Omega_{2}=\sum_{j=1}^{m} t_{2 j}\left(t_{1 j}^{2}+\left(1-2 c_{1}\right) t_{1 j} t_{2 j}+\beta^{2} c_{1}\left(1-2 c_{1}\right) t_{2 j}^{2}\right) \zeta_{j}$ \\
$\left.\Omega_{3}=\left(\sigma_{Y X}^{2}+\beta c_{1} \sigma_{X X}^{2}\right)^{2}=\beta^{2}\left(\sigma_{u}^{2} \lambda_{x}+c_{1}\right) \Delta\right)^{2}$ \\
$\Omega_{4}=\sum_{j=1}^{{ }_{m}}\left(\kappa_{j}-3\right) t_{2 j}\left(t_{1 j}+\beta c_{1} t_{2 j}\right)^{2}$ \\
$\Delta=\sigma_{X X}^{2}=\sigma_{x}^{2} \sigma_{u}^{2}+\sigma_{x}^{2}+\mu_{x}^{2} \sigma_{u}^{2}$ \\
\hline \hline
\end{tabular}

Ozán, N. S. 
Tabela 3.3 Estimadores Modelo Assimétrico Multiplicativo, $\alpha$ conhecido

\begin{tabular}{|c|c|}
\hline Estimador & Variância Assintótica \\
\hline$\widehat{\beta}_{3}=\frac{S_{Y Y}-\sigma_{e}^{2}}{S_{Y X}}$ & $\Omega=\frac{(3 \delta-1)}{\beta^{2}}\left(\frac{\sigma_{x}^{2}}{\sigma_{e}^{2}}\right)^{2}+\frac{\delta}{\sigma_{e}^{2}}\left(\frac{\sigma_{x}^{2}}{\sigma_{e}^{2}}\right)^{2} \sum_{j=1}^{m}\left(\kappa_{j}-3\right) t_{1 j}^{2}\left(\frac{1}{\beta} t_{1 j}-t_{2 j}\right)^{2}$ \\
\hline $\begin{array}{c}\widehat{\beta}_{4}=c_{2}^{-1}\left[\frac{S_{Y X}}{S_{X X}+\bar{X} \sigma_{u}^{2}}\right] \\
c_{2}=\frac{\sigma_{x}^{2}}{\Delta+\mu_{x}^{2} \sigma_{u}^{2}}\end{array}$ & $\begin{array}{c}\Omega=\frac{1}{\left(\sigma_{x}^{2}+\mu_{x}^{2} \sigma_{u}^{2}\right)^{2}}\left[4 \beta \mu_{x} \sigma_{u}^{2}\left(\beta \mu_{x} \sigma_{u}^{2} \Delta-\Omega_{1}\right)+\beta^{2}(3 \delta-1) \Omega_{2}+\delta \Omega_{3}\right] \\
\Omega_{1}=\sum_{j=1}^{m} t_{2 j}^{2}\left(c_{2}^{-1} \mu_{x} t_{1 j}+\beta t_{2 j}\right) \zeta_{j} \\
\Omega_{2}=\left(c_{2}^{-1} \sigma_{x}^{2}+\Delta\right)^{2} \\
\Omega_{3}=\sum_{j=1}^{m}\left(\kappa_{j}-3\right) t_{2 j}^{2}\left(c_{2}^{-1} t_{1 j}+\beta t_{2 j}\right)^{2} \\
\Delta \stackrel{\sigma_{X X}^{2}=\sigma_{x}^{2}}{=} \sigma_{u}^{2} \sigma_{x}^{2}+\sigma_{x}^{2}+\mu_{x}^{2} \sigma_{u}^{2} .\end{array}$ \\
\hline
\end{tabular}




\section{Modelo Normal Assimétrico com Erros nas Variáveis}

\subsection{Introdução}

Neste capítulo apresentamos o modelo normal assimétrico com erros nas variáveis, em particular o caso do modelo estrutural linear aditivo.

$\mathrm{Na}$ Seção 2, derivamos a função de verossimilhança correspondente a amostra obsèrvada $\mathbf{Z}_{1}=\left(Y_{1}, X_{1}\right)^{T}, \ldots, \mathbf{Z}_{n}=\left(Y_{n}, X_{n}\right)^{T}$, através da integração das variáveis não observadas $x_{1}, \ldots, x_{n}$. É interessante ressaltar que pode-se obter uma forma analítica fechada para esta verossimilhança, o que permite a implementação de técnicas computacionais para a obtenção dos estimadores de máxima verossimilhança e também para obtenção da matriz Hessiana, o que leva a estimativas da matriz de covariâncias amostrais destes estimadores.

Na Seção 3 obtemos a distribuição assintótica conjunta do vetor de médias amostrais $\overline{\mathbf{Z}}=\frac{1}{n} \sum_{i=1}^{n} \mathbf{Z}_{i}$ e a matriz de covariâncias amostrais $\mathbf{S}_{Z}=\frac{1}{n} \sum_{i=1}^{n}\left(\mathbf{Z}_{i}-\overline{\mathbf{Z}}\right)\left(\mathbf{Z}_{i}-\overline{\mathbf{Z}}\right)^{T}$. Este resultado é importante porque através dele estudamos o comportamento assintótico de estimadores de momentos obtidos sob as suposições de normalidade usual.

\subsection{Modelo Estrutural Normal Assimétrico}

\subsubsection{Especificação do Modelo}

A notação usada para um modelo estrutural linear aditivo com erros nas variáveis (Fuller (1987), Arellano-Valle, Bolfarine, Vilca-Labra (1996), entre outros), para uma amostra de $n$ observações, é:

$$
\left\{\begin{array}{l}
Y_{i}=\alpha+\beta x_{i}+e_{i} \\
X_{i}=x_{i}+u_{i}
\end{array}\right.
$$

onde $\left(Y_{i}, X_{i}\right)^{T}$ são as variáveis observadas, com $e_{i} \sim\left(0, \sigma_{e_{i}}^{2}\right), u_{i} \sim\left(0, \sigma_{u_{i}}^{2}\right)$ sendo os erros aleatórios do modelo, independentes, e $x_{i} \sim\left(\mu_{x_{i}}, \sigma_{x_{i}}^{2}\right)$, covariável não observada, independentes, para $i=1, \ldots, n$. 
Sob a hipótese de modelo estrutural temos que $\mu_{x_{i}}=\mu_{x}, i=1, \ldots, n$. Além disso considerase a variância constante, isto é, $\sigma_{x_{i}}^{2}=\sigma_{x}^{2}, \sigma_{e_{i}}^{2}=\sigma_{e}^{2}$ e $\sigma_{u_{i}}^{2}=\sigma_{u}^{2}$ para $i=1, \ldots, n$.

Portanto, no modelo estrutural assumimos que,

$$
x_{i} \stackrel{i i d}{\sim}\left(\mu_{x}, \sigma_{x}^{2}\right), \quad e_{i} \stackrel{i i d}{\sim}\left(0, \sigma_{e}^{2}\right), \quad u_{i} \stackrel{i i d}{\sim}\left(0, \sigma_{u}^{2}\right),
$$

com $x_{i}, e_{i}$ e $u_{i}$ independentes para todo $i=1, \ldots, n$.

Notando que o modelo (4.1) pode ser escrito como,

$$
\left\{\begin{array}{l}
Y_{i}=\alpha+\beta\left(x_{i}-\mu_{x}\right)+\beta \mu_{x}+e_{i}, \\
X_{i}=x_{i}-\mu_{x}+\mu_{x}+u_{i}
\end{array}\right.
$$

$i=1, \ldots, n$, e definindo $v_{i}=x_{i}-\mu_{x}$, temos que,

$$
\left\{\begin{array}{l}
Y_{i}=\alpha+\beta \mu_{x}+\beta v_{i}+e_{i} \\
X_{i}=\mu_{x}+v_{i}+u_{i}
\end{array}\right.
$$

Em forma matricial o modelo (4.3) fica,

$$
\left(\begin{array}{c}
Y_{i} \\
X_{i}
\end{array}\right)=\left(\begin{array}{c}
\alpha+\beta \mu_{x} \\
\mu_{x}
\end{array}\right)+\left(\begin{array}{ccc}
\beta & 1 & 0 \\
1 & 0 & 1
\end{array}\right)\left(\begin{array}{c}
v_{i} \\
e_{i} \\
u_{i}
\end{array}\right)
$$

ou

$$
\mathrm{Z}_{i}=\mu+\mathbf{B r}_{i}
$$

$i=1, \ldots, n$, onde,

$$
\mathbf{Z}_{i}=\left(\begin{array}{c}
Y_{i} \\
X_{i}
\end{array}\right), \quad \boldsymbol{\mu}=\left(\begin{array}{c}
\alpha+\beta \mu_{x} \\
\mu_{x}
\end{array}\right), \quad \mathbf{r}_{i}=\left(\begin{array}{c}
v_{i} \\
e_{i} \\
u_{i}
\end{array}\right)=\left(\begin{array}{c}
x_{i}-\mu_{x} \\
e_{i} \\
u_{i}
\end{array}\right), \quad \mathbf{B}=\left(\begin{array}{ccc}
\beta & 1 & 0 \\
1 & 0 & 1
\end{array}\right) .
$$

A suposição feita para a distribuição dos componentes do vetor $\mathbf{r}_{i}$ no modelo normal assimétrico é de assumir uma distribuição normal assimétrica segundo a Definição 2.12 no Capítulo 2. Isto é,

$$
v_{i} \stackrel{i i d}{\sim} S N\left(0, \sigma_{x}^{2}, \lambda_{x}\right), \quad e_{i} \stackrel{i i d}{\sim} S N\left(0, \sigma_{e}^{2}, \lambda_{e}\right), \quad u_{i} \stackrel{i i d}{\sim} S N\left(0, \sigma_{u}^{2}, \lambda_{u}\right)
$$

com $v_{i}, e_{i}$ e $u_{i}$ independentes para todo $i=1, \ldots, n$.

Portanto, $\mathbf{r}_{i}=\left(v_{i}, e_{i}, u_{i}\right)^{T}$ tem distribuição normal assimétrica independente com parâmetro de locação 0 , de escala $\Psi$ e de assimetria $\lambda$.

Logo, $\mathbf{r}_{i} \stackrel{\text { iid }}{\sim} S N I_{3}(0, \Psi, \boldsymbol{\lambda}), i=1, \ldots, n, \operatorname{com} \Psi=\operatorname{diag}\left\{\sigma_{x}^{2}, \sigma_{e}^{2}, \sigma_{u}^{2}\right\}$ e $\boldsymbol{\lambda}=\left(\lambda_{x}, \lambda_{e}, \lambda_{u}\right)^{T}$. De modo que aplicando a Definição 2.13, a função de densidade é dada por

$$
f_{\mathrm{r}}\left(\mathbf{r}_{i}\right)=f_{(v, e, u)}\left(v_{i}, e_{i}, u_{i}\right)=\frac{2^{3}}{\sigma_{x}^{2} \sigma_{e}^{2} \sigma_{u}^{2}} \phi_{3}\left(\frac{v_{i}}{\sigma_{x}}, \frac{e_{i}}{\sigma_{e}}, \frac{u_{i}}{\sigma_{u}}\right) \Phi_{3}\left(\frac{\lambda_{x} v_{i}}{\sigma_{x}}, \frac{\lambda_{e} e_{i}}{\sigma_{e}}, \frac{\lambda_{u} u_{i}}{\sigma_{u}}\right) .
$$

Na Seção 4.2.3. temos uma extensão deste modelo, onde consideramos assimetrias diferentes para cada observação. 


\subsubsection{A Função de Verossimilhança}

Sendo $\mathrm{Z}_{1}, \ldots, \mathrm{Z}_{n}$, independentes, segundo o modelo (4.4) e assumindo uma distribuição normal assimétrica independente tri-variada para os vetores $\mathbf{r}_{i}$ determina-se a função de verossimilhança para as observações $\mathbf{Z}_{i}, i=1, \ldots, n$, do modelo com erros nas variáveis.

Observação 7. Para representar a função de densidade e a função de distribuição acumulada, respectivamente, de uma distribuição normal de dimensão $p$ de parâmetros $(\boldsymbol{\mu}, \boldsymbol{\Sigma})$, usamos a seguinte notação,

- $\phi_{p}(\mathbf{z} \mid \boldsymbol{\mu}, \Sigma)=|\Sigma|^{-\frac{1}{2}} \phi_{p}\left(\Sigma^{-\frac{1}{2}}(\mathbf{z}-\mu)\right), \mathrm{e}$

- $\Phi_{p}(\mathbf{z} \mid \boldsymbol{\mu}, \boldsymbol{\Sigma})=\Phi_{p}\left(\boldsymbol{\Sigma}^{-\frac{1}{2}}(\mathbf{z}-\boldsymbol{\mu})\right)$, quando $\boldsymbol{\Sigma}$ é diagonal.

Temos então como conseqüência as seguintes identidades:

Propriedade 2. Seja $\mathrm{z} \sim N_{p}(\mu, \Sigma)$, então,

- $\phi_{p}(\mathbf{z}+a \mid \mu, \Sigma)=\phi_{p}(\mathbf{z} \mid \mu-a, \Sigma)$.

- $\Phi_{p}(\mathbf{z}+a \mid \boldsymbol{\mu}, \boldsymbol{\Sigma})=\Phi_{p}(\mathbf{z} \mid \boldsymbol{\mu}-a, \mathbf{\Sigma})$.

Derivamos a seguir a função de densidade para uma única observação, onde omite-se o índice $i$ para simplificar a notação.

Teorema 4.1. Seja $\mathbf{Z}=\boldsymbol{\mu}+\mathrm{Br}$, como em (4.4), onde $\mathbf{r}=(v, e, u)^{T} \stackrel{\text { ind }}{\sim} \operatorname{SNI}_{3}(0, \Psi, \lambda)$, $\operatorname{com} \Psi=\operatorname{diag}\left\{\sigma_{x}^{2}, \sigma_{e}^{2}, \sigma_{u}^{2}\right\}$ e $\lambda=\left(\lambda_{x}, \lambda_{e}, \lambda_{u}\right)^{T}$, então a função de densidade para $\mathrm{Z} e ́$,

$$
f_{Z}(\mathbf{z})=2^{3}|\Sigma|^{-\frac{1}{2}} \phi_{2}\left(\Sigma^{-\frac{1}{2}}(\mathbf{z}-\boldsymbol{\mu})\right) \Phi_{3}\left(\Delta_{*} \Sigma^{-\frac{1}{2}}(\mathbf{z}-\boldsymbol{\mu}) \mid 0,\left(\Psi+\frac{\Delta \mathbf{b b}^{T} \Delta}{\mathbf{b}^{T} \Psi^{-1} \mathbf{b}}\right)\right)
$$

com,

- $\boldsymbol{\mu}=\left(\begin{array}{c}\alpha+\beta \mu_{x} \\ \mu_{x}\end{array}\right)$ e $\boldsymbol{\Sigma}=\left(\begin{array}{cc}\beta^{2} \Sigma_{x}^{2}+\sigma_{e}^{2} & \beta \sigma_{x}^{2} \\ \beta \sigma_{x}^{2} & \sigma_{x}^{2}+\sigma_{u}^{2}\end{array}\right)$

- $\phi_{2}\left(\boldsymbol{\Sigma}^{-\frac{1}{2}}(\mathbf{z}-\boldsymbol{\mu})\right)$ a função de densidade de uma distribuição $N_{2}\left(0, \mathbf{I}_{2}\right)$ avaliada em $\Sigma^{-\frac{1}{2}}(\mathrm{z}-\mu)$

- $\Phi_{3}\left(\Delta_{*} \boldsymbol{\Sigma}^{-\frac{1}{2}}(\mathbf{z}-\boldsymbol{\mu})\right)$, função de distribuição acumulada de uma distribuição normal de dimensão 3 , avaliada em $\Delta_{*} \boldsymbol{\Sigma}^{-\frac{1}{2}}(\mathbf{z}-\boldsymbol{\mu})$,

- $\Delta_{*}=\Delta \Psi B^{T} \Sigma^{-\frac{1}{2}}, \Delta=\operatorname{Diag}\left\{\lambda_{x}, \lambda_{e}, \lambda_{u}\right\}, \mathbf{b}=(-1, \beta, 1)^{T}$. 
Demonstração. Temos que,

$$
x \sim S N\left(\mu_{x}, \sigma_{x}^{2}, \lambda_{x}\right), \quad e \sim S N\left(0, \sigma_{e}^{2}, \lambda_{e}\right), \quad u \sim S N\left(0, \sigma_{u}^{2}, \lambda_{u}\right),
$$

isto implica que a distribuição das variáveis observadas condicionada à covariável não observada são normais assimétricas, ou seja,

$$
Y\left|x \sim S N\left(\alpha+\beta x, \sigma_{e}^{2}, \lambda_{e}\right), \quad X\right| x \sim S N\left(x, \sigma_{u}^{2}, \lambda_{u}\right) .
$$

Portanto, a função de densidade conjunta (condicional) de $(Y, X) \mid x$ é,

$$
f(Y, X \mid x)=\frac{2^{2}}{\sigma_{e} \sigma_{u}} \phi\left(\frac{Y-\alpha-\beta x}{\sigma_{e}}\right) \phi\left(\frac{X-x}{\sigma_{u}}\right) \Phi\left(\lambda_{e} \frac{Y-\alpha-\beta x}{\sigma_{e}}\right) \Phi\left(\lambda_{u} \frac{X-x}{\sigma_{u}}\right) .
$$

Assim a função de densidade conjunta é dada por,

$$
\begin{aligned}
f_{(Y, X)}(Y, X) & =\int_{-\infty}^{\infty} f(Y, X \mid x) f(x) d x= \\
& =\int_{-\infty}^{\infty} \begin{array}{l}
\frac{2^{3}}{\sigma_{e} \sigma_{u} \sigma_{x}} \phi\left(\frac{Y-\alpha-\beta x}{\sigma_{e}}\right) \phi\left(\frac{X-x}{\sigma_{u}}\right) \phi\left(\frac{x-\mu_{x}}{\sigma_{x}}\right) \\
\Phi\left(\lambda_{e} \frac{Y-\alpha-\beta x}{\sigma_{e}}\right) \Phi\left(\lambda_{u} \frac{X-x}{\sigma_{u}}\right) \Phi\left(\lambda_{x} \frac{x-\mu_{x}}{\sigma_{x}}\right) d x
\end{array}
\end{aligned}
$$

que pode ser escrita como,

$$
\begin{aligned}
f_{(Y, X)}(Y, X)=\frac{2^{3}}{\sigma_{e} \sigma_{u} \sigma_{x}} \int_{-\infty}^{\infty} & \phi\left(\frac{Y-\alpha-\beta \mu_{x}-\beta\left(x-\mu_{x}\right)}{\sigma_{e}}\right) \Phi\left(\lambda_{e} \frac{Y-\alpha-\beta \mu_{x}-\beta\left(x-\mu_{x}\right)}{\sigma_{e}}\right) \\
& \phi\left(\frac{X-\mu_{x}-\left(x-\mu_{x}\right)}{\sigma_{u}}\right) \phi\left(\frac{x-\mu_{x}}{\sigma_{x}}\right) \Phi\left(\lambda_{u} \frac{X-\mu_{x}-\left(x-\mu_{x}\right)}{\sigma_{u}}\right) \Phi\left(\lambda_{x} \frac{x-\mu_{x}}{\sigma_{x}}\right) d x .
\end{aligned}
$$

De acordo com a notação matricial do modelo em (4.4), temos que

$$
\mathbf{Z}=\left(\begin{array}{c}
Y \\
X
\end{array}\right) \quad \text { e } \quad \boldsymbol{\mu}=\left(\begin{array}{c}
\alpha+\beta \mu_{x} \\
\mu_{x}
\end{array}\right) .
$$

Além disso, considerando a transformação $\bar{e}=Y-\left(\alpha+\beta \mu_{x}\right), \bar{u}=X-\mu_{x}$ e $v=x-\mu_{x}$, onde $d v=d x$, temos que $\mathbf{Z}-\boldsymbol{\mu}=\left(\begin{array}{c}\bar{e} \\ \bar{u}\end{array}\right)$, de modo que,

$$
\left.f_{Z}(\mathbf{z})=\frac{2^{3}}{\sigma_{e} \sigma_{u} \sigma_{x}} \int_{-\infty}^{\infty} \phi\left(\frac{v}{\sigma_{x}}\right) \phi\left(\frac{\bar{e}-\beta v}{\sigma_{e}}\right) \phi\left(\frac{\bar{u}-v}{\sigma_{u}}\right) \Phi\left(\lambda_{x} \frac{v}{\sigma_{x}}\right) \Phi\left(\lambda_{e} \frac{\bar{e}-\beta v}{\sigma_{e}}\right)\right) \Phi\left(\lambda_{u} \frac{\bar{u}-v)}{\sigma_{u}}\right) d v
$$

Definindo $\mathbf{w}=(0, \bar{e}, \bar{u})^{T}, \mathbf{b}=(-1, \beta, 1)^{T}$ e sendo $\Psi=\operatorname{Diag}\left\{\sigma_{x}^{2}, \sigma_{e}^{2}, \sigma_{u}^{2}\right\}$, $\Delta=\operatorname{Diag}\left\{\lambda_{x}, \lambda_{e}, \lambda_{u}\right\}$, escrevemos,

$$
\begin{array}{ll}
\left(\frac{v}{\sigma_{x}}, \frac{\bar{e}-\beta v}{\sigma_{e}}, \frac{\bar{u}-v}{\sigma_{u}}\right)^{T} & =\Psi^{-\frac{1}{2}}(\mathrm{w}-\mathrm{b} v) \\
\left(\lambda_{x} \frac{v}{\sigma_{x}}, \lambda_{e} \frac{\bar{e}-\beta v}{\sigma_{e}}, \lambda_{u} \frac{\bar{u}-v}{\sigma_{u}}\right)^{T} & =\Psi^{-\frac{1}{2}} \Delta(\mathrm{w}-\mathrm{b} v) .
\end{array}
$$

Assim, analisando o integrando em (4.8) temos, respectivamente, para a função de densidade e a função de distribuição acumulada, as seguintes identidades: 
1.

$$
\begin{aligned}
\phi\left(\frac{v}{\sigma_{x}}\right) \phi\left(\frac{\bar{e}-\beta v}{\sigma_{e}}\right) \phi\left(\frac{\bar{u}-v}{\sigma_{u}}\right) & =\phi_{3}\left(\left(\frac{v}{\sigma_{x}}, \frac{\bar{e}-\beta v}{\sigma_{e}}, \frac{\bar{u}-v}{\sigma_{u}}\right)^{T}\right) \\
& =\phi_{3}\left(\Psi^{-\frac{1}{2}}(\mathbf{w}-\mathbf{b} v)\right) \\
\text { (propriedades) } & =|\Psi|^{-\frac{1}{2}} \phi_{3}(\mathbf{w}-\mathbf{b} v \mid 0, \Psi) .
\end{aligned}
$$

2.

$$
\begin{aligned}
\Phi\left(\lambda_{x} \frac{v}{\sigma_{x}}\right) \Phi\left(\lambda_{e} \frac{\bar{e}-\beta v}{\sigma_{e}}\right) \Phi\left(\lambda_{u} \frac{\bar{u}-v)}{\sigma_{u}}\right) & =\Phi_{3}\left(\left(\lambda_{x} \frac{v}{\sigma_{x}}, \lambda_{e} \frac{\bar{e}-\beta v}{\sigma_{e}}, \lambda_{u} \frac{\bar{u}-v}{\sigma_{u}}\right)^{T}\right) \\
& =\Phi_{3}\left(\Psi^{-\frac{1}{2}} \Delta(\mathbf{w}-\mathbf{b} v)\right) \\
\text { (propriedades) } & =\Phi_{3}(\Delta \mathbf{w} \mid \Delta \mathbf{b} v, \Psi) \\
& =\int_{\mathbf{t}<\Delta \mathbf{w}} \phi_{3}(\mathbf{t}-\Delta \mathbf{b} v \mid 0, \Psi) d \mathbf{t} .
\end{aligned}
$$

Substituindo as expressões acima em (4.8) temos,

$$
\begin{aligned}
f_{\mathbf{Z}}(\mathbf{z}) & =\frac{2^{3}}{|\Psi|^{-\frac{1}{2}}} \int_{-\infty}^{\infty}|\Psi|^{-\frac{1}{2}} \phi_{3}(\mathbf{w}-\mathbf{b} v \mid 0, \Psi)\left[\int_{\mathbf{t}<\Delta \mathbf{w}} \phi_{3}(\mathbf{t}-\Delta \mathbf{b} v \mid 0, \Psi) d \mathbf{t}\right] d v \\
& =2^{3} \int_{\mathbf{t}<\Delta \mathbf{w}} \int_{-\infty}^{\infty} \phi_{3}(\mathbf{w}-\mathbf{b} v \mid 0, \Psi) \phi_{3}(\mathbf{t}-\Delta \mathbf{b} v \mid 0, \Psi) d v d \mathbf{t} \\
& =2^{3} \int_{\mathbf{t}<\Delta \mathbf{w}} \int_{-\infty}^{\infty} \frac{(2 \pi)^{-\frac{6}{2}}}{|\Psi|} e^{-\frac{1}{2}\left[(\mathbf{w}-\mathbf{b} v)^{T} \Psi^{-1}(\mathbf{w}-\mathbf{b} v)+(\mathbf{t}-\Delta \mathbf{b} v)^{T} \Psi^{-1}(\mathbf{t}-\Delta \mathbf{b} v)\right]} d v d \mathbf{t}
\end{aligned}
$$

A seguir utilizamos a seguinte notação,

$$
Q(v \mid \mathbf{w}, \mathbf{t})=(\mathbf{w}-\mathbf{b} v)^{T} \Psi^{-1}(\mathbf{w}-\mathbf{b} v)+(\mathbf{t}-\Delta \mathbf{b} v)^{T} \Psi^{-1}(\mathbf{t}-\Delta \mathbf{b} v) .
$$

Sejam

$$
\begin{aligned}
& Q(v)=\left[v-\frac{(\mathbf{w}+\Delta \mathbf{t})^{T} \Psi^{-1} \mathbf{b}}{\mathbf{b}^{T}\left(\Psi^{-1}+\Delta \Psi^{-1} \Delta\right) \mathbf{b}}\right]^{2}, \\
& \mathrm{D}=\left[\Psi+\frac{\Delta \mathrm{bb}^{T} \Delta}{\mathrm{b}^{T}\left(\Psi^{-1}+\Delta \Psi^{-1} \Delta\right) \mathrm{b}}\right]^{-1}, \\
& \begin{aligned}
\mathrm{A} & =\Psi^{-1}-\frac{\Psi^{-1} \mathrm{bb}^{T} \Psi^{-1}}{\mathrm{~b}^{T}\left(\Psi^{-1}+\Delta \Psi^{-1} \Delta\right) \mathrm{b}} \\
\mathrm{d} & =\frac{\Psi^{-1} \Delta \mathrm{bb}^{T} \Psi^{-1}}{\mathrm{~b}^{\mathrm{T}}\left(\Psi^{-1}+\Delta \Psi^{-1} \Delta\right) \mathrm{bw}}
\end{aligned}
\end{aligned}
$$

Após algumas manipulações algébricas e usando a notação dada em (4.10), temos que,

$$
\begin{aligned}
& Q(v \mid \mathrm{w}, \mathrm{t})=\mathrm{w}^{T} \Psi^{-1} \mathbf{w}+2 \mathbf{b}^{T} \Psi^{-1} \mathbf{w} v+\mathbf{b}^{T} \Psi^{-1} \mathbf{b} v^{2}+\mathbf{t}^{T} \Psi^{-1} \mathbf{t}-2 \mathbf{b}^{T} \Delta \Psi^{-1} \mathbf{t} v+ \\
& +\mathrm{b}^{T} \Delta \Psi^{-1} \Delta \mathrm{b} v^{2} \\
& =\mathbf{b}^{T}\left(\Psi^{-1}+\Delta \Psi^{-1} \Delta\right) \mathbf{b} v^{2}-2 \mathbf{b}^{T} \Psi^{-1}(\mathbf{w}+\Delta \mathbf{t}) v+\mathbf{w}^{T} \Psi^{-1} \mathbf{w}+\mathbf{t}^{T} \Psi^{-1} \mathbf{t} \\
& =\mathrm{b}^{T}\left(\Psi^{-1}+\Delta \Psi^{-1} \Delta\right) \mathrm{b}\left[v-\frac{(\mathrm{w}+\Delta \mathrm{t})^{T} \Psi^{-1} \mathbf{b}}{\mathrm{b}^{T}\left(\Psi^{-1}+\Delta \Psi^{-1} \Delta\right) \mathbf{b}}\right]^{2}+\mathrm{w}^{T} \Psi^{-1} \mathrm{w}+\mathrm{t}^{T} \Psi^{-1} \mathbf{t}- \\
& -\frac{\left[(\mathrm{w}+\Delta \mathrm{t})^{T} \Psi^{-1} \mathrm{~b}\right]^{2}}{\mathrm{~b}^{T}\left(\Psi^{-1}+\Delta \Psi^{-1} \Delta\right) \mathrm{b}} \\
& =\mathrm{b}^{T}\left(\Psi^{-1}+\Delta \Psi^{-1} \Delta\right) \mathrm{b} Q(v)+\mathrm{w}^{T} \mathrm{Aw}+\mathrm{t}^{T} \mathrm{Dt}-2 \mathrm{~d}^{T} \mathrm{t} \\
& =\mathbf{b}^{T}\left(\Psi^{-1}+\Delta \Psi^{-1} \Delta\right) \mathbf{b} Q(v)+\mathrm{w}^{T} \mathrm{Aw}-\mathrm{d}^{T} \mathbf{D}^{-1} \mathrm{~d}+\left(\mathrm{t}-\mathbf{D}^{-1} \mathrm{~d}\right)^{T} \mathbf{D}\left(\mathbf{t}-\mathbf{D}^{-1} \mathrm{~d}\right) \\
& =\mathbf{b}^{T}\left(\Psi^{-1}+\Delta \Psi^{-1} \Delta\right) \mathbf{b} Q(v)+Q(\mathbf{w})+Q(\mathbf{t}) \text {. }
\end{aligned}
$$

Ozán, N. S. 
Assim,

$$
Q(v \mid \mathbf{w}, \mathbf{t})=\mathbf{b}^{T}\left(\Psi^{-1}+\Delta \Psi^{-1} \Delta\right) \mathbf{b} Q(v)+Q(\mathbf{w})+Q(\mathbf{t}),
$$

onde,

1. $Q(\mathrm{w})=\mathrm{w}^{T} \mathbf{A} \mathbf{w}-\mathbf{d}^{T} \mathbf{D}^{-1} \mathbf{d}$, que pode ser reescrito usando (4.10), resultando em,

$$
\begin{aligned}
& Q(\mathrm{w})=\mathrm{w}^{T}\left[\Psi^{-1}-\frac{\Psi^{-1} \mathbf{b b}^{T} \Psi^{-1}}{\mathrm{~b}^{T}\left(\Psi^{-1}+\Delta \Psi^{-1} \Delta\right) \mathbf{b}}\right] \mathrm{w}- \\
& -\mathrm{w}^{T} \frac{\Psi^{-1} \mathrm{bb}^{T} \Delta \Psi^{-1}}{\mathrm{~b}^{T}\left(\Psi^{-1}+\Delta \Psi^{-1} \Delta\right) \mathrm{b}}\left[\Psi+\frac{\Delta \mathrm{bb}^{T} \Delta}{\mathrm{b}^{T}\left(\Psi^{-1}+\Delta \Psi^{-1} \Delta\right) \mathrm{b}}\right]^{-1} \times \\
& \times\left[\frac{\Psi^{-1} \Delta \mathrm{bb}^{T} \Psi^{-1}}{\mathrm{~b}^{T}\left(\Psi^{-1}+\Delta \Psi^{-1} \Delta\right) \mathrm{b}} \mathrm{w}\right] \\
& =\mathrm{w}^{T}\left\{\Psi^{-1}-\left[1+\frac{\mathbf{b}^{T} \Delta \Psi^{-1} \Delta \mathrm{b}}{\mathrm{b}^{T}\left(\Psi^{-1}+\Delta \Psi^{-1} \Delta\right) \mathrm{b}}+\frac{\left(\mathrm{b}^{T} \Delta \Psi^{-1} \Delta \mathrm{b}\right)^{2}}{\left[\mathbf{b}^{T}\left(\Psi^{-1}+\Delta \Psi^{-1} \Delta\right) \mathrm{b}\right]\left(\mathbf{b}^{T} \Psi^{-1} \mathbf{b}\right)}\right] \times\right. \\
& \left.\times \frac{\Psi^{-1} \mathrm{bb}^{T} \Psi^{-1}}{\mathrm{~b}^{T}\left(\Psi^{-1}+\Delta \Psi^{-1} \Delta\right) \mathrm{b}}\right\} \mathrm{w} \\
& =\mathbf{w}^{T}\left\{\Psi^{-1}-\frac{1}{\mathbf{b}^{T} \Psi^{-1} \mathbf{b}} \Psi^{-1} \mathbf{b b}^{T} \Psi^{-1}\right\} \mathbf{w} \\
& =(\mathbf{z}-\boldsymbol{\mu})^{T} \Sigma^{-1}(\mathbf{z}-\boldsymbol{\mu}) \text {, }
\end{aligned}
$$

desde que

$$
\mathbf{w}=\left(\begin{array}{c}
0 \\
\mathbf{z}-\mu
\end{array}\right) \text { e } \boldsymbol{\Sigma}=\left(\begin{array}{cc}
\beta^{2} \sigma_{x}^{2}+\sigma_{e}^{2} & \beta \sigma_{x}^{2} \\
\beta \sigma_{x}^{2} & \sigma_{x}^{2}+\sigma_{u}^{2}
\end{array}\right)
$$

2. $Q(\mathrm{t})=\left(\mathrm{t}-\mathbf{D}^{-1} \mathbf{d}\right)^{T} \mathbf{D}\left(\mathbf{t}-\mathbf{D}^{-1} \mathbf{d}\right)$.

Logo a expressão de $f_{\mathbf{Z}}(\mathbf{z})$ em (4. 9) fica,

$$
\begin{aligned}
f_{\mathbf{Z}}(\mathrm{z}) & =\frac{2^{3}(2 \pi)^{-\frac{6}{2}}}{|\Psi|} \int_{\mathbf{t}<\Delta \mathrm{w}} \int_{-\infty}^{\infty} e^{-\frac{1}{2} Q(v \mid \mathbf{w}, \mathbf{t})} d v d \mathbf{t} \\
& =\frac{2^{3}(2 \pi)^{-\frac{6}{2}}}{|\Psi|} \int_{\mathbf{t}<\Delta \mathbf{w}} \int_{-\infty}^{\infty} e^{-\frac{1}{2}\left[\mathbf{b}^{T}\left(\Psi^{-1}+\Delta \Psi^{-1} \Delta\right) \mathbf{b} Q(v)+Q(\mathbf{w})+Q(\mathbf{t})\right]} d v d \mathbf{t} \\
& =\frac{2^{3}(2 \pi)^{-\frac{3}{2}}}{|\Psi|} e^{-\frac{1}{2} Q(\mathbf{w})} \int_{\mathbf{t}<\Delta \mathbf{w}}(2 \pi)^{-\frac{3}{2}} e^{-\frac{1}{2} Q(\mathrm{t})}\left[\int_{-\infty}^{\infty} \frac{e^{-\frac{1}{2}\left[\mathrm{~b}^{T}\left(\Psi^{-1}+\Delta \Psi^{-1} \Delta\right) \mathbf{b}\right] Q(v)}}{\sqrt{2 \pi}} d v\right] d \mathbf{t} \\
& =\frac{2^{3}(2 \pi)^{-\frac{3}{2}}}{|\Psi|\left[\mathbf{b}^{T}\left(\Psi^{-1}+\Delta \Psi^{-1} \Delta\right) \mathbf{b}\right]^{\frac{1}{2}}} e^{-\frac{1}{2} Q(\mathrm{w})} \int_{\mathbf{t}<\Delta \mathbf{w}}(2 \pi)^{-\frac{3}{2}} e^{-\frac{1}{2} Q(\mathrm{t})} d \mathbf{t} .
\end{aligned}
$$

Usando as expressões para $Q(\mathrm{w})$ e $Q(\mathrm{t})$ em (4.12), temos,

$$
f_{\mathbf{Z}}(\mathbf{z})=\frac{2^{3}(2 \pi)^{-1} e^{-\frac{1}{2}(\mathbf{z}-\mu)^{T} \Sigma^{-1}(\mathbf{z}-\boldsymbol{\mu})}}{|\Psi|\left[\mathbf{b}^{T}\left(\Psi^{-1}+\Delta \Psi^{-1} \Delta\right) \mathbf{b}\right]^{\frac{1}{2}}} \int_{t<\Delta w}(2 \pi)^{-\frac{3}{2}} e^{-\frac{1}{2}\left(\mathbf{t}-\mathbf{D}^{-1} \mathbf{d}\right) \mathbf{D}\left(\mathbf{t}-\mathbf{D}^{-1} \mathbf{d}\right)} d \mathbf{t} .
$$


Consideramos a seguir a transformação, $\mathrm{s}=\mathrm{D}^{-\frac{1}{2}}\left(\mathbf{t}-\mathbf{D}^{-1} \mathbf{d}\right)$, ou seja, $\mathbf{t}=\mathrm{D}^{-\frac{1}{2}} \mathbf{S}+\mathbf{D}^{-1} \mathrm{~d}$, com $d \mathbf{t}=|\mathbf{D}|^{-\frac{1}{2}} d \mathbf{s}$. Além disso, se $\mathbf{t}<\Delta \mathrm{w}$, então $\mathrm{s}<\mathrm{D}^{-\frac{1}{2}}\left(\Delta \mathrm{w}-\mathrm{D}^{-1} \mathrm{~d}\right)$, com $|\mathrm{D}|=|\Psi|^{-1} \frac{\mathrm{b}^{T} \Psi^{-1} \mathrm{~b}}{\mathrm{~b}^{T}\left(\Psi^{-1}+\Delta \Psi^{-1} \Delta\right) \mathrm{b}}$. Portanto,

$$
\begin{aligned}
\int_{t<\Delta \mathbf{w}}(2 \pi)^{-\frac{3}{2}} e^{-\frac{1}{2}\left(\mathbf{t}-\mathbf{D}^{-1} \mathbf{d}\right) \mathbf{D}\left(\mathbf{t}-\mathbf{D}^{-1} \mathbf{d}\right)} d \mathbf{t} & =|\Delta|^{-\frac{1}{2}} \int_{\mathbf{s}<\mathbf{D}^{\frac{1}{2}}\left(\Delta \mathbf{w}-\mathbf{D}^{-1} \mathbf{d}\right)}(2 \pi)^{-\frac{3}{2}} e^{-\frac{1}{2} \mathbf{s}^{T} \mathbf{s}} d \mathbf{s} \\
& =\Phi_{3}\left(\mathbf{D}^{-\frac{1}{2}}\left(\Delta \mathbf{w}-\mathbf{D}^{-1} \mathbf{d}\right)\right) \\
& =\Phi_{3}\left(\left(\Delta \mathbf{w}-\mathbf{D}^{-1} \mathbf{d}\right) \mid 0, \mathbf{D}^{-1}\right) .
\end{aligned}
$$

Notamos também que,

$$
\begin{aligned}
\frac{(2 \pi)^{-1} e^{-\frac{1}{2}(\mathbf{z}-\mu)^{T} \Sigma^{-1}(\mathbf{z}-\mu)}}{|\Psi|\left[\mathrm{b}^{T}\left(\Psi^{-1}+\Delta \Psi^{-1} \Delta\right) \mathbf{b}\right]^{\frac{1}{2}}} & =\frac{(2 \pi)^{-1} e^{-\frac{1}{2}(\mathbf{z}-\mu)^{T} \Sigma^{-1}(\mathbf{z}-\mu)}}{|\Sigma|^{\frac{1}{2}}} \\
& =\phi_{2}(\mathbf{z} \mid \boldsymbol{\mu}, \Sigma)
\end{aligned}
$$

Assim,

$$
f_{\mathbf{Z}}(\mathbf{z})=2^{3} \phi_{2}(\mathbf{z} \mid \mu, \Sigma) \Phi_{3}\left(\left(\Delta \mathbf{w}-\mathbf{D}^{-1} \mathbf{d}\right) \mid 0, \mathbf{D}^{-1}\right)
$$

Notando que,

$$
\begin{aligned}
\Delta \mathrm{w}-\mathrm{D}^{-1} \mathrm{~d} & =\Delta \mathrm{w}-\left[\Psi+\frac{\Delta \mathrm{bb}^{T} \Delta}{\mathrm{b}^{T} \Psi^{-1} \mathrm{~b}}\right] \frac{\Psi^{-1} \Delta \mathrm{bb}^{T} \Psi^{-1}}{\mathbf{b}^{T}\left(\Psi^{-1}+\Delta \Psi^{-1} \Delta\right) \mathrm{b}} \mathrm{w} \\
& =\Delta\left[\mathrm{I}_{3}-\frac{\mathrm{bb}^{T} \Psi^{-1}}{\mathrm{~b}^{T} \Psi^{-1} \mathbf{b}}\right] \mathbf{w} \\
& =\Delta \Psi \mathbf{B}^{T} \Sigma^{-1}(\mathrm{z}-\mu)
\end{aligned}
$$

podendo escrever,

$$
\begin{aligned}
\Phi_{3}\left(\left(\Delta \mathrm{w}-\mathrm{D}^{-1} \mathrm{~d}\right) \mid 0, \mathrm{D}^{-1}\right) & =\Phi_{3}\left(\Delta \Psi \mathrm{B}^{T} \Sigma^{-1}(\mathrm{z}-\mu) \mid 0,\left(\Psi+\frac{\Delta \mathrm{bb}^{T} \Delta}{\mathrm{b}^{T} \Psi^{-1} \mathrm{~b}}\right)\right) \\
& =\Phi_{3}\left(\Delta \Psi \mathrm{B}^{T} \Sigma^{-1}(\mathrm{z}-\mu) \mid \Delta \Psi \mathrm{B}^{T} \Sigma^{-1},\left(\Psi+\frac{\Delta \mathrm{bb}^{T} \Delta}{\mathrm{b}^{T} \Psi^{-1} \mathbf{b}}\right)\right) .
\end{aligned}
$$

Definido $\Delta_{*}=\Delta \Psi \mathrm{B}^{T} \Sigma^{-\frac{1}{2}}$ e com $\Sigma=\mathrm{B} \Psi \mathrm{B}^{T}$, então a expressão acima pode ser escrita como

$$
\Phi_{3}\left(\left(\Delta \mathrm{w}-\mathrm{D}^{-1} \mathrm{~d}\right) \mid 0, \mathrm{D}^{-1}\right)=\Phi_{3}\left(\Delta_{*} \Sigma^{-\frac{1}{2}}(\mathrm{z}-\mu) \mid 0,\left(\Psi+\frac{\Delta \mathrm{bb}^{T} \Delta}{\mathrm{b}^{T} \Psi^{-1} \mathrm{~b}}\right)\right) .
$$

Substituindo (4.15) em (4.14) fica provada o teorema.

No modelo normal assimétrico com erros nas variáveis podemos assumir que a suposição de assimetria está na covariável não observada $x_{i}$. Portanto o parâmetro de assimetria é $\lambda=\left(\lambda_{x}, \lambda_{e}, \lambda_{u}\right)=\left(\lambda_{x}, 0,0\right)$ onde $\lambda_{x} \neq 0$. Neste caso, é interessante ressaltar que a distribuição conjunta de $(Y, X)^{T}$ é normal assimétrica, apesar de os erros $e_{i}$ e $u_{i}$ serem normais simétrico. Assim temos o seguinte corolário. 
Corolario 4.2. Seja $\mathbf{Z}$ sob as suposições do Teorema 4.1., com $\mathbf{r}=(v, e, u)^{T} \stackrel{\text { ind }}{\sim} \operatorname{SNI}_{3}(0, \Psi, \lambda)$, onde $\Psi=\operatorname{diag}\left\{\sigma_{x}^{2}, \sigma_{e}^{2}, \sigma_{u}^{2}\right\}$ e $\lambda=\left(\lambda_{x}, 0,0\right)^{T}, \lambda_{x} \neq 0$, então a função de densidade para $\mathrm{Z}=(Y, X)^{T}$ é dada por

$$
f_{\mathbf{Z}}(\mathbf{z})=2|\Sigma|^{-\frac{1}{2}} \phi_{2}\left(\Sigma^{-\frac{1}{2}}(\mathbf{z}-\boldsymbol{\mu})\right) \Phi\left(\Delta_{* *} \boldsymbol{\Sigma}^{-\frac{1}{2}}(\mathbf{z}-\boldsymbol{\mu})\right)
$$

com,

$$
\text { - } \Delta_{* *}=d \mathrm{e}_{1} \mathrm{~b}_{1}^{T} \Sigma^{-\frac{1}{2}},
$$

- $d=\lambda_{x} \sigma_{x}^{2}\left(\sigma_{x}^{2}+\frac{\lambda_{x}^{2}}{\mathbf{b}^{T} \Psi^{-1} \mathbf{b}}\right)^{-\frac{1}{2}}, \mathbf{b}=(-1, \beta, 1)^{T}, \mathbf{b}_{1}=(\beta, 1)^{T}$ e $\mathbf{e}_{\mathbf{1}}=(1,0,0)^{T}$.

Demonstração. Neste caso temos que,

$$
x \sim S N\left(\mu_{x}, \sigma_{x}^{2}, \lambda_{x}\right), \quad e \sim S N\left(0, \sigma_{e}^{2}, 0\right), \quad u \sim S N\left(0, \sigma_{u}^{2}, 0\right),
$$

e portanto a função de densidade em (4.7) será modificada somente em $\Phi_{3}\left(\Delta_{*} \boldsymbol{\Sigma}^{-\frac{1}{2}}(\mathbf{z}-\boldsymbol{\mu})\right.$ ), com $\Delta_{*}=\left(\Psi+\frac{\Delta \mathbf{b b}^{T} \Delta}{\mathbf{b}^{T} \boldsymbol{\Psi}^{-1} \mathbf{b}}\right)^{-\frac{1}{2}} \Delta \boldsymbol{\Psi} \mathbf{B}^{T} \boldsymbol{\Sigma}^{-\frac{1}{2}}, \mathbf{b}=(-1, \beta, 1)^{T}$ e neste caso $\Delta=\operatorname{Diag}\left\{\lambda_{x}, 0,0\right\}$. Portanto, se

$$
\begin{aligned}
\Delta \mathbf{b b}^{T} \Delta & =\left(\begin{array}{ccc}
\lambda_{x} & 0 & 0 \\
0 & 0 & 0 \\
0 & 0 & 0
\end{array}\right)\left(\begin{array}{ccc}
1 & -\beta & -1 \\
-\beta & \beta^{2} & \beta \\
-1 & \beta & 1
\end{array}\right)\left(\begin{array}{ccc}
\lambda_{x} & 0 & 0 \\
0 & 0 & 0 \\
0 & 0 & 0
\end{array}\right)= \\
& =\left(\begin{array}{ccc}
\lambda_{x}^{2} & 0 & 0 \\
0 & 0 & 0 \\
0 & 0 & 0
\end{array}\right)=\lambda_{x}^{2}\left(\begin{array}{l}
1 \\
0 \\
0
\end{array}\right)\left(\begin{array}{lll}
1 & 0 & 0
\end{array}\right)=\lambda_{x}^{2} \mathbf{e}_{1} \mathbf{e}_{1}^{T},
\end{aligned}
$$

com $\mathbf{e}_{1}=(1,0,0)^{T}$, então,

$$
\begin{aligned}
& \Psi+\frac{\Delta \mathbf{b b}^{T} \Delta}{\mathbf{b}^{T} \Psi^{-1} \mathbf{b}}=\left(\begin{array}{ccc}
\sigma_{x}^{2} & 0 & 0 \\
0 & \sigma_{e}^{2} & 0 \\
0 & 0 & \sigma_{u}^{2}
\end{array}\right)+\frac{\lambda_{x}^{2}}{\mathbf{b}^{T} \Psi^{-1} \mathbf{b}}\left(\begin{array}{ccc}
1 & 0 & 0 \\
0 & 0 & 0 \\
0 & 0 & 0
\end{array}\right)= \\
& =\left(\begin{array}{ccc}
\sigma_{x}^{2}+\frac{\lambda_{x}^{2}}{\mathbf{b}^{T} \Psi^{-1} \mathbf{b}} & 0 & 0 \\
0 & \sigma_{e}^{2} & 0 \\
0 & 0 & \sigma_{u}^{2}
\end{array}\right) \text {. }
\end{aligned}
$$

Assim,

$$
\begin{aligned}
\Delta_{*} & =\left(\begin{array}{ccc}
\sigma_{x}^{2}+\frac{\lambda_{x}^{2}}{\mathbf{b}^{T} \Psi^{-1} \mathbf{b}} & 0 & 0 \\
0 & \sigma_{e}^{2} & 0 \\
0 & 0 & \sigma_{u}^{2}
\end{array}\right)^{-\frac{1}{2}}\left(\begin{array}{ccc}
\lambda_{x} \sigma_{x}^{2} & 0 & 0 \\
0 & 0 & 0 \\
0 & 0 & 0
\end{array}\right)\left(\begin{array}{cc}
\beta & 1 \\
1 & 0 \\
0 & 1
\end{array}\right) \Sigma^{-\frac{1}{2}}= \\
& =\left(\begin{array}{ccc}
\sigma_{x}^{2}+\frac{\lambda_{x}^{2}}{\mathbf{b}^{T} \Psi^{-1} \mathbf{b}} & 0 & 0 \\
0 & \sigma_{e}^{2} & 0 \\
0 & 0 & \sigma_{u}^{2}
\end{array}\right) \lambda_{x} \sigma_{x}^{2}\left(\begin{array}{cc}
\beta & 1 \\
0 & 0 \\
0 & 0
\end{array}\right) \Sigma^{-\frac{1}{2}}= \\
& =\lambda_{x} \sigma_{x}^{2}\left(\sigma_{x}^{2}+\frac{\lambda_{x}^{2}}{\mathbf{b}^{T} \Psi^{-1} \mathbf{b}}\right)^{-\frac{1}{2}}\left(\begin{array}{cc}
\beta & 1 \\
0 & 0 \\
0 & 0
\end{array}\right) \Sigma^{-\frac{1}{2}}
\end{aligned}
$$

Ozán, N. S. 
Definindo $d=\lambda_{x} \sigma_{x}^{2}\left(\sigma_{x}^{2}+\frac{\lambda_{x}^{2}}{\mathbf{b}^{T} \Psi^{-1} \mathbf{b}}\right)^{-\frac{1}{2}}, \mathbf{b}_{1}=(\beta, 1)^{T}$ e $\mathbf{e}_{1}=(1,0,0)^{T}$, temos que,

$$
\Delta_{*}=d \mathrm{e}_{1} \mathrm{~b}_{1}^{T} \Sigma^{-\frac{1}{2}}=\Delta_{* *} .
$$

Por outro lado,

$$
\begin{aligned}
\Delta_{* *} \Sigma^{-\frac{1}{2}}(\mathbf{z}-\boldsymbol{\mu}) & =d \mathbf{e}_{1} \mathbf{b}_{1} \boldsymbol{\Sigma}^{-1}(\mathbf{z}-\boldsymbol{\mu}) \\
& =d|\boldsymbol{\Sigma}|^{-1}\left(\begin{array}{cc}
\beta & 1 \\
0 & 0 \\
0 & 0
\end{array}\right)\left(\begin{array}{cc}
\sigma_{x}^{2}+\sigma_{u}^{2} & -\beta \sigma_{x}^{2} \\
-\beta \sigma_{x}^{2} & \beta^{2} \sigma_{x}^{2}+\sigma_{e}^{2}
\end{array}\right)\left(\begin{array}{c}
Y-\alpha-\beta \mu_{x} \\
X-\mu_{x}
\end{array}\right) \\
& =d|\Sigma|^{-1}\left(\begin{array}{cc}
\beta \sigma_{u}^{2} & \sigma_{e}^{2} \\
0 & 0 \\
0 & 0
\end{array}\right)\left(\begin{array}{c}
Y-\alpha-\beta \mu_{x} \\
X-\mu_{x}
\end{array}\right) \\
& =d|\Sigma|^{-1}\left(\begin{array}{c}
\beta \sigma_{u}^{2}\left(Y-\alpha-\beta \mu_{x}\right)+\sigma_{e}^{2}\left(X-\mu_{x}\right) \\
0 \\
0
\end{array}\right) \\
& =d|\Sigma|^{-1}\left[\beta \sigma_{u}^{2}\left(Y-\alpha-\beta \mu_{x}\right)+\sigma_{e}^{2}\left(X-\mu_{x}\right)\right]\left(\begin{array}{c}
1 \\
0 \\
0
\end{array}\right) \\
& =d|\Sigma|^{-1}\left[\beta \sigma_{u}^{2}\left(Y-\alpha-\beta \mu_{x}\right)+\sigma_{e}^{2}\left(X-\mu_{x}\right)\right] \mathbf{e}_{1},
\end{aligned}
$$

onde $\mathbf{e}_{1}=(1,0,0)^{T}$. Portanto ao resolver $\Phi_{3}\left(\Delta_{* *} \boldsymbol{\Sigma}^{-\frac{1}{2}}(\mathbf{z}-\boldsymbol{\mu})\right)$ temos que,

$$
\Phi_{3}\left(\Delta_{* *} \Sigma^{-\frac{1}{2}}(\mathbf{z}-\boldsymbol{\mu})\right)=\frac{1}{2^{2}} \Phi\left(\Delta_{* *} \Sigma^{-\frac{1}{2}}(\mathbf{z}-\boldsymbol{\mu})\right) .
$$

Fica assim provado o corolário.

Observação 8. Desde que,

$$
\begin{aligned}
\Phi_{3}\left(\Delta_{*} \Sigma^{-\frac{1}{2}}(\mathbf{z}-\boldsymbol{\mu})\right) & =\Phi_{3}\left(\Delta_{* *} \Sigma^{-\frac{1}{2}}(\mathbf{z}-\boldsymbol{\mu})\right) \\
& =\Phi_{3}\left(d|\Sigma|^{-1}\left[\beta \sigma_{u}^{2}\left(Y-\alpha-\beta \mu_{x}\right)+\sigma_{e}^{2}\left(X-\mu_{x}\right)\right] \mathbf{e}_{1}\right) \\
& =\frac{1}{2^{2}} \Phi\left(d|\Sigma|^{-1} \beta \sigma_{u}^{2}\left(Y-\alpha-\beta \mu_{x}\right)+\sigma_{e}^{2}\left(X-\mu_{x}\right)\right)
\end{aligned}
$$

a expressão (4.16) pode ser escrita como:

$$
f_{Z}(\mathbf{z})=2|\Sigma|^{-\frac{1}{2}} \phi_{2}\left(\Sigma^{-\frac{1}{2}}(\mathbf{z}-\mu)\right) \Phi\left(d|\Sigma|^{-1}\left[\beta \sigma_{u}^{2}\left(y-\alpha-\beta \mu_{x}\right)+\sigma_{e}^{2}\left(x-\mu_{x}\right)\right]\right) .
$$

Seja $\mathbf{Z}_{1}, \ldots, \mathbf{Z}_{n}$ uma amostra aleatória de $n$ observações independentes sob as suposições do Teorema 3.1. Então a função de densidade marginal de $\mathbf{Z}_{i}=\left(Y_{i}, X_{i}\right)^{T}, i=1, \ldots, n$, é

$$
f_{Z}\left(\mathbf{z}_{i} \mid \theta, \lambda\right)=2^{3}|\Sigma|^{-\frac{1}{2}} \phi_{2}\left(\Sigma^{-\frac{1}{2}}\left(\mathbf{z}_{i}-\mu\right)\right) \Phi_{3}\left(\Delta_{*} \Sigma^{-\frac{1}{2}}\left(\mathbf{z}_{i}-\mu\right)\right),
$$

Ozán, N. S. 
$\operatorname{com} \theta=\left(\alpha, \beta, \mu_{x}, \sigma_{x}^{2}, \sigma_{e}^{2}, \sigma_{u}^{2}\right)^{T}$ e $\lambda=\left(\lambda_{x}, \lambda_{e}, \lambda_{u}\right)^{T}$.

A função de verossimilhança para uma amostra independente de tamanho $n$, é

$$
L\left(\boldsymbol{\theta}, \lambda \mid \mathrm{z}_{1}, \ldots, \mathbf{z}_{n}\right)=\prod_{i=n}^{n} f_{Z}\left(\mathbf{z}_{i} \mid \boldsymbol{\theta}, \boldsymbol{\lambda}\right),
$$

onde $f_{Z}\left(\mathbf{z}_{i} \mid \theta, \lambda\right)$, é a densidade marginal em (4.18).

Observação 9. O problema de falta de identificabilidade do modelo simétrico também observa-se no modelo assimétrico. Um modelo é identificável se para dois valores diferentes do vector de parâmetros do modelo temos diferentes valores da função de densidade. Considerando o vector de parâmetros $\theta^{*}=(\theta, \lambda)=\left(\alpha, \beta, \mu_{x}, \sigma_{x}^{2}, \sigma_{e}^{2}, \sigma_{u}^{2}, \lambda_{x}, \lambda_{e}, \lambda_{u}\right)^{T}$ e a função de densidade $f_{\mathbf{Z}}\left(\mathbf{z} \mid \boldsymbol{\theta}^{*}\right)$ dada na equação (3.9), o modelo é identificável se $\boldsymbol{\theta}_{1}^{*} \neq \boldsymbol{\theta}_{2}^{*}$ então $f_{\mathbf{Z}}\left(\mathbf{z} \mid \boldsymbol{\theta}_{1}^{*}\right) \neq f_{\mathbf{Z}}\left(\mathbf{z} \mid \boldsymbol{\theta}_{2}^{*}\right)$.

Sejam $\boldsymbol{\theta}_{1}^{*}=(1,1,1,1,1,1,0,0,0)$ e $\boldsymbol{\theta}_{2}^{*}=(1.5,0.5,1,2,1.5,0,0,0,0)$, de modo que $\boldsymbol{\theta}_{1}^{*} \neq \boldsymbol{\theta}_{2}^{*}$. Assim, $\boldsymbol{\mu}\left(\theta_{1}^{*}\right)=\boldsymbol{\mu}\left(\theta_{2}^{*}\right)=(2,1)^{T}, \boldsymbol{\Sigma}\left(\theta_{1}^{*}\right)=\Sigma\left(\theta_{2}^{*}\right)=\left(\begin{array}{cc}2 & 1 \\ 1 & 2\end{array}\right)$ e $\Delta\left(\theta_{1}^{*}\right)=\Delta\left(\theta_{2}^{*}\right)=0$, tal . que, $f_{Z}\left(\mathbf{z} \mid \boldsymbol{\theta}_{1}^{*}\right)=f_{Z}\left(\mathbf{z} \mid \boldsymbol{\theta}_{2}^{*}\right)$, e o modelo é não-identificável.

A solução proposta na literatura para o modelo elíptico, que apresentam o mesmo problemas de não identificabilidade, é considerar conhecida uma das variâncias $\sigma_{u}^{2}\left(\sigma_{e}^{2}\right)$, a razão de variâncias $\frac{\sigma_{e}^{2}}{\sigma_{u}^{2}}$ ou o coeficiente de confiabilidade $\frac{\sigma_{x}^{2}}{\sigma_{x}^{2}+\sigma_{u}^{2}}$.

\subsubsection{Um resultado de invariância para Inferência Bayesiana}

Nesta seção apresentamos um resultado de invariância para inferência Bayesiana considerando uma classe especial de distribuições a priori e uma extensão do modelo considerado nas seções anteriores. Enfatizamos que a invariância a que nos referimos está ligada a validade da inferência obtida sob o modelo normal.

Consideramos, as seguintes suposições no modelo (4.4), sustituindo (1.5) por,

$$
v_{i} \stackrel{i i d}{\sim} S N\left(0, \sigma_{x}^{2}, \lambda_{x i}\right), \quad e_{i} \stackrel{i i d}{\sim} S N\left(0, \sigma_{e}^{2}, \lambda_{e i}\right), \quad u_{i} \stackrel{i i d}{\sim} S N\left(0, \sigma_{u}^{2}, \lambda_{u i}\right)
$$

respectivamente. Além disso, $\lambda_{i}=\left(\lambda_{x i}, \lambda_{e i}, \lambda_{u i}\right)^{T}, i=1, \ldots, n$, são independentes e identicamente distribuidos com distribuição a priori assimétrica, ou seja,

$$
\lambda_{x i} \stackrel{i i d}{\sim} S N\left(0,1, w_{x}\right), \quad \lambda_{e i} \stackrel{i i d}{\sim} S N\left(0,1, w_{e}\right), \quad \lambda_{u i} \stackrel{i i d}{\sim} S N\left(0,1, w_{u}\right)
$$

e considerando as suposições de identificabilidade dada por $\sigma_{e}^{2}=\sigma_{u}^{2}=\sigma^{2}$. Assim, o parâmetro de interesse é $\boldsymbol{\theta}=\left(\alpha, \beta, \mu_{x}, \sigma_{x}^{2}, \sigma^{2}\right)$.

A densidade a priori para $(\theta, \lambda)$, onde $\lambda=\left(\lambda_{1}^{T}, \ldots, \lambda_{n}^{T}\right)^{T}$, é

$$
\begin{aligned}
p(\boldsymbol{\theta}, \boldsymbol{\lambda}) & =p(\boldsymbol{\theta}) \prod_{i=1}^{n} p\left(\boldsymbol{\lambda}_{i}\right) \\
& =p(\boldsymbol{\theta}) \prod_{i=1}^{n} p\left(\lambda_{x i}\right) p\left(\lambda_{e i}\right) p\left(\lambda_{u i}\right),
\end{aligned}
$$

Ozán, N. S. 
com $p\left(\lambda_{x i}\right)=2 \phi\left(\lambda_{x i}\right) \Phi\left(w_{x} \lambda_{x i}\right)$ e similarmente para $p\left(\lambda_{e i}\right)$ e $p\left(\lambda_{u i}\right)$, para $i=1, \ldots, n$. Portanto, temos o seguinte resultado.

Proposição 4.3. Sob o modelo (4.1) com as suposições (4.19) e (4.20), a inferência Bayesiana para $\theta$ se reduz a inferência Bayesiana sob o modelo normal usual com $\sigma_{e}^{2}=\sigma_{u}^{2}$ (Lindley e El Sayad. 1969).

Demonstração. Seja $\lambda=\left(\lambda_{1}^{T}, \ldots, \lambda_{n}^{T}\right)^{T}$, tal que $\lambda_{i}=\left(\lambda_{x i}, \lambda_{e i}, \lambda_{u i}\right), i=1, \ldots, n$, e com $\lambda_{i} \stackrel{i i d}{\sim} S N I_{3}(\mathbf{0}, \mathbf{I}, \mathbf{W})$ com $\mathbf{W}=\operatorname{diag}\left\{w_{x}, w_{e}, w_{u}\right\}$. Portanto, temos que,

$$
p\left(\boldsymbol{\lambda}_{i}\right)=p\left(\lambda_{x i}, \lambda_{e i}, \lambda_{u i}\right)=2^{3} \phi\left(\lambda_{x i}\right) \Phi\left(w_{x} \lambda_{x i}\right) \phi\left(\lambda_{e i}\right) \Phi\left(w_{e} \lambda_{e i}\right) \phi\left(\lambda_{u i}\right) \Phi\left(w_{u} \lambda_{u i}\right),
$$

com $i=1, \ldots, n$.

Considerando $\boldsymbol{\theta}=\left(\alpha, \beta, \mu_{x}, \sigma_{x}^{2}, \sigma\right)^{2}$ e $\lambda=\left(\lambda_{1}^{T}, \ldots, \lambda_{n}^{T}\right)^{T}$, independentes, então a função de densidade conjunta a priori de $(\boldsymbol{\theta}, \boldsymbol{\lambda})$ é

$$
p(\boldsymbol{\theta}, \boldsymbol{\lambda})=p(\boldsymbol{\theta}) p(\boldsymbol{\lambda}) .
$$

A função de distribuição a posteriori de $(\boldsymbol{\theta}, \boldsymbol{\lambda})$ dada uma amostra $\mathbf{z}_{1} \ldots, \mathbf{z}_{n}$ é,

$$
p\left(\boldsymbol{\theta}, \boldsymbol{\lambda} \mid \mathbf{z}_{1} \ldots, \mathbf{z}_{n}\right) \propto L\left(\mathbf{z}_{1} \ldots, \mathbf{z}_{n} \mid \boldsymbol{\theta}, \boldsymbol{\lambda}\right) p(\boldsymbol{\theta}, \boldsymbol{\lambda}),
$$

de modo que a distribuição a posteriori marginal de $\theta$ é,

$$
\begin{aligned}
p\left(\boldsymbol{\theta} \mid \mathbf{z}_{1} \ldots, \mathbf{z}_{n}\right) & \propto \int_{\mathrm{R}^{n}} L\left(\mathbf{z}_{1} \ldots, \mathbf{z}_{n} \mid \boldsymbol{\theta}, \boldsymbol{\lambda}\right) p(\boldsymbol{\theta}, \boldsymbol{\lambda}) d \boldsymbol{\lambda} \\
& =\int_{\mathrm{R}^{n}}\left[\prod_{i=1}^{n} f_{Z}\left(\mathbf{z}_{i} \mid \boldsymbol{\theta}, \boldsymbol{\lambda}_{i}\right) p(\boldsymbol{\theta}) p\left(\boldsymbol{\lambda}_{i}\right)\right] d \boldsymbol{\lambda}_{i} \\
& =p(\boldsymbol{\theta}) \prod_{i=1}^{n} \int_{\mathrm{R}^{3}} f_{Z}\left(\mathbf{z}_{i} \mid \boldsymbol{\theta}, \boldsymbol{\lambda}_{i}\right) p\left(\boldsymbol{\lambda}_{i}\right) d \boldsymbol{\lambda}_{i} .
\end{aligned}
$$

Por outro lado, para um particular $\mathbf{z}_{i}$, temos que,

$$
\begin{aligned}
& f_{Z}\left(\mathbf{z}_{i} \mid \theta, \lambda_{i}\right)= \int_{\mathrm{R}} f\left(Y_{i}, X_{i} \mid x_{i}\right) f\left(x_{i}\right) d x_{i} \\
&= \int_{\mathrm{R}} \frac{2^{3}}{\sigma_{x} \sigma_{e} \sigma_{u}} \phi\left(\frac{Y_{i}-\alpha-\beta x_{i}}{\sigma_{e}}\right) \phi\left(\frac{X_{i}-x_{i}}{\sigma_{u}}\right) \phi\left(\frac{x_{i}-\mu_{x}}{\sigma_{x}}\right) \\
& \Phi\left(\lambda_{e i} \frac{Y_{i}-\alpha-\beta x_{i}}{\sigma_{e}}\right) \Phi\left(\lambda_{u i} \frac{X_{i}-x_{i}}{\sigma_{u}}\right) \Phi\left(\lambda_{x i} \frac{x_{i}-\mu_{x}}{\sigma_{x}}\right) d x_{i},
\end{aligned}
$$

de modo que, para $i=1, \ldots, n$,

$$
\begin{aligned}
& \int_{\mathrm{R}^{3}} f_{Z}\left(\mathbf{z}_{i} \mid \theta, \lambda_{i}\right) p\left(\boldsymbol{\lambda}_{i}\right) d \boldsymbol{\lambda}_{i}= \int_{\mathrm{R}^{3}}\left[\int_{\mathrm{R}} \begin{array}{l}
\frac{2^{3}}{\sigma_{x} \sigma_{e} \sigma_{u}} \phi\left(\frac{Y_{i}-\alpha-\beta x_{i}}{\sigma_{e}}\right) \phi\left(\frac{X_{i}-x_{i}}{\sigma_{u}}\right) \phi\left(\frac{x_{i}-\mu_{x}}{\sigma_{x}}\right) \\
\left.\Phi\left(\lambda_{e i} \frac{Y_{i}-\alpha-\beta x_{i}}{\sigma_{e}}\right) \Phi\left(\lambda_{u i} \frac{X_{i}-x_{i}}{\sigma_{u}}\right) \Phi\left(\lambda_{x i} \frac{x_{i}-\mu_{x}}{\sigma_{x}}\right) d x_{i}\right] p\left(\lambda_{i}\right) d \lambda_{i}
\end{array}\right. \\
&=\int_{\mathrm{R}} \begin{array}{l}
\frac{2^{3}}{\sigma_{x} \sigma_{e} \sigma_{u}} \phi\left(\frac{Y_{i}-\alpha-\beta x_{i}}{\sigma_{e}}\right) \phi\left(\frac{X_{i}-x_{i}}{\sigma_{u}}\right) \phi\left(\frac{x_{i}-\mu_{x}}{\sigma_{x}}\right) \\
{\left[\int_{\mathrm{R}^{3}} \Phi\left(\lambda_{e i} \frac{Y_{i}-\alpha-\beta x_{i}}{\sigma_{e}}\right) \Phi\left(\lambda_{u i} \frac{X_{i}-x_{i}}{\sigma_{u}}\right) \Phi\left(\lambda_{x i} \frac{x_{i}-\mu_{x}}{\sigma_{x}}\right) p\left(\boldsymbol{\lambda}_{i}\right) d \boldsymbol{\lambda}_{i}\right] d x_{i} .}
\end{array}
\end{aligned}
$$

Ozán, N. S. 
Analisando a expressão acima, temos que,

$$
\begin{gathered}
\int_{\mathrm{R}^{3}} \Phi\left(\lambda_{e i} \frac{Y_{i}-\alpha-\beta x_{i}}{\sigma_{e}}\right) \Phi\left(\lambda_{u i} \frac{X_{i}-x_{i}}{\sigma_{u}}\right) \Phi\left(\lambda_{x i} \frac{x_{i}-\mu_{x}}{\sigma_{x}}\right) p\left(\lambda_{i}\right) d \lambda_{i}= \\
=\int_{\mathrm{R}^{3}} \begin{array}{l}
\Phi\left(\lambda_{e i} \frac{Y_{i}-\alpha-\beta x_{i}}{\sigma_{e}}\right) \Phi\left(\lambda_{u i} \frac{X_{i}-x_{i}}{\sigma_{u}}\right) \Phi\left(\lambda_{x i} \frac{x_{i}-\mu_{x}}{\sigma_{x}}\right) \\
{\left[2^{3} \phi\left(\lambda_{x i}\right) \Phi\left(w_{x} \lambda_{x i}\right) \phi\left(\lambda_{e i}\right) \Phi\left(w_{e} \lambda_{e i}\right) \phi\left(\lambda_{u i}\right) \Phi\left(w_{u} \lambda_{u i}\right)\right] d \lambda_{i},}
\end{array}
\end{gathered}
$$

onde,

$$
\int_{\mathrm{R}} \Phi\left(\lambda_{e i} \frac{Y_{i}-\alpha-\beta x_{i}}{\sigma_{e}}\right) \phi\left(\lambda_{e i}\right) \Phi\left(w_{e} \lambda_{e i}\right) d \lambda_{e i}=\frac{1}{2},
$$

e analogamente para as integrais com respeito a $d \lambda_{u i}$ e $d \lambda_{x i}$. Logo,

$$
\begin{aligned}
\int_{\mathrm{R}^{3}} f_{Z}\left(\mathbf{z}_{i} \mid \boldsymbol{\theta}, \lambda_{i}\right) p\left(\boldsymbol{\lambda}_{i}\right) d \boldsymbol{\lambda}_{i} & =\int_{\mathrm{R}} \frac{2^{3}}{\sigma_{x} \sigma_{e} \sigma_{u}} \phi\left(\frac{Y_{i}-\alpha-\beta x_{i}}{\sigma_{e}}\right) \phi\left(\frac{X_{i}-x_{i}}{\sigma_{u}}\right) \phi\left(\frac{x_{i}-\mu_{x}}{\sigma_{x}}\right) d x_{i} \\
& =L^{*}\left(\mathbf{z}_{i} \mid \boldsymbol{\theta}\right)
\end{aligned}
$$

como $L^{*}\left(\mathbf{z}_{1} \ldots, \mathbf{z}_{n} \mid \boldsymbol{\theta}\right)=\prod_{i=1}^{n} L^{*}\left(\mathbf{z}_{i} \mid \boldsymbol{\theta}\right)$ é a função de verossimilhança sob o modelo normal, portanto,

$$
p\left(\boldsymbol{\theta} \mid \mathbf{z}_{1} \ldots, \mathbf{z}_{n}\right) \propto L^{*}\left(\mathbf{z}_{1}, \ldots, \mathbf{z}_{n} \mid \boldsymbol{\theta}\right) p(\boldsymbol{\theta}),
$$

ficando assim provada a proposição.

Note que a distribuição a posteriori em (4.23) é independente de $\lambda$, ou seja, é a mesma que sob o modelo normal usual. Assim, resultados em Lindley e El Sayad (1969), Bolfarine e Cordani (1993), entre outros, valem também para o modelo normal assimétrico.

\subsection{Distribuição assintótica conjunta de $\left(\overline{\mathrm{Z}}, \mathrm{S}_{z}\right)$}

Sejam $\mathbf{Z}_{1}, \mathbf{Z}_{2}, \ldots, \mathbf{Z}_{n}$, independentes segundo a especificação da Seção 4.2.

$\mathrm{O}$ vetor de médias da amostra é $\overline{\mathrm{Z}}=\frac{1}{n} \sum_{i=1}^{n} \mathrm{Z}_{i}$ e a matriz do covariâncias amostrais é $\mathrm{S}_{Z}=\frac{1}{n} \sum_{i=1}^{n}\left(\mathbf{Z}_{i}-\overline{\mathbf{Z}}\right)\left(\mathrm{Z}_{i}-\overline{\mathbf{Z}}\right)^{T}$. Apresentamos a seguir alguns resultados importantes para auxiliam na derivação da distribuição conjunta de $\left(\bar{Z}, S_{Z}\right)$.

Ozán, N. S.

IME/USP 
Proposição 4.4. Seja $\mathbf{r}_{i}=\left(v_{i}, e_{i}, u_{i}\right)^{T}$, tal que $\mathbf{r}_{i} \stackrel{i i d}{\sim} S N I_{3}(0, \Psi, \lambda)$ para $i=1, \ldots, n$, onde $\boldsymbol{\Psi}=\operatorname{diag}\left\{\sigma_{x}^{2}, \sigma_{e}^{2}, \sigma_{u}^{2}\right\}$ e $\boldsymbol{\lambda}=\left(\lambda_{x}, \lambda_{e}, \lambda_{u}\right)^{T}$. Então,

$$
E\left(\mathrm{r}_{i}\right)=\sqrt{\frac{2}{\pi}} \Psi^{\frac{1}{2}} \delta \quad e \quad \operatorname{Var}\left(\mathrm{r}_{i}\right)=\left[\mathbf{I}_{3}-\frac{2}{\pi} \mathbf{D}^{2}(\delta)\right] \Psi,
$$

$\operatorname{com} \Psi^{\frac{1}{2}}=\operatorname{diag}\left\{\sigma_{x}, \sigma_{e}, \sigma_{u}\right\}, \delta=\left(\delta_{x}, \delta_{e}, \delta_{u}\right)^{T}, \mathbf{D}^{2}(\delta)=\operatorname{diag}\left\{\delta_{x}^{2}, \delta_{e}^{2}, \delta_{u}^{2}\right\}, \delta_{x}=\frac{\lambda_{x}}{\sqrt{1+\lambda_{x}^{2}}}$, $\delta_{e}=\frac{\lambda_{e}}{\sqrt{1+\lambda_{e}^{2}}}, \delta_{u}=\frac{\lambda_{u}}{\sqrt{1+\lambda_{u}^{2}}}$ e $\mathbf{I}_{3}$ a matriz identidade de dimensão 3.

Demonstração. Se $\mathrm{r}_{i} \stackrel{i i d}{\sim} S N I_{3}(0, \Psi, \lambda)$, então $v_{i} \stackrel{i i d}{\sim} S N\left(0, \sigma_{x}^{2} \lambda_{x}\right), e_{i} \stackrel{i i d}{\sim} S N\left(0, \sigma_{e}^{2}, \lambda_{e}\right) \mathrm{e}$ $u_{i} \stackrel{i i d}{\sim} S N\left(0, \sigma_{u}^{2}, \lambda_{u}\right)$, de onde segue que $\frac{v_{i}}{\sigma_{x}} \stackrel{i i d}{\sim} S N\left(\lambda_{x}\right)$,

$\frac{e_{i}}{\sigma_{e}} \stackrel{i i d}{\sim} S N\left(\lambda_{e}\right)$ e $\frac{u_{i}}{\sigma_{u}} \stackrel{i i d}{\sim} S N\left(\lambda_{u}\right), i=1, \ldots, n$

Assim, usando os resultados (2.16) e (2.17), temos que,

$$
\begin{array}{lll}
E\left(v_{i}\right)=\sqrt{\frac{2}{\pi}} \sigma_{x} \delta_{x} & E\left(e_{i}\right)=\sqrt{\frac{2}{\pi}} \sigma_{e} \delta_{u} & E\left(u_{i}\right)=\sqrt{\frac{2}{\pi}} \sigma_{u} \delta_{u} \\
E\left(v_{i}^{2}\right)=\sigma_{x}^{2} & E\left(e_{i}^{2}\right)=\sigma_{e}^{2} & E\left(u_{i}^{2}\right)=\sigma_{u}^{2} \\
E\left(v_{i}^{3}\right)=\sqrt{\frac{2}{\pi}} \sigma_{x}^{3} \delta_{x}\left(3-\delta_{x}^{2}\right) & E\left(e_{i}^{3}\right)=\sqrt{\frac{2}{\pi}} \sigma_{e}^{3} \delta_{e}\left(3-\delta_{e}^{2}\right) & E\left(u_{i}^{3}\right)=\sqrt{\frac{2}{\pi}} \sigma_{u}^{3} \delta_{u}\left(3-\delta_{u}^{2}\right) \\
E\left(v_{i}^{4}\right)=3 \sigma_{x}^{4} & E\left(e_{i}^{4}\right)=3 \sigma_{e}^{4} & E\left(u_{i}^{4}\right)=3 \sigma_{u}^{4} .
\end{array}
$$

Portanto,

$$
E\left(\mathrm{r}_{i}\right)=\left(\begin{array}{c}
\sqrt{\frac{2}{\pi}} \sigma_{x} \delta_{x} \\
\sqrt{\frac{2}{\pi}} \sigma_{e} \delta_{e} \\
\sqrt{\frac{2}{\pi}} \sigma_{u} \delta_{u}
\end{array}\right)=\sqrt{\frac{2}{\pi}}\left(\begin{array}{ccc}
\sigma_{x} & 0 & 0 \\
0 & \sigma_{e} & 0 \\
0 & 0 & \sigma_{u}
\end{array}\right)\left(\begin{array}{c}
\delta_{x} \\
\delta_{e} \\
\delta_{u}
\end{array}\right)
$$

de modo que $E\left(\mathbf{r}_{i}\right)=\sqrt{\frac{2}{\pi}} \Psi^{\frac{1}{2}} \delta$, com $\Psi^{\frac{1}{2}}=\operatorname{diag}\left\{\sigma_{x}, \sigma_{e}, \sigma_{u}\right\}$ e $\delta=\left(\delta_{x}, \delta_{e}, \delta_{u}\right)^{T}$, o que prova a primeira parte em (4.22). Para completar a prova usando as equações (4.8), calculamos a variância, $\operatorname{Var}\left(\mathbf{r}_{i}\right)=\operatorname{diag}\left\{\operatorname{Var}\left(v_{i}\right), \operatorname{Var}\left(e_{i}\right), \operatorname{Var}\left(u_{i}\right)\right\}, i=1, \ldots, n$.

Notando que,

$$
\begin{aligned}
& \operatorname{Var}\left(v_{i}\right)=\sigma_{x}^{2}-\frac{2}{\pi} \delta_{x}^{2} \sigma_{x}^{2}=\sigma_{x}^{2}\left(1-\frac{2}{\pi} \delta_{x}^{2}\right) \\
& \operatorname{Var}\left(e_{i}\right)=\sigma_{e}^{2}-\frac{2}{\pi} \delta_{e}^{2} \sigma_{e}^{2}=\sigma_{e}^{2}\left(1-\frac{2}{\pi} \delta_{e}^{2}\right) \\
& \operatorname{Var}\left(u_{i}\right)=\sigma_{u}^{2}-\frac{2}{\pi} \delta_{u}^{2} \sigma_{u}^{2}=\sigma_{u}^{2}\left(1-\frac{2}{\pi} \delta_{u}^{2}\right),
\end{aligned}
$$

temos,

$$
\operatorname{Var}\left(\mathbf{r}_{i}\right)=\left(\begin{array}{ccc}
\sigma_{x}^{2}\left(1-\frac{2}{\pi} \delta_{x}^{2}\right) & 0 & 0 \\
0 & \sigma_{e}^{2}\left(1-\frac{2}{\pi} \delta_{e}^{2}\right) & 0 \\
0 & 0 & \sigma_{u}^{2}\left(1-\frac{2}{\pi} \delta_{u}^{2}\right)
\end{array}\right)=\left(I_{3}-\frac{2}{\pi} D^{2}(\delta)\right) \Psi
$$

$\operatorname{com} \mathbf{D}^{2}(\delta)=\operatorname{diag}\left\{\delta_{x}^{2}, \delta_{e}^{2}, \delta_{u}^{2}\right\}$ 
Lema 4.5. Sendo $\mathbf{Z}_{1}, \mathbf{Z}_{2}, \ldots, \mathbf{Z}_{n}$, independentes, sob as suposições consideradas acima, temos que,

i. $E\left(\mathbf{Z}_{i}\right)=\boldsymbol{\mu}_{Z}=\boldsymbol{\mu}+\boldsymbol{\mu}_{\delta} \operatorname{com} \boldsymbol{\mu}=\left(\alpha+\beta \mu_{x}, \mu_{x}\right)^{T}$ e $\boldsymbol{\mu}_{\delta}=\sqrt{\frac{2}{\pi}} \mathbf{B} \Psi^{\frac{1}{2}} \boldsymbol{\delta}$, tal que, $\boldsymbol{\Psi}^{\frac{1}{2}}=\operatorname{diag}\left\{\sigma_{x}, \sigma_{e}, \sigma_{u}\right\}$ e $\delta=\left(\delta_{x}, \delta_{e}, \delta_{u}\right)^{T}$.

ii. $\operatorname{Var}\left(\mathbf{Z}_{i}\right)=\boldsymbol{\Sigma}_{Z}=\boldsymbol{\Sigma}-\boldsymbol{\Sigma}_{\delta} \operatorname{com} \boldsymbol{\Sigma}=\mathbf{B} \boldsymbol{\Psi} \mathbf{B}^{T}$ e $\boldsymbol{\Sigma}_{\delta}=\frac{2}{\pi} \mathbf{B D}(\delta) \Psi D(\delta) \mathbf{B}^{T}$, tal que, $\Psi=\operatorname{diag}\left\{\sigma_{x}^{2}, \sigma_{e}^{2}, \sigma_{u}^{2}\right\}$ e $\mathbf{D}(\boldsymbol{\delta})=\operatorname{diag}\left\{\delta_{x}, \delta_{e}, \delta_{u}\right\}$.

Demonstração. i. Se $\mathbf{Z}_{i}=\boldsymbol{\mu}+\mathbf{B r}_{i}$ então $E\left(\mathbf{Z}_{i}\right)=\boldsymbol{\mu}+\mathbf{B} E\left(\mathbf{r}_{i}\right)$ e pela Proposição 4.4, $E\left(\mathrm{Z}_{i}\right)=\mu+\mathrm{B} \sqrt{\frac{2}{\pi}} \Psi^{\frac{1}{2}} \delta$.

Assim se $E\left(\mathbf{Z}_{i}\right)=\mu_{Z}$ e $\boldsymbol{\mu}_{\delta}=\sqrt{\frac{2}{\pi}} \mathrm{B} \Psi^{\frac{1}{2}} \delta$, segue o resultado (i).

ii. Se $\mathbf{Z}_{i}=\boldsymbol{\mu}+\mathbf{B r}_{i}$, então, $\operatorname{Var}\left(\mathbf{Z}_{i}\right)=\mathbf{B} \operatorname{Var}\left(\mathbf{r}_{i}\right) \mathbf{B}^{T}$ e pela Proposição 4.4, $\operatorname{Var}\left(\mathbf{Z}_{i}\right)=\mathbf{B}\left[\mathbf{I}_{3}-\frac{2}{\pi} \mathbf{D}^{2}(\delta)\right] \Psi \mathbf{B}^{T}=\mathbf{B} \Psi \mathbf{B}^{T}-\frac{2}{\pi} \mathbf{B} D(\delta) \Psi \mathbf{D}(\delta) \mathbf{B}^{T}$.

Assim se $\operatorname{Var}\left(\mathbf{Z}_{i}\right) \stackrel{\pi}{=} \Sigma_{Z}, \Sigma=\mathrm{B} \Psi \mathrm{B}^{T}$ e $\Sigma_{\delta}=\frac{2}{\pi} \mathrm{BD}(\delta) \Psi \mathrm{D}(\delta) \mathrm{B}^{T}$, temos o resultade (ii).

Observação 10. Depois de algumas manipulações algébricas segue que,

$$
\Sigma=\left(\begin{array}{cc}
\beta^{2} \sigma_{x}^{2}+\sigma_{e}^{2} & \beta \sigma_{x}^{2} \\
\beta \sigma_{x}^{2} & \sigma_{x}^{2}+\sigma_{u}^{2}
\end{array}\right)
$$

e

$$
\Sigma_{\delta}=\frac{2}{\pi}\left(\begin{array}{cc}
\beta^{2} \sigma_{x}^{2} \delta_{x}^{2}+\sigma_{e}^{2} \delta_{e}^{2} & \beta \sigma_{x}^{2} \delta_{x}^{2} \\
\beta \sigma_{x}^{2} \delta_{x}^{2} & \sigma_{x}^{2} \delta_{x}^{2}+\sigma_{u}^{2} \delta_{u}^{2}
\end{array}\right)
$$

Portanto,

$$
\Sigma_{Z}=\left(\begin{array}{cc}
\left(1-\frac{2}{\pi} \delta_{x}^{2}\right) \beta^{2} \sigma_{x}^{2}+\left(1-\frac{2}{\pi} \delta_{e}^{2}\right) \sigma_{e}^{2} & \left(1-\frac{2}{\pi} \delta_{x}^{2}\right) \beta \sigma_{x}^{2} \\
\left(1-\frac{2}{\pi} \delta_{x}^{2}\right) \beta \sigma_{x}^{2} & \left(1-\frac{2}{\pi} \delta_{x}^{2}\right) \sigma_{x}^{2}+\left(1-\frac{2}{\pi} \delta_{u}^{2}\right) \sigma_{u}^{2}
\end{array}\right) .
$$

Casos particulares dos resultados acima são apresentados a seguir.

Corolario 4.6. i. Se $\delta_{x}=\delta_{e}=\delta_{u}=\delta$, então, $\mu_{\delta}=\sqrt{\frac{2}{\pi}} \delta \mathrm{B} \Psi^{\frac{1}{2}}$ e $\Sigma_{\delta}=\frac{2}{\pi} \delta^{2} \Sigma$, de modo que, $\mu_{Z}=\mu+\sqrt{\frac{2}{\pi}} \delta \mathrm{B} \Psi^{\frac{1}{2}}$ e $\Sigma_{Z}=\left(1-\frac{2}{\pi} \delta^{2}\right) \Sigma$.

ii. Se $\delta_{x}=\delta_{e}=\delta_{u}=0$, então $\mu_{\delta}=0$ e $\Sigma_{\delta}=0$, de modo que, $\mu_{Z}=\mu$ e. $\Sigma_{Z}=\Sigma$, que é o resultado para o caso simétrico.

Lema 4.7. Sob as condições do Lema 4.5, quando $n \rightarrow \infty$, temos que,

$$
\overline{\mathbf{Z}} \stackrel{q c}{\rightarrow} \mu_{Z} \quad \text { e } \quad \mathbf{S}_{Z} \stackrel{q c}{\rightarrow} \Sigma_{Z},
$$

Ozán, N. S. 
Demonstração. A prova consiste na aplicação da Lei Forte dos Grandes Números (Sen e Singer, 1993), pois temos $\mathbf{Z}_{1}, \mathbf{Z}_{2}, \ldots, \mathbf{Z}_{n}$, independentes, tal que $E\left(\mathbf{Z}_{i}\right)=\mu_{Z}$ e $\operatorname{Var}\left(\mathrm{Z}_{i}\right)=\Sigma_{Z}=\Sigma-\Sigma_{\delta}$, definida positiva, e de componentes finitos, ver Lema 4.5, então, $\frac{1}{n} \sum_{i=1}^{n} \mathbf{Z}_{i} \stackrel{q c}{\rightarrow} \mu_{Z}$

Para a convergência de $\mathrm{S}_{Z}$, seja,

$\mathbf{S}_{Z}=\frac{1}{n} \sum_{i=1}^{n}\left(\mathbf{Z}_{i}-\overline{\mathbf{Z}}\right)\left(\mathbf{Z}_{i}-\overline{\mathbf{Z}}\right)^{T}=\frac{1}{n} \sum_{i=1}^{n}\left[\left(\mathbf{Z}_{i}-\boldsymbol{\mu}_{Z}\right)-\left(\overline{\mathbf{Z}}-\boldsymbol{\mu}_{Z}\right)\right]\left[\left(\mathbf{Z}_{i}-\boldsymbol{\mu}_{Z}\right)-\left(\overline{\mathbf{Z}}-\boldsymbol{\mu}_{Z}\right)\right]^{T} \mathrm{e}$ como $\overline{\mathbf{Z}} \stackrel{q c}{\rightarrow} \boldsymbol{\mu}$ então temos que, $\mathbf{S}_{Z} \stackrel{q c}{\rightarrow} E\left[\left(\mathbf{Z}_{i}-\boldsymbol{\mu}_{Z}\right)\left(\mathbf{Z}_{i}-\boldsymbol{\mu}_{Z}\right)^{T}\right]=\operatorname{Var}\left(\mathbf{Z}_{i}\right)=\Sigma_{Z}$.

Definindo $\boldsymbol{\epsilon}_{i}=\mathbf{Z}_{i}-E\left(\mathbf{Z}_{i}\right), i=1,2, \ldots n$, pela Proposição 4.4 temos que ,

$$
\epsilon_{i}=\mu+\mathrm{Br}_{i}-\mu-\mu_{\delta}=\mathrm{Br}_{i}-\mu_{\delta}=\mathrm{Br}_{i}-\sqrt{\frac{2}{\pi}} \mathrm{B} \Psi^{\frac{1}{2}} \delta
$$

Portanto, $\epsilon_{i}=\mathrm{B}\left[\mathrm{r}_{i}-\sqrt{\frac{2}{\pi}} \Psi^{\frac{1}{2}} \delta\right]$ ou $\epsilon_{i}=\mathrm{B}\left[\mathrm{r}_{i}-E\left(\mathrm{r}_{i}\right)\right], i=1, \ldots, n$.

Assim podemos considerar $\boldsymbol{\epsilon}_{i}=\mathbf{B r}_{i}^{*}$, com $\mathbf{r}_{i}^{*}=\mathbf{r}_{i}-E\left(\mathbf{r}_{i}\right), i=1,2, \ldots$

Logo, $\mathrm{r}_{i}^{*}=\left(v_{i}^{*}, e_{i}^{*}, u_{i}^{*}\right)^{T}=\left(v_{i}-E\left(v_{i}\right), e_{i}-E\left(e_{i}\right), u_{i}-E\left(u_{i}\right)\right)^{T}$, para todo $i=1,2, \ldots \ldots$

Os momentos de $v_{i}^{*}, e_{i}^{*}$ e $u_{i}^{*}$ são:

$$
\begin{array}{ll}
E\left(v_{i}^{*}\right)=0 & E\left(e_{i}^{*}\right)=0 \\
E\left(v *_{i}^{2}\right)=\sigma_{x}^{2}\left(1-\frac{2}{\pi} \delta_{x}^{2}\right) & E\left(e *_{i}^{2}\right)=\sigma_{e}^{2}\left(1-\frac{2}{\pi} \delta_{e}^{2}\right) \\
E\left(v *_{i}^{3}\right)=\sqrt{\frac{2}{\pi}} \frac{4-\pi}{\pi} \sigma_{x}^{3} \delta_{x}^{3} & E\left(e *_{i}^{3}\right)=\sqrt{\frac{2}{\pi}} \frac{4-\pi}{\pi} \sigma_{e}^{3} \delta_{e}^{3} \\
E\left(v *_{i}^{4}\right)=\sigma_{x}^{4}\left[3-\frac{12}{\pi} \delta_{x}^{2}+\frac{4}{\pi^{2}}(2 \pi-3) \delta_{x}^{4}\right] & E\left(e *_{i}^{4}\right)=\sigma_{e}^{4}\left[3-\frac{12}{\pi} \delta_{e}^{2}+\frac{4}{\pi^{2}}(2 \pi-3) \delta_{e}^{4}\right] \\
E\left(u_{i}^{*}\right)=0 & E\left(u *_{i}^{2}\right)=\sigma_{u}^{2}\left(1-\frac{2}{\pi} \delta_{u}^{2}\right) \\
E\left(u *_{i}^{3}\right)=\sqrt{\frac{2}{\pi}} \frac{4-\pi}{\pi} \sigma_{u}^{3} \delta_{u}^{3} & E\left(u *_{i}^{4}\right)=\sigma_{u}^{4}\left[3-\frac{12}{\pi} \delta_{u}^{2}+\frac{4}{\pi^{2}}(2 \pi-3) \delta_{u}^{4}\right] .
\end{array}
$$

Lema 4.8. Seja $\epsilon_{i}=\mathrm{Br}_{i}^{*}$, com $r_{i}^{*}=\mathbf{r}_{i}-\sqrt{\frac{2}{\pi}} \Psi^{\frac{1}{2}} \delta=\mathbf{r}_{i}-E\left(\mathbf{r}_{i}\right)$, para todo $i=1, \ldots, n$. Se $\Gamma=E\left[\epsilon_{i} \operatorname{vech}^{T}\left(\epsilon_{i} \epsilon_{i}^{T}\right)\right]$, então

$$
\boldsymbol{\Gamma}=\sqrt{\frac{2}{\pi}}\left(\frac{4-\pi}{\pi}\right)\left[\sigma_{x}^{3} \delta_{x}^{3} \mathbf{B}^{*}+\sigma_{e}^{3} \delta_{e}^{3} \mathrm{e}_{12}^{T} \mathbf{e}_{13}+\sigma_{u}^{3} \delta_{u}^{3} \mathbf{e}_{22}^{T} \mathbf{e}_{23}\right]
$$

onde $\mathbf{B}^{*}=\mathbf{b}_{1}\left(\mathbf{b}_{1}^{T} \mathbf{b}_{1}-1, \mathbf{b}_{1}^{T}\right)$, com $\mathbf{b}_{1}=(\beta, 1)^{T}$.

$O$ vetor $\mathrm{e}_{i j}$ é um vetor de dimensão $j$ com o valor um na posição $i$ e zero nas outras componentes, $i \leq j$, isto é, $\mathbf{e}_{i j}=(0,0, \ldots, 1,0)$; por exemplo, $\mathbf{e}_{23}=(0,1,0)$.

Demonstração. Como $\epsilon_{i}=\mathrm{Br}_{i}^{*}$, então,

$$
\epsilon_{i}=\left(\begin{array}{c}
\beta v^{*}+e^{*} \\
v^{*}+u^{*}
\end{array}\right)=\left(\begin{array}{c}
e_{Y} \\
e_{X}
\end{array}\right)
$$

Ozán, N. S. 
de modo que, vech ${ }^{T}\left(\epsilon_{i} \epsilon_{i}^{T}\right)=\left(e_{Y}^{2}, e_{Y} e_{X}, e_{X}^{2}\right)^{T}$ e, portanto,

$$
E\left[\epsilon_{i} \operatorname{vech}^{T}\left(\epsilon_{i} \epsilon_{i}^{T}\right)\right]=\left(\begin{array}{lll}
E\left(e_{Y}^{3}\right) & E\left(e_{Y}^{2} e_{X}\right) & E\left(e_{Y} e_{X}^{2}\right) \\
E\left(e_{Y}^{2} e_{X}\right) & E\left(e_{Y} e_{X}^{2}\right) & E\left(e_{X}^{3}\right)
\end{array}\right) .
$$

Assim, usando as equações (4.25), temos,

$$
\begin{array}{ll}
E\left(e_{Y}^{3}\right)=E\left[\left(\beta v_{i}^{*}+e_{i}^{*}\right)^{3}\right] & =\sqrt{\frac{2}{\pi}} \frac{4-\pi}{\pi}\left(\beta^{3} \sigma_{x}^{3} \delta_{x}^{3}+\sigma_{e}^{3} \delta_{e}^{3}\right) \\
E\left(e_{Y}^{2} e_{X}\right)=E\left[\left(\beta v_{i}^{*}+e_{i}^{*}\right)^{2}\left(v_{i}^{*}+u_{i}^{*}\right)\right] & =\sqrt{\frac{2}{\pi}} \frac{4-\pi}{\pi} \beta^{2} \sigma_{x}^{3} \delta_{x}^{3} \\
E\left(e_{Y} e_{X}^{2}\right)=E\left[\left(\beta v_{i}^{*}+e_{i}^{*}\right)\left(v_{i}^{*}+u_{i}^{*}\right)^{2}\right] & =\sqrt{\frac{2}{\pi}} \frac{4-\pi}{\pi} \beta \sigma_{x}^{3} \delta_{x}^{3} \\
E\left(e_{X}^{3}\right)=E\left[\left(v_{i}^{*}+u_{i}^{*}\right)^{3}\right] & =\sqrt{\frac{2}{\pi}} \frac{4-\pi}{\pi}\left(\sigma_{x}^{3} \delta_{x}^{3}+\sigma_{u}^{3} \delta_{u}^{3}\right) .
\end{array}
$$

Logo,

$$
\begin{aligned}
\Gamma & =\sqrt{\frac{2}{\pi}}\left(\frac{4-\pi}{\pi}\right)\left(\begin{array}{ccc}
\beta^{3} \sigma_{x}^{3} \delta_{x}^{3}+\sigma_{e}^{3} \delta_{e}^{3} & \beta^{2} \sigma_{x}^{3} \delta_{x}^{3} & \beta \sigma_{x}^{3} \delta_{x}^{3} \\
\beta^{2} \sigma_{x}^{3} \delta_{x}^{3} & \beta \sigma_{x}^{3} \delta_{x}^{3} & \sigma_{x}^{3} \delta_{x}^{3}+\sigma_{u}^{3} \delta_{u}^{3}
\end{array}\right)= \\
& =\sqrt{\frac{2}{\pi}\left(\frac{4-\pi}{\pi}\right)}\left[\sigma_{x}^{3} \delta_{x}^{3}\left(\begin{array}{ccc}
\beta^{3} & \beta^{2} & \beta \\
\beta^{2} & \beta & 1
\end{array}\right)+\left(\begin{array}{ccc}
\sigma_{e}^{3} \delta_{e}^{3} & 0 & 0 \\
0 & 0 & \sigma_{u}^{3} \delta_{u}^{3}
\end{array}\right)\right] .
\end{aligned}
$$

Definindo $b_{1}=\left(\begin{array}{c}\beta \\ 1\end{array}\right)$, temos que,

$$
\left(\begin{array}{ccc}
\beta^{3} & \beta^{2} & \beta \\
\beta^{2} & \beta & 1
\end{array}\right)=\left(\begin{array}{c}
\beta \\
1
\end{array}\right)\left(\begin{array}{lll}
\beta^{2} & \beta & 1
\end{array}\right)=\mathbf{b}_{1}\left(\mathbf{b}_{1}^{T} \mathbf{b}_{1}-1, \mathbf{b}_{1}^{T}\right) .
$$

Por outro lado,

$$
\left(\begin{array}{lll}
\sigma_{e}^{3} \delta_{e}^{3} & 0 & 0 \\
0 & 0 & \sigma_{u}^{3} \delta_{u}^{3}
\end{array}\right)=\sigma_{e}^{3} \delta_{e}^{3}\left(\begin{array}{l}
1 \\
0
\end{array}\right)\left(\begin{array}{lll}
1 & 0 & 0
\end{array}\right)+\sigma_{u}^{3} \delta_{u}^{3}\left(\begin{array}{l}
0 \\
1
\end{array}\right)\left(\begin{array}{lll}
0 & 0 & 1
\end{array}\right),
$$

usando a notação $\mathbf{e}_{i j}$, definida acima, para os vetores de dimensão $j$ com o valor um na posição $i$ e zeros nas outras componentes, e $\mathbf{B}^{*}=\mathbf{b}_{1}\left(\mathbf{b}_{1}^{T} \mathbf{b}_{1}-1, \mathbf{b}_{1}^{T}\right)$, obtemos a expressão para $\Gamma$.

Corolario 4.9. $\quad$ i. Se $\delta_{x}=\delta_{e}=\delta_{u}=\delta$, então

$$
\boldsymbol{\Gamma}=\sqrt{\frac{2}{\pi}}\left(\frac{4-\pi}{\pi}\right) \delta^{3}\left[\sigma_{x}^{3} \mathbf{B}^{*}+\sigma_{e}^{3} \mathbf{e}_{12}^{T} \mathbf{e}_{13}+\sigma_{u}^{3} \mathbf{e}_{22}^{T} \mathbf{e}_{23}\right] .
$$

ii. Se $\delta_{x}=\delta_{e}=\delta_{u}=0$ então $\Gamma=0$ e o caso simétrico segue.

Lema 4.10. Seja $\epsilon_{i}=\mathrm{Br}_{i}^{*}, \operatorname{com} r_{i}^{*}=\mathrm{r}_{i}-\sqrt{\frac{2}{\pi}} \Psi^{\frac{1}{2}} \delta=\mathrm{r}_{i}-E\left(\mathrm{r}_{i}\right), i=1, \ldots, n$.

Se $\Lambda=\operatorname{Var}\left[\operatorname{vech}\left(\epsilon_{i} \epsilon_{i}^{T}\right)\right]$, então,

$$
\Lambda=\Lambda_{Z}+\Lambda_{\delta}
$$

Ozán, N. S. 
onde,

$$
\Lambda_{Z}=\left(\begin{array}{ccc}
2 \sigma_{Y Y}^{4} & 2 \sigma_{Y Y}^{2} \sigma_{X Y}^{2} & 2 \sigma_{X X}^{4} \\
2 \sigma_{Y Y}^{2} \sigma_{X Y}^{2} & \sigma_{X Y}^{4}+\sigma_{Y Y}^{2} \sigma_{X X}^{2} & 2 \sigma_{X X}^{2} \sigma_{X Y}^{2} \\
2 \sigma_{X Y}^{4} & 2 \sigma_{X Y}^{2} \sigma_{X X}^{2} & 2 \sigma_{X X}^{4}
\end{array}\right)
$$

com $\sigma_{Y Y}^{2}, \sigma_{X Y}^{2} \sigma_{X X}^{2}$ sendo os elementos da matriz $\Sigma_{Z}$ em (4.28), e

$$
\Lambda_{\delta}=\left(\begin{array}{ccc}
\beta^{4} \sigma_{x}^{4} \delta_{x}^{*}+\sigma_{e}^{4} \delta_{e}^{*} & \beta^{3} \sigma_{x}^{4} \delta_{x}^{*} & \beta^{2} \sigma_{x}^{4} \delta_{x}^{*} \\
\beta^{3} \sigma_{x}^{4} \delta_{x}^{*} & \beta^{2} \sigma_{x}^{4} \delta_{x}^{*} & \beta \sigma_{x}^{4} \delta_{x}^{*} \\
\beta^{2} \sigma_{x}^{4} \delta_{x}^{*} & \beta \sigma_{x}^{4} \delta_{x}^{*} & \sigma_{x}^{4} \delta_{x}^{*}+\sigma_{u}^{4} \delta_{u}^{*}
\end{array}\right)
$$

$\operatorname{com} \delta_{x}^{*}=\frac{8}{\pi}(\pi-3) \delta_{x}^{4}, \delta_{e}^{*}=\frac{8}{\pi}(\pi-3) \delta_{e}^{4}$ e $\delta_{u}^{*}=\frac{8}{\pi}(\pi-3) \delta_{u}^{4}$.

Demonstração. Se $\epsilon_{i}=\mathrm{Br}_{i}^{*}$, então,

$$
\dot{\epsilon}_{i}=\left(\begin{array}{c}
\beta v^{*}+e^{*} \\
v^{*}+u^{*}
\end{array}\right)=\left(\begin{array}{c}
e_{Y} \\
e_{X}
\end{array}\right) \Rightarrow \operatorname{vech}\left(\epsilon_{i} \epsilon_{i}^{T}\right)=\left(\begin{array}{l}
e_{Y}^{2} \\
e_{Y} e_{X} \\
e_{X}^{2}
\end{array}\right),
$$

de modo que $\Lambda=\operatorname{Var}\left[\operatorname{vech}\left(\epsilon_{i} \epsilon_{i}^{T}\right)\right]$, é tal que,

$$
\Lambda=\left(\begin{array}{ccc}
\operatorname{Var}\left(e_{Y}^{2}\right) & \operatorname{cov}\left(e_{Y}^{2}, e_{Y} e_{X}\right) & \operatorname{cov}\left(e_{Y}^{2}, e_{X}^{2}\right) \\
\operatorname{cov}\left(e_{Y}^{2}, e_{Y} e_{X}\right) & \operatorname{Var}\left(e_{Y} e_{X}\right. & \operatorname{cov}\left(e_{Y} e_{X}, e_{X}^{2}\right) \\
\operatorname{cov}\left(e_{Y}^{2}, e_{X}^{2}\right) & \operatorname{cov}\left(e_{Y} e_{Y}, e_{X}^{2}\right) & \operatorname{Var}\left(e_{X}^{2}\right)
\end{array}\right)
$$

Portanto, usando (4.25) temos, após umas série de manipulações algébricas, que

$$
\begin{aligned}
\operatorname{Var}\left(e_{Y}^{2}\right) & =E\left[\left(\beta v^{*}+e^{*}\right)^{4}\right]-E\left[\left(\beta v^{*}+e^{*}\right)^{2}\right]^{2}= \\
& =2 \beta^{4} \sigma_{x}^{4} d_{x}+2 \sigma_{e}^{4} d_{e}+4 \beta^{2} \sigma_{x}^{2} \sigma_{e} d_{x e} \\
\operatorname{cov}\left(e_{Y}^{2}, e_{Y} e_{X}\right) & =E\left[\left(\beta v^{*}+e^{*}\right)^{3}\left(v^{*}+u^{*}\right)\right]-E\left[\left(\beta v^{*}+e^{*}\right)^{2}\right] E\left[\left(\beta v^{*}+e^{*}\right)\left(v^{*}+u^{*}\right)\right]= \\
& =2 \beta^{3} \sigma_{x}^{4} d_{x}+2 \beta \sigma_{x}^{2} \sigma_{e}^{2} d_{x e} \\
\operatorname{cov}\left(e_{Y}^{2}, e_{X}^{2}\right) & =E\left[\left(\beta v^{*}+e^{*}\right)^{2}\left(v^{*}+u^{*}\right)^{2}\right]-E\left[\left(\beta v^{*}+e^{*}\right)^{2}\right] E\left[\left(v^{*}+u^{*}\right)^{2}\right]= \\
& =2 \beta^{2} \sigma_{x}^{4} d_{x} \\
\operatorname{Var}\left(e_{Y} e_{X}\right) & =E\left[\left(\beta v^{*}+e^{*}\right)^{2}\left(v^{*}+u^{*}\right)^{2}\right]-E\left[\left(\beta v^{*}+e^{*}\right)\left(v^{*}+u^{*}\right)\right]^{2}= \\
\operatorname{cov}\left(e_{Y} e_{X}, e_{X}^{2}\right) & =E\left[\left(\beta v^{2}+e^{*}\right)\left(v^{*}+u^{*}\right)^{3}\right]-E\left[\left(\beta v^{*}+e^{*}\right)\left(v^{*}+u^{*}\right)\right] E\left[\left(v^{*}+u^{*}\right)^{2}\right]= \\
& =2 \beta \sigma_{x}^{4} d_{x}+2 \beta \sigma_{x}^{2} \sigma_{u}^{2} d_{x u} \\
\operatorname{Var}\left(e_{X}^{2}\right) & =E\left[\left(v^{*}+u^{*}\right)^{4}\right]-E\left[\left(v^{*}+u^{*}\right)^{2}\right]^{2}= \\
& =2 \sigma_{x}^{4} d_{x}+2 \sigma_{u}^{4} d_{u}+4 \sigma_{x}^{2} \sigma_{u}^{2} d_{x u},
\end{aligned}
$$

onde,

$$
\begin{aligned}
d_{x} & =1-\frac{4}{\pi} \delta_{x}^{2}+\frac{4}{\pi^{2}}(\pi-2) \delta_{x}^{4}=\left(1-\frac{2}{\pi} \delta_{x}^{2}\right)^{2}+\frac{4}{\pi^{2}}(\pi-3) \delta_{x}^{4} \\
d_{e} & =1-\frac{4}{\pi} \delta_{e}^{2}+\frac{4}{\pi^{2}}(\pi-2) \delta_{e}^{4}=\left(1-\frac{2}{\pi} \delta_{e}^{2}\right)^{2}+\frac{4}{\pi^{2}}(\pi-3) \delta_{e}^{4}, \\
d_{u} & =1-\frac{4}{\pi} \delta_{u}^{2}+\frac{4}{\pi^{2}}(\pi-2) \delta_{u}^{4}=\left(1-\frac{2}{\pi} \delta_{u}^{2}\right)^{2}+\frac{4}{\pi^{2}}(\pi-3) \delta_{u}^{4} \\
d_{e u} & =\left(1-\frac{2}{\pi} \delta_{e}^{2}\right)\left(1-\frac{2}{\pi} \delta_{u}^{2}\right), \\
d_{x u} & =\left(1-\frac{2}{\pi} \delta_{x}^{2}\right)\left(1-\frac{2}{\pi} \delta_{u}^{2}\right), \\
d_{x e} & =\left(1-\frac{2}{\pi} \delta_{x}^{2}\right)\left(1-\frac{2}{\pi} \delta_{e}^{2} .\right.
\end{aligned}
$$


Logo, em (4.34), temos,

$$
\Lambda=\left(\begin{array}{ccc}
2 \beta^{4} \sigma_{x}^{4} d_{x}+2 \sigma_{e}^{4} d_{e}+4 \beta^{2} \sigma_{x}^{2} \sigma_{e} d_{x e} & 2 \beta^{3} \sigma_{x}^{4} d_{x}+2 \beta \sigma_{x}^{2} \sigma_{e}^{2} d_{x e} & 2 \beta^{2} \sigma_{x}^{4} d_{x} \\
2 \beta^{3} \sigma_{x}^{4} d_{x}+2 \beta \sigma_{x}^{2} \sigma_{e}^{2} d_{x e} & 2 \beta^{2} \sigma_{x}^{4} d_{x}+\beta^{2} \sigma_{x}^{2} \sigma_{u}^{2} d_{x u}+ & 2 \beta \sigma_{x}^{4} d_{x}+2 \beta \sigma_{x}^{2} \sigma_{u}^{2} d_{x u} \\
& +\sigma_{x}^{2} \sigma_{e}^{2} d_{x e}+\sigma_{e}^{2} \sigma_{u}^{2} d_{e u} & \\
2 \beta^{2} \sigma_{x}^{4} d_{x} & 2 \beta \sigma_{x}^{4} d_{x}+2 \beta \sigma_{x}^{2} \sigma_{u}^{2} d_{x u} & 2 \sigma_{x}^{4} d_{x}+2 \sigma_{u}^{4} d_{u}+4 \sigma_{x}^{2} \sigma_{u}^{2} d_{x u}
\end{array}\right) .
$$

Tendo em conta que os elementos da matriz $\Sigma_{Z}$, são:

$$
\begin{aligned}
& \sigma_{Y Y}^{2}=\left(1-\frac{2}{\pi} \delta_{x}^{2}\right) \beta^{2} \sigma_{x}^{2}+\left(1-\frac{2}{\pi} \delta_{e}^{2}\right) \sigma_{e}^{2} \\
& \sigma_{X X}^{2}=\left(1-\frac{2}{\pi} \delta_{x}^{2}\right) \sigma_{x}^{2}+\left(1-\frac{2}{\pi} \delta_{u}^{2}\right) \sigma_{u}^{2} \\
& \sigma_{X Y}^{2}=\left(1-\frac{2}{\pi} \delta_{x}^{2}\right) \beta \sigma_{x}^{2}
\end{aligned}
$$

com as relações,

$$
\begin{array}{ll}
\sigma_{Y Y}^{2}-\beta \sigma_{X Y}^{2} & =\left(1-\frac{2}{\pi} \delta_{e}^{2}\right) \sigma_{e}^{2} \\
\beta \sigma_{X X}^{2}-\sigma_{X Y}^{2} & =\left(1-\frac{2}{\pi} \delta_{u}^{2}\right) \sigma_{u}^{2} \\
\sigma_{Y Y}^{4} & =\left(1-\frac{2}{\pi} \delta_{x}^{2}\right)^{2} \beta^{4} \sigma_{x}^{4}+\left(1-\frac{2}{\pi} \delta_{e}^{2}\right)^{2} \sigma_{e}^{4}+2 \beta^{2} \sigma_{x}^{2} \sigma_{e}^{2}\left(1-\frac{2}{\pi} \delta_{x}^{2}\right)\left(1-\frac{2}{\pi} \delta_{e}^{2}\right)
\end{array}
$$

temos que os elementos da matriz $\Lambda$; isto é, $\lambda_{i j}$, para $i, j=1,2,3$; são dados por

$$
\begin{array}{rl}
\lambda_{11}= & 2 \beta^{4} \sigma_{x}^{4} d_{x}+2 \sigma_{e}^{4} d_{e}+4 \beta^{2} \sigma_{x}^{2} \sigma_{e} d_{x e} \\
= & \left.2 \beta^{4} \sigma_{x}^{4}\left[\left(1-\frac{2}{\pi} \delta_{x}^{2}\right)^{2}+\frac{4}{\pi^{2}}(\pi-3) \delta_{x}^{4}\right]+2 \sigma_{e}^{4}\left(1-\frac{2}{\pi} \delta_{e}^{2}\right)^{2}+\frac{4}{\pi^{2}}(\pi-3) \delta_{e}^{4}\right]+ \\
& +4 \beta^{2} \sigma_{x}^{2} \sigma_{e}\left(1-\frac{2}{\pi} \delta_{x}^{2}\right)\left(1-\frac{2}{\pi} \delta_{e}^{2}\right) \\
= & 2\left[\left(1-\frac{2}{\pi} \delta_{x}^{2}\right)^{2} \beta^{4} \sigma_{x}^{4}+\left(1-\frac{2}{\pi} \delta_{e}^{2}\right)^{2} \sigma_{e}^{4}\right]+2 \beta^{2} \sigma_{x}^{2} \sigma_{e}^{2}\left(1-\frac{2}{\pi} \delta_{x}^{2}\right)\left(1-\frac{2}{\pi} \delta_{e}^{2}\right)+ \\
& +2 \beta^{4} \sigma_{x}^{4} \frac{4}{\pi^{2}}(\pi-3) \delta_{x}^{4}+2 \sigma_{e}^{4} \frac{4}{\pi^{2}}(\pi-3) \delta_{e}^{4} \\
= & 2 \sigma_{Y Y}^{4}+\frac{8}{\pi^{2}}(\pi-3) \delta_{x}^{4} \beta^{4} \sigma_{x}^{4}+\frac{8}{\pi^{2}}(\pi-3) \delta_{e}^{4} \sigma_{e}^{4} \\
= & 2 \sigma_{Y Y}^{4}+\beta^{4} \sigma_{x}^{4} \delta_{x}^{*}+\sigma_{e}^{4} \delta_{e}^{*} \\
\lambda_{12} & 2 \beta^{3} \sigma_{x}^{4} d_{x}+2 \beta \sigma_{x}^{2} \sigma_{e} d_{x e} \\
= & 2 \beta^{3} \sigma_{x}^{4}\left[\left(1-\frac{2}{\pi} \delta_{x}^{2}\right)^{2}+\frac{4}{\pi^{2}}(\pi-3) \delta_{x}^{4}\right]+2 \beta \sigma_{x}^{2} \sigma_{e}\left(1-\frac{2}{\pi} \delta_{x}^{2}\right)\left(1-\frac{2}{\pi} \delta_{e}^{2}\right) \\
= & 2 \beta\left[\left(1-\frac{2}{\pi} \delta_{x}^{2}\right) \beta \sigma_{x}^{2}\right]^{2}+2 \beta \sigma_{x}^{2} \sigma_{e}^{2}\left(1-\frac{2}{\pi} \delta_{x}^{2}\right)\left(1-\frac{2}{\pi} \delta_{e}^{2}\right)+\beta^{3} \sigma_{x}^{4} \frac{8}{\pi^{2}}(\pi-3) \delta_{x}^{4} \\
= & 2 \beta \sigma_{X Y}^{4}+2 \sigma_{X Y}^{2}\left(\sigma_{Y Y}^{2}-\beta \sigma_{X Y}^{2}\right)+\beta^{3} \sigma_{x}^{4} \frac{8}{\pi^{2}}(\pi-3) \delta_{x}^{4} \\
= & 2 \sigma_{Y Y}^{2} \sigma_{X Y}+\beta^{3} \sigma_{x}^{4} \frac{8}{\pi^{2}}(\pi-3) \delta_{x}^{4} \\
= & 2 \sigma_{Y Y}^{2} \sigma_{X Y}^{2}+\beta^{3} \sigma_{x}^{4} \delta_{x}^{*} \\
= & 2 \beta^{2} \sigma_{x}^{4} d_{x} \\
= & 2 \beta^{2} \sigma_{x}^{4}\left[\left(1-\frac{2}{\pi} \delta_{x}^{2}\right)^{2}+\frac{4}{\pi^{2}}(\pi-3) \delta_{x}^{4}\right] \\
= & 2\left[\left(1-\frac{2}{\pi} \delta_{x}^{2}\right) \beta \sigma_{x}^{2}\right]^{2}+\beta^{2} \sigma_{x}^{4} \frac{8}{\pi^{2}}(\pi-3) \delta_{x}^{4} \\
= & 2 \beta \sigma_{X Y}^{4}+\beta^{2} \sigma_{x}^{4} \frac{8}{\pi^{2}}(\pi-3) \delta_{x}^{4} \\
= & 2 \sigma_{X Y}^{4}+\beta^{2} \sigma_{x}^{4} \delta_{x}^{*}
\end{array}
$$




$$
\begin{array}{rl}
\lambda_{22}= & 2 \beta^{2} \sigma_{x}^{4} d_{x}+\beta^{2} \sigma_{x}^{2} \sigma_{u}^{2} d_{x u}+\sigma_{x}^{2} \sigma_{e}^{2} d_{x e}+\sigma_{e}^{2} \sigma_{u}^{2} d_{e u} \\
= & 2 \beta^{2} \sigma_{x}^{4}\left[\left(1-\frac{2}{\pi} \delta_{x}^{2}\right)^{2}+\frac{4}{\pi^{2}}(\pi-3) \delta_{x}^{4}\right]+\beta \sigma_{x}^{2} \sigma_{u}^{2}\left(1-\frac{2}{\pi} \delta_{x}^{2}\right)\left(1-\frac{2}{\pi} \delta_{u}^{2}\right)+ \\
& +\sigma_{x}^{2} \sigma_{e}^{2}\left(1-\frac{2}{\pi} \delta_{x}^{2}\right)\left(1-\frac{2}{\pi} \delta_{e}^{2}\right)+\sigma_{e}^{2} \sigma_{u}^{2}\left(1-\frac{2}{\pi} \delta_{e}^{2}\right)\left(1-\frac{2}{\pi} \delta_{u}^{2}\right) \\
= & 2 \sigma_{X Y}^{4}+\frac{8}{\pi^{2}}(\pi-3) \delta_{x}^{4} \beta^{2} \sigma_{x}^{4}+\beta \sigma_{x}^{2} \sigma_{u}^{2}\left(1-\frac{2}{\pi} \delta_{x}^{2}\right)\left(1-\frac{2}{\pi} \delta_{u}^{2}\right)+\sigma_{e}^{2}\left(1-\frac{2}{\pi} \delta_{e}^{2}\right) \sigma_{x x}^{2} \\
= & \sigma_{X Y}^{4}+\frac{8}{\pi^{2}}(\pi-3) \delta_{x}^{4} \beta^{2} \sigma_{x}^{4}+\left(\sigma_{Y Y}^{2}-\beta \sigma_{X Y}^{2}\right) \sigma_{X X}^{2}+\sigma_{X Y}^{2}\left(\beta \sigma_{X X}^{2}-\sigma_{X Y}^{2}\right) \\
= & \sigma_{X Y}^{4}+\sigma_{X Y}^{2} \sigma_{X X}^{2}+\frac{8}{\pi^{2}}(\pi-3) \delta_{x}^{4} \beta^{2} \sigma_{x}^{4} \\
= & \sigma_{X Y}^{4}+\sigma_{X Y}^{2} \sigma_{X X}^{2}+\beta^{2} \sigma_{x}^{4} \delta_{x}^{*}, \\
\lambda_{23}, & 2 \beta \sigma_{x}^{4} d_{x}+2 \beta \sigma_{x}^{2} \sigma_{u} d_{x u} \\
= & 2 \sigma_{x}^{4}\left[\left(1-\frac{2}{\pi} \delta_{x}^{2}\right)^{2}+\frac{4}{\pi^{2}}(\pi-3) \delta_{x}^{4}\right]+2 \beta \sigma_{x}^{2} \sigma_{u}\left(1-\frac{2}{\pi} \delta_{x}^{2}\right)\left(1-\frac{2}{\pi} \delta_{u}^{2}\right) \\
= & 2 \frac{1}{\beta} \sigma_{X Y}^{4}+2 \frac{1}{\beta} \sigma_{X Y}^{2}\left(\beta \sigma_{X X}^{2}-\sigma_{X Y}^{2}\right)+\beta \sigma_{x}^{4} \frac{8}{\pi^{2}}(\pi-3) \delta_{x}^{4} \\
= & 2 \sigma_{X Y}^{2} \sigma_{X X}^{2}+\beta \sigma_{x}^{4} \frac{8}{\pi^{2}}(\pi-3) \delta_{x}^{4} \\
= & 2 \sigma_{X Y}^{2} \sigma_{X X}^{2}+\beta \sigma_{x}^{4} \delta_{x}^{*} \\
\lambda_{33} & 2 \sigma_{x}^{4} d_{x}+2 \sigma_{u}^{4} d_{e}+4 \sigma_{x}^{2} \sigma_{u} d_{x u} \\
= & \left.2 \sigma_{x}^{4}\left[\left(1-\frac{2}{\pi} \delta_{x}^{2}\right)^{2}+\frac{4}{\pi^{2}}(\pi-3) \delta_{x}^{4}\right]+2 \sigma_{u}^{4}\left(1-\frac{2}{\pi} \delta_{u}^{2}\right)^{2}+\frac{4}{\pi^{2}}(\pi-3) \delta_{e}^{4}\right]+ \\
& +4 \sigma_{x}^{2} \sigma_{u}\left(1-\frac{2}{\pi} \delta_{x}^{2}\right)\left(1-\frac{2}{\pi} \delta_{u}^{2}\right) \\
= & 2 \sigma_{X X}^{4}+\frac{8}{\pi^{2}}(\pi-3) \delta_{x}^{4} \sigma_{x}^{4}+\frac{8}{\pi^{2}}(\pi-3) \delta_{u}^{4} \sigma_{e}^{4} \\
= & 2 \sigma_{X X}^{4}+\beta^{4} \sigma_{x}^{4} \delta_{x}^{*}+\sigma_{u}^{4} \delta_{u}^{*} . \\
&
\end{array}
$$

Logo os elementos da matriz $\Lambda$ são:

$$
\begin{aligned}
& \lambda_{11}=2 \sigma_{Y Y}^{4}+\beta^{4} \sigma_{x}^{4} \delta_{x}^{*}+\sigma_{e}^{4} \delta_{e}^{*}, \\
& \lambda_{12}=2 \sigma_{Y Y}^{2} \sigma_{X Y}^{2}+\beta^{3} \sigma_{x}^{4} \delta_{x}^{*}, \\
& \lambda_{13}=2 \sigma_{X Y}^{4}+\beta^{2} \sigma_{x}^{4} \delta_{x}^{*}, \\
& \lambda_{22}=\sigma_{X Y}^{4}+\sigma_{X Y}^{2} \sigma_{X X}^{2}+\beta^{2} \sigma_{x}^{4} \delta_{x}^{*}, \\
& \lambda_{23}=\sigma_{X Y}^{2} \sigma_{X X}^{2}+\beta \sigma_{x}^{4} \delta_{x}^{*}, \\
& \lambda_{33}=2 \sigma_{X X}^{4}+\beta^{4} \sigma_{x}^{4} \delta_{x}^{*}+\sigma_{u}^{4} \delta_{u}^{*},
\end{aligned}
$$

$\operatorname{com} \delta_{x}^{*}=\frac{8}{\pi}(\pi-3) \delta_{x}^{4}, \delta_{e}^{*}=\frac{8}{\pi}(\pi-3) \delta_{e}^{4}$ e $\delta_{u}^{*}=\frac{8}{\pi}(\pi-3) \delta_{u}^{4}$.

Portanto, temos,

$$
\Lambda=\left(\begin{array}{ccc}
2 \sigma_{Y Y}^{4}+\beta^{4} \sigma_{x}^{4} \delta_{x}^{*}+\sigma_{e}^{4} \delta_{e}^{*} & 2 \sigma_{Y Y}^{2} \sigma_{X Y}^{2}+\beta^{3} \sigma_{x}^{4} \delta_{x}^{*} & 2 \sigma_{X Y}^{4}+\beta^{2} \sigma_{x}^{4} \delta_{x}^{*} \\
2 \sigma_{Y Y}^{2} \sigma_{X Y}^{2}+\beta^{3} \sigma_{x}^{4} \delta_{x}^{*} & \sigma_{X Y}^{4}+\sigma_{X Y}^{2} \sigma_{X X}^{2}+\beta^{2} \sigma_{x}^{4} \delta_{x}^{*} & \sigma_{X Y}^{2} \sigma_{X X}^{2}+\beta \sigma_{x}^{4} \delta_{x}^{*} \\
2 \sigma_{X Y}^{4}+\beta^{2} \sigma_{x}^{4} \delta_{x}^{*} & \sigma_{X Y}^{2} \sigma_{X X}^{2}+\beta \sigma_{x}^{4} \delta_{x}^{*} & 2 \sigma_{X X}^{4}+\beta^{4} \sigma_{x}^{4} \delta_{x}^{*}+\sigma_{u}^{4}
\end{array}\right)
$$

Agora é simples observar que a matriz acima tem a forma:

$\Lambda=\left(\begin{array}{ccc}2 \sigma_{Y Y}^{4} & 2 \sigma_{Y Y}^{2} \sigma_{X Y}^{2} & 2 \sigma_{X Y}^{4} \\ 2 \sigma_{Y Y}^{2} \sigma_{X Y}^{2} & \sigma_{X Y}^{4}+\sigma_{X Y}^{2} \sigma_{X X}^{2} & \sigma_{X Y}^{2} \sigma_{X X}^{2} \\ 2 \sigma_{X Y}^{4} & \sigma_{X Y}^{2} \sigma_{X X}^{2} & 2 \sigma_{X X}^{4}\end{array}\right)+\left(\begin{array}{ccc}\beta^{4} \sigma_{x}^{4} \delta_{x}^{*}+\sigma_{e}^{4} \delta_{e}^{*} & \beta^{3} \sigma_{x}^{4} \delta_{x}^{*} & \beta^{2} \sigma_{x}^{4} \delta_{x}^{*} \\ \beta^{3} \sigma_{x}^{4} \delta_{x}^{*} & \beta^{2} \sigma_{x}^{4} \delta_{x}^{*} & \beta \sigma_{x}^{4} \delta_{x}^{*} \\ \beta^{2} \sigma_{x}^{4} \delta_{x}^{*} & \beta \sigma_{x}^{4} \delta_{x}^{*} & \beta^{4} \sigma_{x}^{4} \delta_{x}^{*}+\sigma_{u}^{4}\end{array}\right)$.

Logo, $\Lambda$ tem a expressão (4.29), ficando provado o lema. 
Observação 11. Podemos escrever a matriz $\Lambda_{\delta}$ da seguinte forma:

$$
\Lambda_{\delta}=\sigma_{x}^{4} \delta_{x}^{*}\left(\begin{array}{ccc}
\beta^{4} & \beta^{3} & \beta^{2} \\
\beta^{3} & \beta^{2} & \beta \\
\beta^{2} & \beta & 1
\end{array}\right)+\left(\begin{array}{ccc}
\sigma_{e}^{4} \delta_{e}^{*} & 0 & 0 \\
0 & 0 & 0 \\
0 & 0 & \sigma_{u}^{4} \delta_{u}^{*}
\end{array}\right)
$$

e definido a matriz

$$
\mathbf{B}_{1}=\left(\begin{array}{ccc}
\beta^{4} & \beta^{3} & \beta^{2} \\
\beta^{3} & \beta^{2} & \beta \\
\beta^{2} & \beta & 1
\end{array}\right)
$$

a expressão para $\Lambda_{\delta}$ fica,

$$
\Lambda_{\delta}=\sigma_{x}^{4} \delta_{x}^{*} \mathbf{B}_{1}+\sigma_{e}^{4} \delta_{e}^{*} e_{12}^{T} e_{13}+\sigma_{u}^{4} \delta_{u}^{*} e_{22}^{T} e_{33} .
$$

Corolario 4.11. i. Se $\delta_{x}=\delta_{e}=\delta_{u}=\delta$, então, $\Lambda_{Z}=\left(1-\frac{1}{\pi} \delta^{2}\right) \Sigma e$

$$
\Lambda_{\delta}=\delta\left[\sigma_{x}^{4} \mathbf{B}_{1}+\sigma_{e}^{4} \mathbf{e}_{12}^{T} \mathbf{e}_{13}+\sigma_{u}^{4} \mathbf{e}_{22}^{T} \mathbf{e}_{33}\right]
$$

de modo que

$$
\Lambda=\left(1-\frac{1}{\pi} \delta^{2}\right) \Sigma+\Lambda_{\delta}
$$

ii. Seja $\delta_{x}=\delta_{e}=\delta_{u}=\delta=0$. Assim $\Lambda_{\delta}=0$.

Neste caso os componentes $\Sigma_{Z}$ são,

$$
\begin{aligned}
& \sigma_{Y Y}^{2}=\left(1-\frac{2}{\pi} \delta_{x}^{2}\right) \beta^{2} \sigma_{x}^{2}+\left(1-\frac{2}{\pi} \delta_{e}^{2}\right) \sigma_{e}^{2} \quad \sigma_{Y Y}^{2}=\beta^{2} \sigma_{x}^{2}+\sigma_{e}^{2} \\
& \sigma_{Y X}^{2}=\left(1-\frac{2}{\pi} \delta_{x}^{2}\right) \beta \sigma_{x}^{2} \quad \Rightarrow \sigma_{Y X}^{2}=\beta \sigma_{x}^{2} \\
& \sigma_{X X}^{2}=\left(1-\frac{2}{\pi} \delta_{x}^{2}\right) \sigma_{x}^{2}+\left(1-\frac{2}{\pi} \delta_{u}^{2}\right) \sigma_{u}^{2} \quad \sigma_{X X}^{2}=\sigma_{x}^{2}+\sigma_{u}^{2},
\end{aligned}
$$

temos que,

$$
\Lambda=\Lambda_{Z}=\left(\begin{array}{ccc}
2\left(\beta^{2} \sigma_{x}^{2}+\sigma_{e}^{2}\right)^{4} & 2\left(\beta^{2} \sigma_{x}^{2}+\sigma_{e}^{2}\right)^{2}\left(\sigma_{x}^{2}+\sigma_{u}^{2}\right)^{2} & 2 \beta^{2} \sigma_{x}^{4} \\
2\left(\beta^{2} \sigma_{x}^{2}+\sigma_{e}^{2}\right)^{2}\left(\sigma_{x}^{2}+\sigma_{u}^{2}\right)^{2} & 2\left(\beta \sigma_{x}^{2}\right)^{4}+\left(\beta \sigma_{x}^{2}\right)^{2}\left(\sigma_{x}^{2}+\sigma_{u}^{2}\right)^{2} & \beta^{2} \sigma_{x}^{4}\left(\sigma_{x}^{2}+\sigma_{u}^{2}\right)^{2} \\
2 \beta^{2} \sigma_{x}^{4} & \beta^{2} \sigma_{x}^{4}\left(\sigma_{x}^{2}+\sigma_{u}^{2}\right)^{2} & 2\left(\sigma_{x}^{2}+\sigma_{u}^{2}\right)^{2} .
\end{array}\right)
$$

Este resultado coincide com os resultados obtidos para o modelo normal simétrico em Arellano-Valle, Bolfarine, Vilca-Labra (1996).

Como conseqüência dos resultados acima temos que:

1. $\epsilon_{i} \sim \operatorname{SNI}\left(0, \Sigma_{Z}\right), i=1,2, \ldots$

2. $\mathrm{r}_{i}$ tem momento de quarta ordem finito; portanto, $\Lambda=\operatorname{Var}\left[\operatorname{vech}\left(\epsilon_{1} \epsilon_{1}^{\prime}\right)\right]<\infty$. 
3. Quando $n \rightarrow \infty$,

$$
\overline{\mathrm{Z}} \stackrel{q c}{\rightarrow} \mu_{Z} \quad e \quad \mathrm{~S}_{Z} \stackrel{q c}{\rightarrow} \Sigma_{Z}
$$

Assim, as suposições $A_{1}, A_{2}$ e $A_{3}$ do Lema 2.1 e as condições do Teorema 2.1 em ArellanoValle, Bolfarine e Gasco (2001) estão verificadas e podemos enunciar o seguinte teorema.

Teorema 4.12. Sejam $\mathbf{Z}_{1}, \mathbf{Z}_{2}, \ldots, \mathbf{Z}_{n}$, independentes tal que $\mathbf{Z}_{i}=\boldsymbol{\mu}+\mathbf{B r}_{i}$ com $\mathbf{r}_{i} \stackrel{i i d}{\sim} S N I_{3}(0, \Psi, \lambda), i=1, \ldots, n, \operatorname{com} \Psi=\operatorname{diag}\left\{\sigma_{x}^{2}, \sigma_{e}^{2}, \sigma_{u}^{2}\right\}$ e $\boldsymbol{\lambda}=\left(\lambda_{x}, \lambda_{e}, \lambda_{u}\right)^{T}$, sendo $\mathbf{r}_{i}=\left(v_{i}, e_{i}, u_{i}\right)^{T}, v_{i}=x_{i}-\mu_{x}$, tal que $v_{i}, e_{i}$ e $u_{i}$ são independentes. Temos então que,

$$
\left(\begin{array}{c}
\sqrt{n}\left(\overline{\mathbf{Z}}-\boldsymbol{\mu}_{Z}\right) \\
\sqrt{n} \operatorname{vech}\left(\mathbf{S}_{Z}-\mathbf{\Sigma}_{Z}\right)
\end{array}\right) \stackrel{d}{\rightarrow} N_{5}\left(\left(\begin{array}{l}
0 \\
0
\end{array}\right) ;\left(\begin{array}{cc}
\boldsymbol{\Sigma}_{Z} & \boldsymbol{\Gamma} \\
\boldsymbol{\Gamma}^{T} & \Lambda
\end{array}\right)\right),
$$

com,

- $\boldsymbol{\Sigma}_{Z}=\boldsymbol{\Sigma}-\boldsymbol{\Sigma}_{\delta} \operatorname{com} \boldsymbol{\Sigma}=\mathrm{B} \Psi \mathrm{B}^{T}$ e $\boldsymbol{\Sigma}_{\delta}=\frac{2}{\pi} \mathrm{BD}(\boldsymbol{\delta}) \Psi \mathrm{D}(\delta) \mathbf{B}^{T}$, tal que, $\Psi=\operatorname{diag}\left\{\sigma_{x}^{2}, \sigma_{e}^{2}, \sigma_{u}^{2}\right\}$ $e \mathbf{D}(\boldsymbol{\delta})=\operatorname{diag}\left\{\delta_{x}, \delta_{e}, \delta_{u}\right\}$;

- $\boldsymbol{\Gamma}=\sqrt{\frac{2}{\pi}} \frac{4-\pi}{\pi}\left[\sigma_{x}^{3} \delta_{x}^{3} \mathbf{B}^{*}+\sigma_{e}^{3} \delta_{e}^{3} e_{12}^{T} e_{13}+\sigma_{u}^{3} \delta_{u}^{3} e_{22}^{T} e_{23}\right]$, onde $\mathbf{B}^{*}=\mathbf{b}\left(\mathbf{b}^{T} b-1, \mathbf{b}^{T}\right)$, com $\mathbf{b}=(\beta, 1)^{T}$. O vetor $\mathbf{e}_{i j}$ é um vetor de dimensão $j$ com o valor um na posição $i$ e zero nas outras componentes, $i \leq j$;

- $\Lambda=\Lambda_{Z}+\Lambda_{\delta}$, com os respectivos valores de $\Lambda_{Z}$ e $\Lambda_{\delta}$ determinados em (4.31).

Demonstração. Consideremos $\epsilon_{1}, \epsilon_{2}, \ldots, \epsilon_{n}$, tal que $\epsilon_{i}=\mathbf{Z}_{i}-E\left(\mathbf{Z}_{i}\right)$, então, pelo Teorema 2.1 em Arellano-Valle, Bolfarine e Gasco (2001) temos que a distribuição conjunta de $\left(\bar{Z}, S_{Z}\right)$ é Normal de dimensão cinco, ou seja,

$$
\left(\begin{array}{c}
\sqrt{n}(\overline{\mathbf{Z}}-\mu) \\
\sqrt{n} \operatorname{vech}\left(\mathbf{S}_{Z}-\mathbf{\Sigma}_{Z}\right)
\end{array}\right) \stackrel{d}{\rightarrow} N_{5}(0, \Omega)
$$

com $\Omega$ obtido como:

- $\operatorname{Var}\left[\sqrt{n}\left(\overline{\mathbf{Z}}-\boldsymbol{\mu}_{Z}\right)\right]=n \operatorname{Var}(\overline{\mathbf{Z}})=\boldsymbol{\Sigma}_{Z}$, onde $\boldsymbol{\Sigma}_{Z}$ é dada no Lema 4.5.

- $\operatorname{Var}\left[\sqrt{n} \operatorname{vech}\left(\mathbf{S}_{Z}-\mathbf{\Sigma}_{Z}\right)\right]=n \operatorname{Var}\left[\operatorname{vech}\left(\mathbf{S}_{Z}\right)\right]=$ $=n \operatorname{Var}\left[\operatorname{vech}\left(\frac{1}{n} \sum_{i=1}^{n}\left(\mathrm{Z}_{i}-\mu_{Z}\right)\left(\mathrm{Z}_{i}-\mu_{Z}\right)^{T}\right)\right]=n \operatorname{Var}\left[\operatorname{vech}\left(\frac{1}{n} \sum_{i=1}^{n} \epsilon_{i} \epsilon_{i}^{T}\right)\right]=$ $=\operatorname{Var}\left[\operatorname{vech}\left(\epsilon_{i} \epsilon_{i}^{T}\right)\right]=\Lambda$, onde $\Lambda$ é dada no Lema 4.10.

- $\operatorname{cov}\left[\sqrt{n}\left(\overline{\mathbf{Z}}-\boldsymbol{\mu}_{Z}\right), \sqrt{n} \operatorname{vech}^{T}\left(\mathrm{~S}_{Z}-\Sigma_{Z}\right)\right]=$ $=n \operatorname{cov}\left[\frac{1}{n} \sum_{i=1}^{n}\left(\mathbf{Z}_{i}-\boldsymbol{\mu}_{Z}\right), \operatorname{vech}^{T}\left(\frac{1}{n} \sum_{i=1}^{n}\left(\left(\mathbf{Z}_{i}-\boldsymbol{\mu}_{Z}\right)\left(\mathbf{Z}_{i}-\boldsymbol{\mu}_{Z}\right)^{T}-\mathbf{\Sigma}_{Z}\right)\right)\right]=$ 


$$
\begin{aligned}
& =\frac{1}{n} \operatorname{cov}\left[\sum_{i=1}^{n} \epsilon_{i}, \operatorname{vech}^{T}\left(\sum_{i=1}^{n}\left(\epsilon_{i} \epsilon_{i}^{T}-\Sigma_{Z}\right)\right)\right]= \\
& =\frac{1}{n} E\left[\sum_{i=1}^{n} \epsilon_{i} \operatorname{vech}^{T}\left(\sum_{i=1}^{n}\left(\epsilon_{i} \epsilon_{i}^{T}-\Sigma_{Z}\right)\right)\right]= \\
& =E\left[\epsilon_{i} \operatorname{vech}^{T}\left(\epsilon_{i} \epsilon_{i}^{T}\right)\right]=\Gamma, \text { onde a matriz } \boldsymbol{\Gamma} \text { é dada no Lema 4.8. }
\end{aligned}
$$

Assim, $\Omega$ é dada por:

$$
\Omega=\left(\begin{array}{ll}
\Sigma_{Z} & \Gamma \\
\Gamma^{T} & \Lambda
\end{array}\right)
$$

e portanto o teorema esta provado.

Os resultados assintóticos obtido para $\left(\overline{\mathbf{Z}}, \mathbf{S}_{Z}\right)$ são importantes no estudo da distribuição assintótica dos estimadores de $\beta$. A inferência no modelo normal assimétrico com erro nas variáveis é desenvolvido no proximo capítulo, para alguns casos especiais. 
Capítulo 5

\section{Inferência no Modelo Normal Assimétrico com Erros nas Variáveis}

\subsection{Introdução}

Neste capítulo abordamos a inferência no modelo normal assimétrico com erros nas variáveis e em especial a estimação do parâmetro $\beta$.

Primeiramente, apresentamos o problema da estimação dos parâmetros em forma geral, assumindo condições para evitar a não identificabilidade do modelo. Analisamos a consistência do estimador do parâmetro $\beta$ e derivamos a variância assintótica do mesmo.

A seguir, discutimos a estimação por máxima verossimilhança. Nos deparamos com a dificuldade de não haver expressões fechadas para o sistema de equações das derivadas do logaritmo da função de verossimilhança. Portanto, maximizamos o logaritmo da função de verossimilhança usando um método iterativo utilizando a linguagem de programação $O x$. Realizamos um estudo de simulação para analisar o comportamento dos estimadores.

Finalmente, apresentamos uma aplicação a um conjunto de dados reais, fornecidos pelo Sistema Único de Saúde (SUS) do Ministério de Saúde do Brasil.

\subsection{Estimação}

Seja $\mathbf{Z}_{1}, \ldots, \mathbf{Z}_{n}$ uma amostra aleatória de $n$ observações independentes, tal que,

$$
\mathrm{Z}_{i}=\boldsymbol{\mu}+\mathrm{Br}_{i}, \quad \text { com } i=1, \ldots, n
$$

onde,

$$
\mathbf{Z}_{i}=\left(\begin{array}{c}
Y_{i} \\
X_{i}
\end{array}\right), \quad \boldsymbol{\mu}=\left(\begin{array}{c}
\alpha+\beta \mu_{x} \\
\mu_{x}
\end{array}\right), \quad \mathbf{r}_{i}=\left(\begin{array}{c}
v_{i} \\
e_{i} \\
u_{i}
\end{array}\right)=\left(\begin{array}{c}
x_{i}-\mu_{x} \\
e_{i} \\
u_{i}
\end{array}\right), \quad \mathbf{B}=\left(\begin{array}{ccc}
\beta & 1 & 0 \\
1 & 0 & 1
\end{array}\right),
$$

com $\mathbf{r}_{i}=\left(v_{i}, e_{i}, u_{i}\right)^{T} \stackrel{\text { ind }}{\sim} S N I_{3}(0, \Psi, \boldsymbol{\lambda})$, onde $\Psi=\operatorname{diag}\left\{\sigma_{x}^{2}, \sigma_{e}^{2}, \sigma_{u}^{2}\right\}$ e $\boldsymbol{\lambda}=\left(\lambda_{x}, \lambda_{e}, \lambda_{u}\right)^{T}$. 
Considerando o vetor de parâmetros $\theta^{*}=\left(\theta^{T}, \lambda^{T}\right)=\left(\alpha, \beta, \mu_{x}, \sigma_{x}^{2}, \sigma_{e}^{2}, \sigma_{u}^{2}, \lambda_{x}, \lambda_{e}, \lambda_{u}\right)^{T}$, é de interesse estimar principalmente o parâmetro $\beta$, tendo que fazer suposições sobre as variâncias para evitar a não identificabilidade do modelo.

A primeira proposta de estimador para $\beta$ é considerar o estimador usual no modelo normal desenvolvido na literatura (Fuller (1987), Bolfarine e Cordani (1993)).

\subsubsection{Variância $\sigma_{u}^{* 2}$ conhecida}

O estimador para o parâmetro $\beta$ no caso do modelo normal (Fuller, 1987), com $\sigma_{u}^{2}$ conhecida é $\widehat{\beta}=\frac{S_{Y X}}{S_{X X}-\sigma_{u}^{2}}$, onde $S_{Y X}$ e $S_{X X}$ são as componentes da matriz de covariância amostral $\mathbf{S}_{Z}$. Pelos resultados assintóticos obtidos no Capítulo 4 , temos que, $\mathbf{S}_{Z} \stackrel{q c}{\rightarrow} \Sigma_{Z}$, onde,

$$
\Sigma_{Z}=\left(\begin{array}{cc}
\left(1-\frac{2}{\pi} \delta_{x}^{2}\right) \beta^{2} \sigma_{x}^{2}+\left(1-\frac{2}{\pi} \delta_{e}^{2}\right) \sigma_{e}^{2} & \left(1-\frac{2}{\pi} \delta_{x}^{2}\right) \beta \sigma_{x}^{2} \\
\left(1-\frac{2}{\pi} \delta_{x}^{2}\right) \beta \sigma_{x}^{2} & \left(1-\frac{2}{\pi} \delta_{x}^{2}\right) \sigma_{x}^{2}+\left(1-\frac{2}{\pi} \delta_{u}^{2}\right) \sigma_{u}^{2}
\end{array}\right) .
$$

Definindo $\widehat{\beta}_{1}=\frac{S_{Y X}}{S_{X X}-\sigma_{u}^{* 2}}$, temos que,

$$
\widehat{\beta}_{1}=\frac{S_{Y X}}{S_{X X}-\sigma_{u}^{* 2}} \stackrel{q c}{\rightarrow} \frac{\beta \sigma_{x}^{2}\left(1-\frac{2}{\pi} \delta_{x}^{2}\right)}{\sigma_{x}^{2}\left(1-\frac{2}{\pi} \delta_{x}^{2}\right)+\sigma_{u}^{2}\left(1-\frac{2}{\pi} \delta_{u}^{2}\right)-\sigma_{u}^{* 2}},
$$

de modo que se $\sigma_{u}^{* 2}=\sigma_{u}^{2}\left(1-\frac{2}{\pi} \delta_{u}^{2}\right)$, então, $\widehat{\beta}_{1}$ é consistente.

Logo, consideramos o estimador,

$$
\widehat{\beta}_{1}=\frac{S_{Y X}}{S_{X X}-\sigma_{u}^{* 2}} \quad \text { com } \quad \sigma_{u}^{* 2}=\sigma_{u}^{2}\left(1-\frac{2}{\pi} \delta_{u}^{2}\right) \quad \text { conhecida } .
$$

A condição em (5.3.) implica que temos que conhecer a variância $\sigma_{u}^{2}$, analogamente ao requerido no modelo normal usual, e também o parâmetro de assimetria $\delta_{u}$.

Destacamos que se estudamos o caso particular onde somente a covariável não observada, $x$, tem distribuição assimétrica, ou seja o parâmetro $\delta_{u}$ é conhecido e igual a zero. Assim o estimador $\widehat{\beta}_{1}$ coincide com o estimador usual do modelo normal e é importante ressaltar a inluência da assimetria na variância assintótica.

Derivamos, a seguir, a distribuição assintótica de $\widehat{\beta}_{1}$.

Proposição 5.1. Seja $\mathbf{Z}_{i} \operatorname{com}$ em (5.1), $i=1, \ldots, n$, e $\widehat{\beta}_{1}=\frac{S_{Y X}}{S_{X X}-\sigma_{u}^{* 2}}$ o estimador do parâmetro $\beta$, tal que $\sigma_{u}^{* 2}=\sigma_{u}^{2}\left(1-\frac{2}{\pi} \delta_{u}^{2}\right)$ é conhecida. Então,

$$
\sqrt{n}\left(\widehat{\beta}_{1}-\beta\right) \stackrel{d}{\rightarrow} N\left(0, \Omega_{1}\right)
$$

onde,

$$
\Omega_{1}=\frac{1}{\left(1-\frac{2}{\pi} \delta_{x}^{2}\right)^{2} \sigma_{x}^{4}}\left(\lambda_{22}-2 \beta \sigma_{x}^{2} \lambda_{23}+\beta^{2} \sigma_{x}^{4} \lambda_{33}\right)
$$

Ozán, N. S. 
tal que,

$$
\begin{aligned}
& \lambda_{22}=\sigma_{X Y}^{4}+\sigma_{X Y}^{2} \sigma_{X X}^{2}+\beta^{2} \sigma_{x}^{4} \delta_{x}^{*} \\
& \lambda_{23}=\sigma_{X Y}^{2} \sigma_{X X}^{2}+\beta \sigma_{x}^{4} \delta_{x}^{*} \\
& \lambda_{33}=2 \sigma_{X X}^{4}+\beta^{4} \sigma_{x}^{4} \delta_{x}^{*}+\sigma_{u}^{4} \delta_{u}^{*}
\end{aligned}
$$

com,

$$
\begin{array}{ll}
\sigma_{Y Y}^{2}=\left(1-\frac{2}{\pi} \delta_{x}^{2}\right) \beta^{2} \sigma_{x}^{2}+\left(1-\frac{2}{\pi} \delta_{e}^{2}\right) \sigma_{e}^{2} & \delta_{x}^{*}=\frac{8}{\pi}(\pi-3) \delta_{x}^{4} \\
\sigma_{X X}^{2}=\left(1-\frac{2}{\pi} \delta_{x}^{2}\right) \sigma_{x}^{2}+\left(1-\frac{2}{\pi} \delta_{u}^{2}\right) \sigma_{u}^{2} & \delta_{e}^{*}=\frac{8}{\pi}(\pi-3) \delta_{e}^{4} \\
\sigma_{X Y}^{2}=\left(1-\frac{2}{\pi} \delta_{x}^{2}\right) \beta \sigma_{x}^{2} & \delta_{u}^{*}=\frac{8}{\pi}(\pi-3) \delta_{u}^{4} .
\end{array}
$$

Demonstração. Usando o Teorema 4.2. e aplicando o método delta (Sen e Singer, 1993), temos que,

$$
\sqrt{n}\left(f\left[\operatorname{vech}\left(\mathbf{S}_{Z}\right)\right]-f\left[\operatorname{vech}\left(\Sigma_{Z}\right)\right]\right) \stackrel{d}{\rightarrow} N\left(0, \Omega_{1}\right),
$$

sendo $f$ uma função continua e com derivada no espaço paramétrico, e $\Omega_{1}=\Pi \Lambda \Pi^{T}$, onde, $\Lambda$ é a matriz de covariâncias do Lema 4.4 , e $\Pi=\frac{\partial f}{\partial\left(v^{2} h^{T} \Sigma_{Z}\right)}$, com $\Sigma_{Z}$ segundo (5.2.).

Definindo $f\left((x, y, z)^{T}\right)=\frac{y}{z-\sigma_{u}^{*}}$, temos que $f\left[\operatorname{vech}\left(\mathbf{S}_{Z}\right)\right]=\widehat{\beta}_{1}$ e $f\left[\operatorname{vech}\left(\boldsymbol{\Sigma}_{Z}\right)\right]=\beta$.

Por outro lado, $\frac{\partial f}{\partial(x, y, z)}=\frac{1}{z-\sigma_{u}^{*}}\left(0,1,-\frac{y}{z-\sigma_{u}^{*}}\right)$ e, portanto,

$$
\Pi=\frac{\partial f}{\partial\left(\operatorname{vech}^{T} \Sigma_{Z}\right)}=\frac{1}{\sigma_{X X}^{2}-\sigma_{u}^{*}}\left(0,1,-\frac{\sigma_{Y X}^{2}}{\sigma_{X X}^{2}-\sigma_{u}^{*}}\right) .
$$

Sendo $\Lambda$ a matriz de covariâncias do Lema 4.4, isto é,

$$
\Lambda=\left(\begin{array}{ccc}
\lambda_{11} & \lambda_{12} & \lambda_{13} \\
\ldots & \lambda_{22} & \lambda_{23} \\
\ldots & \cdots & \lambda_{33}
\end{array}\right)
$$

onde,

$$
\begin{aligned}
& \lambda_{11}=2 \sigma_{Y Y}^{4}+\beta^{4} \sigma_{x}^{4} \delta_{x}^{*}+\sigma_{e}^{4} \delta_{e}^{*}, \\
& \lambda_{12}=2 \sigma_{Y Y}^{2} \sigma_{X Y}^{2}+\beta^{3} \sigma_{x}^{4} \delta_{x}^{*}, \\
& \lambda_{13}=2 \sigma_{X Y}^{4}+\beta^{2} \sigma_{x}^{4} \delta_{x}^{*}, \\
& \lambda_{22}=\sigma_{X Y}^{4}+\sigma_{X Y}^{2} \sigma_{X X}^{2}+\beta^{2} \sigma_{x}^{4} \delta_{x}^{*}, \\
& \lambda_{23}=\sigma_{X Y}^{2} \sigma_{X X}^{2}+\beta \sigma_{x}^{4} \delta_{x}^{*} \\
& \lambda_{33}=2 \sigma_{X X}^{4}+\beta^{4} \sigma_{x}^{4} \delta_{x}^{*}+\sigma_{u}^{4} \delta_{u}^{*},
\end{aligned}
$$

$\operatorname{com} \delta_{x}^{*}=\frac{8}{\pi}(\pi-3) \delta_{x}^{4}, \delta_{e}^{*}=\frac{8}{\pi}(\pi-3) \delta_{e}^{4}$ e $\delta_{u}^{*}=\frac{8}{\pi}(\pi-3) \delta_{u}^{4}$.

Logo,

$$
\begin{aligned}
\Omega_{1} & =\frac{1}{\sigma_{X X}^{2}-\sigma_{u}^{*}}\left(0,1,-\frac{\sigma_{Y X}^{2}}{\sigma_{X X}^{2}-\sigma_{u}^{*}}\right)\left(\begin{array}{ccc}
\lambda_{11} & \lambda_{12} & \lambda_{13} \\
\ldots & \lambda_{22} & \lambda_{23} \\
\ldots & \ldots & \lambda_{33}
\end{array}\right) \frac{1}{\sigma_{X X}^{2}-\sigma_{u}^{*}}\left(\begin{array}{c}
0 \\
1 \\
-\frac{\sigma_{Y X}^{2}}{\sigma_{X X}^{2}-\sigma_{u}^{*}}
\end{array}\right) \\
& =\frac{1}{\left(\sigma_{X X}^{2}-\sigma_{u}^{*}\right)^{2}}\left(\lambda_{22}-2 \beta \sigma_{x}^{2} \lambda_{23}+\beta^{2} \sigma_{x}^{4} \lambda_{33}\right),
\end{aligned}
$$

Ozán, N. S. 
onde,

$$
\sigma_{X X}^{2}-\sigma_{u}^{* 2}=\left(1-\frac{2}{\pi} \delta_{x}^{2}\right) \sigma_{x}^{2}+\left(1-\frac{2}{\pi} \delta_{u}^{2}\right) \sigma_{u}^{2}-\left(1-\frac{2}{\pi} \delta_{u}^{2}\right) \sigma_{u}^{2}=\left(1-\frac{2}{\pi} \delta_{x}^{2}\right) \sigma_{x}^{2},
$$

e $\lambda_{22}, \lambda_{23}$ e $\lambda_{33}$ definidos segundo as expressões em (5.5). Deste modo fica provada a proposição.

Analisamos agora o caso particular do modelo onde assumimos assimetria só na covariável não observada $x$. Consideramos $\mathbf{Z}_{i}$ como em (5.1), $i=1, \ldots, n$, e assumimos que $\mathbf{r}_{i}=\left(v_{i}, e_{i}, u_{i}\right)^{T} \stackrel{i i d}{\sim} S N I_{3}(\mathbf{0}, \Psi, \boldsymbol{\lambda})$, onde $\boldsymbol{\Psi}=\operatorname{diag}\left\{\sigma_{x}^{2}, \sigma_{e}^{2}, \sigma_{u}^{2}\right\}$ e $\boldsymbol{\lambda}=\left(\lambda_{x}, 0,0\right)^{T}$. Portanto, $\sigma_{u}^{* 2}=\sigma_{u}^{2}\left(1-\frac{2}{\pi} \delta_{u}^{2}\right)=\sigma_{u}^{2}$ e $\widehat{\beta}_{1}^{*}=\frac{S_{Y X}}{S_{X X-\sigma_{u}^{2}}}$.

Derivamos, a seguir, a variância assintótica para o estimador de $\beta$ neste caso.

Corolario 5.2. Seja $\mathbf{Z}_{i}$ com em (5.1), $i=1, \ldots, n$ e consideramos $\lambda_{e}=\lambda_{u}=0$, ou seja, $\delta_{e}=\delta_{u}=0$. Então, $\widehat{\beta}_{1}^{*}=\frac{S_{Y X}}{S_{X X}-\sigma_{u}^{* 2}}$, com $\sigma_{u}^{2}$ conhecida, $e$

$$
\sqrt{n}\left(\widehat{\beta_{1}^{*}}-\beta\right) \stackrel{d}{\rightarrow} N\left(0, \Omega_{1}^{*}\right),
$$

onde,

tal que,

$$
\Omega_{1}^{*}=\frac{1}{\left(1-\frac{2}{\pi} \delta_{x}^{2}\right)^{2} \sigma_{x}^{4}}\left(\lambda_{22}^{*}-2 \beta \sigma_{x}^{2} \lambda_{23}^{*}+\beta^{2} \sigma_{x}^{4} \lambda_{33}^{*}\right)
$$

$$
\lambda_{22}^{*}=\sigma_{X Y}^{* 4}+\sigma_{X Y}^{* 2} \sigma_{X X}^{* 2}+\beta^{2} \sigma_{x}^{4} \delta_{x}^{*}, \quad \lambda_{23}^{*}=\sigma_{X Y}^{* 2} \sigma_{X X}^{* 2}+\beta \sigma_{x}^{4} \delta_{x}^{*}, \quad \lambda_{33}^{*}=2 \sigma_{X X}^{* 4}+\beta^{4} \sigma_{x}^{4} \delta_{x}^{*},
$$

com,

$$
\sigma_{Y Y}^{* 2}=\left(1-\frac{2}{\pi} \delta_{x}^{2}\right) \beta^{2} \sigma_{x}^{2}+\sigma_{e}^{2}, \quad \sigma_{X X}^{* 2}=\left(1-\frac{2}{\pi} \delta_{x}^{2}\right) \sigma_{x}^{2}+\sigma_{u}^{2}, \quad \sigma_{X Y}^{2 *}=\left(1-\frac{2}{\pi} \delta_{x}^{2}\right) \beta \sigma_{x}^{2},
$$

$e \delta_{x}^{*}=\frac{8}{\pi}(\pi-3) \delta_{x}^{4}$.

Demonstração. Considerando $\Sigma_{Z}$ em (5.2) com a hipótese $\delta_{e}=\delta_{u}=0$, temos que,

$$
\sigma_{Y Y}^{* 2}=\left(1-\frac{2}{\pi} \delta_{x}^{2}\right) \beta^{2} \sigma_{x}^{2}+\sigma_{e}^{2}, \quad \sigma_{X X}^{* 2}=\left(1-\frac{2}{\pi} \delta_{x}^{2}\right) \sigma_{x}^{2}+\sigma_{u}^{2}, \quad \sigma_{X Y}^{2 *}=\left(1-\frac{2}{\pi} \delta_{x}^{2}\right) \beta \sigma_{x}^{2} .
$$

Utilizando as expressões (5.4.) para $\lambda_{22}, \lambda_{23}$ e $\lambda_{33}$, calculamos ditos valores sob a hipótese do corolário, obtendo,

$$
\lambda_{22}^{*}=\sigma_{X Y}^{* 4}+\sigma_{X Y}^{* 2} \sigma_{X X}^{* 2}+\beta^{2} \sigma_{x}^{4} \delta_{x}^{*}, \quad \lambda_{23}^{*}=\sigma_{X Y}^{* 2} \sigma_{X X}^{* 2}+\not \beta \sigma_{x}^{4} \delta_{x}^{*}, \quad \lambda_{33}^{*}=2 \sigma_{X X}^{* 4}+\beta^{4} \sigma_{x}^{4} \delta_{x}^{*}
$$

Portanto, usando o resultado da proposição provada acima, temos que a variância assintótica $\operatorname{para} \widehat{\beta}_{1}^{*}$,

$$
\Omega_{1}^{*}=\frac{1}{\left(1-\frac{2}{\pi} \delta_{x}^{2}\right)^{2} \sigma_{x}^{4}}\left(\lambda_{22}^{*}-2 \beta \sigma_{x}^{2} \lambda_{23}^{*}+\beta^{2} \sigma_{x}^{4} \lambda_{33}^{*}\right)
$$

ficando assim provado o corolário.

Notamos que neste caso particular, onde o estimador de $\beta$ coincide tanto no modelo assimétrico como no modelo normal usual, a variância assintótica esta modificada pelo parâmetro de assimetria $\delta_{x}$, resultado que era esperado. 


\subsubsection{Razão de variâncias $k_{x}^{*}$ conhecida}

Baseado no estimador do modelo normal, e fazendo um análise similar ao da seção acima, definimos,

$$
\widehat{\beta}_{2}=\left(\frac{k_{x}^{*}+1}{k_{x}^{*}}\right) \frac{S_{Y X}}{S_{X X}} \quad \text { com } \quad k_{x}^{*}=\frac{\sigma_{x}^{* 2}}{\sigma_{u}^{* 2}} \quad \text { conhecida, }
$$

onde, $\sigma_{x}^{* 2}=\sigma_{x}^{2}\left(1-\frac{2}{\pi} \delta_{x}^{2}\right)$ e $\sigma_{u}^{* 2}=\sigma_{u}^{2}\left(1-\frac{2}{\pi} \delta_{u}^{2}\right)$. Sob normalidade, este estimador foi estudado por Bolfarine e Cordani (1983).

Pelos resultados assintóticos do Capítulo 4 temos que,

$$
\widehat{\beta}_{2}=\left(\frac{k_{x}^{*}+1}{k_{x}^{*}}\right) \frac{S_{Y X}}{S_{X X}} \stackrel{q c}{\rightarrow}\left(\frac{k_{x}^{*}+1}{k_{x}^{*}}\right) \frac{\sigma_{Y X}^{2}}{\sigma_{X X}^{2}}=\beta
$$

Logo, $\widehat{\beta}_{2}$ é um estimador consistente para $\beta$ considerando $k_{x}^{*}$ conhecida.

A seguir, apresentamos a distribuição assintótica de $\widehat{\beta}_{2}$.

Proposição 5.3. Seja $\mathbf{Z}_{i}$ como em (5.1), $i=1, \ldots, n$, e $\widehat{\beta}_{2}=\left(\frac{k_{x}^{*}+1}{k_{x}^{*}}\right) \frac{S_{Y X}}{S_{X X}}$ o estimador do parâmetro $\beta$, tal que $k_{x}^{*}=\frac{\sigma_{x}^{*}}{\sigma_{u}^{*}}$ é conhecida. Então,

$$
\sqrt{n}\left(\widehat{\beta}_{2}-\beta\right) \stackrel{d}{\rightarrow} N\left(0, \Omega_{2}\right)
$$

onde,

$$
\Omega_{2}=\frac{1}{\left(1-\frac{2}{\pi} \delta_{x}^{2}\right)^{2} \sigma_{x}^{4}}\left(\lambda_{22}-2 \beta\left(\frac{k_{x}^{*}+1}{k_{x}^{*}}\right) \lambda_{23}+\beta^{2}\left(\frac{k_{x}^{*}+1}{k_{x}^{*}}\right)^{2} \lambda_{33}\right)
$$

tal que,

$$
\begin{aligned}
& \lambda_{22}=\sigma_{X Y}^{4}+\sigma_{X Y}^{2} \sigma_{X X}^{2}+\beta^{2} \sigma_{x}^{4} \delta_{x}^{*}, \\
& \lambda_{23}=\sigma_{X Y}^{2} \sigma_{X X}^{2}+\beta \sigma_{x}^{4} \delta_{x}^{*}, \\
& \lambda_{33}=2 \sigma_{X X}^{4}+\beta^{4} \sigma_{x}^{4} \delta_{x}^{*}+\sigma_{u}^{4} \delta_{u}^{*},
\end{aligned}
$$

com,

$$
\begin{array}{ll}
\sigma_{Y Y}^{2}=\left(1-\frac{2}{\pi} \delta_{x}^{2}\right) \beta^{2} \sigma_{x}^{2}+\left(1-\frac{2}{\pi} \delta_{e}^{2}\right) \sigma_{e}^{2} & \delta_{x}^{*}=\frac{8}{\pi}(\pi-3) \delta_{x}^{4} \\
\sigma_{X X}^{2}=\left(1-\frac{2}{\pi} \delta_{x}^{2}\right) \sigma_{x}^{2}+\left(1-\frac{2}{\pi} \delta_{u}^{2}\right) \sigma_{u}^{2} & \delta_{e}^{*}=\frac{8}{\pi}(\pi-3) \delta_{e}^{4} \\
\sigma_{X Y}^{2}=\left(1-\frac{2}{\pi} \delta_{x}^{2}\right) \beta \sigma_{x}^{2} & \delta_{u}^{*}=\frac{8}{\pi}(\pi-3) \delta_{u}^{4}
\end{array}
$$

Demonstração. Usando os mesmos argumentos que na prova da Proposição 5.1, temos que,

$$
\sqrt{n}\left(f\left[\operatorname{vech}\left(\mathrm{S}_{Z}\right)\right]-f\left[\operatorname{vech}\left(\Sigma_{Z}\right)\right]\right) \stackrel{d}{\rightarrow} N\left(0, \Omega_{2}\right) .
$$

Definindo $f\left((x, y, z)^{T}\right)=\left(\frac{k_{x}^{*}+1}{k_{x}^{*}}\right) \frac{y}{z-\sigma_{u}^{*}}$, temos que $f\left[\operatorname{vech}\left(\mathbf{S}_{Z}\right)\right]=\widehat{\beta}_{2}$ e $f\left[\operatorname{vech}\left(\Sigma_{Z}\right)\right]=\beta$.

Por outro lado, $\Omega_{2}=\Pi \Lambda \Pi^{T}$, onde, $\Lambda$ é a matriz de covariâncias como em (5.4.) e 
$\Pi=\frac{\partial f}{\partial\left(v_{e c h}^{T} \Sigma_{Z}\right)}$.

Assim, se $f\left((x, y, z)^{T}\right)=\left(\frac{k_{x}^{*}+1}{k_{x}^{*}}\right) \frac{y}{z-\sigma_{u}^{*}}$, então, $\frac{\partial f}{\partial(x, y, z)}=\left(\frac{k_{x}^{*}+1}{k_{x}^{*}}\right) \frac{1}{z}\left(0,1,-\frac{y}{z}\right)$ e portanto,

Logo, temos que,

$$
\Pi=\frac{\partial f}{\partial\left(\operatorname{vech}^{T} \Sigma_{Z}\right)}=\left(\frac{k_{x}^{*}+1}{k_{x}^{*}}\right) \frac{1}{\sigma_{X X}^{2}}\left(0,1,-\frac{\sigma_{Y X}^{2}}{\sigma_{X X}^{2}}\right) .
$$

$$
\begin{aligned}
\Omega_{2} & =\left(\frac{k_{x}^{*}+1}{k_{x}^{*}}\right) \frac{1}{\sigma_{X X}^{2}}\left(0,1,-\frac{\sigma_{Y X}^{2}}{\sigma_{X X}^{2}}\right)\left(\begin{array}{ccc}
\lambda_{11} & \lambda_{12} & \lambda_{13} \\
\ldots & \lambda_{22} & \lambda_{23} \\
\ldots & \ldots & \lambda_{33}
\end{array}\right)\left(\frac{k_{x}^{*}+1}{k_{x}^{*}}\right) \frac{1}{\sigma_{X X}^{2}}\left(\begin{array}{c}
0 \\
1 \\
-\frac{\sigma_{Y X}^{2}}{\sigma_{X X}^{2}}
\end{array}\right) \\
& =\left(\frac{k_{x}^{*}+1}{k_{x}^{*}}\right)^{2} \frac{1}{\sigma_{X X}^{4}}\left(\lambda_{22}-2 \beta\left(\frac{k_{x}^{*}}{k_{x}^{*}+1}\right) \lambda_{23}+\beta^{2}\left(\frac{k_{x}^{*}}{k_{x}^{*}+1}\right)^{2} \lambda_{33}\right),
\end{aligned}
$$

onde,

$$
\left(\frac{k_{x}^{*}+1}{k_{x}^{*}}\right)^{2} \frac{1}{\sigma_{X X}^{4}}=\frac{\sigma_{x}^{*}+\sigma_{u}^{*}}{\sigma_{x}^{*}} \frac{1}{\sigma_{x}^{*}+\sigma_{u}^{*}}=\frac{1}{\sigma_{x}^{*}}=\frac{1}{\left(1-\frac{2}{\pi} \delta_{x}^{2}\right) \sigma_{x}^{2}},
$$

com $\lambda_{23}$ e $\lambda_{33}$ segundo as expressões em (5.4.). Deste modo fica provado a proposição.

Considerando o modelo (5.1), com $\mathbf{r}_{i}=\left(v_{i}, e_{i}, u_{i}\right)^{T} \stackrel{i i d}{\sim} S N I_{3}(\mathbf{0}, \Psi, \lambda)$, onde, $\Psi=\operatorname{diag}\left\{\sigma_{x}^{2}, \sigma_{e}^{2}, \sigma_{u}^{2}\right\}$ e $\boldsymbol{\lambda}=\left(\lambda_{x}, 0,0\right)^{T}$, temos que, $\sigma_{x}^{* 2}=\sigma_{x}^{2}\left(1-\frac{2}{\pi} \delta_{x}^{2}\right)$ e $\sigma_{u}^{* 2}=\sigma_{u}^{2}$.

Portanto, $k_{x}^{*}=\frac{\sigma_{x}^{2}}{\sigma_{u}^{2}}\left(1-\frac{2}{\pi} \delta_{x}^{2}\right)=k_{x}\left(1-\frac{2}{\pi} \delta_{x}^{2}\right)$, onde, $k_{x}=\frac{\sigma_{x}^{2}}{\sigma_{u}^{2}}$. Definindo $k_{x}^{* *}=k_{x}\left(1-\frac{2}{\pi} \delta_{x}^{2}\right)$, então, o estimador de $\beta$ é,

$$
\widehat{\beta}_{2}^{*}=\left(\frac{k_{x}^{* *}+1}{k_{x}^{* *}}\right) \frac{S_{Y X}}{S_{X X}} \quad \text { com } \quad k_{x}^{* *}=k_{x}\left(1-\frac{2}{\pi} \delta_{x}\right) \quad \text { conhecida. }
$$

Notamos, neste caso, que o estimador é modificado pelo parâmetro de assimetria $\delta_{x}$. Derivamos a distribuição assintótica de $\widehat{\beta}_{2}^{*}$ como corolário da proposição anterior.

Corolario 5.4. Seja $\mathbf{Z}_{i}$ como em (5.1), $i=1, \ldots, n$, onde assumimos que, $\lambda_{e}=\lambda_{u}=0$, ou seja, $\delta_{e}=\delta_{u}=0$. Então o estimador do parâmetro $\beta$ é dado em (5.6), com

$$
\sqrt{n}\left(\widehat{\beta_{2}^{*}}-\beta\right) \stackrel{d}{\rightarrow} N\left(0, \Omega_{2}^{*}\right)
$$

onde,

tal que,

$$
\Omega_{2}^{*}=\frac{1}{\left(1-\frac{2}{\pi} \delta_{x}^{2}\right)^{2} \sigma_{x}^{4}}\left(\lambda_{22}^{*}-2 \beta\left(\frac{k_{x}^{* *}+1}{k_{x}^{* *}}\right) \lambda_{23}^{*}+\beta^{2}\left(\frac{k_{x}^{* *}+1}{k_{x}^{* *}}\right)^{2} \lambda_{33}^{*}\right),
$$

$$
\lambda_{22}^{*}=\sigma_{X Y}^{* 4}+\sigma_{X Y}^{* 2} \sigma_{X X}^{* 2}+\beta^{2} \sigma_{x}^{4} \delta_{x}^{*}, \quad \lambda_{23}^{*}=\sigma_{X Y}^{* 2} \sigma_{X X}^{* 2}+\beta \sigma_{x}^{4} \delta_{x}^{*}, \quad \lambda_{33}^{*}=2 \sigma_{X X}^{* 4}+\beta^{4} \sigma_{x}^{4} \delta_{x}^{*},
$$

com,

$$
\sigma_{Y Y}^{* 2}=\left(1-\frac{2}{\pi} \delta_{x}^{2}\right) \beta^{2} \sigma_{x}^{2}+\sigma_{e}^{2}, \quad \sigma_{X X}^{* 2}=\left(1-\frac{2}{\pi} \delta_{x}^{2}\right) \sigma_{x}^{2}+\sigma_{u}^{2}, \quad \sigma_{X Y}^{2 *}=\left(1-\frac{2}{\pi} \delta_{x}^{2}\right) \beta \sigma_{x}^{2},
$$

e $\delta_{x}^{*}=\frac{8}{\pi}(\pi-3) \delta_{x}^{4}$.

Ozán, N. S. 
Demonstração. Analogamente ao provado no Corolário 5.2, sob a hipótese $\delta_{e}=\delta_{u}=0$, temos as mesmas componentes para $\Sigma_{Z}$ e $\Lambda$. Portanto, usando o resultado da Proposição 5.3 , temos que a variância assintótica para $\widehat{\beta}_{2}^{*}$,

$$
\Omega_{2}^{*}=\frac{1}{\left(1-\frac{2}{\pi} \delta_{x}^{2}\right)^{2} \sigma_{x}^{4}}\left(\lambda_{22}^{*}-2 \beta\left(\frac{k_{x}^{* *}+1}{k_{x}^{* *}}\right) \lambda_{23}^{*}+\beta^{2}\left(\frac{k_{x}^{*}+1}{k_{x}^{* *}}\right)^{2} \lambda_{33}^{*}\right),
$$

com $k_{x}^{* *}$ dada em (5.6), ficando assim provado o corolário.

Observando, também neste caso, a influência do parâmetro de assimetria na variância assintótica do estimador de $\beta$.

\subsubsection{Razão de variâncias $k_{e}^{*}$ conhecida}

Baseado no estimador do modelo normal, e fazendo um análise similar ao da seção 5.2.2, temos que,

$$
\widehat{\beta}_{3}=\frac{S_{Y Y}-k_{e}^{*} S_{X X}-\left[\left(S_{Y Y}-k_{e}^{*} S_{X X}\right)^{2}+4 S_{Y X}^{2}\right]^{\frac{1}{2}}}{2 S_{Y X}}, \quad \text { com } \quad k_{e}^{*}=\frac{\sigma_{e}^{* 2}}{\sigma_{u}^{* 2}} \quad \text { conhecida, }
$$

onde, $\sigma_{e}^{* 2}=\sigma_{e}^{2}\left(1-\frac{2}{\pi} \delta_{e}^{2}\right)$ e $\sigma_{u}^{* 2}=\sigma_{u}^{2}\left(1-\frac{2}{\pi} \delta_{u}^{2}\right)$.

Pelos resultados assintóticos do Capítulo 4 temos que,

$$
\widehat{\beta}_{3}=\frac{S_{Y Y}-k_{e}^{*} S_{X X}-\left[\left(S_{Y Y}-k_{e}^{*} S_{X X}\right)^{2}+4 S_{Y X}^{2}\right]^{\frac{1}{2}}}{2 S_{Y X}} \stackrel{q c}{\rightarrow} \quad \frac{\sigma_{Y Y}-k_{e}^{*} \sigma_{X X}-\left[\left(\sigma_{Y Y}-k_{e}^{*} \sigma_{X X}\right)^{2}+4 \sigma_{Y X}^{2}\right]^{\frac{1}{2}}}{2 \sigma_{Y X}}=\beta .
$$

Logo, $\widehat{\beta}_{3}$ é um estimador consistente para $\beta$ considerando $k_{e}^{*}$ conhecida.

A seguir, apresentamos a distribuição assintótica de $\widehat{\beta}_{3}$.

Proposição 5.5. Seja $\mathbf{Z}_{i}$ como em (5.1), $i=1, \ldots, n$, e $\widehat{\beta}_{3}$ o estimador do parâmetro $\beta$ proposto em (5.7), então,

onde,

$$
\sqrt{n}\left(\widehat{\beta_{3}}-\beta\right) \stackrel{d}{\rightarrow} N\left(0, \Omega_{3}\right)
$$

$$
\Omega_{3}=a^{2} \lambda_{11}+2 a b \lambda_{12}+2 a c \lambda_{13}+b^{2} \lambda_{22}+2 b c \lambda_{23}+c^{2} \lambda_{33},
$$

com, $\lambda_{i j}, i, j=1,2,3$, dados em (5.4), e

$$
\begin{aligned}
a & =\frac{1+\left(\sigma_{Y Y}^{2}-k_{e}^{*} \sigma_{X X}^{2}\right)\left[\left(\sigma_{Y Y}^{2}-k_{e}^{*} \sigma_{X X}^{2}\right)^{2}+4 \sigma_{Y X}^{4}\right]^{-\frac{1}{2}}}{2 \sigma_{Y X}^{2}} \\
b & =\frac{-\sigma_{Y Y}^{2}+k_{e}^{*} \sigma_{X X}^{2}-\left[\left(\sigma_{Y Y}^{2}-k_{e}^{*} \sigma_{X X}\right)^{4}+4 \sigma_{Y X}^{4}\right]^{-\frac{1}{2}}}{2 \sigma_{Y X}^{4}}+\left[\left(\sigma_{Y Y}^{2}-k_{e}^{*} \sigma_{X X}^{2}\right)^{2}+4 \sigma_{Y X}^{4}\right]^{\frac{1}{2}}, \\
c & =\frac{-k_{e}^{*}+\left(\sigma_{Y Y}^{2}-k_{e}^{*} \sigma_{X X}^{2}\right) k_{e}^{*}\left[\left(\sigma_{Y Y}^{2}-k_{e}^{*} \sigma_{X X}^{2}\right)^{2}+4 \sigma_{Y X}^{4}\right]^{-\frac{1}{2}}}{2 \sigma_{Y X}^{2}}
\end{aligned}
$$

com,

$$
\begin{aligned}
\sigma_{Y Y}^{2} & =\left(1-\frac{2}{\pi} \delta_{x}^{2}\right) \beta^{2} \sigma_{x}^{2}+\left(1-\frac{2}{\pi} \delta_{e}^{2}\right) \sigma_{e}^{2} \\
\sigma_{X X}^{2} & =\left(1-\frac{2}{\pi} \delta_{x}^{2}\right) \sigma_{x}^{2}+\left(1-\frac{2}{\pi} \delta_{u}^{2}\right) \sigma_{u}^{2} \\
\sigma_{X Y}^{2} & =\left(1-\frac{2}{\pi} \delta_{x}^{2}\right) \beta \sigma_{x}^{2}
\end{aligned}
$$

Ozán, N. S. 
Demonstração. Usando os mesmos argumentos que na prova da Proposição 5.1, temos que,

$$
\sqrt{n}\left(f\left[\operatorname{vech}\left(\mathbf{S}_{Z}\right)\right]-f\left[\operatorname{vech}\left(\Sigma_{Z}\right)\right]\right) \stackrel{d}{\rightarrow} N\left(0, \Omega_{3}\right),
$$

onde, $\Omega_{3}=\Pi \Lambda \Pi^{T}$, com $\Lambda$ é a matriz de covariâncias como em (5.4.) e $\Pi=\frac{\partial f}{\partial\left(v_{e c h} \Sigma_{Z}\right)}$.

Se $f\left((x, y, z)^{T}\right)=\frac{x-k_{e} z+\left[\left(x k_{e} z\right)^{2}+4 y^{2}\right]^{\frac{1}{2}}}{2 y}$, então, $f\left[\operatorname{vech}\left(\mathbf{S}_{Z}\right)\right]=\widehat{\beta}_{12}$ e $f\left[\operatorname{vech}\left(\boldsymbol{\Sigma}_{Z}\right)\right]=\beta$ e

$$
\sqrt{n}\left(\widehat{\beta}_{3}-\beta\right) \stackrel{d}{\rightarrow} N\left(0, \Omega_{3}\right)
$$

Seja $\Pi=\frac{\partial f}{\partial\left(v e c h^{T} \Sigma_{Z}\right)}=(a, b, c)$, com,

$$
\begin{aligned}
& a=\frac{\partial f}{\partial \sigma_{Y Y}^{2}}=\frac{1+\left(\sigma_{Y Y}^{2}-k_{e}^{*} \sigma_{X X}^{2}\right)\left[\left(\sigma_{Y Y}^{2}-k_{e}^{*} \sigma_{X X}^{2}\right)^{2}+4 \sigma_{Y X}^{4}\right]^{-\frac{1}{2}}}{2 \sigma_{Y X}^{2}}, \\
& b=\frac{\partial f}{\partial \sigma_{Y X}^{2}}=\frac{-\sigma_{Y Y}^{2}+k_{e}^{*} \sigma_{X X}^{2}-\left[\left(\sigma_{Y Y}^{2}-k_{e}^{*} \sigma_{X X}\right)^{4}+4 \sigma_{Y X}^{4}\right]^{-\frac{1}{2}}}{2 \sigma_{Y X}^{4}}+\left[\left(\sigma_{Y Y}^{2}-k_{e}^{*} \sigma_{X X}^{2}\right)^{2}+4 \sigma_{Y X}^{4}\right]^{\frac{1}{2}}, \\
& c=\frac{\partial f}{\partial \sigma_{X X}^{2}}=\frac{-k_{e}^{*}+\left(\sigma_{Y Y}^{2}-k_{e}^{*} \sigma_{X X}^{2}\right) k_{e}^{*}\left[\left(\sigma_{Y Y}^{2}-k_{e}^{*} \sigma_{X X}^{2}\right)^{2}+4 \sigma_{Y X}^{4}\right]^{-\frac{1}{2}}}{2 \sigma_{Y X}^{2}},
\end{aligned}
$$

onde, $\sigma_{Y Y}^{2}, \sigma_{Y X}^{2}$ e $\sigma_{X X}^{2}$ são as componentes da matriz $\Sigma_{Z}$.

Portanto, temos que,

$$
\begin{aligned}
\Omega_{3} & =(a, b, c)\left(\begin{array}{ccc}
\lambda_{11} & \lambda_{12} & \lambda_{13} \\
\ldots & \lambda_{22} & \lambda_{23} \\
\ldots & \ldots & \lambda_{33}
\end{array}\right)\left(\begin{array}{c}
a \\
b \\
c
\end{array}\right) \\
& =a^{2} \lambda_{11}+2 a b \lambda_{12}+2 a c \lambda_{13}+b^{2} \lambda_{22}+2 b c \lambda_{23}+c^{2} \lambda_{33} .
\end{aligned}
$$

Deste modo fica provado a proposição.

Considerando o modelo (5.1), com $\mathbf{r}_{i}=\left(v_{i}, e_{i}, u_{i}\right)^{T} \stackrel{i i d}{\sim} S N I_{3}(0, \Psi, \lambda)$, onde, $\Psi=\operatorname{diag}\left\{\sigma_{x}^{2}, \sigma_{e}^{2}, \sigma_{u}^{2}\right\}$ e $\lambda=\left(\lambda_{x}, 0,0\right)^{T}$, temos que, $\sigma_{e}^{* 2}=\sigma_{e}^{2}$ e $\sigma_{u}^{* 2}=\sigma_{u}^{2}$.

De modo que, $k_{e}^{*}=\frac{\sigma_{e}^{2}}{\sigma_{u}^{2}}=k_{e}$, neste caso, o estimador não é modificado pelo parâmetro de assimetria $\delta_{x}$ e o estimador para $\beta$ é:

$$
\widehat{\beta}_{3}^{*}=\frac{S_{Y Y}-k_{e} S_{X X}-\left[\left(S_{Y Y}-k_{e} S_{X X}\right)^{2}+4 S_{Y X}^{2}\right]^{\frac{1}{2}}}{2 S_{Y X}}, \quad \text { com } \quad k_{e}=\frac{\sigma_{e}^{2}}{\sigma_{u}^{2}} \quad \text { conhecida. }
$$

Corolario 5.6. Seja $\mathrm{Z}_{i}$ como em (5.1), $i=1, \ldots, n$, onde assumimos que, $\lambda_{e}=\lambda_{u}=0$, ou seja, $\delta_{e}=\delta_{u}=0$. Então o estimador do parâmetro $\beta$ é dado em (5.8), com

$$
\sqrt{n}\left(\widehat{\beta}_{3}^{*}-\beta\right) \stackrel{d}{\rightarrow} N\left(0, \Omega_{3}^{*}\right)
$$

onde,

$$
\Omega_{3}^{*}=a^{* 2} \lambda_{11}^{*}+2 a^{*} b^{*} \lambda_{12}^{*}+2 a^{*} c^{*} \lambda_{13}^{*}+b^{* 2} \lambda_{22}^{*}+2 b^{*} c^{*} \lambda_{23}^{*}+c^{* 2} \lambda_{33}^{*},
$$

Ozán, N. S. 
com,

$$
\begin{aligned}
a^{*} & =\frac{1+\left(\sigma_{Y Y}^{* 2}-k_{e} \sigma_{X X}^{* 2}\right)\left[\left(\sigma_{Y Y}^{* 2}-k_{e} \sigma_{X X}^{* 2}\right)^{2}+4 \sigma_{Y X}^{* 4}\right]^{-\frac{1}{2}}}{2 \sigma_{Y X}^{* 2}}, \\
b^{*} & =\frac{-\sigma_{Y Y}^{* 2}+k_{e} \sigma_{X X}^{* 2}-\left[\left(\sigma_{Y Y}^{* 2}-k_{e} \sigma_{X X}\right)^{* 4}+4 \sigma_{Y X}^{* 4}\right]^{-\frac{1}{2}}}{2 \sigma_{Y X}^{* 4}}+\left[\left(\sigma_{Y Y}^{* 2}-k_{e} \sigma_{X X}^{* 2}\right)^{2}+4 \sigma_{Y X}^{* 4}\right]^{\frac{1}{2}}, \\
c^{*} & =\frac{-k_{e}+\left(\sigma_{Y Y}^{* 2}-k_{e} \sigma_{X X}^{* 2}\right) k_{e}\left[\left(\sigma_{Y Y}^{* 2}-k_{e} \sigma_{X X}^{* 2}\right)^{2}+4 \sigma_{Y X}^{* 4}\right]^{-\frac{1}{2}}}{2 \sigma_{Y X}^{* 2}},
\end{aligned}
$$

$$
\begin{array}{ll}
\lambda_{11}^{*}=2 \sigma_{Y Y}^{* 4}+\beta^{4} \sigma_{x}^{4} \delta_{x}^{*}, & \lambda_{22}^{*}=\sigma_{X Y}^{* 4}+\sigma_{X Y}^{* 2} \sigma_{X X}^{* 2}+\beta^{2} \sigma_{x}^{4} \delta_{x}^{*}, \\
\lambda_{12}^{*}=2 \sigma_{Y Y}^{* 2} \sigma_{X Y}^{* 2}+\beta^{3} \sigma_{x}^{4} \delta_{x}^{*}, & \lambda_{23}^{*}=\sigma_{X Y}^{* 2} \sigma_{X X}^{* 2}+\beta \sigma_{x}^{4} \delta_{x}^{*}, \\
\lambda_{13}^{*}=2 \sigma_{X Y}^{* 4}+\beta^{2} \sigma_{x}^{4} \delta_{x}^{*}, & \lambda_{33}^{*}=2 \sigma_{X X}^{* 4}+\beta^{4} \sigma_{x}^{4} \delta_{x}^{*},
\end{array}
$$

$$
\sigma_{Y Y}^{* 2}=\left(1-\frac{2}{\pi} \delta_{x}^{2}\right) \beta^{2} \sigma_{x}^{2}+\sigma_{e}^{2}, \quad \sigma_{X X}^{* 2}=\left(1-\frac{2}{\pi} \delta_{x}^{2}\right) \sigma_{x}^{2}+\sigma_{u}^{2}, \quad \sigma_{X Y}^{2 *}=\left(1-\frac{2}{\pi} \delta_{x}^{2}\right) \beta \sigma_{x}^{2},
$$

$e \delta_{x}^{*}=\frac{8}{\pi}(\pi-3) \delta_{x}^{4}$.

Demonstração. Sob a hipótese $\delta_{e}=\delta_{u}=0$ e usando o resultado da Proposição 5.5 temos provado o corolário.

Observando, neste caso que os estimadores para o parâmetro $\beta$ no modelo simétrico e assimétrico coinciden, mas temos a influência do parâmetro de assimetria na variância assintótica.

\subsection{Estimação de Maxima Verossimilhança}

Seja $\mathbf{Z}_{1}, \ldots, \mathbf{Z}_{n}$ uma amostra aleatória de $n$ observações independentes sob as suposições da expressão (4.1). Conforme vimos na Seção 4.2, a função de densidade marginal de $\mathbf{Z}_{i}=\left(Y_{i}, X_{i}\right)^{T}, i=1, \ldots, n$, é dada por,

$$
f_{\mathbf{Z}}\left(\mathbf{z}_{i} \mid \theta, \lambda\right)=2^{3}|\Sigma|^{-\frac{1}{2}} \phi_{2}\left(\Sigma^{-\frac{1}{2}}\left(\mathbf{z}_{i}-\boldsymbol{\mu}\right)\right) \Phi_{3}\left(\Delta_{*} \Sigma^{-\frac{1}{2}}\left(\mathbf{z}_{i}-\boldsymbol{\mu}\right) \mid \mathbf{0},\left(\Psi+\frac{\Delta \mathbf{b b}^{T} \Delta}{\mathbf{b}^{T} \Psi^{-1} \mathbf{b}}\right)\right)
$$

onde,

- $\boldsymbol{\theta}=\left(\alpha, \beta, \mu_{x}, \sigma_{x}^{2}, \sigma_{e}^{2}, \sigma_{u}^{2}\right)^{T}$ e $\boldsymbol{\lambda}=\left(\lambda_{x}, \lambda_{e}, \lambda_{u}\right)^{T}$,

- $\boldsymbol{\mu}=\left(\begin{array}{c}\alpha+\beta \mu_{x} \\ \mu_{x}\end{array}\right)$ e $\Sigma=\left(\begin{array}{cc}\beta^{2} \sigma_{x}^{2}+\sigma_{e}^{2} & \beta \sigma_{x}^{2} \\ \beta \sigma_{x}^{2} & \sigma_{x}^{2}+\sigma_{u}^{2}\end{array}\right)$

- $\Delta_{*}=\Delta \Psi \mathrm{B}^{T} \Sigma^{-\frac{1}{2}}, \Delta=\operatorname{Diag}\left\{\lambda_{x}, \lambda_{e}, \lambda_{u}\right\}, \mathrm{b}=(-1, \beta, 1)^{T}$.

Para derivar o estimadores de máxima verossimilhança temos que,

$$
L\left(\boldsymbol{\theta}, \lambda \mid \mathbf{z}_{1}, \ldots, \mathbf{z}_{n}\right)=\prod_{i=n}^{n} f_{Z}\left(\mathbf{z}_{i} \mid \boldsymbol{\theta}, \lambda\right)
$$

Ozán, N. S. 
e sendo $\boldsymbol{\theta}^{*}=(\boldsymbol{\theta}, \boldsymbol{\lambda})^{T}$, temos que,

$$
\begin{aligned}
\ell\left(\boldsymbol{\theta}^{*}\right)=\log \left[L\left(\boldsymbol{\theta}, \lambda \mid \mathbf{z}_{1}, \ldots, \mathbf{z}_{n}\right)\right] & =\log \left[\prod_{i=n}^{n} f_{Z}\left(\mathbf{z}_{i} \mid \boldsymbol{\theta}, \boldsymbol{\lambda}\right)\right] \\
& =\sum_{i=n}^{n} \log \left[f_{Z}\left(\mathbf{z}_{i} \mid \boldsymbol{\theta}, \boldsymbol{\lambda}\right)\right] \\
& =\sum_{i=n}^{n} \log \left[2^{3}|\boldsymbol{\Sigma}|^{-\frac{1}{2}} \phi_{2}\left(\boldsymbol{\Sigma}^{-\frac{1}{2}}\left(\mathbf{z}_{i}-\boldsymbol{\mu}\right)\right) \Phi_{3}\left(\boldsymbol{\Delta}_{*} \boldsymbol{\Sigma}^{-\frac{1}{2}}\left(\mathbf{z}_{i}-\boldsymbol{\mu}\right)\right)\right] .
\end{aligned}
$$

Assim,

$$
\begin{aligned}
\ell\left(\boldsymbol{\theta}^{*}\right)= & 3 n \log (2)-\frac{n}{2} \log [|\boldsymbol{\Sigma}|]+\sum_{i=n}^{n} \log \left[\phi_{2}\left(\boldsymbol{\Sigma}^{-\frac{1}{2}}\left(\mathbf{z}_{i}-\boldsymbol{\mu}\right)\right)\right]+\sum_{i=n}^{n} \log \left[\Phi_{3}\left(\boldsymbol{\Delta}_{*} \boldsymbol{\Sigma}^{-\frac{1}{2}}\left(\mathbf{z}_{i}-\boldsymbol{\mu}\right)\right)\right] \\
= & 3 n \log (2)-\frac{n}{2} \log [|\boldsymbol{\Sigma}|]+n \log \left[\frac{1}{2 \pi}\right]-\frac{1}{2} \sum_{i=n}^{n}\left(\mathbf{z}_{i}-\boldsymbol{\mu}\right)^{T} \boldsymbol{\Sigma}^{-1}\left(\mathbf{z}_{i}-\boldsymbol{\mu}\right)+ \\
& +\sum_{i=n}^{n} \log \left[\Phi_{3}\left(\boldsymbol{\Delta}_{*} \boldsymbol{\Sigma}^{-\frac{1}{2}}\left(\mathbf{z}_{i}-\boldsymbol{\mu}\right)\right)\right] .
\end{aligned}
$$

De modo que,

$$
\ell\left(\boldsymbol{\theta}^{*}\right) \propto-\frac{n}{2} \log [|\Sigma|]-\frac{1}{2} \sum_{i=n}^{n}\left(\mathbf{z}_{i}-\boldsymbol{\mu}\right)^{T} \Sigma^{-1}\left(\mathbf{z}_{i}-\boldsymbol{\mu}\right)+\sum_{i=n}^{n} \log \left[\Phi_{3}\left(\boldsymbol{\Delta}_{*} \boldsymbol{\Sigma}^{-\frac{1}{2}}\left(\mathbf{z}_{i}-\boldsymbol{\mu}\right)\right)\right] .
$$

Então,

$$
\begin{aligned}
\frac{\partial \ell\left(\boldsymbol{\theta}^{*}\right)}{\partial \boldsymbol{\theta}^{*}}= & -\frac{n}{2|\boldsymbol{\Sigma}|} \frac{\partial|\boldsymbol{\Sigma}|}{\partial \boldsymbol{\theta}^{*}}-\frac{1}{2} \frac{\partial}{\partial \boldsymbol{\theta}^{*}} \sum_{i=n}^{n}\left(\mathbf{z}_{i}-\boldsymbol{\mu}\right)^{T} \boldsymbol{\Sigma}^{-1}\left(\mathbf{z}_{i}-\boldsymbol{\mu}\right)+ \\
& +\sum_{i=n}^{n}\left(\frac{\phi_{3}\left(\boldsymbol{\Delta}_{*} \boldsymbol{\Sigma}^{-\frac{1}{2}}\left(\mathbf{z}_{i}-\boldsymbol{\mu}\right)\right)}{\Phi_{3}\left(\Delta_{*} \boldsymbol{\Sigma}^{-\frac{1}{2}}\left(\mathbf{z}_{i}-\boldsymbol{\mu}\right)\right)}\right) \frac{\partial}{\partial \boldsymbol{\theta}^{*}}\left[\boldsymbol{\Delta}_{*} \boldsymbol{\Sigma}^{-\frac{1}{2}}\left(\mathbf{z}_{i}-\boldsymbol{\mu}\right)\right]
\end{aligned}
$$

Definindo $K_{3 i}\left(\boldsymbol{\theta}^{*}\right)=\frac{\phi_{3}\left(w_{i}\left(\boldsymbol{\theta}^{*}\right)\right)}{\Phi_{3}\left(w_{i}\left(\boldsymbol{\theta}^{*}\right)\right)}, \operatorname{com} w_{i}\left(\boldsymbol{\theta}^{*}\right)=\boldsymbol{\Delta}_{*} \Sigma^{-\frac{1}{2}}\left(\mathbf{z}_{i}-\boldsymbol{\mu}\right), i=1, \ldots, n$, então, escrevemos a expressão (5.10) como,

$$
\frac{\partial \ell\left(\boldsymbol{\theta}^{*}\right)}{\partial \boldsymbol{\theta}^{*}}=-\frac{n}{2|\boldsymbol{\Sigma}|} \frac{\partial|\boldsymbol{\Sigma}|}{\partial \boldsymbol{\theta}^{*}}-\frac{1}{2} \frac{\partial}{\partial \boldsymbol{\theta}^{*}} \sum_{i=n}^{n}\left(\mathbf{z}_{i}-\boldsymbol{\mu}\right)^{T} \Sigma^{-1}\left(\mathbf{z}_{i}-\boldsymbol{\mu}\right)+\sum_{i=n}^{n} K_{3 i}\left(\boldsymbol{\theta}^{*}\right) \frac{\partial w_{i}\left(\boldsymbol{\theta}^{*}\right)}{\partial \boldsymbol{\theta}^{*}} .
$$

Então, os estimadores de máxima verossimilhança são a solução do sistema de equações dado por $\frac{\partial \ell\left(\boldsymbol{\theta}^{*}\right)}{\partial \boldsymbol{\theta}^{*}}=0$. Por exemplo, para calcular o estimador de $\lambda$, a equação a resolver é

$$
\frac{\partial \ell\left(\boldsymbol{\theta}^{*}\right)}{\partial \boldsymbol{\lambda}}=\sum_{i=n}^{n} \frac{\partial}{\partial \lambda}\left\{K_{3 i}\left(\boldsymbol{\theta}^{*}\right) \frac{\partial w_{i}\left(\boldsymbol{\theta}^{*}\right)}{\partial \boldsymbol{\theta}^{*}}\right\}=0 .
$$

Ozán, N. S. 
É claro que não podemos derivar uma expressão fechada para as equações de estimação, tendo que resolver mediante algum método iterativo o sistema de equações.

Notamos que os dois primeiros termos em (5.10) correspondem ao sistema de equações de estimação do modelo normal usual, o fato de ter assimetria adiciona o terceiro termo na equação. Portanto, quando o parâmetro de assimetria é zero temos que $\boldsymbol{\theta}^{*}=\boldsymbol{\theta}$ e o problema de estimação se reduz ao caso já conhecido nos modelos normal e elíptico desenvolvidos na literatura (Fuller (1987), Bolfarine e Cordani (1993), Arellano-Valle, Bolfarine e Vilca-Labra (1996)).

Discutimos a seguir o caso particular em que assumimos assimetria só na covariável não observada $x$. Seja $\mathbf{Z}_{i}$ como em (5.1), tal que, $\mathbf{r}_{i}=\left(v_{i}, e_{i}, u_{i}\right)^{T} \stackrel{i n d}{\sim} S N I_{3}(\mathbf{0}, \Psi, \lambda)$, onde $\Psi=$ $\operatorname{diag}\left\{\sigma_{x}^{2}, \sigma_{e}^{2}, \sigma_{u}^{2}\right\}$ e $\lambda=\left(\lambda_{x}, 0,0\right)^{T}$. A função de densidade marginal para $\mathbf{Z}_{i}=\left(Y_{i}, X_{i}\right)^{T}$ segundo (4.16), é dada por,

$$
f_{\mathbf{Z}}\left(\mathbf{z}_{i}\right)=2|\Sigma|^{-\frac{1}{2}} \phi_{2}\left(\boldsymbol{\Sigma}^{-\frac{1}{2}}\left(\mathbf{z}_{i}-\boldsymbol{\mu}\right)\right) \Phi\left(\Delta_{* *} \boldsymbol{\Sigma}^{-\frac{1}{2}}\left(\mathbf{z}_{i}-\boldsymbol{\mu}\right)\right)
$$

com,

$$
\begin{aligned}
& \text { - } \Delta_{* *}=d \mathbf{e}_{1} \mathbf{b}_{1}^{T} \Sigma^{-\frac{1}{2}}, \\
& \text { - } d=\lambda_{x} \sigma_{x}^{2}\left(\sigma_{x}^{2}+\frac{\lambda_{x}^{2}}{\mathbf{b}^{T} \Psi^{-1} \mathbf{b}}\right)^{-\frac{1}{2}}, \mathbf{b}=(-1, \beta, 1)^{T}, \mathbf{b}_{1}=(\beta, 1)^{T} \text { e } \mathbf{e}_{1}=(1,0,0)^{T} .
\end{aligned}
$$

O vetor de parâmetros neste caso agora é $\boldsymbol{\theta}^{*}=\left(\alpha, \beta, \mu_{x}, \sigma_{x}^{2}, \sigma_{e}^{2}, \sigma_{u}^{2}, \lambda_{x}\right)^{T}$, e a função de log-verossimilhança é,

$$
\ell\left(\boldsymbol{\theta}^{*}\right)=-\frac{n}{2} \log [|\boldsymbol{\Sigma}|]-\frac{1}{2} \sum_{i=n}^{n}\left(\mathbf{z}_{i}-\boldsymbol{\mu}\right)^{T} \boldsymbol{\Sigma}^{-1}\left(\mathbf{z}_{i}-\boldsymbol{\mu}\right)+\sum_{i=n}^{n} \log \left[\Phi\left(\boldsymbol{\Delta}_{* *} \boldsymbol{\Sigma}^{-\frac{1}{2}}\left(\mathbf{z}_{i}-\boldsymbol{\mu}\right)\right)\right](
$$

Considerando, $K_{1 i}\left(\boldsymbol{\theta}^{*}\right)=\frac{\phi\left(w_{i}\left(\boldsymbol{\theta}^{*}\right)\right)}{\Phi\left(w_{i}\left(\boldsymbol{\theta}^{*}\right)\right)}$, agora com $w_{i}\left(\boldsymbol{\theta}^{*}\right)=\Delta_{* *} \boldsymbol{\Sigma}^{-\frac{1}{2}}\left(\mathbf{z}_{i}-\boldsymbol{\mu}\right), i=1, \ldots, n$, temos que a expressão para a derivada da função de log-verossimilhança é,

$$
\frac{\partial \ell\left(\boldsymbol{\theta}^{*}\right)}{\partial \boldsymbol{\theta}^{*}}=-\frac{n}{2|\Sigma|} \frac{\partial|\Sigma|}{\partial \boldsymbol{\theta}^{*}}-\frac{1}{2} \frac{\partial}{\partial \boldsymbol{\theta}^{*}} \sum_{i=n}^{n}\left(\mathrm{z}_{i}-\boldsymbol{\mu}\right)^{T} \Sigma^{-1}\left(\mathbf{z}_{i}-\boldsymbol{\mu}\right)+\sum_{i=n}^{n} K_{1 i}\left(\boldsymbol{\theta}^{*}\right) \frac{\partial w_{i}\left(\boldsymbol{\theta}^{*}\right)}{\partial \boldsymbol{\theta}^{*}}
$$

Assim a dimensão do espaço de parâmetros foi reduzida, mas o problema de achar uma expressão fechada para os estimadores de máxima verossimilhança continua.

Conseqüentemente, obtemos os estimadores usando métodos de aproximação numérica como apresentamos na seguinte seção.

Ozán, N. S. 


\subsubsection{Estudo de simulação}

No estudo de simulação feito para analisar os estimadores de máxima verossimilhança consideramos a seguinte situação: a assimetria esta na covariável não observada $x$, isto é, assumimos o modelo $\mathbf{Z}_{i}$ como em (5.1), tal que o parâmetro de assimetria é $\boldsymbol{\lambda}=\left(\lambda_{x}, 0,0\right)^{T}$, $\lambda_{x} \neq 0$, com a função de densidade dada em (5. 12.). Para evitar a não identificabilidade do modelo, como já vimos na Seção 4.2, (Capítulo 4), consideramos a razão de variâncias $k_{e}=\frac{\sigma_{e}^{2}}{\sigma_{u}^{2}}$, conhecida. Sem perda de generalidade, assumimos $k_{e}=1$ o que implica que $\sigma_{e}^{2}=\sigma_{u}^{2}=\sigma^{2}$.

O vetor de parâmetros é $\theta^{*}=\left(\alpha, \beta, \mu_{x}, \sigma_{x}^{2}, \sigma^{2}, \lambda_{x}\right)^{T}$, e a função para maximizar é,

$$
\ell\left(\boldsymbol{\theta}^{*}\right)=-\frac{n}{2} \log [|\Sigma|]-\frac{1}{2} \sum_{i=n}^{n}\left(\mathbf{z}_{i}-\boldsymbol{\mu}\right)^{T} \boldsymbol{\Sigma}^{-1}\left(\mathbf{z}_{i}-\boldsymbol{\mu}\right)+\sum_{i=n}^{n} \log \left[\Phi\left(\Delta_{* *} \Sigma^{-\frac{1}{2}}\left(\mathbf{z}_{i}-\boldsymbol{\mu}\right)\right)\right]
$$

O modelo linear aditivo assimétrico com erros nas variáveis que consideramos no estudo tem como valores verdadeiros dos parâmetros os seguintes,

$$
\begin{array}{lll}
\alpha=1, & \beta=2, & \boldsymbol{\mu}_{x}=1 \\
\sigma_{x}^{2}=1, & \sigma_{e}^{2}=1, & \sigma_{u}^{2}=1 .
\end{array}
$$

No caso do parâmetro $\lambda_{x}$, no primeiro estudo tem como valor verdadeiro 3 , e no segundo estudo diferentes valores de $\lambda_{x}$.

Geramos um conjunto de dados $\mathbf{Z}_{i}$, segundo o modelo especificado acima, $i=1, \ldots, n$, com $e_{i} \stackrel{i i d}{\sim} S N(0,1,0)$ e $u_{i} \stackrel{i i d}{\sim} S N(0,1,0)$, o que equivale a gerar amostras normais $N(0,1)$. Para gerar $v_{i}^{*}=\frac{v_{i}}{\sigma_{x}} \stackrel{\text { iid }}{\sim} S N\left(0,1, \lambda_{x}\right)$, onde $v_{i}=x_{i}-\mu_{x}$, consideramos, $v_{i}^{*}=\delta_{x}\left|Y_{0}\right|+\left(1-\delta_{x}^{2}\right)^{\frac{1}{2}} Y_{1}$, com $Y_{0} \sim N(0,1), Y_{1} \sim N(0,1)$, independentes e $\delta_{x}=\frac{\lambda_{x}}{\left(1+\lambda^{2}\right)^{\frac{1}{2}}}$.

Logo para $\alpha=1$ e $\beta=2$ obtemos a mostra $\mathbf{Z}_{i}, i=1, \ldots, n$. No primeiro caso consideramos $n=50$, realizando depois uma analise para diferentes valores de $n$ e $\lambda_{x}$.

Os resultados obtidos são os seguinte.

1. Na Tabela 5.1, apresentamos os resultados da inferência do vetor de parâmetros $\theta^{*}=\left(\alpha, \beta, \mu_{x}, \sigma_{x}^{2}, \sigma^{2}, \lambda_{x}\right)^{T}$, para $n=50$ observações. A variância observada, simbolizada como $V_{o b s}\left[\widehat{\theta}^{*}\right]$, pode ser considerada como uma variância empírica, é obtida como a inversa da matriz de informação de Fisher observada, dividida por $n$. Se consideramos que a distribuição assintótica do parâmetro $\beta$ é normal, então, o intervalo observado para $\beta$ é $(1.026,2.691)$, com $95 \%$ de confiança.

Observamos, em geral, que a diferencia $\left|\widehat{\theta}^{*}-\theta^{*}\right|$ é pequena para quase todos os estimadores, o que indica que o método de estimação é bom. No que diz respeito as variâncias, notamos que só no caso da $\operatorname{Var}\left[\widehat{\lambda}_{x}\right]$ temos um valor um pouco maior. 
Tabela 5.1 Estimativas de $\boldsymbol{\theta}^{*}$

\begin{tabular}{lcccccc}
\hline \hline & $\widehat{\mu}_{x}$ & $\widehat{\beta}$ & $\widehat{\alpha}$ & $\widehat{\sigma}_{x}^{2}$ & $\widehat{\sigma}^{2}$ & $\widehat{\lambda}_{x}$ \\
& 1.06 & 1.86 & 1.35 & 1.10 & 0.81 & 1.92 \\
\hline$\left|\widehat{\theta^{*}}-\boldsymbol{\theta}^{*}\right|$ & 0.06 & 0.14 & 0.35 & 0.10 & 0.18 & 1.07 \\
$V_{\text {obs }}\left[\widehat{\theta}^{*}\right]$ & 0.19 & 0.18 & 0.65 & 0.57 & 0.03 & 4.73 \\
\hline \hline
\end{tabular}

O problema está na estimação do parâmetro de assimetria $\lambda_{x}$, onde notamos que a estimativa pode ter uma boa diferença com o verdadeiro valor do parâmetro, e um valor alto para a variância. A razão deste tipo de problema é devido a que o parâmetro a maximizar é parte do argumento de uma função logarítmica, como vemos em (5.13), a qual tem curvatura pequena na direção do parâmetro de assimetria, o que dificulta achar o máximo, sendo esta uma limitação do método de maximização. Contudo, como mencionam Azzalini e Capitanio (1999) a estimação dos parâmetros de locação e escala são bastante estáveis apesar da variabilidade presente na estimação do parâmetro de assimetria. Portanto, é nosso interesse discutir, especificamente, a estimação do parâmetro $\beta$ usando o método de máxima verossimilhança, como fazemos no seguinte item.

2. Estudamos o comportamento do parâmetro $\beta$, gerando amostras de diversos tamanhos e para diferentes valores de $\lambda_{x}$, na Tabela 5.2 apresentamos os valores obtidos. Neste caso, observamos que se confirma a eficácia do método de máxima verossimilhança para estimar o parâmetro $\beta$ e suas propriedades de robustez, fato aprofundado no próximo estudo.

Tabela 5.2 Estimativas de $\beta$

\begin{tabular}{lllll}
\hline \hline & & $n$ & & \\
$\lambda_{x}$ & 30 & 50 & 80 & 100 \\
\hline 3 & 1.54 & 1.86 & 1.58 & 1.71 \\
2 & 1.49 & 1.91 & 2.53 & 1.51 \\
1 & 1.95 & 1.99 & 2.30 & 1.95 \\
\hline 0 & 2.21 & 2.07 & 2.60 & 1.97 \\
\hline-1 & 2.24 & 2.06 & 1.98 & 2.29 \\
-2 & 2.31 & 1.68 & 2.62 & 2.49 \\
-3 & 2.36 & 1.59 & 1.62 & 2.51 \\
\hline \hline
\end{tabular}

3. Analisamos o comportamento do estimador do parâmetro $\beta$, realizando um estudo 
de simulação Monte Carlo para k réplicas, isto é, geramos k amostras de tamanho $n=50$. Consideramos $k=10,50,100,500,1000$. Na Tabela 5.3, apresentamos os resultados obtidos no estudo, para o qual, geramos uma amostra de 50 observações independentes, realizando $\mathrm{k}$ réplicas desta amostra (por exemplo $\mathrm{k}=10$ ), obtemos para cada réplica o estimador do parâmetro $\beta$, sendo assim $\widehat{\beta}_{M}$ como a média dos estimadores de cada réplica. Calculamos a variância deste estimador, o viés e o erro quadrático médio para anlizar o comportamento do estimador na simulação. É clara a robustez do parâmetro $\beta$, embora seja obtida mediante um método numérico.

Tabela 5.3 Estudo de simulação para $\beta$

\begin{tabular}{cccccc}
\hline \hline$k$ & 10 & 50 & 100 & 500 & 1000 \\
\hline & & & & & \\
$\widehat{\beta}_{M}$ & 1.96 & 2.11 & 2.26 & 2.26 & 2.28 \\
$\operatorname{Var}\left[\widehat{\beta}_{M}\right]$ & 0.24 & 0.54 & 0.84 & 0.15 & 0.20 \\
$E\left[\widehat{\beta}_{M}-\beta\right]$ & -0.04 & 0.11 & 0.26 & 0.23 & 0.28 \\
$E Q M\left[\widehat{\beta}_{M}\right]$ & 0.24 & 0.55 & 0.91 & 0.98 & 0.27 \\
\hline \hline
\end{tabular}

Todo o estudo de simulação foi feito no linguagem de programação $O x$ usando a função MaxBFGS. Os detalhes do algoritmo interativo e programas utilizados são apresentados no Apêndice.

\subsection{Aplicação}

Analisamos a relação entre a taxa de mortalidade infantil e a proporção de pobres nos estados da União Federal do Brasil no ano 1999.

\subsubsection{Apresentação do problema}

O estudo da taxa de mortalidade infantil é de grande interesse na área da Saúde Pública. Esta taxa pode estar relacionada com diferentes indicadores sócio- econômicos, como por exemplo, a taxa de desemprego, a proporção de pobres o analfabetismo, etc. Ou com outro tipo de indicadores relacionados com as condições de vida da população.

Neste caso estudamos a relação da taxa de mortalidade infantil com a proporção de pobres, que consideramos como sendo linear, após de um estudo gráfico da relação entre as variáveis.

Especificamos o significado das variáveis observadas e a fonte de informação.

- Taxa de mortalidade infantil (TMI). Número de óbitos infantis (menores de 1 ano) 
por 1.000 nascidos vivos no Brasil, ano 1999.

Fontes: Sistema de Informações sobre Nascidos Vivos (SINASC). Sistema de Informações sobre Mortalidade (SIM), Ministerio de Saúde. IBGE.

- Proporção de pobres (PP). Estimativas demográficas Percentual da população em estado de pobreza, segundo Região do Brasil, ano 1999.

Fonte: IBGE. Pesquisa Nacional por Amostra de Domicílios (PNAD).

O conjunto de dados apresenta-se no final do capítulo na Tabela 5. 6, e também se encontra disponível em www.datasus.gov.br.

\subsubsection{Análise do problema}

A proporção de pobres é o percentual da população residente com renda familiar mensal per capita de até meio salário mínimo em determinado espaço geográfico no ano 1999. O percentual se calcula como a razão entre a população residente com renda familiar mensal per capita de até meio salário mínimo sobre a população residente (por 100). Na informação da renda familiar, não sempre é conhecido o valor real, já que em muitas situações as pessoas não declaram seu salário real ou não tem salário fixo (desempregados). Portanto, podemos considerar não conhecer o verdadeiro valor da renda familiar, o que justifica pensar em ajustar um modelo com erros nas variáveis.

Realizamos alguns gráficos para analisar a suposição de assimetria. Na figura 5.1, apresentamos o gráfico Box-Plot para a taxa de mortalidade infantil e a proporção de pobres, onde se observa uma assimetria positiva.

O gráfico de assimetria, na figura 5.2 , é formado pelos pares ordenados $\left(u_{i}, v_{i}\right)$, com $u_{i}=M d-x_{(i)}$ e $v_{i}=x_{(n+1-i)}-M d, i=1, \ldots, \frac{(n+1)}{2}$, onde $x_{(1)} \leq x_{(2)} \leq \ldots x_{(n)}$ são as estatísticas de ordem e $M d$ a mediana, nesta figura consideramos a variável proporção de pobres para realizar o gráfico. Observamos que a maioria dos pontos se encontram acima da linha de referencia $u=v$, isto significa que para determinado $u_{i}$ o correspondente $v_{i}$ é maior. Portanto, para a maioria dos pontos, a distância de cada $x_{(n+1-i)}$ à mediana é menor que a distancia de $x_{(i)}$ à mediana, e portanto há assimetria positiva. 
Figura 5.1 Gráfico Box-Plot

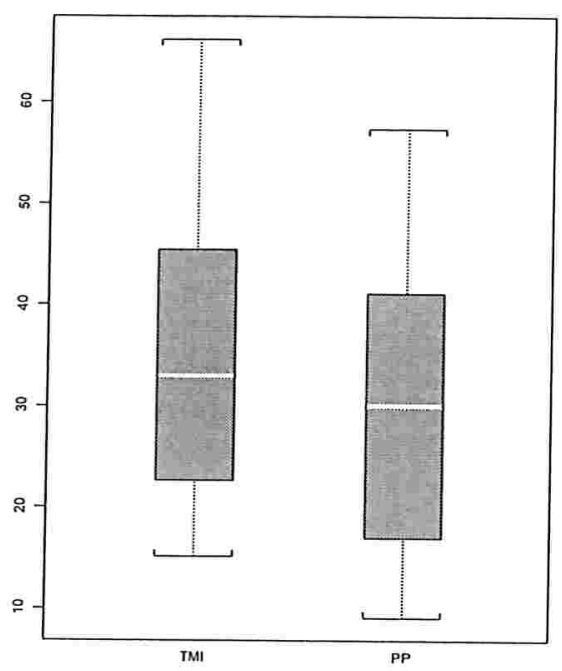

Figura 5.2 Gráfico de Assimetria

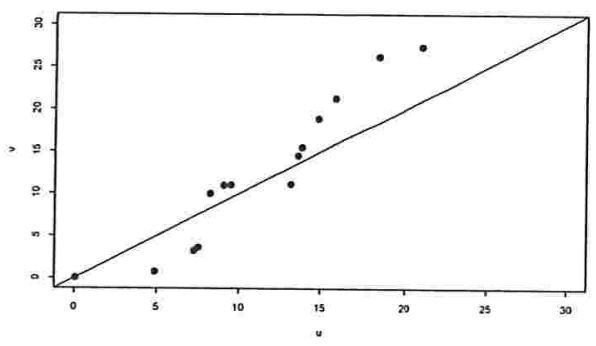

Ozán, N. S. 
Na tabela 5.1. apresentamos o resultado da estatística descritiva da taxa de mortalidade infantil e a proporção de pobres. Notamos na proporção de pobres, uma gram diferença entre o valor mínimo e o máximo, $9.09 \%$ e $57.39 \%$, e uma assimetria positiva.

Covariâncias amostrais: $S_{Y Y}=219.0479, S_{Y X}=178.9328$ e $S_{X X}=204.1487$.

Tabela 5.4 Estatística Descritiva

\begin{tabular}{lccccccccc}
\hline \hline & Min. & $1^{\circ}$ Q. & Média & Mediana & $3^{\circ}$ Q. & Máx. & D.S. & Assimetria & Curtose \\
\hline TMI & 15.10 & 23.75 & 35.39 & 32.96 & 45.41 & 66.13 & 14.8 & 0.45 & -0.88 \\
PP & 9.09 & 18.80 & 30.78 & 30.07 & 41.07 & 57.39 & 14.28 & 0.33 & -1.08 \\
\hline \hline
\end{tabular}

O coeficiente de correlação linear (0.846) indica que, há uma relação linear entre entre a taxa de mortalidade infantil e a proporção de pobres.

Assim, consideramos que,

- Existe uma relação linear entre as covariáveis estudadas, ou seja, entre a Taxa de Mortalidade Infantil (Y) e a Proporção de Pobres (X).

- A covariável, Proporção de Pobres (X), é medida com erros, pois não temos certeza do verdadeiro valor da renda familiar da população considerada.

- Fazendo uma descrição dos dados, observamos que há evidencia de assimetria.

- Estimativas para $\sigma_{u}^{2}$ e $\sigma_{e}^{2}$, são considerando os dados dos anos 1997 e 1998 como réplicas, e usando,

$$
\widehat{\sigma}_{e}^{2}=\frac{1}{2} S_{Y, 97-Y, 98}^{2}=14.25 \quad \widehat{\sigma}_{u}^{2}=\frac{1}{2} S_{X, 97-X, 98}^{2}=5.96,
$$

onde $S_{Y, 97-Y, 98}^{2}$ e $S_{X, 97-X, 98}^{2}$ são as variâncias amostrais da diferença das observações no ano 1997 e 1998. Logo, uma estimativa de $k_{e}=\frac{\sigma_{e}^{2}}{\sigma_{u}^{2}}$, é aproximadamente 2, $\left(\widehat{k}_{e}=\frac{\widehat{\sigma}_{e}^{2}}{\widehat{\sigma}_{u}^{2}}=2.39\right)$. Portanto, podemos considerar que $\sigma_{e}^{2}=2 \sigma_{u}^{2}$. Incluímos também no estudo a suposição de variâncias iguais, isto é, $\sigma_{e}^{2}=\sigma_{u}^{2}$, para comparar o comportamento dos estimadores.

Portanto, ajustamos o modelo normal assimétrico com erros nas variáveis com as suposições da seção anterior, onde no Modelo 1 consideramos $\sigma_{e}^{2}=\sigma_{u}^{2}$ e no Modelo 2 $\sigma_{e}^{2}=2 \sigma_{u}^{2}$.

Os resultados do ajuste se apresentam na Tabela 5.5. Observamos que a estimativa do parâmetros $\beta$ e $\lambda_{x}$ não é modificada fortemente pela suposição da razão de variâncias. Mas as variâncias assintóticas estimadas no modelo 1 e no modelo 2 para as estimativas de $\beta$

Ozán, N. S.

IME/USP 
é, respectivamente, 0.49 e 0.43 , o que indica que temos menor variância quando consideramos $\sigma_{e}^{2}=2 \sigma_{u}^{2}$. As variâncias assintóticas estimadas para as estimativas do parâmetro $\lambda_{x}$ para os modelos 1 é 5.78 e para o modelo 2 é 3.16. Portanto, consideramos o ajuste do modelo 2 como mas adequado. Ajustando um modelo de regressão usual o estimador de mínimos quadrados do parâmetro $\beta, \widehat{\beta}=\frac{S_{Y X}}{S_{X X}}$, é 0.87 , portanto estaríamos subestimando a estimação da taxa de mortalidade infantil para uma proporção de pobres dada, levando a conclusões erradas respeito a la taxa de mortalidade infantil.

Tabela 5.5 Estimativas de $\theta^{*}$

\begin{tabular}{lcccccc}
\hline \hline & $\widehat{\mu}_{x}$ & $\widehat{\beta}$ & $\widehat{\alpha}$ & $\widehat{\sigma}_{x}^{2}$ & $\widehat{\sigma}_{e}^{2}$ & $\widehat{\lambda}_{x}$ \\
\hline & & & & & & \\
Modelo 1 & 38.60 & 1.04 & 4.14 & 234.51 & 42.61 & 0.48 \\
Modelo 2 & 34.84 & 1.06 & 2.74 & 178.98 & 31.34 & 0.450 \\
\hline \hline
\end{tabular}

Tabela 5.6 Taxa de Mortalidade Infantil - Proporção de Pobres

\begin{tabular}{lcclcc}
\hline \hline Estado & $\begin{array}{c}\text { TMI } \\
(\mathrm{Y})\end{array}$ & $\begin{array}{c}\text { PP } \\
(\mathrm{X})\end{array}$ & Estado & $\begin{array}{c}\text { TMI } \\
(\mathrm{Y})\end{array}$ & $\begin{array}{c}\text { PP } \\
(\mathrm{X})\end{array}$ \\
\hline Rondônia & 33,06 & 16,31 & Sergipe & 45,45 & 39,97 \\
Acre & 44,18 & 30,80 & Bahia & 45,37 & 45,49 \\
Amazonas & 30,98 & 33,24 & Minas Gerais & 26,25 & 25,24 \\
Roraima & 38,26 & 16,98 & Espírito Santo & 17,68 & 22,59 \\
Pará & 34,63 & 33,64 & Rio de Janeiro & 21,28 & 11,76 \\
Amapá & 31,63 & 30,07 & São Paulo & 17,91 & 9,09 \\
Tocantins & 32,95 & 41,12 & Paraná & 19,67 & 20,62 \\
Maranhão & 54,16 & 57,39 & Santa Catarina & 16,35 & 14,21 \\
Piauí & 45,28 & 56,21 & Rio Grande do Sul & 15,10 & 16,55 \\
Ceará & 52,37 & 48,89 & Mato Grosso do Sul & 24,87 & 21,90 \\
Rio Grande do Norte & 48,66 & 41,03 & Mato Grosso & 27,45 & 21,06 \\
Paraíba & 60,25 & 44,48 & Goiás & 25,02 & 22,85 \\
Pernambuco & 58,17 & 40,94 & Distrito Federal & 22,64 & 15,28 \\
Alagoas & 66,13 & 51,28 & & & \\
\hline \hline
\end{tabular}

Ozán, N. S. 
Capítulo 6

\section{Conclusões}

\subsection{Considerações Finais}

Motivados pelo fato de que em diferentes situações aplicadas os dados não tem, necessariamente, uma distribuição simétrica, foi de nosso interesse aprofundar no estudo dos modelos com erros nas variáveis considerando distribuições mas gerais para os erros de modelo, que definimos como distribuições assimétricas. Definimos assim o modelo assimétrico com erros nas variáveis desde uma perspectiva geral. O modelo norma assimétrico com eros nas variáveis surge de considerar a distribuição normal assimétrica.

Existem várias formas de generalizar as distribuições elípticas para famílias mas gerais que as incluam. O interesse de assumir a proposta de Yuan e Bentler (1999a) é pelo fato de considerar uma estrutura para a matriz de covariância que capta a assimetria dos dados, além de ser uma generalização que modifica a assimetria e a curotse das distribuições elípticas. Este tipo de abordagem apresenta o inconveniente na hora de estimar os parâmetros do modelo usando o método de máxima verossimilhança. Portanto se origina assim o estudo do modelo normal assimétrico baseado na distribuição normal assimétrica (Azzalini, 19885).

Segundo a definição que consideramos para a distribuição normal assimétrica independente obtemos a expressão para a função de densidade do modelo normal assimétrico (Teorema 4.1), tendo assim uma importante generalização dos resultados existentes na literatura atual.

Destacamos os resultados assintóticos obtidos para o modelo assimétrico e o modelo normal assimétrico, onde observamos a influência da assimetria na variância assintótica dos estimadores do parâmetro $\beta$.

Os estimadores de máxima verossimilhança do modelo normal assimétrico são obtidos mediante métodos iterativos, analisando particularmente o estimador de $\beta$ mediante um estudo de simulação. Ressaltamos a aplicação num conjunto de dados relacionados com índices do desenvolvimento humano no Brasil.

Como resultados originais obtidos, destacamos, 
- a extensão do modelo de Yuan e Bentler (1999a) para a situação de modelos de regressão com erros nas variáveis, com a respectiva teoria assintótica para estimadores consistentes nos modelos aditivo e multiplicativo, obtendo resultados mais gerais que os propostos na literatura atual,

- a derivação da função de verossimilhança para o modelo normal assimétrico, com a correspondente teoria baseada nos resultados de máxima verossimilhança,

- o desenvolvimento da teoria assintótica para modelos de regressão com erros nas variáveis onde os erros tem distribuição normal assimétrica independente. Os resultados obtidos permitem extender o comportamento assintótico de alguns estimadores considerados sobre normalidade, além da obtenção de correções para ter consistência no modelo norml assimétrico,

- o analise da robustez do estimador do parâmetro $\beta$ mediante técnicas de simulação,

- o ajuste do caso particular de modelo normal assimétrico num conjunto de dados reais.

\subsection{Propostas de trabalho futuro}

O estudo do modelo assimétrico com erros nas variáveis oferece um importante desenvolvimento para futuras pesquisa. Em particular, fazemos as seguintes propostas:

- investigar a estimação dos parâmetros de assimetria e curtoseno modelo normal assimétrico com erros nas variáveis,

- desenvolver teste de Score para a assimetria do modelo normal assimétrico,

- derivar os resultados obtidos para outras formas de de definir distribuições assimétricas,

- abordar inferência nos modelos assimétricos usando metodologia Bayesiana.

Ozán, N. S. 


\section{Apêndice A}

\section{A.1 Método de Max-BFGS}

Para determinar o máximo da função de log-verossimilhança no Capítulo 5, utilizamos a função MaxBFGS do linguagem de programação $O x$. Esta rotina maximiza uma função usando o método de quase- Newton desenvolvido por Broyden, Fletcher e Shanno (Dornik, 1996). Maximiza-se a função $f(\theta)$ considerando o valor do máximo na iteração $k+1$ como,

$$
\theta(k+1)=\theta(k)+s(k) \mathrm{Q}^{-1}(k) q(k),
$$

onde, $\theta(k)$ é o máximo da função na iteração $k, s(k)$ é tamanho do passo, que usualmente é $1, \mathrm{Q}(k)$ matriz simétrica positiva definida e $q(k)$ primeira derivada da função a maximizar (vetor escore).

O método pode usar primeira derivada analítica ou numérica e o processo de iteração não é influenciado pela escolha destas derivadas. Também, Max-BFGS, avalia e atualiza diretamente $\mathrm{H}=\mathrm{Q}^{-1}$ ( $\mathrm{H}$, matriz hessiana). Neste caso, a diferença com o método de Newton, a matriz hessiana é aproximada por uma matriz simétrica positiva definida, que é atualizada para cada iteração.

Dois critérios de convergência são possíveis, um deles baseado no vetor escore $q(k)$, nulo quando é avaliado no máximo, e outro na diferencia $\delta(k)=q(k)-q(k-1)$ que deve ser pequena, ou também podemos considerar $\delta(k)=\theta(k)-\theta(k-1)$.

É importante destacar que a matiz quase-hessiana não pode ser usada para determinar os desvios padrões. Portanto, para obter a matriz de informação observada usamos a subrotina Num2Derivate, que fornece os valores da segunda derivada em relação as estimativas dos parâmetros.

\section{A.2 Programas de maximização}

A seguir, apresentamos os programas utilizados no Capítulo 5 para o Estudo de Simulação e a Aplicação. O programa para o primeiro dos estudos é o básico para maximizar.

\section{A.2.1 Programa para o Estudo 1}

\#include <oxstd.h>

\#include <oxfloat.h> //define $M_{-} P_{1}$ e $M_{-} P_{2}, \pi$ e $2 \pi$, respectivamente 
\#include $<$ maximize.h $>$

\#pragma link("maximize.oxo")

static decl g_dados;

static decl $g_{-} n ; / /$ tamanho da amostra

// Função a ser maximizada dividida por $n$

fLoglik(const vP, const adFunc, const avScore, const amHess)

\{

decl mu, sigma, $m u_{-} x$, beta, alpha, sigma2x, sigma2e,

sigma2u, lambda, parcela1, d, parcela2, ci, cj, dfunc, vr;

$/ / m u_{-} x=\mathrm{vP}[0]$;

$/ /$ beta $=v P[1]$

$/ /$ alpha $=\mathrm{vP}[2]$

$/ /$ sigma2x $=\mathrm{vP}[3] ;$

$/ /$ sigma2e $=v P[4] ;$

$/ /$ lambda $=v P[5] ;$

$/ / \operatorname{sigma2u}=$ sigma2e $=v P[4]:$

// vetor de medias mu

$\mathrm{mu}=\operatorname{zeros}(2,1)$

$\mathrm{mu}[0][0]=\mathrm{vP}[2]+\mathrm{vP}[1] * \mathrm{vP}[0]$;

$\mathrm{mu}[1][0]=\mathrm{vP}[0]$;

// matriz de covariâncias sigma

$\operatorname{sigma} 2 \mathrm{u}=\mathrm{vP}[4]$;

$\operatorname{sigma}=\operatorname{zeros}(2,2) ;$

sigma $[0][0]=\left(v P[1]^{2}\right) * v P[3]+v P[4] ;$

sigma $[0][1]=\mathrm{vP}[1]{ }^{*} \mathrm{vP}[3] ;$

sigma $[1][0]=\operatorname{sigma}[0][1]$;

$\operatorname{sigma}[1][1]=\mathrm{vP}[3]+\mathrm{vP}[4] ;$

// calculo da parcela1

parcela1 $=\operatorname{zeros}\left(g_{-} n, 1\right)$;

for $\left(\mathrm{ci}=0 ; \operatorname{ci} ; g_{-} n ;++\mathrm{ci}\right)$

\{

parcela1 $[\mathrm{ci}][0]=\left(g_{-} \operatorname{dados}[][\mathrm{ci}]-\mathrm{mu}\right)^{2} *_{\text {invert }}(\operatorname{sigma}) *\left(g_{-} \operatorname{dados}[][\mathrm{ci}]-\mathrm{mu}\right)$;

\}

// calculo de $\mathrm{d}$

$\left.\mathrm{d}=v P[5] * v P[3] *\left(v P[3]+v P[5]^{(2)} /\left(1 / v P[3]+v P[1]^{2} / v P[4]+1 / v P[4]\right)\right)^{(}-0.5\right) ;$

$/ /$ calculo da parcela2

parcela $2=\operatorname{zeros}\left(g_{-} n, 1\right)$;

Ozán, N. S.

IME/USP 


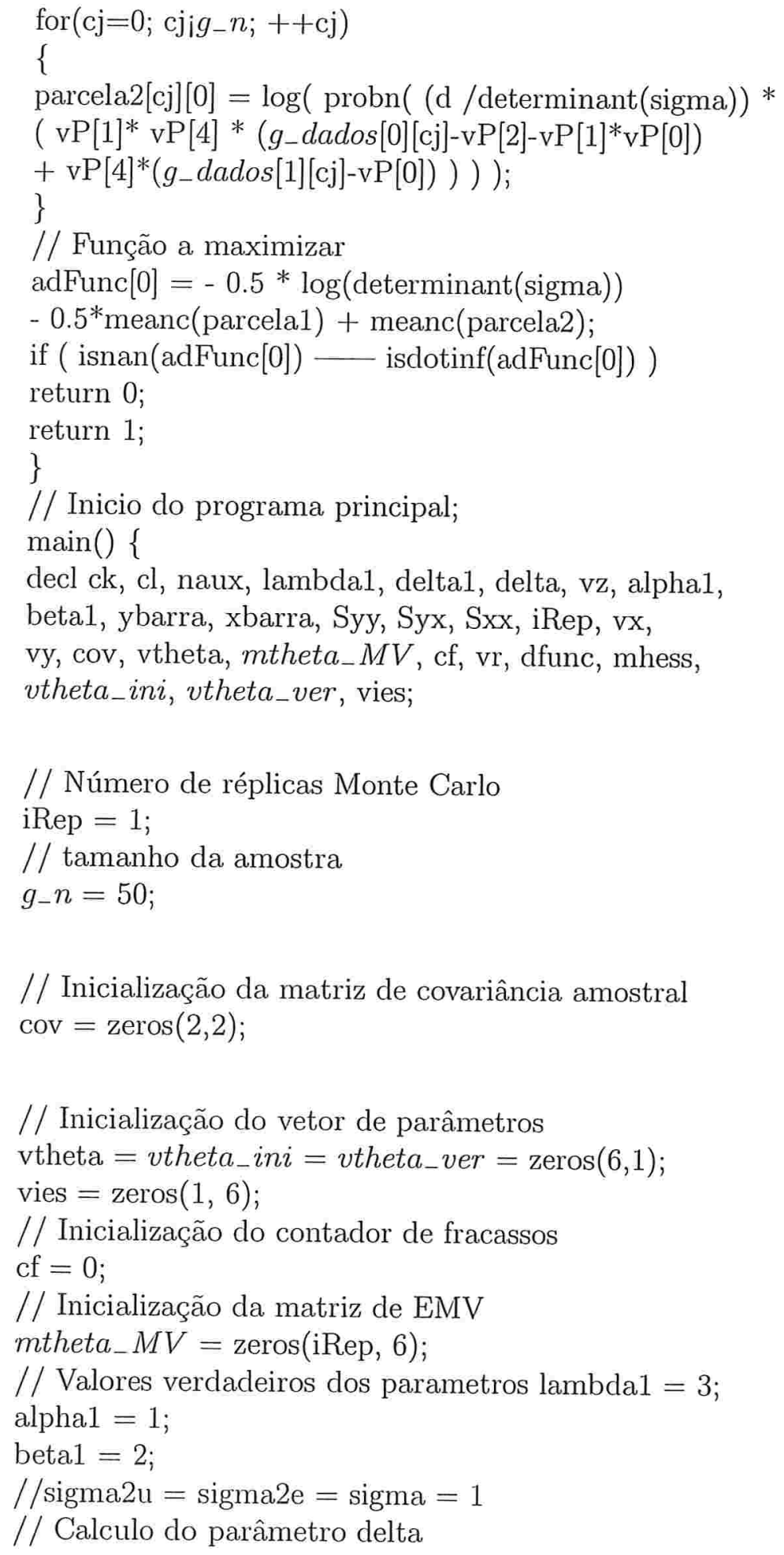

Ozán, N. S. 
delta $1=$ lambda1 /sqr
$/ /$ Valores verdadeiros
vtheta_ver $[0][0]=1 ;$
vtheta_ver $[1][0]=2 ;$
vtheta_ver $[2][0]=1 ;$
vtheta_ver $[3][0]=1 ;$
vtheta_ver $[4][0]=1 ;$
vtheta_ver $[5][0]=3 ;$

// Definição do gerador de números aleatorios ranseed( "GM");

// Loop de Monte Carlo for $\left(\mathrm{ck}=0\right.$; $\left.\mathrm{ck}_{j} \mathrm{iRep}+\mathrm{cf} ;++\mathrm{ck}\right)$

\{

// Geração dos dados

ranseed $(\mathrm{ck}+11,3 * \mathrm{ck}) ; / /$ semente do gerador

$\mathrm{vz}=\operatorname{delta1}{ }^{*}$ fabs $\left(\operatorname{rann}\left(g_{-} n, 1\right)\right)+\operatorname{sqrt}\left(1-\operatorname{delta} 1^{2}\right) * \operatorname{rann}\left(g_{-} n, 1\right) ;$

$\mathrm{vy}=\operatorname{alpha} 1+\operatorname{beta1}{ }^{*}(\mathrm{vz}+1)+\operatorname{rann}\left(g_{-} n, 1\right) ;$

$\mathrm{vx}=(\mathrm{vz}+1)+\operatorname{rann}\left(g_{-} n, 1\right) ;$

$g_{-}$dados $=\left(\begin{array}{ll}\mathrm{vy} & \mathrm{vx}\end{array}\right)^{\prime}$;

// Matriz de covariâncias amostral

cov $=\operatorname{variance}\left(g_{-}\right.$dados $\left.{ }^{\prime}\right)$;

Syy $=\operatorname{cov}[0][0] ;$

Syx $=\operatorname{cov}[0][1]$

$\operatorname{Sxx}=\operatorname{cov}[1][1]$

// Medias amostrais

ybarra $=\operatorname{meanc}(v y)$;

xbarra $=\operatorname{meanc}(\mathrm{vx})$;

// Valores iniciais dos parametros (MM)

vtheta $[0][0]=$ xbarra;

vtheta $[1][0]=\left(\right.$ Syy $\left.-\operatorname{Sxx}+\operatorname{sqrt}\left((S y y-S x x)^{2}+4^{*} S y x^{2}\right)\right) /(2 * \operatorname{Syx})$;

vtheta[2][0] = ybarra - vtheta[1]*xbarra;

vtheta[3][0] = Syx $/$ vtheta[1];

vtheta $[4][0]=$ Syy - vtheta $[1]^{*}$ Sxx;

vtheta $[5][0]=\left(\left(\operatorname{meanc}\left((v x-x b a r r a) .^{3}\right)^{2}\right) /\left(\operatorname{meanc}\left((v x-x b a r r a) .^{2}\right)\right)^{3}\right)$;

Ozán, N. S.

IME/USP 
vtheta_ini $=$ vtheta;

// Maximizacao da log-verossimilhança:

$\mathrm{vr}=$ MaxBFGS(fLoglik, \&vtheta, \&dfunc, 0, TRUE);

//Matriz de Informação observada

mhess $=\operatorname{unit}(6)$;

Num2Derivative(fLoglik, vtheta, \&mhess);

// Checa as estimativas das variancias

if $($ vtheta $[3][0]<0 \|$ vtheta $[4][0]<0$

||vtheta[5][0]<-5\|vtheta[5][0] $>5)$

$++\mathrm{cf}$;

if $($ vtheta $[3][0]<0 \|$ vtheta[4][0] $<0$

||vtheta[5][0]<-5||vtheta[5][0]>5)

continue;

// Checa a convergencia e guarda o resultado:

if $\left(\mathrm{vr}==M A X_{-} C O N V \| v r==M A X_{-} W E A K_{-} C O N V\right)$

\{

mtheta_ $M V$ [ck-cf] []$=$ vtheta';

\}

else

\{

++cf; // replica de MC nao convergiu

\}

delta $=$ vtheta $[5] /\left(1-\text { vtheta }[5]^{2}\right)^{2} ;$

\}

// Fim do loop de Monte Carlo

\section{A.2.2 Programa para o Estudo 3}

\#include <oxstd.h>

\#include <oxfloat.h $>/ /$ define $M_{-} P_{1}$ e $M_{-} P_{2}, \pi$ e $2 \pi$, respectivamente

\#include $<$ maximize.h $>$

\#pragma link("maximize.oxo")

static decl g_dados;

static decl $g_{-} n ; / /$ tamanho da amostra 







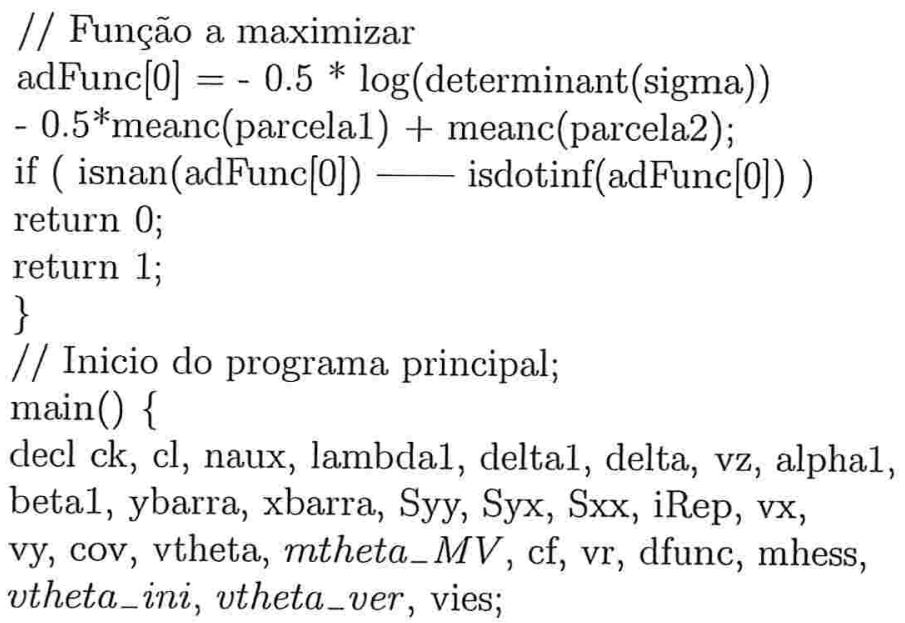

// Número de réplicas Monte Carlo

iRep $=10 ; / /$ Este valor varia com $\mathrm{k}$ réplicas.

// tamanho da amostra

$g_{-} n=50$

// Inicialização da matriz de covariância amostral $\operatorname{cov}=\operatorname{zeros}(2,2)$;

// Inicialização do vetor de parâmetros

vtheta $=$ vtheta_ini $=$ vtheta_ver $=\operatorname{zeros}(6,1)$;

vies $=\operatorname{zeros}(1,6)$;

// Inicialização do contador de fracassos

$\mathrm{cf}=0$;

// Inicialização da matriz de EMV

mthet $a_{-} M V=$ zeros(iRep, 6);

// Valores verdadeiros dos parametros lambda $1=3$;

alpha1 $=1$;

beta1 $=2$;

$/ / \operatorname{sigma} 2 \mathrm{u}=\operatorname{sigma} 2 \mathrm{e}=\operatorname{sigma}=1$

// Calculo do parâmetro delta

delta $1=$ lambda $1 / \operatorname{sqrt}\left(1+l a m b d a 1^{2}\right)$;

// Valores verdadeiros

vtheta_ver $[0][0]=1$;

vtheta_ver $[1][0]=2$;

vtheta_ver $[2][0]=1$;

vtheta_ver $[3][0]=1$;

Ozán, N. S. 
vtheta_ver $[4][0]=1$;

vtheta_ver $[5][0]=3$;

// Definição do gerador de números aleatorios ranseed( "GM");



// Matriz de covariâncias amostral cov $=\operatorname{variance}\left(g_{-}\right.$dados $\left.{ }^{\prime}\right)$;

Syy $=\operatorname{cov}[0][0] ;$

Syx $=\operatorname{cov}[0][1] ;$

$\operatorname{Sxx}=\operatorname{cov}[1][1]$

// Medias amostrais

ybarra $=$ meanc $(v y)$;

xbarra $=\operatorname{meanc}(v x)$;

// Valores iniciais dos parametros (MM)

vtheta $[0][0]=$ xbarra;

vtheta $[1][0]=\left(\right.$ Syy $\left.-\operatorname{Sxx}+\operatorname{sqrt}\left((S y y-S x x)^{2}+4^{*} S y x^{2}\right)\right) /\left(2^{*} \operatorname{Syx}\right)$

vtheta[2][0] = ybarra - vtheta[1]*xbarra;

vtheta[3][0] = Syx/vtheta[1];

vtheta $[4][0]=$ Syy - vtheta $[1] *$ Sxx;

$\left.\operatorname{vtheta}[5][0]=\left(\left(\operatorname{meanc}((v x-x b a r r a))^{3}\right)^{2}\right) /\left(\operatorname{meanc}\left((v x-x b a r r a) \cdot{ }^{2}\right)\right)^{3}\right)$;

vtheta_ini $=$ vtheta;

// Maximizacao da log-verossimilhança:

vr = MaxBFGS(fLoglik, \&vtheta, \&dfunc, 0, TRUE);

//Matriz de Informação observada

Ozán, N. S. 
mhess $=\operatorname{unit}(6)$;

Num2Derivative(fLoglik, vtheta, \&mhess);

// Checa as estimativas das variancias

if $($ vtheta $[3][0]<0 \|$ vtheta $[4][0]<0$

$\|$ theta $[5][0]<-5 \|$ vtheta $[5][0]>5)$

$++\mathrm{cf} ;$

if $($ vtheta $[3][0]<0 \|$ vtheta $[4][0]<0$

||vtheta[5][0]<-5||vtheta[5][0] > 5)

continue;

// Checa a convergencia e guarda o resultado:

if $\left(\mathrm{vr}==M A X_{-} C O N V \| v r==M A X_{-} W E A K_{-} C O N V\right)$

\{

mtheta $a_{-} M V[$ ck-cf $][]=$ vtheta';

\}

else

\{

$++\mathrm{cf} ; / /$ replica de MC nao convergiu

\}

delta $=$ vtheta $[5] /\left(1-\text { vtheta }[5]^{2}\right)^{2} ;$

\}

// Fim do loop de Monte Carlo

print $(" \backslash n \backslash n$ Estimativas iniciais = ", vtheta_ini);

print(" $\backslash n \backslash n$ Media das estimativas = ", meanc $\left(\right.$ mtheta_$\left._{-} M V\right)$ );

print(" $\backslash n \backslash n$ Variancia das estimativas $=", \operatorname{varc}($ mtheta_$M V))$;

vies $=$ meanc $($ mtheta_ $M V)-$ vtheta_ver';

$\operatorname{print}(" \backslash n \backslash n$ Vies da media das estimativas = ", vies);

print(" $\backslash n \backslash n$ EQM das estimativas = ", $\operatorname{varc}($ mtheta $M V)+\operatorname{sqr}(\operatorname{vies}))$;

print(" $\backslash n \backslash n$ Matriz de estimativas = ", mtheta_MV);

print(" $\backslash n \backslash n$ Numero de replicas MC = ", iRep);

print(" $\backslash n \backslash n$ Numero de fracassos = ", cf);

$\operatorname{print}\left(" \backslash n \backslash n\right.$ Tamanho da amostra $=$ ", $\left.g_{-} n\right)$;

print(" $\backslash n \backslash n$ Valor Real Lambda = ", lambda1);

print(" $\backslash n \backslash n$ Valor delta $=$ ", delta);

print(" $\backslash n \backslash n$ Matriz de informação observada = ", $(-1)^{*}$ mhess);

print(" $\backslash n \backslash n$ Matriz de covariancia observada $="$,

$(-1)$ *invert(mhess));

Ozán, N. S. 
\}

/* Fim do programa */

\section{A.2.3 Programa para a Aplicação}

\#include <oxstd.h $>$

\#include <oxfloat.h $>/ /$ define $M_{-} P_{1}$ e $M_{-} P_{2}, \pi$ e $2 \pi$, respectivamente

\#include $<$ maximize.h $>$

\#pragma link("maximize.oxo")

static decl $g_{-}$dados;

static decl $g_{-} n$; // tamanho da amostra

// Função a ser maximizada dividida por $\mathrm{n}$

fLoglik(const vP, const adFunc, const avScore, const amHess)

\{

decl mu, sigma, $m u_{-} x$, beta, alpha, sigma2x, sigma2e,

sigma2u, lambda, parcela1, d, parcela2, ci, cj, dfunc, vr;

$/ / m u_{-} x=\mathrm{vP}[0]$;

$/ /$ beta $=v P[1]$

$/ /$ alpha $=\mathrm{vP}[2] ;$

$/ / \operatorname{sigma} 2 \mathrm{x}=\mathrm{vP}[3]$

$/ /$ sigma2e $=\mathrm{vP}[4]$

// lambda $=v P[5]$

$/ / \operatorname{sigma} 2 \mathrm{u}=\operatorname{sigma} 2 \mathrm{e}=\mathrm{vP}[4]:$

// vetor de medias mu

$\mathrm{mu}=\operatorname{zeros}(2,1)$

$\mathrm{mu}[0][0]=\mathrm{vP}[2]+\mathrm{vP}[1] * \mathrm{vP}[0]$

$\mathrm{mu}[1][0]=\mathrm{vP}[0]$;

// matriz de covariâncias sigma

sigma $2 \mathrm{u}=\mathrm{vP}[4]$;

$\operatorname{sigma}=\operatorname{zeros}(2,2) ;$

sigma $[0][0]=\left(v P[1]^{2}\right) * v P[3]+v P[4]$;

sigma $[0][1]=\mathrm{vP}[1]{ }^{*} \mathrm{vP}[3]$;

sigma $[1][0]=\operatorname{sigma}[0][1]$;

$\operatorname{sigma}[1][1]=v P[3]+v P[4] ;$

// calculo da parcela1

parcela1 $=\operatorname{zeros}\left(g_{-} n, 1\right)$;

for $\left(\mathrm{ci}=0 ; \operatorname{ci} \mathrm{i} g_{-} n ;++\mathrm{ci}\right)$

Ozán, N. S. 


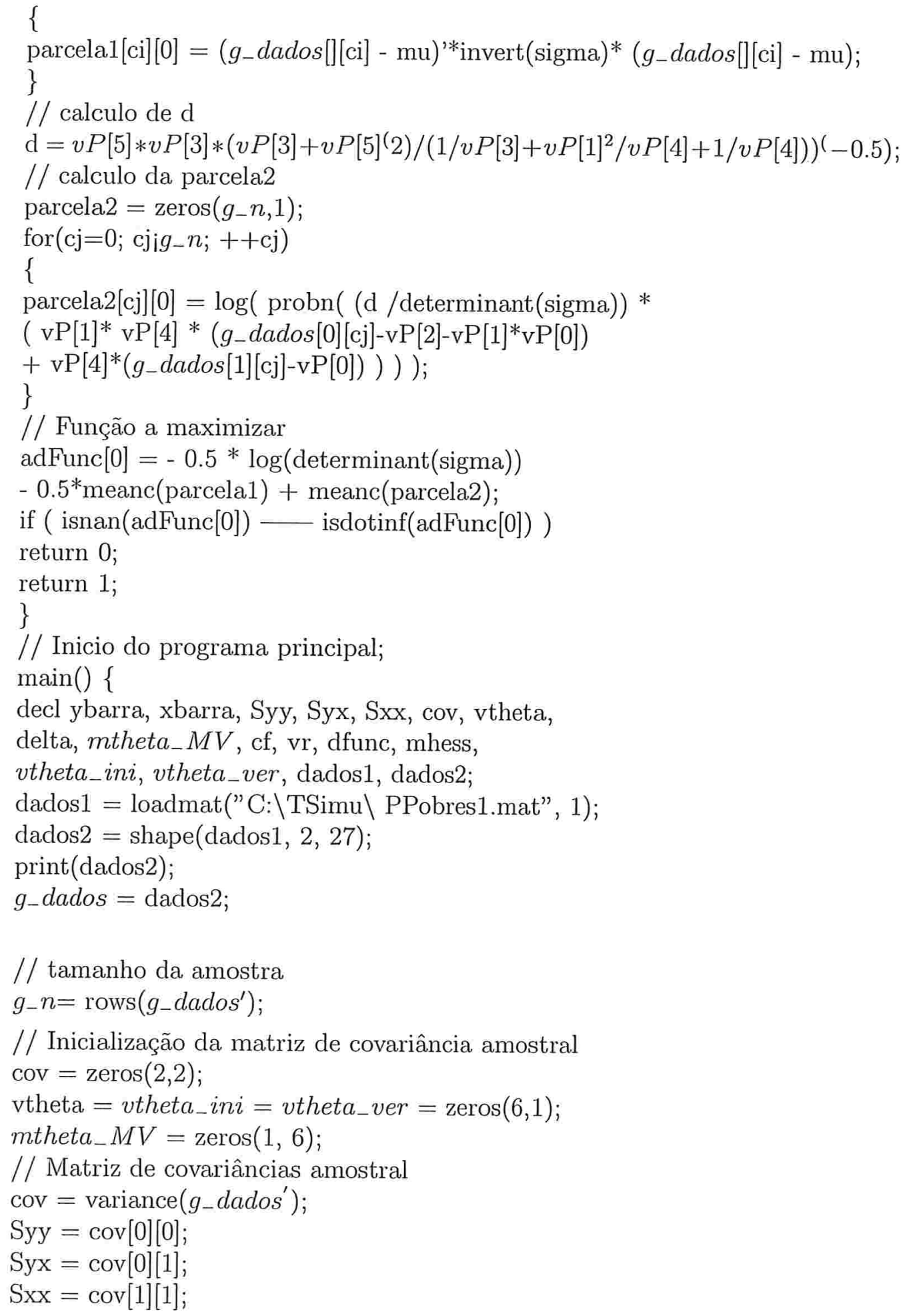

Ozán, N. S. 


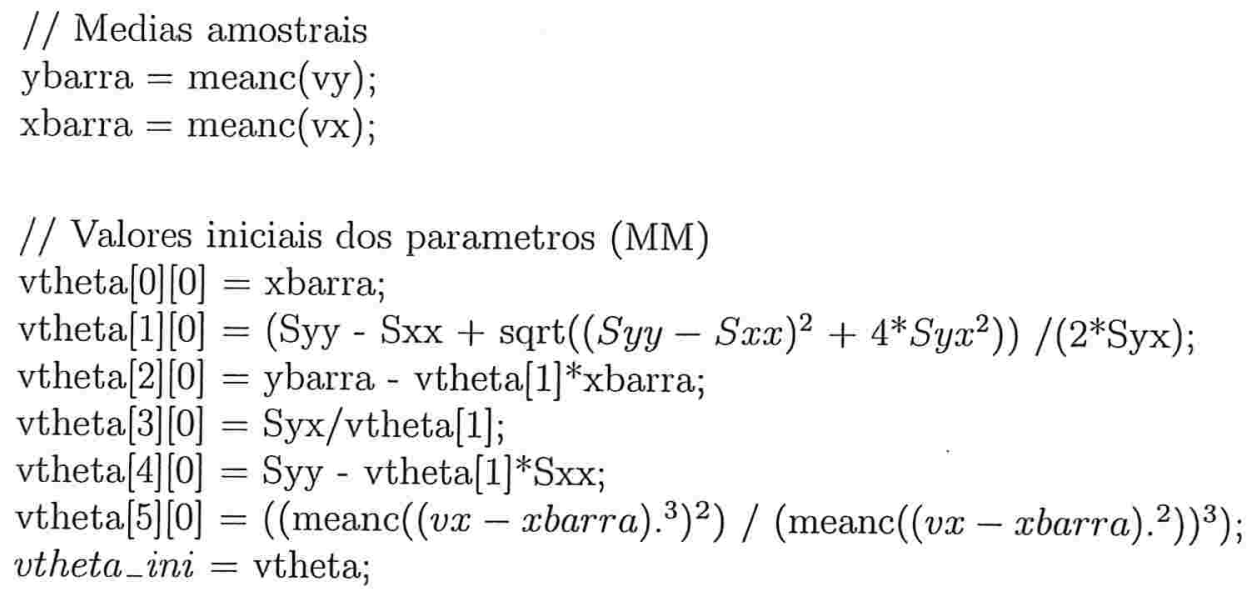

// Maximizacao da log-verossimilhança:

vr = MaxBFGS(fLoglik, \&vtheta, \&dfunc, 0, TRUE);

//Matriz de Informação observada

mhess $=\operatorname{unit}(6)$;

Num2Derivative(fLoglik, vtheta, \&mhess);

// Checa a convergencia e guarda o resultado:

if(vr $\left.==M A X_{-} C O N V \| v r==M A X_{-} W E A K_{-} C O N V\right)$

\{

mtheta_MV [ck-cf] []$=$ vtheta';

\}

else

\{

$++c f ; / /$ replica de $\mathrm{MC}$ nao convergiu

\}

delta $=$ vtheta $[5] /\left(1-\text { vtheta }[5]^{2}\right)^{2} ;$

\}

print(" $\backslash n \backslash n$ Estimativas iniciais = ", vtheta_ini);

print(" $\backslash n \backslash n$ Matriz de estimativas =", mtheta_MV);

print(" $\backslash n \backslash n$ Tamanho da amostra $=$ ", $\left.g_{-} n\right)$;

print(" $\backslash n \backslash n$ Matriz de informação observada = ", $(-1)^{*}$ mhess);

print(" $\backslash n \backslash n$ Matriz de covariancia observada = ",

$(-1) *$ invert(mhess));

\}

/* Fim do programa */

Ozán, N. S. 


\section{Referências Bibliográficas}

[1] Arellano-Valle, R. and Bolfarine, H. (1996). Elliptical structural model. Communication in in Statististics. Theory and Methodos, 25, 10, 2319-2342.

[2] Arellano-Valle, R., Bolfarine, H. and Gasco, L. (2002). Meassurement Error Models with Nonconstant Covariance Matrices. Journal of Multivariate Analysis, 82, 395-415.

[3] Arellano-Valle, R., Bolfarine, H. and Vilca-Labra, F. (1996). Ultraestructural eliptical model. The Canadian Journal of Statistics, 24, 207-216.

[4] Arellano-Valle, R.B., del Pino, G. and San Martin, E. (2002). Definition and probabilistic properties of skew-distributions. Statistics and Probability Letters, 58, 111121.

[5] Azzalini, A. (1985). A class of distributions which includes the normal ones. Scandinavian Journal of Statististics, 12, 171-178.

[6] Azzalini, A. and Capitanio, A. (1999). Statistical Applications of the Multivariate Skew Normal Distributions. Journal Royal Statistic Society, B, 61, 579-602.

[7] Azzalini, A. and Dalla-Valle, A. (1996). The multivariate skew-normal distribution. Biometrika, 83, 715-726.

[8] Bolfarine, H. and Arellano-Valle, R. (1999). Weak nondifferential measurement error models. Statistics and Probability Letters, 40, 279-287.

[9] Bolfarine, H. and Cordani L. (1993). Estimation of Structural linear regress model with a known reliability ratio. Anals of the Institute of Statistical Mathematics, 45, $3,531-540$.

[10] Branco, M. and Dey D. (2001). A general class of multivariate skew-eliptical distribution. Journal of Multivariate Analysis (to appear).

[11] Chen, C. e Van Ness, J. (1991). On the unreplicate ultrastructural model. Biometrika, $44,2-445$.

[12] Dolby, G.R. (1976). A ultrastructural relation: A synthesis of the functional and structural relations. Biometrika, 63, 39-50. 
[13] Fang, K.T., Kotz, S.e Ng, K.W. (1990). Symmetrical multivariate and related distribution. Chapman and Hall. London.

[14] Fuller, W. (1987). Measurement error models. Wiley, New York.

[15] Gleser, L.J. (1981). Estimate in a multivariate errors in variables regression model large sample results. Annals of Statistics, 9, 24-44.

[16] Gleser, L.J. (1985). A note on G.R. Dolby's unreplicate ultraestructural error in variables model. Biometrika, 72, 117-129.

[17] Kendall, M. e Stuart (1979). The advanced theory of Statistics. Vol 2, Griffin, London.

[18] Lindley, D.V. and El-Sayad, G. (1968). The Bayesian estimation of a linear functional relationship. Journal of the Royal Statistical Society, B,30, 190-202.

[19] Magnus, J. e Neudecker, H. (1979). The commutation matrix,some properties and applications. Annals of Statistics, 7, 381-395.

[20] Magnus, J. e Neudecker, H. (1988). Matrix differential calculus with applications in statistics and econometrics. Wiley, N.Y.

[21] Mardia, K. (1970). Measures of multivariate skewness and kurtosis with applications. Biometrika, 57, 519-530.

[22] Muirhead, R. (1982). Aspects of multivariate statistical theory. Wiley, New York.

[23] Yuan, K. e Bentler, P. (1999). On normal theory and associate test statistics in covaariance Structure analysis under two classes of nonormal distributions. Statistics Sinica 9, 831-853.

[24] Yuan, K. e Bentler, P. (2000). Inferences in Correlation in some Classes of Nonormal Distributions. Journal of Multivariate Analysis, 72, 230-248. 